\title{
Definitionen und Anerkennung substaatlicher Gruppen im Völkerrecht
}

\section{Philipp Socha}

Göttinger Studien zu Cultural Property, Band 12 

Philipp Socha

Definitionen und Anerkennung substaatlicher Gruppen im Völkerrecht

Dieses Werk ist lizenziert unter einer

Creative Commons

Namensnennung - Weitergabe unter gleichen Bedingungen

4.0 International Lizenz.

(c). (i) (2) 
erschienen als Band 12 in der Reihe „Göttinger Studien zu Cultural Property“ im Universitätsverlag Göttingen 2017 
Philipp Socha

Definitionen

und Anerkennung

substaatlicher Gruppen

im Völkerrecht

Eine Untersuchung

der rechtlichen Anwendung

völkerrechtlicher Konstruktionen

substaatlicher kollektiver Identitäten

und aktueller Entwicklungen im

Intergovernmental Committee der WIPO

Göttinger Studien

zu Cultural Property, Band 12

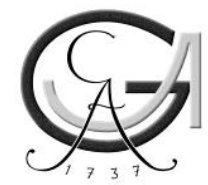

Universitätsverlag Göttingen 2017 


\title{
Bibliographische Information der Deutschen Nationalbibliothek
}

Die Deutsche Nationalbibliothek verzeichnet diese Publikation in der Deutschen Nationalbibliographie; detaillierte bibliographische Daten sind im Internet über $<$ http://dnb.dnb.de $>$ abrufbar.

Gedruckt mit Hilfe der Deutschen Forschungsgemeinschaft (DFG)

\author{
Autorenkontakt \\ Philipp Socha \\ E-Mail: philippsocha@gmail.com
}

Dieses Buch ist auch als freie Onlineversion über die Homepage des Verlags sowie über den Göttinger Universitätskatalog (GUK) bei der Niedersächsischen Staats- und Universitätsbibliothek Göttingen (http://www.sub.uni-goettingen.de) erreichbar. Es gelten die Lizenzbestimmungen der Onlineversion.

Lektorat und Satz: Philipp Socha

Umschlaggestaltung: Margo Bargheer, Stefan Groth

(C) 2017 Universitätsverlag Göttingen

http://univerlag.uni-goettingen.de

ISBN: 978-3-86395-269-3

eISSN: 2512-6342 
„Göttinger Studien zu Cultural Property“ / "Göttingen Studies in Cultural Property”

Reihenherausgeber

Regina Bendix

Kilian Bizer

Brigitta Hauser-Schäublin

Gerald Spindler

Peter-Tobias Stoll

Editorial Board

Andreas Busch, Göttingen

Rosemary Coombe, Toronto

Ejan Mackaay, Montreal

Dorothy Noyes, Columbus

Achim Spiller, Göttingen

Bernhard Tschofen, Zürich

Homepage

http://gscp.cultural-property.org 

Meinen Eltern 



\section{Vorwort}

Die Leistung zum Abschluss einer Dissertation besteht nur zum Teil aus der wissenschaftlichen Denkarbeit. Mindestens genauso wichtig sind Durchhaltevermögen sowie ein Mindestmaß an Unterstützung durch Institutionen und vor allem durch Familie, Freunde und Kollegen. Daher möchte ich diese Zeilen nutzen, um den besonderen Menschen, ohne die diese Arbeit nicht möglich gewesen wäre, meine Anerkennung und meinen Dank auszusprechen.

Dies ist zuerst meine Schwester, die mir in zahlreichen Telefonaten ein interdisziplinärer Spiegel und intellektuell unermüdlicher, wie auch geduldiger Diskussionspartner war, und mit der ich die theoretischen und politischen Konsequenzen meiner Argumente ergründen konnte. Gleichzeitig gaben sie und der Rest meiner Familie sowie meine Freunde Anja Eikermann, Marius Stankoweit, Jonas Bermaoui und Rachel Harris mir die emotionale Unterstützung, die für so ein Projekt notwendig ist. Lena Schnieder darf ich sehr für das finale Korrekturlesen der Arbeit vor Drucklegung danken.

Natürlich wäre diese Arbeit nie ohne meinen Doktorvater Peter-Tobias Stoll zustande gekommen, dem ich für die Betreuung meines Projekts und auch für die Begleitung über das Wissenschaftliche hinaus danken möchte. Er trägt zusammen mit Andreas Paulus, dem ich hier für die Erstellung des Zweitgutachtens danken möchte, den völkerrechtlichen Teil des Instituts für Völkerrecht und Europarecht der Georg-August-Universität Göttingen. Das Institut mit seinen Mitarbeitern, Studenten, Doktoranden und Professoren sowie der Bibliothek war für mich lange Jahre wie ein zu Hause und damit auch die Umgebung, die diese Arbeit ermöglicht hat.

Institutionell ebenso wichtig war der Rahmen der DFG-Forschergruppe Cultural Property unter der Leitung von Regina Bendix. Die interdisziplinäre Zusammenarbeit, die sich insbesondere für den Beitrag zusammen mit Karin Klenke ergab, war eine der notwendigen geistigen Quellen meiner Arbeit. Und schließlich darf ich für das Stipendium des Cusanuswerks danken, welches mir die notwendige finanzielle Unabhängigkeit gesichert hat.

Berlin, Februar 2017 



\section{Inhaltsübersicht}

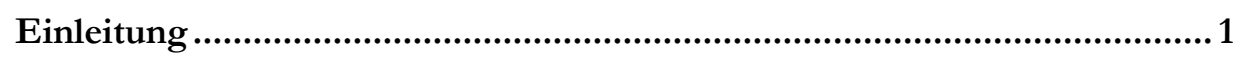

A. Substaatliche Gruppen im Völkerrecht ……….............................................

B. Grundlagen und Ziel der Untersuchung............................................................6

Teil 1: Substaatliche Gruppen im Völkerrecht ............................................ 13

A. Der internationale Minderheitenschutz ……………………………………......13

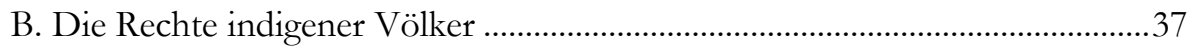

C. Lokale Gemeinschaften.....................................................................................59

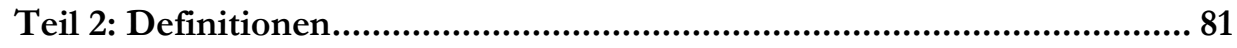

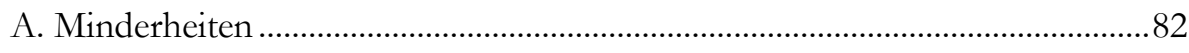

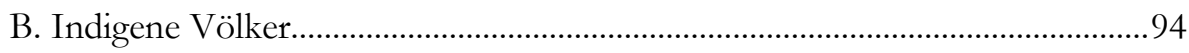

C. Lokale Gemeinschaften...................................................................................102

D. Selbstidentifikation kultureller Distinktion als Kern der

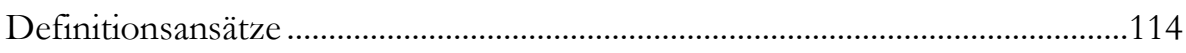

E. Das rechtliche Verhältnis der Konzepte zueinander ......................................116

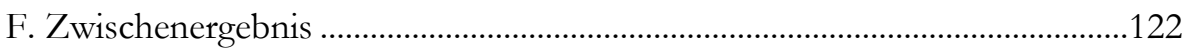

Teil 3: Anerkennung ..............................................................................125

A. Anerkennungsverfahren: eine Auswahl ..........................................................126

B. Rechtliche Bewertung der Anerkennung substaatlicher Gruppen ...............150

C. Interdisziplinäre Perspektiven: Konstruktivismus und Anerkennung..........163

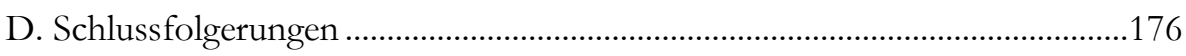

Teil 4: Verhandlungen im WIPO Intergovernmental Committee ..............181

A. Geschichte und Hintergrund........................................................................182

B. Die beneficiaries neuer Instrumente zum Schutz von TK/TCEs.................211

C. Die Anerkennung von Berechtigten: die NCA und neue

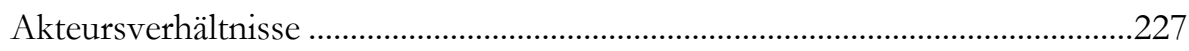

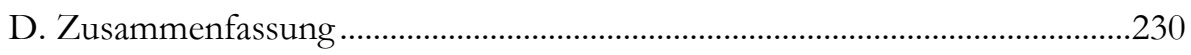

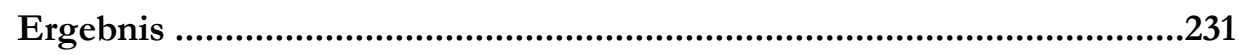

Zusammenfassung in Thesen ............................................................... 235 
A. Primärquellen: Verträge, Gesetzgebung, Sitzungsunterlagen,

Dokumente, Berichte, Entscheidungen, Nachrichten ........................................ 241

B. Sekundärquellen: Monographien, Artikel, Beiträge in Sammelwerken ....... 256 


\section{Inhaltsverzeichnis}

Einleitung ...........................................................................................

A. Substaatliche Gruppen im Völkerrecht ........................................................

I. Die bestehenden Konzepte substaatlicher Gruppen ......................................

II. Das Problem einer rechtlichen Definition von kulturell distinkten

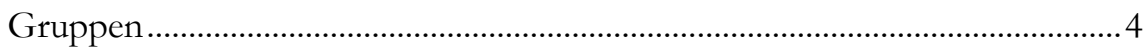

B. Grundlagen und Ziel der Untersuchung..........................................................6

I. Gegenstand und Forschungsfrage ………………………………………....6

II. Gang der Untersuchung............................................................................. 6

1. Konzepte substaatlicher Gruppen.............................................................

2. Definitionsansätze substaatlicher Gruppen ................................................ 8

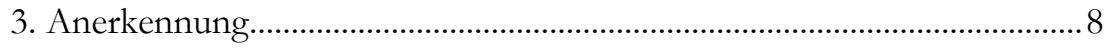

4. Die Verhandlungen im IGC der WIPO ..................................................

III. Ziel der Untersuchung ....................................................................................10

Teil 1: Substaatliche Gruppen im Völkerrecht ......................................... 13

A. Der internationale Minderheitenschutz ……………………………………....13

I. Geschichte des Minderheitenschutzes im Völkerrecht ..................................14

II. Rechtsquellen des internationalen Minderheitenschutzes ............................16

1. Historische Rechtsquellen..........................................................................17

2. Rechtsquellen aus der Zeit des Völkerbundes...........................................17

3. Minderheitenschutzinstrumente im modernen Völkerrecht....................18

4. Neuere Entwicklungen im Sinne einer kollektivrechtlichen

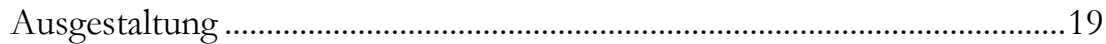

III. Inhalt des internationalen Minderheitenschutzes........................................21

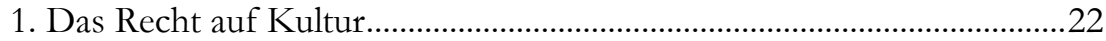

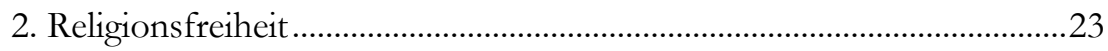

3. Das Recht zum Gebrauch der eigenen Sprache .......................................24 
4. Umsetzung des internationalen Minderheitenschutzes ............................25

a) Eingriffsverbot.......................................................................................26

b) Schutzpflicht des Staates bei Handlungen Privater .............................27

c) Aktive Schutz- und Unterstützungsmaßnahmen ................................28

IV. Berichts- und Beschwerdeverfahren des internationalen

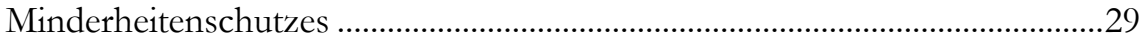

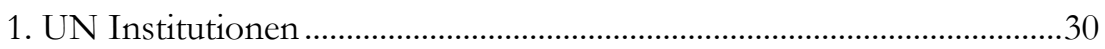

a) Historische Struktur der UN Institutionen

des Minderheitenschutzes ..........................................................................31

b) Forum on Minority Issues .....................................................................

c) Independent Expert on Minority Issues................................................32

d) Human Rights Council.........................................................................32

e) Complaint Procedure im Human Rights Council .................................32

2. Berichts- und Beschwerdemechanismen unter dem IPbpR ......................33

3. Berichtsverfahren der Framework Convention............................................34

4. Der European High Commissioner on National Minorities

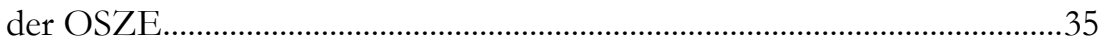

5. Durchsetzung bilateraler Verträge ................................................................35

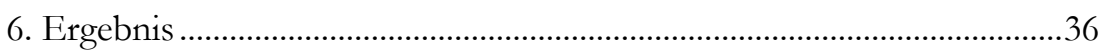

V. Zusammenfassung: ältestes Konzept zum Schutz kulturell

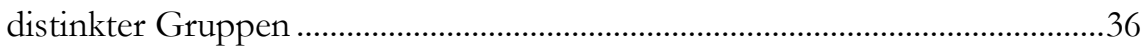

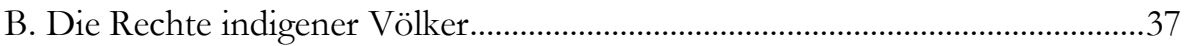

I. Die Geschichte indigener Völker im Völkerrecht ............................................37

II. Rechtsquellen und Institutionen .................................................................41

1. Convention No. 107 der Internationalen Arbeitsorganisation ...............42

2. Convention No. 169 der Internationalen Arbeitsorganisation ...............43

3. UN Declaration on the Rights of Indigenous Peoples .............................46

4. Institutionen ..............................................................................................

5. Zwischenergebnis: eine Kehrtwende der Indigenitätspolitik ..................50 
III. Das Selbstbestimmungsrecht indigener Völker

1. Politische Rechte .52

2. Landrechte. .53

3. Kulturelle und soziale Rechte .54

4. Recht auf Entwicklung 55

5. Zusammenfassung .56

IV. Implementierung und Verfahren ..........................................................56

1. Berichts- und Überwachungsverfahren ..................................................5

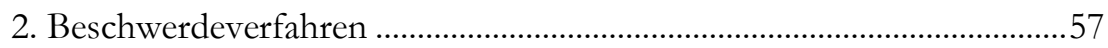

V. Zusammenfassung: globale politische Bewegung

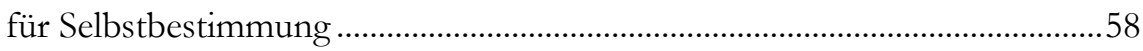

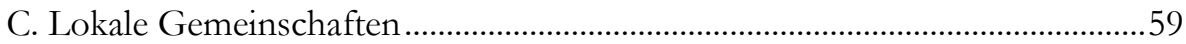

I. Geschichte der indigenen und lokalen Gemeinschaften im Umweltvölkerrecht

1. Umweltrechtliche Regelungen zu substaatlichen Gruppen bis 1992 59

2. Die Rolle traditionellen Wissens .................................................................61

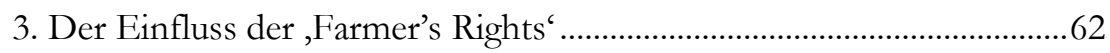

4. Die aktuelle Bedeutung traditionellen Wissens indigener und lokaler Gemeinschaften

II. Rechtsquellen des Konzepts lokaler Gemeinschaften ..............................64

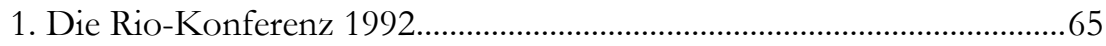

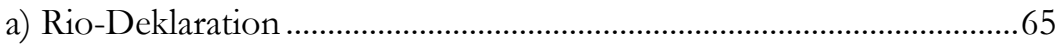

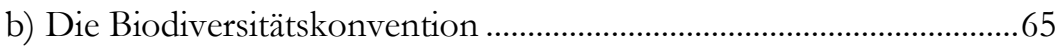

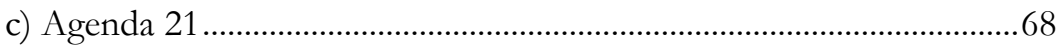

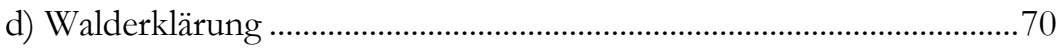

2. Übereinkommen zur Bekämpfung der Wüstenbildung ..........................70

3. World Summit on Sustainable Development .......................................... 71

4. UN Conference on Sustainable Development .........................................72 
5. Verhandlungen zu Rechten geistigen Eigentums und genetischen

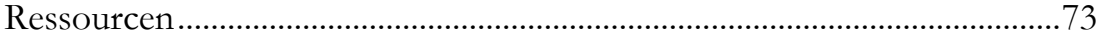

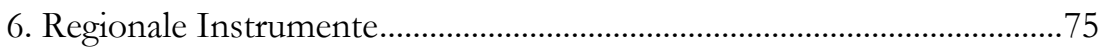

III. Regelungen zum Schutz traditionellen Wissens.........................................76

1. Traditionelles Wissen und access and benefit sharing............................76

2. Staatliche Souveränität über genetische Ressourcen...............................77

IV. Nationalgesetzlicher Rahmen und Rechte geistigen Eigentums ...............78

1. Rechtsdurchsetzungsregelungen im Nagoya Protokoll ............................78

2. Schutz durch Rechte geistigen Eigentums .............................................78

V. Zusammenfassung: neue Akteure beim Schutz von Biodiversität ............79

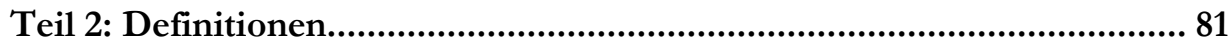

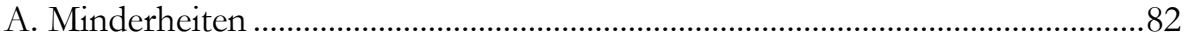

I. Definitionsansatz des Ständigen Internationalen Gerichtshofs .................83

II. Arbeitsdefinitionen der Sonderberichterstatter und weitere

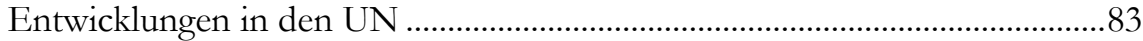

III. Konkretisierung durch den Menschenrechtsausschuss .............................86

IV. Regionale Konkretisierung der Minderheitendefinition ...........................87

V. Verschiedene Elemente einer Minderheitendefinition ..............................87

1. Kulturelle Distinktion und ein, Ausdruck des Gefühls der

Verbundenheit'.

2. Quantitative oder politische Inferiorität ...................................................89

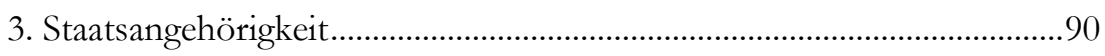

4. Historische und geographische Verwurzelung ........................................ 92

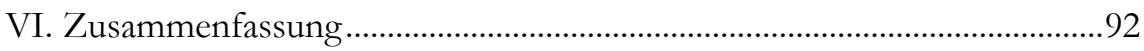

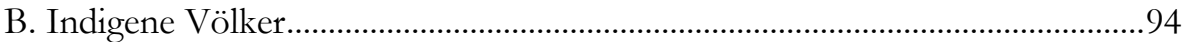

I. Implikationen des Konzepts ,people '...........................................................94

II. Die Definition von José Martínez Cobo .....................................................95

III. Definitionen der ILO Konventionen ......................................................97

IV. Regionale Interpretationen in Asien und Afrika......................................98 
V. Die Deklaration der Vereinten Nationen über die Rechte

indigener Völker 100

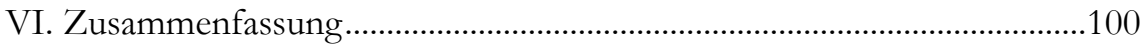

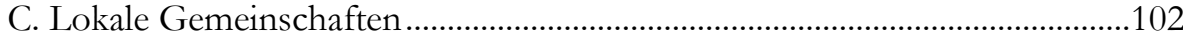

I. Indigene und lokale Gemeinschaften in der CBD ...................................102

II. Unterschiede zwischen indigenous peoples, indigenous communities und local communities ...............................................................103

1. Implizierte Rechte und Ansprüche......................................................104

2. Vergleichbare Praxen und Lebensweisen ..............................................105

3. Kompromiss geographischer Anwendbarkeit ......................................105

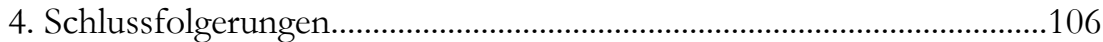

III. Das ad hoc expert meeting of local-community representatives ............106

IV. Geographische Verbreitung lokaler Gemeinschaften.............................109

V. Lokale Gemeinschaften und Farmer's Rights .........................................110

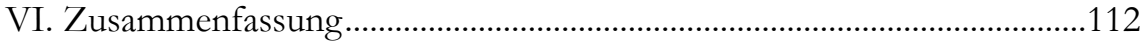

D. Selbstidentifikation kultureller Distinktion als Kern

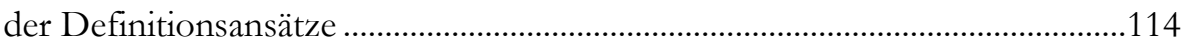

E. Das rechtliche Verhältnis der Konzepte zueinander ....................................116

I. Minderheitenschutz für indigene Völker und lokale Gemeinschaften.....117

II. Die Indigenität von Minderheiten und lokalen Gemeinschaften.............119

III. Lokale Gemeinschaften bestehend aus indigenen Völkern und

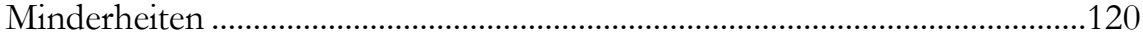

IV. Überlagernde Anwendbarkeit der drei Konzepte...................................121

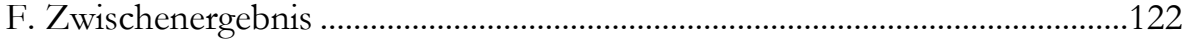

Teil 3: Anerkennung .................................................................125

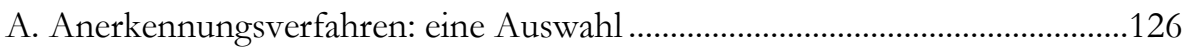

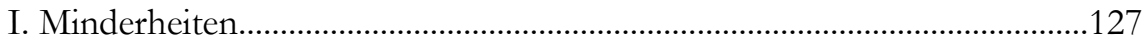

1. Regelungen zu Minderheiten in bilateralen Verträgen ..........................127 
2. Der Einfluss multilateraler Verträge auf die Anerkennung von Minderheiten ................................................................................ 128

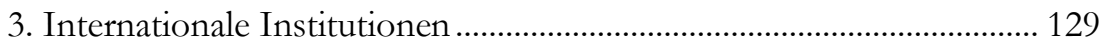

4. Zusammenfassung: die Politik der Anerkennung von Minderheiten

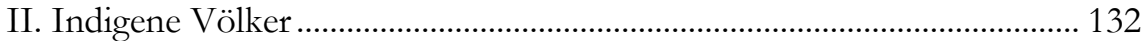

1. Staatliche Anerkennung indigener Völker.............................................. 132

2. Internationale Organisationen und indigene Völker ............................. 134

3. Anerkennung durch NGOs.................................................................... 137

4. Zusammenfassung: international gefestigte Positionen

und geregelte Verfahren für neue indigene Gruppen ................................ 138

III. Lokale Gemeinschaften ............................................................................. 138

1. Anerkennung durch Staaten ................................................................... 139

2. Anerkennung durch internationale Organisationen .............................. 140

3. Zusammenfassung: Anerkennung im Rahmen

umweltrechtlicher Politiken ...................................................................... 142

IV. Akteurskonstellationen der Anerkennung ............................................... 143

1. Staatliche Anerkennung............................................................................. 143

2. Anerkennung durch Drittstaaten ............................................................ 145

3. Internationalisierte Anerkennung …………………………………...... 145

4. Nichtstaatliche Anerkennung ................................................................. 148

V. Zusammenfassung: eine Perspektive der Anerkennung ........................... 148

B. Rechtliche Bewertung der Anerkennung substaatlicher Gruppen .............. 150

I. Anerkennung substaatlicher Gruppen im

Über-/Unterordnungsverhältnis .................................................................. 152

II. Selbstidentifikation und Anerkennung ..................................................... 154

III. Existenz substaatlicher Gruppen und Anerkennung............................... 155

IV. Die Relativität der Anerkennung............................................................ 157

V. Funktioneller Inhalt der Anerkennung durch internationale Akteure... 158 
VI. Politik der Anerkennung .........................................................................158

VII. Problematik verweigerter Anerkennung....................................................159

VIII. Zwischenergebnis ....................................................................................160

C. Interdisziplinäre Perspektiven: Konstruktivismus und Anerkennung .........163

I. Konstruierte Identitäten .............................................................................163

II. Die Aushandlung kollektiver Identitäten und das Anerkennen ...............165

III. Die Wirkmächtigkeit der Zuschreibungen des Völkerrechts ..................167

1. Stereotype Bilder ,indigener Völker ...........................................................169

2. Romantisierte Ideen ,lokaler Gemeinschaften' …………..........................170

3. Die Idealvorstellung von ,Minderheiten' ..................................................170

4. Ergebnis: Holistische Konzepte in den völkerrechtlichen

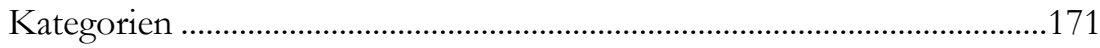

IV. Völkerrechtliche Strukturen und Definitionshoheit .................................173

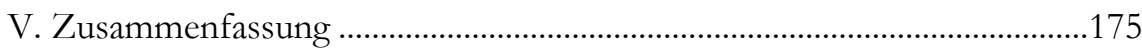

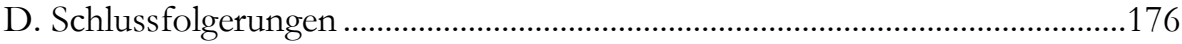

I. Die Anerkennung sollte die Definitionen ersetzen ......................................176

II. Es bedarf Regelungen zu Institutionen und Prozessen

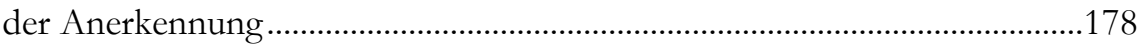

Teil 4: Verhandlungen im WIPO Intergovernmental Committee ..............181

A. Geschichte und Hintergrund.........................................................................182

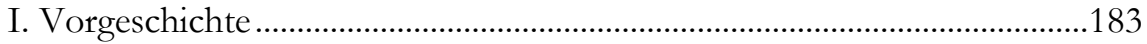

1. Nationale und internationale Entwicklungen im Kontext des

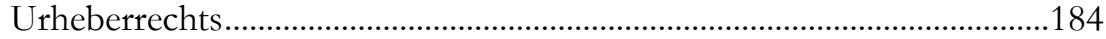

2. Model Laws als sui generis Instrumente auf internationale Ebene.....185

3. Entwürfe multilateraler Instrumente...........................................................186

II. Mandat des Intergovernmental Committee ……………………………......188

III. Verlauf der Verhandlungen des Intergovernmental Committee ...........189

1. Fragebögen zu nationalen Erfahrungen ................................................189

2. Die Draft Provisions (Entwicklungen bis zur 10. Sitzung) ...................189 
3. Die 10 Schlüsselfragen und die Gap Analysis......................................... 190

4. Die Draft Privisions (Weiterentwicklung ab der 15. Sitzung) ............. 191

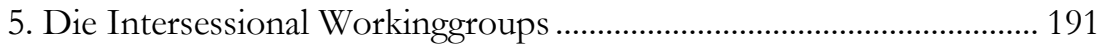

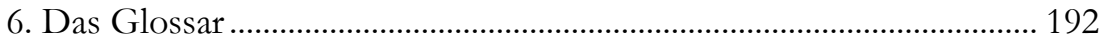

7. Die Draft Provisions (Überarbeitung durch die Intersessional

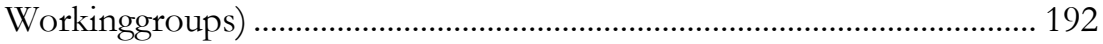

IV. Tabellarische Darstellung der Verhandlungen des WIPO IGC............ 193

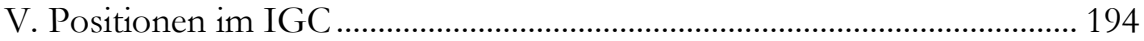

1. Die Gruppe lateinamerikanischer und karibischer Staaten................... 195

2. Die afrikanische Gruppe ………………………………........................ 196

3. Die Gruppe B ................................................................................... 198

4. Die Asien und Pazifik Gruppe und China ............................................. 199

5. Balkan und osteuropäische Staaten sowie zentraleuropäische und baltische Staaten........................................................................................ 199

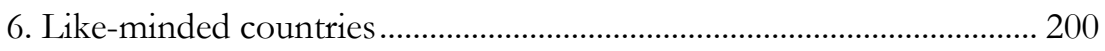

7. Positionen der Vertreter von Nichtregierungsorganisationen und indigener Völker...................................................................................... 201

8. Zusammenfassung des Verlaufs der Verhandlungen

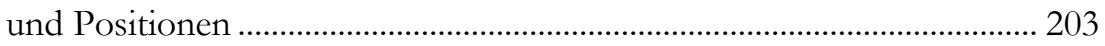

VI. Subject Matter of Protection....................................................................... 203

1. Traditional Cultural Expressions ............................................................ 204

2. Traditional Knowledge ........................................................................... 206

3. Zusammenfassung................................................................................... 207

VII. Durchsetzungsmechanismen.................................................................... 207

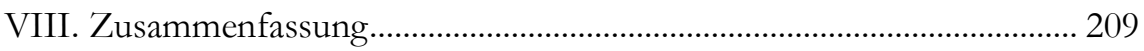

B. Die beneficiaries neuer Instrumente zum Schutz von TK/TCEs................ 211

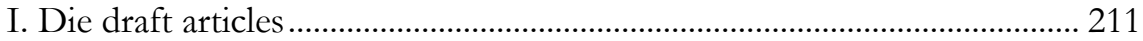

II. Verwendung etablierter Konzepte substaatlicher Gruppen..................... 213

1. ,Indigenous Peoples/Communities '......................................................... 214 
2. ,Local Communities' 216

III. Diskussionen um die Berechtigung der ,Nation“ und die Rolle des Staates

IV. Neue Konzepte: ,Traditional Communities` und ,Cultural

Communities

V. Zusammenfassung 225

C. Die Anerkennung von Berechtigten: die NCA und neue

Akteursverhältnisse 227

D. Zusammenfassung 230

Ergebnis 231

Zusammenfassung in Thesen 235

Bibliographie 241

A. Primärquellen: Verträge, Gesetzgebung, Sitzungsunterlagen, Dokumente, Berichte, Entscheidungen, Nachrichten. 241

B. Sekundärquellen: Monographien, Artikel, Beiträge in Sammelwerken 256 



\section{Abkürzungsverzeichnis}

ABS

CBD

EGMR

EU

FCNM/

Framework Convention

GR

GR/TK/TCEs

GRULAC

IGC

ILO

IPbpR

IWG

Model Provisions

NCA

NGO

OSZE

Rahmenübereinkommen

StIGH

TCE

TK

TK/TCEs

TRIPs

UN

UN CSD

UNDRIPs access and benefit sharing

Convention on Biological Diversity, Biodiversitätskonvention

Europäischer Gerichtshof für Menschenrechte

Europäische Union

Framework Convention for the Protection of

National Minorities, Rahmenübereinkommen zum Schutz nationaler Minderheiten

Genetische Ressourcen

Genetic Resources / Traditional Knowledge /

Traditional Cultural Expressions

Group of Latin American and Caribbean States

Intergovernmental Committee

International Labor Organization

Internationaler Pakt über bürgerliche und politische Rechte

Intersessional Workinggroups

Model Provisions for National Laws on the Protection of Expressions of Folklore

National Competent Authority

Nichtregierungsorganisation

Organisation für Sicherheit und Zusammenarbeit in Europa

Rahmenübereinkommen zum Schutz nationaler Minderheiten, Framework Convention for the Protection of National Minorities

Ständiger Internationaler Gerichtshof

Traditional Cultural Expressions

Traditional Knowledge

Traditional Knowledge / Traditional Cultural Expressions

Trade-Related Aspects of Intellectual Property Rights

United Nations, Vereinte Nationen

United Nations Conference on Sustainable Development

Erklärung der Vereinten Nationen über die Rechte indigener Völker 
xxiv

UNESCO

UNPFII

WGIP

WIPO

WIPO IGC
United Nations Educational, Scientific and Cultural Organization

United Nations Permanent Forum on Indigenous Issues

Working Group on Indigenous Populations

World Intellectual Property Organization

Intergovernmental Committee of the World Intellectual Property Organization 


\section{Einleitung}

\section{A. Substaatliche Gruppen im Völkerrecht}

Das Völkerrecht strukturiert die gesamte Weltbevölkerung als Staatsvölker. Es strukturiert die Weltbevölkerung somit in feste Gruppen, die in Bezug zu ihren Heimatstaaten nach den Grenzen geographisch definierter Gebiete bestimmt werden, wo sie die Gemeinschaft eines gemeinsamen politischen Diskurses bilden. Es strukturiert die Weltbevölkerung in Gruppen, die im Rahmen dieser geographischen Grenzen ihre Anliegen selbst bestimmen können. Dieses System gibt der Welt eine Ordnung, die Frieden schaffen möchte, indem sie Grenzen zieht.

Jedoch ist diese Weltordnung problematisch für Gruppen, die sich kulturell von der dominierenden Mehrheit eines Staates unterscheiden. Aus den kulturellen Unterschiedlichkeiten zwischen substaatlichen Gruppen und der dominierenden Mehrheitsgesellschaft entwickeln sich nicht selten Spannungen und Konflikte. Lösungen solcher Konflikte können substaatliche Gruppen im Diskurs demokratischer Entscheidungen nur ungleich schwerer gegenüber einer dominierenden Mehrheit in ihren Heimatstaaten beeinflussen. Noch schwieriger ist die Lage kulturell distinkter, substaatlicher Gruppen in Staaten, in denen Einschränkungen der Meinungsfreiheit offene Diskussionen und Debatten zur gegenseitigen Verständigung und friedlichen Kompromissfindung erschweren. Nicht selten führten sol- 
A. Substaatliche Gruppen im Völkerrecht

che interkulturellen und ethnischen Konflikte zu gewaltsamen Auseinandersetzungen.

Schon die Beispiele der jüngeren Geschichte zeigen, dass ethnische Konflikte eine entscheidende Ursache gewaltsamer innerstaatlicher und internationaler Auseinandersetzungen sind. Der Krise in der Ukraine wie auch die gewaltsamen Interventionen Russlands in Südossetien und Abchasien machen deutlich, dass auch heute noch Konflikte entlang ethnischer Zugehörigkeiten zu internationalen Auseinandersetzungen heranwachsen können, in denen schließlich geopolitische Großmächte mit militärischen Mitteln die Hoheit über fremdes Territorium zu erlangen und festigen suchen. ${ }^{1}$ Hat ein Aggressor die Intention seinen Gegner vollständig zu vernichten, können ethnische Konflikte die schlimmsten Folgen haben. Allein in den letzten 20 Jahren kam es im Rahmen der gewaltsamen Konflikte in Bosnien, Ruanda sowie in Darfur zum Völkermord an ethnischen Gruppen.

Auch in der Geschichte der Kolonisierung sowie in der bis heute andauernden Bewältigung ihres globalen historischen Erbes offenbaren sich die verheerenden Folgen von Auseinandersetzungen zwischen kulturell unterschiedlichen Gruppen. Erst 2008 entschuldigten sich die Ministerpräsidenten Australiens und Kanadas für die rassistischen Verbrechen im Zuge der aggressiven Assimilierungspolitiken gegenüber den Aborigines und den Torres-Strait-Insulanern beziehungsweise den First Nations Kanadas. ${ }^{2}$ Die Marginalisierung und Unterdrückung indigener Gemeinschaften ist auch heute noch in vielen Teilen der Welt allgegenwärtig und somit Thema politischer Debatten bis hin zu gewaltsamen Auseinandersetzungen.

Im Zuge der Globalisierung ergeben sich zudem ökonomische Auseinandersetzungen über die Zuordnung an kulturellen Hervorbringungen. Das ökonomische Potential traditionellen Wissens und traditioneller kultureller Ausdrucksformen, der Wert von Wissen und Praxen, das kulturelle Eigentum, die alle aus kultureller Unterschiedlichkeit hervorgehen, werfen neue Fragen auf. Gruppen, deren Kultur durch Dritte angeeignet und kommerzialisiert wird, sprechen hier von Diebstahl und erheben Ansprüche auf eine in Zukunft notwendige Zustimmung zur Nutzung sowie auf Teilhabe an den Gewinnen. Dabei stehen sich meist kulturell distinkte, rechtlich nicht verfasste Gruppen aus dem ,globalen Süden' und globale Unternehmen aus dem, Westen' gegenüber.

\footnotetext{
${ }^{1}$ Peter W. Schulze, „Geopolitics at Work: the Georgian-Russian Conflict“, Goettingen Journal of International Law, Russia and International Law - From the North Pole to the Caucasus, Vol. 1, Nr. 2 (2009), S. 329; Angelika Nußberger, „The War between Russia and Georgia - Consequences and Unresolved Questions", Goettingen Journal of International Law, Russia and International Law From the North Pole to the Caucasus, Vol. 1, Nr. 2 (2009), S. 341.

2 „Ureinwohner in Australien - Historische Entschuldigung“, Süddeutsche Zeitung, 17. Mai 2010; Matthias Rüb, „Harper entschuldigt sich bei Ureinwohnern: Kanada weint nach der historischen Geste“, Frankfurter Allgemeine Zeitung, 6. Dezember 2008.
} 
Das Konfliktpotential zwischen kulturell unterschiedlichen Gruppen ist dem völkerrechtlichen System inhärent: Es sind die Grundprinzipien des Völkerrechts, die Struktur der Aufteilung der Welt in Staaten, die Prinzipien der Souveränität und territorialen Integrität, welche die Rahmenbedingungen schaffen, in der eine dominierende Position der Mehrheitsgesellschaft gegenüber kleineren Gruppen zu Problemen führen kann. ${ }^{3}$ Das Völkerrecht schafft Strukturen für Gruppen von Menschen und kategorisiert damit die Formen ihrer Repräsentation, so dass Vertreter von nicht-staatlichen Akteuren in ihrer Position zurückgesetzt sind. Um der strukturellen Benachteiligung substaatlicher Gruppen auf nationaler und internationaler Ebene zu begegnen, haben sich jedoch mit der Zeit im Völkerrecht verschiedene Konzepte und Instrumente entwickelt, die diesen Gruppen einen gesonderten Status respektive gewisse Sonderrechte zugestehen. Mit Hilfe dieser Instrumente soll für Wahrnehmung, Ausgleich und Schutz substaatlicher Gruppen im internationalen System der Staaten sowie innerstaatlich gegenüber dominierenden Mehrheitsgesellschaften gesorgt werden. Letztlich sollen diese Instrumente für Ausgleich sorgen und friedensstiftend wirken.

\section{Die bestehenden Konzepte substaatlicher Gruppen}

Die völkerrechtlichen Instrumente mit Regelungen zu kulturell distinkten, substaatlichen Gruppen lassen sich in drei Konzepte kategorisieren: Minderheiten, indigene Völker und lokale Gemeinschaften. Die Instrumente des internationalen Minderheitenschutzes garantieren beispielsweise die Freiheit der Ausübung der eigenen Religion und Sprache, um Minderheiten in ihrer kulturellen Integrität zu schützen. Die Vertreter der Rechte indigener Völker proklamieren den umfassenden Anspruch auf Selbstbestimmung, nach welchem indigene Gemeinschaften ihre politische, wirtschaftliche, soziale und kulturelle Entwicklung selbst gestalten können sollen, wobei der Rahmen staatlicher Grenzen nicht berührt werden soll. Im internationalen Umweltrecht sind seit jeher gewisse Ausnahmeregelungen von Artenschutzvorschriften zu Gunsten lokaler Gemeinschaften und ihren verschiedenen traditionellen kulturellen Praxen anerkannt. Im internationalen Umweltrecht wurden jüngst weitere normative Strukturen zum Zugang und zur Vorteilsteilhabe an pflanzengenetischen Ressourcen und assoziiertem traditionellen Wissen lokaler Gemeinschaften entwickelt. Hintergrund all dieser Konzepte ist in unterschiedlichen Formen der Schutz der Kultur substaatlicher Gruppen.

\footnotetext{
${ }^{3}$ So die grundsätzliche Kritik am europäischen Völkerrecht der Third World Approaches to International Law (TW AIL). Einen grundlegenden Überblick dieser kritischen Perspektive findet sich bei: Antony Anghie, Imperialism, sovereignty and the making of international law, 2005.
} 


\section{Das Problem einer rechtlichen Definition von kulturell distinkten Gruppen}

Bisher konnten sich weder die internationale Staatengemeinschaft noch die Wissenschaft auf eine internationale, allgemein anerkannte, positivrechtliche Definition kulturell distinkter, substaatlicher Gruppen einigen. ${ }^{4}$ Trotz der Vielzahl von Versuchen zur Erarbeitung von Definitionen in unterschiedlichen Arbeitsgruppen, trotz verschiedener Ansätze in sogenannten Arbeitsdefinitionen von UNSonderberichterstattern und trotz einer entsprechenden kritischen Begleitung und Reflexion in der Wissenschaft einigte man sich bei internationalen Verhandlungen bisher stets auf die Kompromisslösung einer individuellen Ausgestaltung der Begrifflichkeiten auf nationaler Ebene. Es entwickelten sich somit unterschiedliche Vorgehensweisen bei der Anwendung der Konzepte substaatlicher Gruppen auf nationaler Ebene.

Zudem sind die drei bestehenden völkerrechtlichen Konzepte substaatlicher Gruppen ,Minderheit', ,indigenes Volk ${ }^{6}$ und ,lokale Gemeinschaft' in ihren jeweiligen Regelungsbereichen kontextualisiert, so dass sich die Frage stellt, ob und inwiefern diese Konzepte je unterschiedliche Typen substaatlicher Gruppen definieren. Auf Grund der jeweils eigenen Geschichte, der unterschiedlichen Regelungskontexte und den jeweils verknüpften andersartigen Ansprüchen und Rechten ließe sich annehmen, sie stünden in einem Verhältnis sich ausschließender Exklusivität zueinander.

Unter einer vergleichenden Perspektive erkennt man jedoch, dass alle bisherigen Ansätze abstrakt-genereller Definitionen der drei Konzepte im Kern auf eine ,Selbstidentifikation kultureller Distinktion' der Gruppe rekurrieren. Betrachtet man diesen gemeinsamen Kern der Definitionsansätze getrennt von den jeweiligen Regelungskontexten und historisch gewachsenen Eigenheiten der unterschiedlichen Konzepte, bestätigt sich die These Ian Brownlies:

The heterogeneous terminology which has been used over the years - the references to nationalities, peoples, minorities and indigenous peoples - involves essentially the same idea. ${ }^{5}$

Trotz der verschiedenartigen Terminologie besteht demnach für alle Konzepte dieselbe Kernidee, was eine kulturell distinkte Gruppe ausmacht. Wenn sich nun alle Konzepte substaatlicher Gruppen im Kern auf ,dieselbe Idee“ im Sinne Ian Brownlies beziehen, bedeutet dies in der Konsequenz, dass alle substaatlichen Gruppen diese Voraussetzung einer rechtlichen Definition erfüllen und somit

\footnotetext{
${ }^{4}$ Die einzige Ausnahme einer Definition substaatlicher Gruppen ist für den Begriff 'indigene Völker' die Konvention Nr. 169 der ILO, die mit nur 22 Ratifikationen vornehmlich im südamerikanischen Raum, global allerdings keine allgemeine Verbreitung gefunden hat. Mehr dazu unter: „B. III. Definitionen der ILO Konventionen" auf S. 97.

${ }^{5}$ Ian Brownlie, ,The Rights of Peoples in Modern International Law“, in: James Crawford (Hrsg.), The Rights of Peoples, 1988, S. 5.
} 
gleichzeitig auch der Kernvoraussetzung der anderen Konzepte genügen. Ein und dieselbe Gruppe, die sich durch die Selbstidentifikation ihrer kulturellen Distinktion als Kollektiv konstituiert, erfüllt somit den Kerngehalt aller drei Konzepte substaatlicher Gruppen. Aus einer rechtlichen Perspektive betrachtet können Gruppen demnach auch unter die weiteren Konzepte subsumiert werden, sofern dafür die weiteren historisch gewachsenen notwendigen Voraussetzungen vorliegen.

Zusammenfassend zeigt sich somit folgende Problemstellung: Weder in der Staatengemeinschaft noch in der Wissenschaft konnte man sich bisher auf eine abstrakt-generelle Definitionen einigen, mit Hilfe derer eine differenzierbare Anwendung der Konzepte substaatlicher Gruppen rechtlich geregelt werden kann. 


\section{B. Grundlagen und Ziel der Untersuchung}

\section{Gegenstand und Forschungsfrage}

Gegenstand der vorliegenden Untersuchung sind die drei Konzepte substaatlicher Gruppen im Völkerrecht, wie sie sich in den Instrumenten des internationalen Minderheitenschutzes, den Entwicklungen zu den Rechten indigener Völker und den Normen zu lokalen Gemeinschaften im Umweltvölkerrecht niederschlagen. Für diese Konzepte stellt sich die hier grundlegende Forschungsfrage: Wie lassen sich kulturell distinkte Kollektive rechtlich bestimmen, um die völkerrechtlichen Konzepte substaatlicher Gruppen zur Anwendung zu bringen? Zur Beantwortung dieser Frage werden die dafür bisher vorgeschlagenen unterschiedlichen Definitionsansätze vorgestellt und analysiert. Ausgehend von der Praxis der Anwendung der Konzepte wird als Alternative eine Perspektive der Anerkennung vorgestellt. Mit Hilfe dieser neuen Perspektive der Anerkennung lassen sich die Prozesse und Institutionen rechtlich einordnen, die notwendig sind, um die Rechte von Minderheiten, indigenen Völkern oder lokalen Gemeinschaften umzusetzen. Dabei wird insbesondere auf die völkerrechtliche Debatte zur Staatenanerkennung vergleichend zurückgegriffen. Zudem dient der Vorschlag einer national competent authority (NCA) zur Wahrnehmung von Rechten substaatlicher Gruppen, wie sie gegenwärtig in den Verhandlungen zu neuen Gruppenrechten an kulturellen Ausdrucksformen diskutiert wird, als Ansatzpunkt neuer völkerrechtlicher Institutionen zur Verrechtlichung der Anerkennung, sprich der Anwendung der Konzepte substaatlicher Gruppen.

\section{Gang der Untersuchung}

Die Untersuchung gliedert sich in vier Teile: Zuerst soll eine Einführung die drei bestehenden Gruppenrechtskonzepte darstellen. Im zweiten Teil folgt eine eingehende Untersuchung der unterschiedlichen Definitionsansätze der Konzepte. Der dritte Teil befasst sich mit den Prozessen der Anwendung der Konzepte und stellt auf dieser Grundlage eine neue Perspektive der Anerkennung vor, die einer rechtlichen Bewertung und kritischen Analyse der aktuellen Rechtslage dient. Teil 4 beleuchtet die gegenwärtigen Verhandlungen zu neuen Gruppenrechten geistigen Eigentums im Intergovernmental Committee der Weltorganisation für geistiges Eigentum (WIPO IGC) und zeigt, dass hier die als notwendig erachtete internationale Institutionalisierung der Anerkennung substaatlicher Gruppen in Form der NCA geschaffen werden könnte. 


\section{Konzepte substaatlicher Gruppen}

Die Wurzeln des internationalen Minderheitenschutzes finden sich in Form unilateraler Erklärungen bereits im 13. Jahrhundert bei Auseinandersetzungen zwischen Christen und Muslimen. Mit den politischen Ereignissen im Zuge der protestantischen Reformation des 16. Jahrhunderts wurde der Schutz der ,Andersgläubigen' erstmals auch bi- und multilateral auf internationaler Ebene rechtlich verankert. Die Entwicklung des internationalen Minderheitenschutzes erreichte ihren Höhepunkt nach Ende des Ersten Weltkriegs im Zuge der Neuordnung der Staaten Europas. Es bildete sich eine Vielzahl von Exklaven nationaler Minderheiten, die zur Zeit des Völkerbundes Schutzrechte bezüglich ihrer Sprache, Religion und Kultur erhielten. Folge der politischen Debatten nach den verheerenden Ereignissen des Zweiten Weltkriegs war die schwindende Bedeutung des kollektiven Minderheitenschutzes gegenüber dem internationalen individualrechtlichen Menschenrechtssystem. Heute bildet neben einer Vielzahl von bilateralen und einigen multilateralen Verträgen in Europa insbesondere Artikel 27 des Internationalen Pakts über bürgerliche und politische Rechte (IPbpR) ${ }^{6}$ die tragende normative Grundlage eines Gruppenkonzepts für Minderheiten im globalen System der internationalen Menschenrechte.

Die rechtliche Konzeptualisierung indigener Völker begann mit der Besiedelung der ,Neuen Welt' vor über 500 Jahren. Anfangs dienten die Theorien zum Verlust des dominium der Indigenen über ihr Land sowie die Argumente eines terra nullius als Rechtfertigung der Bekämpfung und Unterdrückung der Ureinwohner. Sie garantierten dabei gleichzeitig eine effektive Aufteilung des neuen Landes unter den Europäern. Seit der Ära der Vereinten Nationen und insbesondere seit den 1970er Jahren sprechen indigene Völker auf internationaler Ebene zunehmend mit eigener Stimme. Ihr Anspruch auf Selbstbestimmung wurde nach langen Verhandlungen 2007 in der UN Declaration on the Rights of Indigenous Peoples (UNDRIPs) durch die UN Generalversammlung festgeschrieben.

Zusätzlich zum Konzept der indigenen Völker mit seinem völkerrechtlich speziell geprägten Begriffsteil people findet seit Ende des 20. Jahrhunderts im internationalen Umweltvölkerrecht der Begriff ,lokale Gemeinschaft ${ }^{\star}$ Verwendung. Er verknüpft die Entwicklungen der Rechte indigener Völker mit internationalen Regelungen von Bauernrechten. Insbesondere Artikel 8(j) der Convention on Biological Diversity $(C B D)^{7}$ normiert unter dem Begriffspaar der ,indigenen und lokalen Gemeinschaft' die Bedeutung dieses Konzepts im modernen Umweltvölkerrecht und konkretisiert die Rolle lokaler Gemeinschaften in Bezug auf pflanzengenetische Ressourcen und assoziiertes traditionelles Wissen.

\footnotetext{
${ }^{6}$ International Covenant on Civil and Political Rights (Internationaler Pakt über die bürgerlichen und politischen Rechte, IPbpR), 999 UNTS 171, 16. Dezember 1966.

${ }^{7}$ Convention on Biological Diversity (Übereinkommen über die biologische Vielfalt, CBD), 1760 UNTS 79, 5. Juni 1992.
} 
B. Grundlagen und Ziel der Untersuchung

\section{Definitionsansätze substaatlicher Gruppen}

Bei keinem der Konzepte substaatlicher Gruppen konnte man sich bisher auf eine allgemein anerkannte, abstrakt-generelle, juristische Definition der geschützten bzw. mit Rechten ausgestatteten Gruppe einigen. Vor dem Hintergrund der unterschiedlichen Regelungskontexte und der historischen Entwicklungen der verschiedenen Konzepte wurden jeweils Ansätze von Definitionen entwickelt.

Teil 2 der Untersuchung stellt dar, dass allen Konzepten ein grundlegend vergleichbarer Ansatz eines Ausdrucks des Zusammengehörigkeitsgefühls und der Selbstidentifikation bezüglich der kulturellen Distinktion aufgrund von Sprache, Religion oder sonstigen kulturellen Praxen der Gruppen zu eigen sind. Bei den Minderheitenschutzinstrumenten in Europa werden zusätzlich die Staatsbürgerschaft des Staates, in dem die Minderheit lebt, sowie eine historische und geographische Verwurzelung vorausgesetzt. Indigene Völker sind solche Gruppen, die eine Verbindung zu präkolonialen Gesellschaften aufweisen. Zusätzlich erfuhr das Konzept für seine Anwendbarkeit in Afrika und Asien eine Uminterpretation und Fokussierung auf eine politische, wirtschaftliche und soziale Marginalisierung und Unterdrückung der Gruppe. So artikulieren indigene Völker auch in diesen Regionen nun Ansprüche auf Selbstbestimmung. Und schließlich genießen Gruppen als lokale Gemeinschaften die umweltvölkerrechtlichen Zustimmungs- und Teilhaberechte, sofern sie nachhaltigen, traditionellen und subsistenzwirtschaftlichen Praxen folgen.

Der gemeinsame Kern der kollektiven Selbstidentifikation bezüglich einer kulturellen Distinktion aller drei Konzepte bildet die Grundlage für Ausführungen zum rechtlichen Verhältnis der Konzepte zueinander und ihrer überlagernden Anwendbarkeit. Aus rechtlichen Gründen spricht nichts dagegen, dass substaatliche Gruppen gleichzeitig unter einem oder mehreren der drei Konzepte agieren.

Trotz der Vielzahl der diskutierten Ansätze konnte sich bisher keine abstraktgenerelle Definition durchsetzen. Grund dafür sind nicht nur die aus politischen Gründen unterschiedlich eng oder weit gefassten verschiedenen Elemente der Definitionsansätze. Problematisch ist, dass die Selbstidentifikation kultureller Distinktion, wie sie allen Konzepten als gemeinsame Idee zu Grunde liegt, als einseitige Erklärung seitens der substaatlichen Gruppe als unpraktikabel und dem Missbrauch offen abgelehnt wird. Die Frage, ob und für wen die internationalen Konzepte substaatlicher Gruppen für kulturell distinkte Kollektive zur Anwendung kommen, ist rechtlich nach wie vor ungeklärt.

\section{Anerkennung}

Betrachtet man die Umsetzung internationaler Instrumente zum Schutz substaatlicher Gruppen auf nationaler und internationaler Ebene, so wird deutlich, dass sie erst zur Anwendung kommen, wenn Gruppen als ,Minderheit', indigenes Volk ${ }^{\varsigma}$ oder ,indigene und lokale Gemeinschaft ${ }^{\star}$ anerkannt werden. Ange- 
sichts der bisher nicht konsentierten juristischen Definitionsansätze soll diese Anerkennung hier als neue Perspektive eines rechtlichen Verständnisses vorgestellt werden. Die Anerkennung bildet das Pendant zur Selbstidentifikation einer Gruppe. Im Gegensatz zur deklaratorischen völkerrechtlichen Staatenanerkennung kommt der Anerkennung substaatlicher Gruppen für die rechtliche Anwendbarkeit der unterschiedlichen Konzepte eine konstitutive Bedeutung zu. Zwar entwickelt sich bei den Fragen der Anerkennung immer ein komplexes Zusammenspiel einer Vielzahl beteiligter Akteure, die letztliche Entscheidungshoheit obliegt jedoch hauptsächlich den Staaten, in denen die jeweiligen Gruppen leben. Es zeigt sich, dass die gegenwärtige ungeklärte Rechtslage Machtungleichgewichte zwischen substaatlichen Gruppen und ihren Heimatstaaten reproduziert, die eigentlich durch die völkerrechtlichen Instrumente ausgeglichen werden sollen.

Schließlich wird gezeigt, dass die internationalrechtlichen Instrumente eine entscheidende Rolle bei der Genese kollektiver Identitäten spielen. Kollektive positionieren sich unter Rückgriff auf die völkerrechtlichen Konzepte substaatlicher Gruppen gegenüber Dritten, insbesondere auch gegenüber den Regierungen ihrer Heimatstaaten, im Lichte entsprechender Narrative. Die interdisziplinären Einsichten unter Rückgriff auf die Kulturwissenschaften offenbaren, dass die bestehenden internationalen Instrumente mit ihren jeweils holistischen Konzepten eine nur unzureichende Gestaltungsmöglichkeit der Konstruktionen kollektiver Identität zur Verfügung stellen, um der Vielfalt sowie dem ständigen Wandel und den Veränderungen von Kulturen gerecht zu werden. Im Lichte der politischen Theorie wird zudem deutlich, dass die Verfahren, welche die Anerkennung substaatlicher Gruppen normieren möchten, einer Beteiligung substaatlicher Gruppen und der Mehrheitsgesellschaft im öffentlichen politischen Dialog der Aushandlung kollektiver Identität gerecht werden müssen. Nur so kann der ,verkennenden Anerkennung ${ }^{6}$ begegnet werden, die sich durch die Projektionen des Bildes und der Zuschreibungen der Mehrheit gegenüber ,den Anderen' der substaatlichen Gruppe vollzieht. Daraus ergeben sich Vorschläge für Regelungen de lege ferenda.

\section{Die Verhandlungen im IGC der WIPO}

In Bezugnahme auf die bereits geprägten Konzepte und unter Verwendung neuer Begrifflichkeiten werden gegenwärtig in den Verhandlungen des WIPO IGC kollektivrechtliche Instrumente geistigen Eigentums zum Schutz von genetischen Ressourcen, traditionellem Wissen und traditionellen kulturellen Ausdrucksformen (GR/TK/TCEs) entwickelt. Durch solche neuen privatrechtlichen Instrumente, so die Überlegungen, sollen kulturelle Vielfalt geschützt und gefördert sowie die Trägergruppen von GR/TK/TCEs als ,Berechtigte، unmittel-

\footnotetext{
8 Thomas Bedorf, Verkennende Anerkennung: Über Identität und Politik, 2010.
} 
bar oder mittelbar mit eigenen Rechten ausgestattet werden. Neben einer Darstellung des Verhandlungsverlaufs und der unterschiedlichen Positionen der beteiligten Staatengruppen werden die gegenwärtigen Entwürfe der draft articles bezüglich ihrer Regelungen zu Gruppen der Berechtigten untersucht. In Hinblick auf die Schaffung einer national competent authority, die als zentrale Regierungsbehörde oder als internationale Institution die Rechtsausübung der Berechtigten unterstützen soll, werden neue Akteurs- und Interessenkonstellationen offenbar: Einerseits kann dadurch die Anerkennung substaatlicher Gruppen gefördert werden, andererseits wird der Einfluss und die Kontrolle von staatlicher Seite verstärkt.

\section{Ziel der Untersuchung}

Der rechtliche Status substaatlicher Gruppen spielt eine entscheidende Rolle bei einer juristischen Bewältigung interkultureller Konflikte. Vor diesem Hintergrund soll die vorliegende Arbeit einen Beitrag zu der Frage leisten, wie kulturell distinkte Gruppen rechtlich greifbar gemacht werden können. Nur durch solch eine Bestimmung der Gruppe - und diese Problematik stellt sich gleichermaßen für alle völkerrechtlichen Konzepte substaatlicher Gruppen - kann ihr ein rechtlicher Status zuteil werden. Der bisher dominierende Fokus der Debatte zur Bestimmung von ,Minderheiten', indigenen Völkern` und ,lokalen Gemeinschaften' soll dazu von den Ansätzen abstrakt-genereller Definitionen auf den Prozess der Anerkennung verschoben werden. Es soll gezeigt werden, dass unter der gegenwärtigen Rechtslage die Anerkennung substaatlicher Gruppen den entscheidenden - aber bisher völkerrechtlich nicht geregelten - Schritt zur Anwendung von Minderheitenrechten, dem Selbstbestimmungsrecht und den Regelungen zum access and benefit sharing bildet.

Der umfassende Überblick über die völkerrechtlichen Konzepte substaatlicher Gruppen, ihrer Rechtsquellen, rechtlichen Gehalte und ihrer Durchsetzungs- beziehungsweise Überwachungs- und Berichtsmechanismen bildet die Grundlage für die Analyse der gegenwärtig diskutierten Definitionen. Er soll nur skizzenhaft die groben Strukturen der Konzepte darstellen, da sich detaillierte Ausführen bereits in zahlreichen Einzeldarstellungen finden, auf die entsprechend beleghaft verwiesen wird. Aus der eingehenden Analyse der unterschiedlichen Ansätze der Definitionen wird dann der gemeinsame Kern der ,Selbstidentifikation kultureller Distinktion' herausgearbeitet, welcher den Ausgangspunkt der rechtlichen Argumentation zur überlagernden Anwendbarkeit der Konzepte bildet, die als solche auch durch die völkerrechtliche Praxis bestätigt wird.

Schließlich werden beispielhaft einige Anerkennungsverfahren dargestellt, um dadurch die Perspektive der Anerkennung zu erarbeiten. Unter Rückgriff auf die Debatte der Staatenanerkennung wird sie juristisch eingeordnet und ihr konstitutiver Charakter belegt. Gerade in Anbetracht der Machtungleichgewichte zu Lasten substaatlicher Gruppen sei darauf hingewiesen, dass diese Darstellung kein Plädo- 
yer für die konstitutive Wirkung darstellt. Es ist vielmehr „nur“ das Ergebnis der wissenschaftlichen Analyse der bestehenden Rechtslage.

Als Lösungsansatz wird auf den Vorschlag einer, internationalen Institution der Anerkennung 'verwiesen, wie ihn bereits die Sonderberichterstatterin der UN Working Group on Indigenous People Erica-Irene Daes in die Debatte einbrachte. Weiterführende Vorschläge de lege ferenda werden auf Grundlage der interdisziplinären Perspektiven erarbeitet: Zum einen sollten die holistischen Konzepte differenzierbar ausgestaltet werden können, um einzelne Praxen unter rechtlichen Schutz stellen zu können. Zum anderen bedarf es der Beteiligung der substaatlichen Gruppen und der Mehrheitsbevölkerung im Prozess der Aushandlung kollektiver Identität, um der ,verkennenden Anerkennung' begegnen zu können.

Die Analyse der gegenwärtigen Verhandlungen des WIPO IGC folgt dem Muster der Darstellung der Konzepte aus Teil 1 mit einem besonderen Fokus auf die Verhandlungspositionen der einzelnen Staatengruppen. Diese Darstellung bildet die Grundlage für eine eingehende Untersuchung der draft articles in Hinblick auf die angedachte Normierung der beneficiaries neuer Rechte an TK/TCEs. Auch wenn hier im Ergebnis dieselben Konzepte substaatlicher Gruppen mit den damit einhergehenden Problemen der Anerkennung wiederkehren, könnte sich durch Schaffung der national competent authority eine grundlegende Veränderung der Akteurs- und Interessenkonstellation vollziehen. Im Gegensatz zu den in Teil 1 für die bestehenden Konzepte jeweils dargestellten bipolaren Konstellationen ,substaatlicher Gruppen vs. ihrer Heimatstaaten' entstünde ein neues Drei-AkteursVerhältnis: Substaatliche Gruppen könnten zusammen mit ihren Heimatstaaten ihre Rechte an TK/TCEs gegenüber potentiellen Nutzern im - zumeist ,westlichen' - Ausland geltend machen.

Zusammenfassend lässt sich das Ziel der vorliegenden Arbeit folgendermaßen beschreiben: Es gilt die gegenwärtige Rechtslage der Definitionsansätze sowie die Praxis der Anwendung der Konzepte substaatlicher Gruppen zu erfassen, rechtlich zu analysieren und mit Hilfe interdisziplinärer Perspektiven kritisch zu reflektieren. Der Fokus der Debatte über die Bestimmung substaatlicher Gruppen soll dabei von den statischen Definitionsansätzen auf den Prozess der Anerkennung dieser Gruppen gelenkt werden. Schließlich sollen die gegenwärtigen Verhandlungen des WIPO IGC kritisch kommentiert, die neuen Entwicklungen zu internationalen Gruppenrechten im Kontext der bestehenden Konzepte substaatlicher Gruppen verortet sowie die Chancen neuer internationaler Institutionen der Anerkennung ausgeleuchtet werden. 



\section{Teil 1: Substaatliche Gruppen im Völkerrecht}

Im Völkerrecht finden sich verschiedene Entwicklungen, die mit unterschiedlichen Begriffen und Konzepten Regelungen für substaatliche Gruppen normieren. Diese Konzepte lassen sich vereinfacht in drei Entwicklungslinien strukturieren: den internationalen Minderheitenschutz, die Entwicklungen zu Rechten indigener Völker und die zunehmende Bedeutung lokaler Gemeinschaften im Umweltvölkerrecht. Jeder dieser drei Bereiche hat seine eigene Geschichte und lässt sich trotz der globalen Anwendbarkeit der jeweils zu Grunde liegenden normativen Quellen in unterschiedlichen Regionen der Welt verorten.

\section{A. Der internationale Minderheitenschutz}

Der internationale Minderheitenschutz fußt auf einer jahrhundertealten Geschichte und umfasst eine Vielzahl unterschiedlicher Rechtsquellen. Er steht im Kontext des Systems der internationalen Menschenrechte und hat somit Anteil an dessen Berichts- und Beschwerdemechanismen. Dadurch erfährt er im modernen Völkerrecht eine zunehmende Verrechtlichung sowie eine fortwährende Konkretisierung der Ausgestaltung seiner Schutzgehalte. 
I. Geschichte des Minderheitenschutzes im Völkerrecht

Die ersten Vorläufer des internationalen Minderheitenschutzes entwickelten sich bereits im 13. Jahrhundert im Kontext unilateraler Erklärungen zum Schutz religiöser Minderheiten zwischen Christen und Muslimen im vorderen Orient. ${ }^{9}$ Multilaterale Minderheitenschutzpflichten zwischen Staaten wurden erstmals mit der Verabschiedung des Westfälischen Friedens 1648 garantiert. ${ }^{10}$ Hintergrund dieser Garantien ist die protestantische Reformation mit den darauf folgenden Glaubenskriegen des 16. und 17. Jahrhunderts, die mit dem Augsburger Religionsfrieden von 1555 beendet wurden. ${ }^{11}$ Der Augsburger Religionsfrieden normierte damals den Grundsatz cuius regio, eius religio, nach dem die Reichsstände die Religionszugehörigkeit ihrer Untertanen bestimmen konnten, was zu entsprechenden Veränderungen der konfessionellen Zugehörigkeit verschiedener Landesteile führte. ${ }^{12}$ Mit dem Westfälischen Frieden 1648 und weiteren darauf folgenden Friedensverträgen bis hinein ins 19. Jahrhundert wurden bei Gebietsveränderungen und daraus resultierenden Umverteilungen bezüglich der konfessionellen Zusammensetzung der Bevölkerung eines Reiches Regelungen getroffen, die den Untertanen anderer Konfessionen die Ausübung ihrer Religion gestatten sollten. ${ }^{13}$ In der Schlussakte des Wiener Kongresses 1815 finden sich die Auswirkungen des aufkommenden Nationalstaatsdenkens in Form von Regelungen zum Schutz von nationalen Minderheiten. ${ }^{14}$ Neben Rechten zur Abwanderung wurden darin auch eine nationale Repräsentation und Institutionen für nationale Minderheiten garantiert. ${ }^{15}$

Den Höhepunkt seiner Anerkennung und Verbreitung erlebte der internationale Minderheitenschutz in der Zeit nach dem Ersten Weltkrieg. Damals bestimmte der von Woodrow Wilson proklamierte Grundsatz des ,Selbstbestimmungsrechts der Völker` die Neuordnung und Grenzziehung Europas, die nicht nur neue staatliche Gemeinwesen, sondern auch viele substaatliche Gruppen nationaler Minderheiten schuf. Für diese Gruppen wurde ein allgemeiner Minderhei-

\footnotetext{
${ }^{9}$ Patrick Thornberry, International law and the rights of minorities, 1991, S. 25-27; Hugo Wintgens, Der völkerrecbtliche Schutz der nationalen, spracblichen und religiösen Minderbeiten, 1930, S. 57-61.

${ }^{10}$ Ludwig XIV und Ferdinand III, Westfälischer Friede - Vertrag von Münster (Instrumentum Pacis Monasteriensis), 1649.

11 „Augsburger Religionsfrieden“, in: Rosemarie Aulinger u. a. (Hrsg.), Deutsche Reichstagsakten: Deutsche Reichstagsakten unter Kaiser Karl V. Der Reichstag zu Augsburg 1555, Jüngere Reihe Bd. 20, Teilbd. 4, 2009.

12 Jay A. Sigler, Minority rights, 1983, S. 55.

${ }_{13}$ Christian Scherer-Leydecker, Minderbeiten und sonstige etbnische Gruppen: eine Studie zur kulturellen Identität im Völkerrecht, 1997, S. 30.

${ }^{14}$ Schlussakte der Wiener Ministerkonferenzen oder Bundes-Supplementar-Akte (,Wiener Schlussakte“), 25. November 1918, in: Karl Binding (Hrsg.), Deutsche Staatsgrundgesetze, Teil 3: Konföd.-, Deutsche Bundes-, Wiener Schlußakte, 1909.

${ }^{15}$ Scherer-Leydecker, Minderheiten und sonstige ethnische Gruppen, S. 32.
} 
tenschutz bereits in den Verhandlungen zur Gründung des Völkerbunds diskutiert. ${ }^{16}$ Eine rechtliche Regelung war insbesondere deswegen notwendig geworden, weil die Proklamation der ,Selbstbestimmung der Völker ${ }^{6}$ auch Wünsche und Erwartungen bei den Minderheitengruppen weckte, sich in politischer Hinsicht nicht den Mehrheiten unterwerfen zu müssen. Die Folge waren Spannungen durch Unabhängigkeitsbewegungen auf Seiten der Minderheiten und Tendenzen einer kulturellen Assimilierung auf Seiten der Mehrheiten. ${ }^{17}$ Eine Einigung auf einen allgemeinen Minderheitenschutz im Rahmen des Völkerbundes konnte dennoch nicht erzielt werden. Dem credo der britischen Delegation folgend etablierte sich der Schutz ethnischer Gruppen jeweils in den betroffenen Staaten: teils als eigenständige unilaterale Erklärung, teils im Rahmen der in den Friedensverträgen getroffenen Vereinbarungen, teils in Form bilateraler Verträge. Inhaltlich wurden dabei neben den für alle Staatsbürger geltenden Garantien von Freiheitsrechten wie der Schutz des Lebens, der persönlichen Freiheit sowie Glaubens-, Religions-, und Bekenntnisfreiheit - besondere Regelungen für Minderheiten getroffen. Es wurden das umfassende Diskriminierungsverbot hinsichtlich Rasse, Religion und Sprache sowie Regelungen zu Spracherleichterungen vor Gericht und für das Unterrichtswesen normiert und auch finanzielle Unterstützung für kulturelle Aktivitäten gewährt. ${ }^{18}$ Außerdem wurden Regelungen zu Fragen der Staatsangehörigkeit getroffen. ${ }^{19}$

Die meisten dieser völkerrechtlichen Verpflichtungen sind nach dem Zweiten Weltkrieg untergegangen. Eine Studie des UN-Generalsekretariats legt dazu im Einzelnen die verschiedenen Gründe dar. ${ }^{20}$ Dazu zählen der Untergang des Völkerbundes, der Wegfall einiger Völkerrechtssubjekte sowie eine grundlegende Änderung der Umstände (clausula rebus sic stantibus). Außerdem verlor der Hauptprotagonist der Minderheitenrechte, das Deutsche Reich, sein moralisches Ansehen in der internationalen Staatengemeinschaft. Mit der zunehmenden ideologischen Einfärbung im weiteren Kontext des Ost-West-Konflikts, bei dem sich die Sowjetunion und Jugoslawien als starke Befürworter des Minderheitenschutzes dem ,Westen“ gegenübergestellt sahen, verloren kollektive Minderheitenrechte schließlich bis in die Mitte des 20. Jahrhunderts zunehmend an Bedeutung. Der Westen, und hierbei insbesondere die USA, sowie auch andere auf nationale In-

\footnotetext{
${ }^{16}$ Felix Ermacora, Historische Entwicklung der Menschenrechte und Grundfreiheiten, 1974, S. 350, \147.

17 Ebd.

18 Ebd., S. 353, \150.

${ }^{19}$ Scherer-Leydecker, Minderheiten und sonstige ethnische Gruppen, S. 39-40; Ermacora, Historische Entwicklung der Menschenrecbte und Grundfreibeiten, S. 353, \150.

${ }^{20}$ Commission on Human Rights, ,,Study of the Legal Validity of the Undertaking concerning Minorities“, UN Doc. E/CN.4/367, 7. April 1950; Doehring Karl, „Das Gutachten des Generalsekretärs der Vereinten Nationen über die Fortgeltung der nach dem ersten Weltkrieg eingegangenen Minderheitenschutzverpflichtungen“" Zeitschrift für ausländisches öffentliches Recht und Völkerrecht, Vol. 15, 1953, S. 521.
} 
tegration bedachte außereuropäische Staaten wie Indien und China verfolgten die Konzepte des melting pot und des nation building. ${ }^{21}$ Das favorisierte System von individuellen, allgemeinen und universellen Menschenrechten wurde als ausreichend angesehen, kulturellen und religiösen Minderheiten ihre Freiheiten garantieren zu können. Kollektive Rechte, die unterschiedlichen Gruppen im Staat oder Einwanderern eine gemeinsame Basis gegeben hätten, wurden aus politischen Gründen staatlicher Einheit hingegen abgelehnt.

Entgegen der Politiken der Integration und der überwiegenden Bedeutung der individuellen Menschenrechte konnte die Sowjetunion einen Minderheitenschutzartikel im System der internationalen Menschenrechte verankern. Dabei wurde schwer um einen Kompromiss in der Formulierung gerungen, die eine individualrechtliche Ausgestaltung für Mitglieder einer Minderheit beschreibt und gleichzeitig die kulturelle Integrität der Gruppe der Minderheit schützt. ${ }^{22}$ So fand 1966 Artikel 27 zum Schutz von ethnischen, religiösen und sprachlichen Minderheiten Einzug in den rechtlich verbindlichen Internationalen Pakt für bürgerliche und politische Rechte.

Seit dem Fall der Mauer und den darauf folgenden Bewegungen in den Staaten des ehemaligen Ostblocks haben ethnische Konflikte und die damit einhergehenden Fragen des Minderheitenschutzes wieder an trauriger Brisanz gewonnen. Der Krieg im ehemaligen Jugoslawien und die Konflikte unter der Beteiligung Russlands in Südossetien, Abchasien und der Ukraine, bei denen ethnische Zugehörigkeiten zumindest in der Begründungsrhetorik eine Rolle spielen, stehen dabei nur stellvertretend für die gewaltsamen Auseinandersetzungen der jüngeren Zeit. Aber auch nicht-militärische Spannungen im Kontext des Minderheitenschutzes finden sich aktuell in den Medien, wie etwa das Beispiel der Politik der Rückführung der Roma von Frankreich nach Rumänien zeigt. ${ }^{23}$

\section{Rechtsquellen des internationalen Minderheitenschutzes}

Der Schutz ethnischer und nationaler Minderheiten findet sich in einer Vielzahl von internationalen Abkommen, Konventionen und Deklarationen. Die verschiedenen Instrumente mit Regelungen zu Minderheiten lassen sich grob in vier Entwicklungsstränge einteilen, die sich aus ihren historischen Kontexten ableiten: die historischen Verträge seit dem 16. Jahrhundert, Instrumente zur Zeit des Völkerbundes, die im Kontext der individualrechtlichen Schutzmechanismen entstandenen Entwicklungen im Rahmen der Vereinten Nationen sowie

\footnotetext{
${ }^{21}$ Scherer-Leydecker, Minderheiten und sonstige ethnische Gruppen, S. 43.

22 Nicola Wenzel, Das Spannungsverhältnis zwischen Gruppenschutz und Individualschutz im Völkerrecht, 2008.

23 „France sends Roma back to Romania“, BBC, 20. August 2010.
} 
moderne Entwicklungen, die wiederum dem kollektivrechtlichen Charakter von Schutzmechanismen mehr Bedeutung zumessen. ${ }^{24}$

\section{Historische Rechtsquellen}

Die bereits genannten historischen Dokumente des Augsburger Religionsfriedens (1555), des Westfälischen Friedens (1648) und des Wiener Kongresses (1815) sowie eine Reihe von bilateralen Abkommen dieser Zeit beinhalten vereinzelte Regelungen zum Schutz von Religionsgruppen und bilden damit die Anfänge des internationalen Minderheitenschutzes. ${ }^{25}$ Die Verträge von Paris (1856) ${ }^{26}$ und Berlin (1878) ${ }^{27}$ bezogen sich bereits explizit auf religiöse Minderheiten. Diese historischen Dokumente bilden somit die konzeptionellen Vorläufer des internationalen Minderheitenschutzes.

\section{Rechtsquellen aus der Zeit des Völkerbundes}

Die Satzung des Völkerbundes enthält keine ausdrücklichen Regelungen zum Schutz von Minderheiten, da die Siegermächte eine Bindung aller Mitglieder, also einschließlich ihrer selbst, nicht akzeptieren wollten. ${ }^{28}$ Allerdings wurde eine Reihe von Verträgen zwischen den Alliierten, assoziierten Hauptmächten und einzelnen Staaten geschlossen, die jeweils individuelle Schutzvorschriften enthielten. ${ }^{29}$ Für Österreich, Bulgarien, Ungarn und die Türkei wurden Regelungen bereits im Rahmen der Friedensverträge getroffen. ${ }^{30}$ Polen, die Tschechoslowakei, das Königreich der Serben, Kroaten und Slowenen, Rumänien und Griechenland gingen entsprechende Verträge in den Jahren 1919 und 1920 ein. ${ }^{31}$ Daneben wurden weitere bilaterale Verträge geschlossen, beispielsweise zum Schutz der polnischen Minderheit in der Freien Stadt Danzig oder zwischen Finnland und Schweden hinsichtlich der schwedischen Minderheit auf den

\footnotetext{
${ }^{24}$ Natan Lerner, „The Evolution of Minority Rights in International Law“, in: Catherine Brölmann, René Lefeber, und Marjoleine Zieck (Hrsg.), Peoples and Minorities in International Law, 1993, S. 81.

${ }^{25}$ Peter Pernthaler, „Zur Entstehung des völkerrechtlichen Menschenrechts- und Minderheitenschutzes im 19. und 20. Jahrhundert", in: Christoph Pan und Beate Sibylle Pfeil (Hrsg.), Handbuch der europäischen Volksgruppen, 2000, S. 16-20.

${ }^{26}$ „Traité de paix signé à Paris le 30 mars 1856 entre la Sardaigne, l'Autriche, la France, le Royaume Uni de la Grande Bretagne et d'Irlande, la Prusse, la Russie et la Turquie“, 114 CTS, 409, 30. März 1856, Art. 9.

27 „Vertrag zwischen Deutschland, Österreich-Ungarn, Frankreich, Großbritannien, Italien, Rußland und der Türkei. (Berliner Vertrag)“, Deutsches Reichsgesetzblatt, Band 1878, Nr. 31, S. 307-345, 13. Juli 1878, Art. 5, 15, 27, 35, 44, 62.

${ }^{28}$ Scherer-Leydecker, Minderheiten und sonstige ethnische Gruppen, S. 33.

${ }^{29}$ Jacob Robinson, „International Protection of Minorities a global view“, Israel Yearbook on Human Rights, Vol. 1, Nr. 1, 1971, S. 63f., 74.

${ }^{30}$ Ebd., S. 63f.

${ }^{31}$ Ebd., S. 63.
} 
Ålandinseln. ${ }^{32}$ Außerdem wurde eine Vielzahl von unilateralen Erklärungen abgegeben. ${ }^{33}$ Der Ständige Internationale Gerichtshof fasste die Grundidee der unterschiedlichen Regelungen dieser Instrumente in seiner Entscheidung Minority Schools in Albania zusammen: Es gehe um die Sicherstellung der Möglichkeit, dass Minderheiten in Frieden und in freundschaftlicher Beziehung zur Bevölkerung eines Staates leben und dabei ihre Eigenheiten bewahren können. ${ }^{34}$

Die Geschichte des Zweiten Weltkrieges, die nicht nur zur Auflösung des Völkerbunds führte, bedeutete eine grundsätzliche Veränderung der zwischenstaatlichen Ordnung Europas. ${ }^{35}$ Durch den Wegfall einiger Völkerrechtssubjekte und die grundsätzliche Veränderung der Umstände (clausula rebus sic stantibus) gelten die Minderheitenschutzgarantien aus der Zeit des Völkerbundes bis auf die Verträge zu den Ålandinseln und den Minderheiten in der Türkei und Griechenland als nach 1946 erloschen. ${ }^{36}$

\section{Minderheitenschutzinstrumente im modernen Völkerrecht}

Mit den Vereinten Nationen entwickelte sich nach dem Zweiten Weltkrieg das System des internationalen Menschenrechtsschutzes, welches mit seinem Anspruch des individualrechtlichen Diskriminierungsverbots den kollektivrechtlichen Minderheitenschutz verdrängte. ${ }^{37}$ Trotz Bekräftigung der Notwendigkeit sich mit dem Schicksal von Minderheiten zu beschäftigen, wurden im Zuge der Verhandlungen zur Allgemeinen Erklärung der Menschenrechte die Vorschläge abgelehnt, eine entsprechende Regelung aufzunehmen. ${ }^{38}$ Allerdings leisteten Dokumente der 1946 eingerichteten UN Sub-commission on the Prevention of Discrimination and Protection of Minorities Vorarbeiten für Artikel 27 des 1966 verabschiedeten Internationalen Pakts für bürgerliche und politische Rechte. ${ }^{39}$ Artikel 27 IPbpR bildet mit seiner globalen und rechtsverbindlichen Anwendbarkeit für den internationalen

\footnotetext{
${ }^{32}$ Lerner, „The Evolution of Minority Rights in International Law“, S. 83; Scherer-Leydecker, Minderbeiten und sonstige etbnische Gruppen, S. 35; Eine Liste der Verträge findet sich bei: Thornberry, International law and the rights of minorities, S. $41 \mathrm{f}$.

33 Thornberry, International law and the rights of minorities, S. $41 \mathrm{f}$.

${ }^{34}$ Permanent Court of International Justice, „Minority Schools in Albania“, 1935 PCIJ, Ser. A/B, No. 64, 6. April 1935, S. 17.

35 Siehe dazu „A. I. Geschichte des Minderheitenschutzes im Völkerrecht“ auf S. 14.

${ }^{36}$ Commission on Human Rights, ,Study of the Legal Validity of the Undertaking concerning Minorities", UN Doc. E/CN.4/367, S. 70.

${ }^{37}$ Lerner, „The Evolution of Minority Rights in International Law“, S. 87; Natan Lerner, Group rights and discrimination in international law, 1991, S. 14.

38 UN Generalversammlung, „International Bill of Human Rights“, UN Doc. A/Res/3/217(III)C, 10. Dezember 1948, Abschnitt C, „Fate of Minorities“; Lerner, „The Evolution of Minority Rights in International Law", S. 87.

39 Asbjørn Eide, „Historical and Global Perspectives“, in: Marc Weller (Hrsg.), The Rights of Minorities: A Commentary on the European Framework Convention for the Protection of National Minorities, 2005, S. $40 \mathrm{f}$.
} 
Minderheitenschutz der Gegenwart die wichtigste Rechtsquelle. Sie ist die Grundlage für Entwicklungen in der UN Menschenrechtskommission und die daraus hervorgegangenen Berichte der Sonderberichterstatter Francesco Capotorti und Asbjørn Eide. ${ }^{40}$ Diese Arbeiten machten die Notwendigkeit einer eigenen Minderheitendeklaration deutlich, welche schließlich zur Declaration on the Rights of Persons Belonging to National or Ethnic, Religious and Linguistic Minorities führte, die 1992 von der UN Generalversammlung ohne Abstimmung angenommenen wurde. ${ }^{41}$ Unter dem Dach der Vereinten Nationen finden sich heute der Human Rights Council, der Independent Expert on Minority Issues sowie das Forum on Minority Issues, die durch Berichtsverfahren und Expertisen den globalen Minderheitenschutz weiterentwickeln. ${ }^{42}$ Schließlich entwickelten sich in Hinblick auf Artikel 27 IPbpR im Rahmen des Staaten- und Individualbeschwerdeverfahrens vor dem Menschenrechtsausschuss weitere Konkretisierungen des Minderheitenschutzes. ${ }^{43}$

\section{Neuere Entwicklungen im Sinne einer kollektivrechtlichen Ausgestaltung}

Im Zuge der Auflösung der Sowjetunion und der Neuordnung ihrer Satellitenstaaten gewannen Fragen des internationalen Minderheitenschutzes seit den 1990er Jahren wieder zunehmend an Bedeutung, wobei sich im Zuge dieses aufkommenden Interesses in der Literatur wieder eine vornehmlich kollektivrechtliche Perspektive herausbildete. Diese kollektivrechtliche Lesart von Dokumenten und Quellen konstituiert die vierte Entwicklungslinie des internationalen Minderheitenschutzes seit den historischen Verträgen ab dem 16. Jahrhundert, den Instrumenten aus der Zeit des Völkerbundes und den individualrechtlichen Schutzmechanismen aus dem Kontext der Vereinten Nationen. ${ }^{44}$

\footnotetext{
40 Sub-Commission on the Prevention of Discrimination and Protection of Minorities, Francesco Capotorti, ,Study on the rights of persons belonging to ethnic, religious, and linguistic minorities“, UN Doc. E/CN.4/Sub.2/384/Rev.1, 1979; Sub-Commission on the Prevention of Discrimination and Protection of Minorities, Asbjørn Eide, „Preliminary Report submitted by the Special Rapporteur Mr. Asbjørn Eide, on possible ways and means of facilitating the peaceful and constructive solution of problems involving minorities", UN Doc. E/CN.4/Sub.2/1990/46, 20. Juli 1990.

${ }^{41}$ UN Generalversammlung, ,Declaration on the Rights of Persons Belonging to National or Ethnic, Religious and Linguistic Minorities“, UN Doc. A/Res/47/135, 18. Dezember 1992.

$42 \mathrm{Zu}$ den Arbeiten im Kontext des Human Rights Councils, dem Forum for Minority Issues und dem Independent Expert siehe „A. IV. 1. UN Institutionen“ auf S. 30.

${ }^{43} \mathrm{Zu}$ den Institutionen der Berichtsverfahren und Durchsetzungsmechanismen siehe „A. IV. 1. UN Institutionen“ auf S. 30 sowie „A. IV. 2. Berichts- und Beschwerdemechanismen unter dem IPbpR" auf S. 33.

44 Wenzel, Das Spannungsverhältnis zwischen Gruppenscbutz und Individualschutz im Völkerrecht, S. 16 und 20; Manfred Nowak, „The Evolution of Minority Rights in International Law, Comments“, in: Catherine Brölmann, René Lefeber, und Marjoleine Zieck (Hrsg.), Peoples and Minorities in International Law, 1993, S. 108; Eibe Riedel, „Gruppenrechte und kollektive Aspekte individueller Men-
} 
A. Der internationale Minderheitenschutz

Die rechtswissenschaftliche Literatur interpretiert heute beispielsweise die im Zuge der Nachwirkungen des Zweiten Weltkriegs von der UN Generalversammlung angenommene Konvention über die Verbütung und Bestrafung des Völkermords (1948) als kollektivrechtliches Instrument. ${ }^{45}$ Im Unterschied zu anderen Menschenrechtsinstrumenten dieser Zeit liegt ihr Fokus nicht auf Individualrechten, sondern folgt einem kollektivrechtlichen Ansatz zum Schutz von verfolgten nationalen, ethnischen, rassischen und religiösen Gruppen. Auch die Internationale Konvention über die Beseitigung jeder Form der Rassendiskriminierung $(1965)^{46}$, die 1978 von der Generalkonferenz der UNESCO angenommene Erklärung über Rassen und rassistische Vorurteile ${ }^{47}$ und die bereits genannte UN Declaration on the Rights of Persons Belonging to National or Ethnic, Religious and Linguistic Minorities von 1992 begreifen den Minderheitenschutz in einer gruppenrechtlichen Ausgestaltung. 48

Auf regionaler Ebene zeichneten sich die vergleichsweise intensivsten Entwicklungen des Minderheitenschutzes im europäischen Raum ab. Im Rahmen der Organisation für Sicherheit und Zusammenarbeit in Europa (OSZE) wurden unterschiedliche normative Dokumente verabschiedet sowie ein Hochkommissariat für nationale Minderheiten eingerichtet. ${ }^{49}$ Bereits 1990, in einer Zeit großer politischer Veränderungen, setzte die OSZE mit dem Copenhagen Document einen Meilenstein der normativen Anerkennung von Minderheiten. Sie wandte sich gegen die Politik der Assimilation kulturell distinkter Gruppen und proklamierte deutlich, dass „to belong to a national minority is a matter of a person's individual choice and no disadvantage may arise from the exercise of such choice“. ${ }^{0}$ Auch in der Europäi-

schenrechte“, in: Walter Kälin u.a. (Hrsg.), Aktuelle Probleme des Menschenrechtsschutzes, Berichte der Deutschen Gesellschaft für Völkerrecht 33, 1994, S. 49.

${ }^{45}$ Convention on the Prevention and Punishment of the Crime of Genocide (Übereinkommen über die Verhütung und Bestrafung des Völkermordes), 78 UNTS 277, 9. Dezember 1948, s. insbesondere Art. 2.

46 International Convention on the Elimination of All Forms of Racial Discrimination (Internationales Übereinkommen zur Beseitigung jeder Form von Rassendiskriminierung), 660 UNTS 195, 7. März 1966, s. insbesondere Art. 2 Abs. 1 und 2, der Gruppen als solche schützt, allerdings ohne diese als Rechtsträger anzuerkennen.

${ }^{47}$ UNESCO, „Declaration on Race and Racial Prejudice“, UN Doc. E/CN.4/Sub.2/1982/2/Add.1, Annex V, 27. November 1978, s. insbesondere Art. 1 Abs. 2 und Art. 5 Abs. 1.

48 Wenzel, Das Spannungsverbältnis zwischen Gruppenschutz und Individualschutz im Völkerrecht, S. 42f; UN Generalversammlung, „Declaration on the Rights of Persons Belonging to National or Ethnic, Religious and Linguistic Minorities“, UN Doc. A/Res/47/135.

49 Eine Übersicht der Dokumente ist zusammengestellt bei Björn Arp, International norms and standards for the protection of national minorities, 2008, S. 169-190; Jakob Haselhuber, „Institutionalisierung ohne Verrechtlichung: der Hohe Kommissar für nationale Minderheiten der OSZE“, in: HansJoachim Heintze (Hrsg.), Moderner Minderbeitenschut:: rechtliche oder politische Absicherung?; Zum 50. Jahrestag der UN-Menschenrechtserklärung, 1998, S. 116-137.

${ }^{50}$ Organisation for Security and Co-operation in Europe (OSZE, vormals Conference on Security and Co-operation in Europe, CSCE), ,Document of the Copenhagen Meeting of the Confer- 
schen Union wurden zahlreiche Dokumente zu Minderheitenfragen verabschiedet. Die Achtung und der Schutz von Minderheiten wurden im Rahmen der Kopenhagen Kriterien explizit zur Voraussetzung für einen Beitritt erklärt..$^{51} 1992$ erarbeitete der Europarat die Europäische Charta der Regional- und Minderheitensprachen sowie 1995 die Rabmenkonvention zum Schutz nationaler Minderbeiten.52 Des Weiteren wurden insbesondere seit 1990 in Zentral- und Osteuropa circa 200 bilaterale Verträge zum Schutz von Minderheiten geschlossen. ${ }^{53}$

In einer Gesamtschau dieser Vielzahl von Rechtsquellen lässt sich feststellen, dass die modernen multilateralen Minderheitenschutzinstrumente einen rechtlich unverbindlichen und nicht hinreichend konkretisierten Gehalt an Verpflichtungen aufweisen und somit nur als soft-law einzuordnen sind. ${ }^{54}$ Insbesondere die UN Deklaration (1992), die Dokumente der OSZE und des Europarats sind sprachlich offen gestaltet und lassen einen weiten Interpretationsspielraum für die darin niedergelegten Regelungen zu. ${ }^{55}$ Im Gegensatz dazu bildet Artikel 27 IPbpR eine rechtsverbindliche Grundlage globaler Minderheitenschutzgarantien, die sich im Rahmen der internationalen Berichts- und Beschwerdemechanismen zunehmend konkretisiert hat.

III. Inhalt des internationalen Minderheitenschutzes

Artikel 27 IPbpR normiert in nahezu universeller Anwendbarkeit das Minimum des Minderheitenschutzniveaus, welches - wenn auch nur teilweise - weitergehend durch regionale und bilaterale Verträge ergänzt wird. Die internationalen Minderheitenschutzrechte verpflichten Staaten, den Mitgliedern von Minderheiten das gemeinsame (Er-)leben der Kultur, die gemeinsame Ausübung der Religion und gemeinsame Nutzung der Sprache nicht vorzuenthalten. Diese Verpflichtung geht dabei über ein Diskriminierungsverbot hinaus und soll durch aktive Schutzmaßnahmen des Staates gegen Diskriminierung und durch eine privilegierte Behandlung eine wirkliche Gleichbehandlung ermöglichen. ${ }^{56}$ In der

ence on the Human Dimension of the CSCE“, 29. Juni 1990, Abs. 32; Rianne Monique

Letschert, The impact of minority rights mechanisms, 2005, S. 13-16.

${ }^{51}$ Ein Überblick zu den Entwicklungen in der EU findet sich bei Arp, International norms and standards for the protection of national minorities, S. 190-216; Kristin Henrard, „,The Impact of the Enlargement Process on the Devleopment of a Minority Protection Policy within the EU; Another Aspect of Responsibility/Burden-Sharing?", Maastricht Journal of European and Comparative law, Vol. 9, Nr. 4 (2002), S. 357-393.

52 European Charter for Regional or Minority Languages (Europäische Charta der Regional- und Minderheitensprachen), CETS 148, 5. November 1992; Framework Convention for the Protection Of National Minorities (Rahmenübereinkommen zum Schutz nationaler Minderheiten, FCNM), 2151 UNTS 243, 1. Februar 1995.

53 Arp, International norms and standards for the protection of national minorities, S. XXIX.

${ }^{54}$ Letschert, The impact of minority rights mechanisms, S. 27.

55 Ebd.

${ }^{56}$ Manfred Nowak, U.N. covenant on civil and political rights, 2005, Art. 27, Rn. 40. 
A. Der internationale Minderheitenschutz

Literatur haben Untersuchungen zum Schutzumfang von Minderheitenrechten eine Ausdifferenzierung erfahren, welche die expliziten und impliziten sowie die bestehenden und als notwendig erachteten Schutzgehalte im Detail verdeutlicht. ${ }^{57}$ So kann man zwischen dem Recht auf physische Existenz und Integrität, dem Recht auf Identität, dem Recht auf Heimat, dem Recht auf Nichtdiskriminierung und positive Diskriminierung, dem Namensrecht, dem Sprachenrecht, dem Recht auf Minderheitenreligion bzw. -kultur und deren Förderung, dem Recht auf grenzüberschreitenden Kontakt, Schulrechten, dem Recht auf Partizipation und Repräsentation, Autonomierechten und Rechten auf Dezentralisierung und schließlich dem Recht auf Verhandlungen über den Minderheitenstatus unterscheiden. ${ }^{58}$

Um ein konkreteres Bild der Grundzüge des Schutzgehaltes zu zeichnen, sollen im Folgenden die drei grundlegenden Säulen des Schutzbereichs - dies sind namentlich die Pflege des kulturellen Lebens, das Bekennen und Ausüben der eigenen Religion und das Bedienen der eigenen Sprache -, wie sie so auch in Artikel $27 \mathrm{IPbpR}$ genannt sind, näher dargestellt werden.

\section{Das Recht auf Kultur}

Das Recht auf Kultur und zur Pflege des kulturellen Lebens umfasst nicht nur Gebräuche, Sitten, Traditionen, Rituale, Arten des Wohnens, Essgewohnheiten usw., sondern schließt auch ökonomische Handlungsweisen mit ein. So wurde beispielsweise anerkannt, dass Artikel 27 IPbpR auch die Rentier- und Rinderzucht sowie das Jagen und Fischen umfasst. Des Weiteren werden Kunstgewerbe, das Spielen von Musik sowie das Betreiben von Kulturbetrieben und Verlagen für Veröffentlichungen in der eigenen Sprache geschützt. ${ }^{59}$ Der Begriff des kulturellen Lebens ist dabei weit gefasst. ${ }^{60}$ Es geht nicht um eine ,Musealisierung $^{6}$ von Kultur, denn dies würde dem ständigen Prozess der Veränderung von Kultur widersprechen. Vielmehr soll ein rechtlich geschützter Raum geschaffen werden, in dem das kulturelle Leben gepflegt und weiterentwickelt werden kann. ${ }^{61}$

\footnotetext{
${ }^{57}$ Eine ausdifferenzierte Darstellung der von ihm als notwendig erachteten Schutzgehalte findet sich bei Ralf Roßkopf, Theorie des Selbstbestimmungsrechts und Minderbeitenrechts, Abhandlungen zu Migration und Flüchtlingsfragen, 2004, S. 474-505.

${ }^{58}$ Ebd., S. XIX-XXI.

${ }^{59}$ Nowak, U.N. covenant on civil and political rights, Art. 27, Rn. 41.

${ }^{60}$ Sub-Commission on the Prevention of Discrimination and Protection of Minorities, Francesco Capotorti, ,Study on the rights of persons belonging to ethnic, religious, and linguistic minorities“, UN Doc. E/CN.4/Sub.2/384/Rev.1, Abs. 327ff.

${ }^{61}$ Patrick Thornberry, ,The UN Declaration on the Rights of Persons Belonging to National or Ethnic, Religious and Linguistic Minorities: Background, Analysis, Observations, and an Update“, in: Alan Phillips und Allan Rosas (Hrsg.), Universal Minority Rights, 1995, S. 21.
} 
Grundlage des kulturellen Lebens ist der Lebensraum, so dass der Schutzumfang von Minderheitenrechten auch den Schutz vor Umsiedlung umfasst. Das Advisory Committee der Framework Convention for the Protection of National Minorities hat vor dem Hintergrund des EGMR Falles Noack and Others v. Germany in seiner Opinion festgestellt, dass „die Auflösung von Gemeinden, in denen Mitglieder von nationalen Minderheiten traditionell leben, unwiderlegbar nahelegt, dass der Erhalt ihrer Identität erschwert wird." 62 Dabei ging es um die Umsiedlung des sorbischen Dorfes Horno in Brandenburg, welches dem Braunkohletagebau weichen musste. Das Gericht stellte fest, dass „,for the purposes of Article 8 of the [European] Convention [of Human Rights], a minority's way of life is, in principle, entitled to the protection guaranteed for an individual's private life, family life and home." 63 Der Antrag wurde zwar mangels nationaler Rechtswegerschöpfung und prima facie Unbegründetheit als unzulässig abgewiesen, da eine Umsiedlung in ein 20 Kilometer entferntes Dorf nicht unverhältnismäßig gewesen sei. ${ }^{64}$ Das Advisory Committee der Framework Convention mahnte hingegen an, dass bei solchen Entscheidungen in Zukunft Artikel 5 der Framework Convention in die Abwägung zwischen dem Schutz der Kultur und dem Erhalt der Identität der Minderheit auf der einen Seite und dem öffentlichen Interesse auf der anderen mit einzubeziehen sei. ${ }^{65}$

Zum Recht zur Pflege der Kultur stellt das Human Rights Committee in seinem general comment fest, ,that culture manifests itself in many forms, including a particular way of life associated with the use of land resources". ${ }^{66}$ Unter dem unbestimmten Begriff ,Kultur' hat sich im Zuge von Entscheidungen und Kommentaren somit ein konkreter Schutzumfang für kulturelle Praxen, Gebräuche und Sitten bis hin zu Land-, und Jagdrechten etabliert.

\section{Religionsfreibeit}

Auch wenn der Schutz der Religionsfreiheit menschenrechtlich garantiert ist, erlangt der Schutz des Bekenntnisses und Ausübens der eigenen Religion im Kontext des Minderheitenschutzes eine umfassendere Ausgestaltung. Aus der Systematik der Artikel 27 sowie 18, 19, 21 und 22 IPbpR ergibt sich mangels

${ }^{62}$ Advisory Committee on the Framework Convention for the Protection of National Minorities, „Opinion on Germany“, ACFC/INF/OP/I (2002) 008, 1. März 2002, Abs. 32 (meine Übersetzung); Europäischer Gerichtshof für Menschenrechte, „Noack and Others v. Germany (46346/99)“, Reports of Judgments and Decisions / Recueil des Arrêts et Décisions, 2000-VI, 25. Mai 2000; Geoff Gilbert, „Article 5“, in: Marc Weller (Hrsg.), The Rights of Minorities: A Commentary on the European Framework Convention for the Protection of National Minorities, 2005, S. $166 \mathrm{ff}$.

${ }^{63}$ EGMR, „Noack and Others v. Germany (46346/99)“, Abs. 550.

${ }^{64}$ Ebd., Abs. 553.

65 Advisory Committee on the Framework Convention for the Protection of National Minorities, „Opinion on Germany“, ACFC/INF/OP/I (2002) 008, Abs. 32.

${ }^{66}$ Human Rights Committee, „General Comment No. 23: The rights of minorities (Art. 27)“, CCPR/C/21/Rev.1/Add.5, 8. April 1994, Abs. 7. 
A. Der internationale Minderheitenschutz

einer expliziten Schrankenregelung im Minderheitenschutz ein umfänglicherer Schutz der Religionsfreiheit für Minderheiten, der nur in Abwägung mit anderen Menschenrechten, nicht aber durch einfache Gesetze einzuschränken ist. ${ }^{67}$ Zwar bestand während der Verhandlungen ein Verständnis der Religionsfreiheit des Artikels $27 \mathrm{IPbpR}$ parallel zu dem des Artikels $18 \mathrm{IPbpR}$, was somit auch die Schranken aus Artikel 18 (3) IPbpR implizieren würde, ${ }^{68}$ allerdings verbietet sich solch eine Auslegung unter Rückgriff auf die travaux préparatoires wegen des eindeutigen Wortlauts. ${ }^{69}$ Schließlich gebietet der Sinn und Zweck des Minderheitenschutzes einen umfänglicheren Schutz der Religion von Minderheiten. ${ }^{70}$ Der Schutz der Religionsfreiheit im Rahmen des Minderheitenschutzes, wie er explizit auch in Artikel 8 der Framework Convention niedergelegt wurde, ${ }^{71}$ umfasst demnach zumindest die Freiheit des Bekenntnisses und der Ausübung der Religion in Form des Forum Internum und Forum Externum und besteht ergänzend zur individuellen, menschenrechtlich garantierten Religionsfreiheit.

\section{Das Recht zum Gebrauch der eigenen Sprache}

Das Recht zur Nutzung der gemeinsamen Sprache umfasst das geschriebene und gesprochene Wort in der Öffentlichkeit und im Privaten. Staaten dürfen weder die Nutzung der Sprache in der Öffentlichkeit, noch die Veröffentlichung von Literatur oder das Erlernen der Sprache für Kinder verbieten. ${ }^{72}$ Der gemeinsamen Sprache kommt im Minderheitenschutz eine besondere Bedeutung zu, da sie eine zentrale Rolle im Erhalt der eigenen Kultur spielt und als identitätsstiftendes Element im täglichen Leben präsent ist. Minderheitensprachen können dazu auch eine rechtliche Privilegierung erfahren, die wiederum andere Menschenrechte einschränken kann. Eine Ausgestaltung solcher staatlicher Regelungen zur Nutzung bestimmter Sprachen im öffentlichen Raum wurde im Fall Ballantyne et al. v. Canada vor dem Human Rights Committee in Abwägung gegenüber der Meinungsfreiheit konkretisiert. ${ }^{73}$ Danach ist eine Einschränkung der Meinungsfreiheit durch Regelungen zum Minderheitenschutz - hier konkret das

\footnotetext{
${ }^{67}$ Nowak, U.N. covenant on civil and political rights, Art. 27, Rn. 53.

${ }^{68}$ Christian Tomuschat, „Protection of Minorities under Article 27 of the International Covenant on Civil and Political Rights“, in: Rudolf Bernhardt u.a. (Hrsg.), Völkerrecht als Rechtsordnung, internationale Gerichtsbarkeit, Menschenrechte: Festscbrift für Hermann Mosler, Beiträge zum ausländischen öffentlichen Recht und Völkerrecht, Band 81, 1983, S. 976.

${ }^{69}$ Nowak, U.N. covenant on civil and political rights, Art. 27, Rn. 53.

${ }^{70}$ Ebd.

${ }^{71}$ Ausführlich dazu: Zdenka Machnyikova, „Article 8“, in: Marc Weller (Hrsg.), The rights of minorities in Europe, 2006, S. 225.

${ }^{72}$ Nowak, U.N. covenant on civil and political rights, Art. 27, Rn. 42.

73 UN Generalversammlung, „Report of the Human Rights Committee“, Communication Nos. 359/1989 and 385/1989, John Ballantyne and Elizabeth Davidson, and Gordon McIntyre v. Canada (Ballantyne et al. v. Canada), UN Doc. A/48/40(PARTII), 1. November 1993, S. 91.
} 
Aufhängen englischer Werbung im französischsprachigen Quebec - zwar grundsätzlich möglich, im konkreten Fall sei eine solche Einschränkung zum Schutz der frankophonen Minderheit allerdings nicht notwendig gewesen. ${ }^{74}$ Anderer Ansicht war Ndiaye in seinem Sondervotum, der die Einschränkung der Meinungsfreiheit durch die Minderheitenschutzregelung als gerechtfertigt ansah. ${ }^{75}$

Es wird kontrovers diskutiert, inwieweit Staaten verpflichtet sind, Minderheitensprachen als Amtssprachen zu führen, um Mitgliedern von Minderheiten die Möglichkeit zu geben, in ihrer Sprache mit Behörden und vor Gericht kommunizieren zu können. In Diergaardt et al. v. Namibia vermied das Human Rights Committee in dieser Frage die direkte Anwendung von Artikel 27 IPbpR und beurteilte das Verbot der Nutzung von Afrikaans durch die namibischen Behörden unter dem Gebot der Nichtdiskriminierung: Afrikaans sei gleich anderen Stammessprachen zu behandeln. ${ }^{76}$ In einem der sechs Sondervoten stellte Fajsoomer Lallah fest, Artikel 27 IPbpR würde zu weit ausgelegt werden, hätte das Committee vorgeschlagen, dass eine nicht-offizielle Sprache von den Behörden zu nutzen sei.

Artikel 10 (2) der Framework Convention for the Protection of National Minorities normiert eine entsprechende Forderung. Die Staaten werden dazu angeregt in Regionen, in denen Minderheiten traditionell und auch in substantieller Anzahl leben, auf Anfrage der Minderheit ihnen die Nutzung ihrer Sprache im behördlichen Verkehr zu ermöglichen. ${ }^{77}$ Artikel 10 (3) der Framework Convention regelt weiterhin, wie dies auch aus dem menschenrechtlichen Gebot des fair trial hervorgeht, dass in Strafsachen zumindest ein Dolmetscher bereitgestellt werden muss. ${ }^{78}$ Der Schutz der Sprache gilt als wichtigstes Element des Minderheitenschutzes. Er wird in den normativen Quellen umfassend formuliert, so dass sich daraus sogar das Erfordernis aktiver staatlicher Maßnahmen ergeben kann.

\section{Umsetzung des internationalen Minderheitenschutzes}

Der Minderheitenschutz im Kontext der internationalen Menschenrechte, so wie er sich in Artikel 27 IPbpR manifestiert, garantiert einen ungehinderten Zugang und privilegierten Schutz der Pflege des kulturellen Lebens, das Bekennen und Ausüben der eigenen Religion und das Sprechen der eigenen Sprache. Die neueren Dokumente erweitern diesen Schutzumfang: Die UN Minderhei-

\footnotetext{
${ }^{74}$ Ebd., S. 103, Abs. 11.4.

75 Ebd., S. 105 f.

${ }^{76}$ UN Generalversammlung, „Report of the Human Rights Committee“, Communication No. 760/1997, J.G.A. Diergaardt et al. v. Namibia, UN Doc. A/55/40[Vol.II](SUPP), 18. Oktober 2000, Annex IX. M., S. 140, 147, Abs. 10.10 und 11.

${ }^{77}$ Fernand de Varennes, „Article 10“, in: Marc Weller (Hrsg.), The rights of minorities in Europe, 2006, S. 309 .

${ }^{78}$ Ebd., S. 323ff.; Nowak, U.N. covenant on civil and political rights, Art. 27, Rn. 42; Tomuschat, „Protection of Minorities under Article 27 of the International Covenant on Civil and Political Rights", S. 974.
} 
A. Der internationale Minderheitenschutz

tendeklaration etabliert in ihrem Artikel 2 (2) ein umfassendes Recht auf Teilhabe am öffentlichen und insbesondere politischen Leben; ${ }^{79}$ die Framework Convention for the Protection of National Minorities differenziert und konkretisiert diese Ansprüche. Das Normprogramm des internationalen Minderheitenschutzes, also die grundlegende Idee des Erhalts und der Förderung der Vielfalt verschiedener Kulturen und Identitäten, wird in einer Vielzahl unterschiedlicher Völkerrechtsquellen in konkrete staatliche Verpflichtungen umgesetzt.

Aus den allgemeinen Regeln des Völkerrechts ergibt sich, dass der Staat - und nicht etwa Private oder internationale Organisationen - als Vertragspartei an die Verpflichtungen zum Minderheitenschutz gebunden und somit für die Umsetzung der völkerrechtlichen Minderheitenschutzgarantien zuständig ist. ${ }^{80} \mathrm{Im}$ Bereich des Minderheitenschutzes ergeben sich verschiedenen Arten der Implementierung, um den jeweiligen Verpflichtungen nachzukommen. Zu unterscheiden sind dabei das Eingriffsverbot, Schutzpflichten bei Maßnahmen Privater sowie aktive Schutz- und Unterstützungsmaßnahmen seitens des Staates, die den Schutz der Minderheit garantieren. ${ }^{81}$

a) Eingriffsverbot

Minderheitenrechte sind als Menschenrechte in ihrer rechtsstaatlichen Funktion grundsätzlich als Abwehrrechte gegen staatliche Eingriffe konzipiert, so dass, wie dies auch der Wortlaut von Artikel 27 IPbpR nahelegt, den Angehörigen von Minderheiten die garantierten Rechte von Seiten des Staates gemeinsam mit anderen Angehörigen ihrer Gruppe ,nicht vorenthalten“ werden dürfen. ${ }^{82}$ Das bedeutet, dass der Staat nicht in die garantierten Rechte, das kulturelle Leben zu pflegen, die eigene Religion zu bekennen und auszuüben oder sich der eigenen Sprache zu bedienen, eingreifen darf. Er darf beispielsweise weder die Gründung von kulturellen Institutionen, Schulen oder Medien, in denen die Minderheitensprache genutzt wird, verbieten noch Mitgliedern der Minderheit den Zugang zu ihrem Territorium verwehren ${ }^{83}$ oder dieses zu kommerziellen Zwecken enteignen. ${ }^{84}$

79 Thornberry, „The UN Declaration on the Rights of Persons Belonging to National or Ethnic, Religious and Linguistic Minorities: Background, Analysis, Observations, and an Update", S. $42 \mathrm{ff}$.

${ }^{80}$ Stephan Hobe, Einführung in das Völkerrecht, 2008, S. 214, 463.

${ }^{81}$ Scherer-Leydecker, Minderheiten und sonstige ethnische Gruppen, S. 249ff.

82 Der Wortlaut ,shall not be denied“ findet sich in dieser Negatitionsformulierung einzig in Art. 27 IPbpR. Nowak, U.N. covenant on civil and political rights, Art. 27, Rn. 39; Scherer-Leydecker, Minderbeiten und sonstige ethnische Gruppen, S. 250.

83 UN Generalversammlung, „Report of the Human Rights Committee“, Communication No. R.6/24, Sandra Lovelace v. Canada (Lovelace v. Canada), UN Doc. A/36/40 (Supp), 29. September 1981, Annex XVIII, S. 173, Abs. 15.

${ }^{84}$ So rügte das Advisory Committee der Framework Convention im Fall Noack et al. v Germany die Umsiedlung eines sorbischen Dorfes, welches dem Braunkohletagebau weichen musste. Adviso- 
b) Schutzpflicht des Staates bei Handlungen Privater

Die Schutzpflicht des Staates für Minderheitenrechte erstreckt sich auch auf Verletzungshandlungen durch Private. ${ }^{85}$ Insbesondere bei interkulturellen Spannungen, seien dies ethnische Konflikte oder Auseinandersetzungen aufgrund von unterschiedlichen Religionszugehörigkeiten, besteht die Gefahr von Übergriffen durch nicht-staatliche Akteure. Dies umfasst auch Konstellationen von wirtschaftlicher Dominanz einer Partei, welche die Rechte von Minderheiten verletzen kann. Das Human Rights Committee führte dazu weiterführend in seinem general comment aus, dass

a State party is under an obligation to ensure that the existence and the exercise of this right are protected against their denial or violation. Positive measures of protection are, therefore, required not only against the acts of the State party itself, whether through its legislative, judicial or administrative authorities, but also against the acts of other persons within the State party. ${ }^{86}$

Auch die Framework Convention verpflichtet zu Schutzmaßnahmen bei Verletzungshandlungen Privater. ${ }^{87}$ Der Staat, als Träger des Gewaltmonopols, hat somit die Aufgabe, die im Minderheitenschutz gewährten Rechtsgüter zu schützen.

Des Weiteren bestehen staatliche Schutzpflichten in Konflikten zwischen den Institutionen der Minderheit und ihren Mitgliedern. ${ }^{8}$ So stellte das Human Rights Committee in Kitok v. Sweden trotz eines im Ergebnis gerechtfertigten Eingriffs in die Berufsfreiheit von Ivan Kitok durch die Nichtzulassung als Rentier-Züchter fest, dass Schweden wegen seiner gesetzlichen Regelungen eine Schutzpflicht trifft. ${ }^{89}$

ry Committee on the Framework Convention for the Protection of National Minorities, „Opinion on Germany“, ACFC/INF/OP/I (2002) 008; Im Fall des Lubicon Lake Band ging es um Enteignungen von $10.000 \mathrm{~km}^{2}$ zur Öl- und Gas-Förderung. UN Generalversammlung, „, Report of the Human Rights Committee", Communication No. 167/1984, Bernard Ominayak, Chief of the Lubicon Lake Band v. Canada (Lubicon Lake Band v. Canada), UN Doc.

A/45/40(Vol.II)(Supp), 4. Oktober 1990, Annex IX.A., S. 1.

${ }^{85}$ Nowak, U.N. covenant on civil and political rights, Art. 27, Rn. 47; Scherer-Leydecker, Minderheiten und sonstige ethnische Gruppen, S. $252 \mathrm{f}$.

86 Human Rights Committee, „General Comment No. 23: The rights of minorities (Art. 27)“, CCPR/C/21/Rev.1/Add.5, Abs. 6.1.

87 Tove H. Malloy, „The Title and the Preamble“, in: Marc D. Weller (Hrsg.), The rights of minorities: a commentary on the European Framework Convention for the Protection of National Minorities, 2006, S. 58.

${ }^{88}$ Nowak, U.N. covenant on civil and political rights, Art. 27, Rn. 48.

89 UN Generalversammlung, „Report of the Human Rights Committee“, Communication No. 197/1985, Kitok v. Sweden, UN Doc. A/43/40(Supp), 28. September 1988, Annex VII, S. 229, Abs. 9.4. 
A. Der internationale Minderheitenschutz

c) Aktive Schutz- und Unterstützungsmaßnahmen

Umstritten ist, inwieweit Minderheitenschutzpflichten auch zu positiven Unterstützungsmaßnahmen verpflichten. In seinem general comment zu Artikel 27 IPbpR führt das Human Rights Committee aus, dass

positive measures by States may also be necessary to protect the identity of a minority and the rights of its members to enjoy and develop their culture and language and to practise their religion, in community with the other members of the group. ${ }^{90}$

Auch in der Literatur wird die Auffassung vertreten, dass Sinn und Zweck des Minderheitenschutzes positive Maßnahmen von Seiten des Staates umfasst und somit die notwendigen Ressourcen bereitgestellt werden müssen. ${ }^{91}$ Mit dem Einwand der wirtschaftlichen Machbarkeit, ${ }^{92}$ dem negativ formulierten Wortlaut des Artikel 27 IPbpR sowie Verweisen auf die travaux préparatoires des IPbpR wird allerdings auch gegen eine solche progressive Auslegung des Minderheitenschutzes argumentiert. ${ }^{93}$ Im Verhandlungsprozess zu Artikel 27 IPbpR wurde ein von der Sowjetunion eingebrachter, auf positive staatliche Verpflichtungen gerichteter Vorschlag explizit mit vier zu acht Stimmen abgelehnt. ${ }^{94}$ Hintergrund waren Befürchtungen, durch positive Maßnahmen ein Minderheitenbewusstsein künstlich zu wecken und so Konflikte zu provozieren. ${ }^{95}$

Dennoch impliziert der Schutzzweck des Minderheitenschutzes nicht nur eine de jure Gleichstellung mit der Mehrheitsgesellschaft, sondern das Ziel einer de facto Gleichbehandlung. Insbesondere aus dem systematischen Verständnis im Verhältnis zum Diskriminierungsverbot ergibt sich ein weitergehender Schutzanspruch für den Minderheitenschutz. ${ }^{96}$ Rechtlich sind Minderheiten demnach gegebenenfalls besser zu stellen, um wirkliche Gleichberechtigung zu erreichen. Diese Auslegung wird explizit durch die nordischen Staaten unterstützt, die der Meinung

${ }^{90}$ Human Rights Committee, „, General Comment No. 23: The rights of minorities (Art. 27), CCPR/C/21/Rev.1/Add.5“, Abs. 6.2.

${ }^{91}$ Thornberry, ,The UN Declaration on the Rights of Persons Belonging to National or Ethnic, Religious and Linguistic Minorities: Background, Analysis, Observations, and an Update“, S. $24 \mathrm{f}$.

${ }^{92}$ Tomuschat, „Protection of Minorities under Article 27 of the International Covenant on Civil and Political Rights“, S. 968f.

${ }_{93}$ Scherer-Leydecker, Minderheiten und sonstige ethnische Gruppen, S. 254-261; M.w.N. Tomuschat, „Protection of Minorities under Article 27 of the International Covenant on Civil and Political Rights“, S. 969.

${ }^{94}$ Commission on Human Rights, „Ninth Session, Summary Record of the 371st Meeting“, UN Doc. E/CN.4/SR.371, 5. Oktober 1953, S. 6. Der Vorschlag lautete: „The State shall ensure to national minorities the right to use their native tongue and to possess their national schools, libraries, museums and other cultural and educational institutions."

${ }^{95}$ Nowak, U.N. covenant on civil and political rights, Art. 27, Rn. 39.

${ }^{96}$ Ebd., Art. 27, Rn. 40. 
sind, dass Artikel 27 IPbpR die Verantwortung für positive Unterstützungsmaßnahmen zum Erhalt und zur Entwicklung der ethnischen, kulturellen, sprachlichen und religiösen Identität impliziert. ${ }^{97}$

Bei den neueren Entwicklungen normativer Quellen des Minderheitenschutzes, insbesondere der UN Deklaration von 1992 und der Framework Convention, zeigt sich verstärkt die Bereitschaft zu aktiven und auch explizit finanziellen Unterstützungsmaßnahmen. So ermuntert beispielsweise Artikel 4 (3) der UN Minderheitendeklaration die Staaten explizit dazu, die Ausbildung der Sprache sicher zu stellen. ${ }^{98}$ Artikel 5 der UN Minderheitendeklaration schreibt fest, dass nationale Politiken und Programme unter Beachtung der Interessen von Minderheiten implementiert werden sollen. ${ }^{99}$ Auch Artikel 5 der Framework Convention schreibt die aktive Unterstützung von Minderheiten fest. Beispielsweise sollen die Gruppen in Entscheidungsprozesse finanzieller Unterstützungsmaßnahmen mit eingebunden werden oder diese selbst übernehmen. ${ }^{100}$

IV. Berichts- und Beschwerdeverfahren des internationalen Minderheitenschutzes

Der internationale Minderheitenschutz ist Teil des internationalen Systems der Menschenrechte und fügt sich in die Strukturen und Mechanismen der menschenrechtlichen Überwachungs- und Durchsetzungsinstitutionen ein. Das bedeutet, dass die Garantien des internationalen Minderheitenschutzes anders als reziproke völkerrechtliche Verträge in der Regel nicht im Rahmen der Regeln der Staatenverantwortlichkeit bilateral durch Retorsion oder Repressalie durchgesetzt werden. ${ }^{101}$

Die Aussetzung von staatlichen Menschenrechtsverpflichtungen in Reaktion auf etwaige Verletzungen menschenrechtlicher Verträge durch einen anderen Staat ist mit dem Schutzzweck der Menschenrechte nicht vereinbar. Die menschenrechtlichen Verpflichtungen stehen nicht in Reziprozität zueinander und geben somit nicht das Recht auf Aussetzung der eigenen Verpflichtungen bei Nichtein-

${ }^{97}$ Commission on Human Rights, „Fourty eighth Session, Summary Record of the 17th Meeting“, UN Doc. E/CN.4/1992/SR.17, 12. Februar 1992, Abs. 69.

98 UN Generalversammlung, ,Declaration on the Rights of Persons Belonging to National or Ethnic, Religious and Linguistic Minorities“, UN Doc. A/Res/47/135, Art. 4 Abs. 3; Thornberry, „The UN Declaration on the Rights of Persons Belonging to National or Ethnic, Religious and Linguistic Minorities: Background, Analysis, Observations, and an Update", S. 21 ff, 25; Nowak, U.N. covenant on civil and political rights Art. 27, Rn. 52.

${ }^{99}$ UN Generalversammlung, „Declaration on the Rights of Persons Belonging to National or Ethnic, Religious and Linguistic Minorities“, UN Doc. A/Res/47/135, Art. 5; Nowak, U.N. covenant on civil and political rights, Art. 27, Rn. 52.

100 Advisory Committee on the Framework Convention for the Protection of National Minorities, „Opinion on Finland“, ACFC/INF/OP/I (2001) 002, 22. September 2000, Abs. 23; Gilbert, „Article 5“, S. 160-162.

101 Dinah Shelton, Remedies in international buman rights law, 2005, S. 97-102. 
A. Der internationale Minderheitenschutz

haltung der Verträge durch die anderen Vertragsparteien, da so die eigentliche Funktion menschenrechtlicher Garantien untergraben werden würde. Menschenrechte sind repressalienfest. Da die klassischen Instrumente der völkerrechtlichen Durchsetzung somit keine Anwendung finden, wurden im Bereich des Menschenrechtsschutzes neue Mechanismen entwickelt, die durch Überwachungs- und Berichtsverfahren Verletzungen feststellen und durch die Veröffentlichung politischen Druck zur Einhaltung erzeugen. ${ }^{102}$

Unter den klassischen Mechanismen und auch im Rahmen der internationalen Berichts- und Beschwerdeverfahren stellt sich ein weiteres Problem. Wenn ein Staat die Menschen- und Minderheitenrechte eigener Staatsangehöriger verletzt und keine Verbindung zu Drittstaaten besteht, mangelt es im System der Staatenverantwortlichkeit an der Beschwerdebefugnis Dritter Staaten. Diese können sich mangels einer rechtlichen Verbindung nicht für die Belange verletzter Individuen oder Gruppen einsetzen. Allerdings, so konkretisierte es die International Law Commission in ihren Articles on State Responsibility, wirken Menschenrechtsverträge, und somit auch die Garantien des Minderheitenschutzes, in ihrer Funktion erga omnes, so dass Menschenrechtsverletzungen eines Staates gegenüber den eigenen Staatsangehörigen auch alle anderen Mitgliedstaaten eines entsprechenden multilateralen Menschenrechtsvertrags verletzt und somit eine Beschwerdebefugnis für letztere gegeben ist. ${ }^{103}$

Bei den unterschiedlichen Minderheitenschutzinstrumenten stehen verschiedene Möglichkeiten der Berichts- und Beschwerdeverfahren zur Verfügung, die allein durch die Öffentlichmachung von Verletzungen wirken. Näher dargestellt werden im Folgenden die Institutionen der UN Gremien, das Beschwerdeverfahren unter dem ersten Zusatzprotokoll des IPbpR, das Berichtsverfahren unter der Framework Convention, die Arbeit des European High Commissioner sowie die Durchsetzungsmechanismen der bilateralen Verträge in Europa.

\section{UN Institutionen}

Unter dem Dach der Vereinten Nationen entwickelte sich eine Reihe von Institutionen, die Beschwerden annehmen und Berichte zu Verletzungen von Minderheitenrechten zusammenstellen. ${ }^{104}$ Die über mehrere Jahrzehnte gewachsene Architektur des UN Menschenrechtssystems wurde zu Beginn des neuen Jahrtausends grundlegend reformiert und neu strukturiert. Heute befassen sich vorrangig das Forum on Minority Issues und der Independent Expert on Minority Issues

\footnotetext{
102 Ebd., S. 99.

103 James Crawford, The International Law Commission's articles on state responsibility, 2002, S. 277; Shelton, Remedies in international buman rights law, S. 98.

${ }^{104}$ Gudmundur Alfredsson, „Minority Rights at the United Nations“, in: Daniel Thürer und Zdzisław Kędzia (Hrsg.), Managing diversity : protection of minorities in international law, 2009, S. 24-28.
} 
unter der Leitung des Human Rights Council mit den Fragen des Minderheitenschutzes. Normative Grundlage der Arbeit im Bereich des Minderheitenschutzes ist dabei vorrangig die UN Minderheitendeklaration von 1992. ${ }^{105}$ Personell und institutionell unterstützt werden diese Institutionen durch das Office of the High Commissioner for Human Rights. ${ }^{106}$

a) Historische Struktur der UN Institutionen des Minderheitenschutzes

Das zentrale Gremium der Arbeiten der UN in Menschenrechtsfragen war bis 2006 die Human Rights Commission, die durch den neuen Human Rights Council ersetzt wurde. ${ }^{107}$ Ihr untergeordnet diente die Sub-Commission on the Promotion and Protection of Human Rights (vormals Sub-Commission on Prevention of Discrimination and Protection of Minorities) als, think tank' und Träger der Working Group on Minorities (1995-2007). Die Sub-Commission wird heute vom Human Rights Council Advisory Committee ersetzt, welches die Aufgaben der Beratung zu Fragen des Menschenrechtsschutzes wahrnimmt. ${ }^{108}$

Im Bereich des Minderheitenschutzes etablierte der Human Rights Council damals noch als Human Rights Commission - bereits im Juli 2005 das Amt des UN Independent Expert on Minority Issues, der vom High Commissioner for Human Rights ernannt wird. ${ }^{109}$ Des Weiteren gründete der Human Rights Council als Ersatz der Working Group on Minorities das Forum on Minority Issues, welches im September 2007 seine Arbeit aufgenommen hat. ${ }^{110}$ Heute wirken somit im Bereich des Minderheitenschutzes als Unterorgane des Human Rights Council der Independent Expert on Minority Issues und das Forum on Minority Issues.

b) Forum on Minority Issues

Das Forum on Minority Issues trifft sich jährlich für zwei Tage in Genf, um unter aktiver Beteiligung von Staatenvertretern, Vertretern von UN Institutionen, internationalen und regionalen Organisationen, nationalen Menschenrechtsorganisation, Vertretern von Minderheiten, Experten aus der Wissenschaft und NGOs die Situation von Minderheiten unter einem Themenschwerpunkt zu diskutieren. Ein Bericht und die während der Sitzungen erarbeiteten Vorschläge

\footnotetext{
105 UN Generalversammlung, „,Declaration on the Rights of Persons Belonging to National or Ethnic, Religious and Linguistic Minorities“, UN Doc. A/Res/47/135.

106 UN Generalversammlung, „High Commissioner for the Promotion and Protection of all Human Rights“, UN Doc. A/Res/48/141, 20. Dezember 1993.

107 UN Generalversammlung, „Human Rights Council“, UN Doc. A/Res/60/251, 3. April 2006.

${ }^{108}$ Human Rights Council, „Institution-Building of the United Nations Human Rights Council“, UN Doc. A/HRC/RES/5/1, 18. Juni 2007.

${ }^{109}$ Commission on Human Rights, „Report on the Sixty-First Session“, UN Doc.

E/CN.4/2005/135, 16. Dezember 2005, S. 9 und Abs. 447, S. 439.

${ }^{110}$ Human Rights Council, „Forum on Minority Issues“, UN Doc. A/HRC/RES/6/15, 28. September 2007.
} 
A. Der internationale Minderheitenschutz

werden veröffentlicht und als Teil des Berichts des Independent Expert dem Human Rights Council zugeleitet.

c) Independent Expert on Minority Issues

Der Independent Expert on Minority Issues bündelt Informationen von Staaten, Experten, UN Organen, internationalen und regionalen Organisationen, NGOs und weiteren zivilgesellschaftlichen Organisationen und richtet auf dieser Grundlage entsprechende communications zur Umsetzung der UN Minderheitendeklaration an die Staaten. Er verfasst und veröffentlicht einen jährlichen Bericht zu den Bereichen seines Mandats sowie konkrete thematische Studien, die dem Human Rights Council zugeleitet werden. Auf Einladung von Staaten besucht er darüber hinaus Länder für Anhörungen, um unterschiedliche Programme voranzutreiben und Beschwerden aufzunehmen.

d) Human Rights Council

Im Human Rights Council werden die Ergebnisse dieser Verfahren durch entsprechende Berichte und Diskussionen zusammengeführt. Er besteht aus 47 Staatenvertretern, die unter Berücksichtigung geographischer Verteilung für maximal zwei Amtsperioden von je drei Jahren mit einfacher Mehrheit von der UN Generalversammlung gewählt werden. ${ }^{111}$ Des Weiteren betreut der Human Rights Council den im März 2006 eingeführten Universal Periodic Review, ein Staatenberichtsverfahren zur Menschenrechtssituation aller UN Mitgliedstaaten, welches aller vier Jahre von jedem Staat erstellt wird. ${ }^{112}$ Der Großteil der inhaltlichen Arbeit zum Minderheitenschutz in der UN findet allerdings bei den beiden speziellen Institutionen, dem Forum on Minority Issues und dem Independent Expert on Minority Issues, statt.

e) Complaint Procedure im Human Rights Council

Im Human Rights Council besteht außerdem die Möglichkeit der Individualbeschwerde, die im Rahmen der complaint procedure vorgebracht werden kann. ${ }^{113}$ Dieses Verfahren wurde 2007 eingeführt und ersetzt das frühere sogenannte 1503 Verfahren. Die Beschwerden werden zunächst von der Working Group on Communications (WGC), einem Gremium aus fünf für je drei Jahre (einmalig verlängerbar) gewählten Mitgliedern des Human Rights Council Advisory Committee, auf ihre Zulässigkeit überprüft. ${ }^{114}$ Zulässig sind Beschwerden bei stetigen, schweren

\footnotetext{
111 UN Generalversammlung, „Human Rights Council“, UN Doc. A/Res/60/251, Abs. 7.

112 Ebd., Abs. 5 (e).

113 Meghna Abraham, A new chapter for buman rights: a bandbook on issues of transition from the Commission on Human Rights to the Human Rights Council, 2006, S. 62-71.

114 Human Rights Council, „Institution-Building of the United Nations Human Rights Council“, A/HRC/RES/5/1, Abs. 91-95.
} 
und glaubwürdig belegten Menschenrechtsverletzungen. ${ }^{115}$ Auf Grundlage der Informationen und Empfehlungen der $W G C$, welche sich zweimal pro Jahr für je fünf Tage trifft, werden die Beschwerden von der Working Group on Situations (WGS) unter Berücksichtigung von entsprechenden Kommentaren der betroffenen Staaten zu einem Bericht an den Human Rights Council zusammengefasst und mit Empfehlungen zum weiteren Vorgehen kommentiert. Das WGS besteht aus fünf Mitgliedern, die jeweils von einer der regionalen UN Gruppen im Human Rights Council für eine Amtszeit von einem Jahr (einmalig verlängerbar) bestimmt werden. Sie treffen sich ebenso wie das WGC zwei Mal pro Jahr für je fünf Tage. ${ }^{116}$ Im Human Rights Council wird der Bericht der WGS nichtöffentlich diskutiert und darauf basierend eine Entscheidung getroffen. ${ }^{117}$

\section{Berichts- und Beschwerdemechanismen unter dem IPbpR}

Das Kontrollorgan des Internationalen Pakts über bürgerliche und politische Rechte, dem mit seinem Artikel 27 wichtigsten normativen Instrument des internationalen Minderheitenschutzes, ist das Human Rights Committee. ${ }^{118}$ Es begutachtet die regelmäßig einzureichenden Staatenberichte und entscheidet über zwischenstaatliche Beschwerden sowie Beschwerden von Individuen unter dem ersten Zusatzprotokoll. ${ }^{119}$ Das Human Rights Committee besteht aus 18 unabhängigen Experten, die auf je vier Jahre von den Mitgliedstaaten des IPbpR gewählt werden. ${ }^{120}$

Mitgliedstaaten müssen gemäß Artikel $40 \mathrm{IPbpR}$ innerhalb eines Jahres nach Beitritt und daraufhin regelmäßig - in der Regel alle vier Jahre - Berichte zur Menschenrechtssituation in ihrem Land einreichen. Diese werden vom Human Rights Committee begutachtet und in Hinblick auf etwaige Bedenken mit Empfehlungen in Form der concluding observations beantwortet. Auf Grundlage dieser Berichte verfasst das Human Rights Committee auch seine general comments, die als juristische Kommentare den Schutzumfang der einzelnen Menschenrechtsgarantien des IPbpR weiter ausdifferenzieren. Für den Minderheitenschutz ist dies insbesondere der general comment No. 23: The rights of minorities. ${ }^{121}$

Artikel 41-43 IPbpR regeln die Möglichkeit der Staatenbeschwerden gegenüber einem anderen Mitgliedstaat an das Human Rights Committee. Das Instrument der zwischenstaatlichen Beschwerde wurde bisher jedoch noch nie angewandt, da

\footnotetext{
115 Ebd., Abs. 85.

116 Ebd., Abs. 96-99.

117 Ebd., Abs. 104.

118 IPbpR, Art. 28-39.

119 Optional Protocol to the International Covenant on Civil and Political Rights (Fakultativproto-

koll zum Internationalen Pakt über bürgerliche und politische Rechte), 999 UNTS 302,

16. Dezember 1966.

120 IPbpR, Art. 28.

121 Human Rights Committee, „General Comment No. 23: The rights of minorities (Art. 27)“, CCPR/C/21/Rev.1/Add.5.
} 
A. Der internationale Minderheitenschutz

explizite Beschwerden über diesen Mechanismus die existierenden politischen und diplomatischen Beziehungen zu sehr belasten würden. ${ }^{122}$

Unter dem ersten Zusatzprotokoll des IPbpR besteht die Möglichkeit der Beschwerde von betroffenen Individuen an das Human Rights Committee. Mehr als zwei Drittel der Mitgliedstaaten des IPbpR sind dem Zusatzprotokoll und damit einem der wichtigsten Individualbeschwerdemechanismen des internationalen Menschenrechtsschutzes beigetreten. ${ }^{123}$ Das Verfahren findet ausschließlich schriftlich und vertraulich statt. Formlos eingereichte Beschwerden werden vom Sekretariat dem zuständigen Committee Mitglied zugeleitet und zunächst auf ihre Zulässigkeit hin überprüft, bevor sie inhaltlich behandelt werden. In der Praxis werden diese beiden Schritte allerdings gemeinsam entschieden. ${ }^{124} \mathrm{Um}$ die weitgehende Unabhängigkeit des Committees zu gewährleisten, nimmt der Vertreter eines ,angeklagten' Staates nicht am Verfahren gegen seinen Heimatstaat teil. ${ }^{125}$ Die Entscheidungen, seit 1982 auch solche zu unzulässigen Beschwerden, werden schließlich veröffentlicht. Obwohl das Zusatzprotokoll dabei nur von views spricht, nehmen die veröffentlichten Entscheidungen die Form von quasigerichtlichen Urteilen an und enthalten Ausführungen zu den Tatsachen und rechtlichen Erwägungen. Den Mitgliedern des Human Rights Committees bleibt dabei vorbehalten abweichende Meinungen zu publizieren. ${ }^{126}$

\section{Berichtsverfahren der Framework Convention}

Der Überwachungsmechanismus der Framework Convention ist eine Einrichtung des Europarates und wird vom Ministerkomitee ausgeführt. ${ }^{127}$ Dieses Gremium der Außenminister der Mitgliedstaaten wird dabei von einem Beratungsausschuss unterstützt. ${ }^{128}$ Jeder neue Mitgliedstaat muss ein Jahr nach Beitritt sowie daraufhin in der Regel alle fünf Jahre oder auf Anfrage einen Bericht einreichen. Der Bericht wird den 18 Mitgliedern des Beratungsausschusses zugeleitet, die in einer länderspezifischen Arbeitsgruppe auch die umfangreichen Anhänge der Berichte von NGOs und Organisationen von Minderheiten bearbeiten. ${ }^{129}$ Die Arbeitsgruppe bereitet auf Grundlage dieser Daten weitere Rückfragen an den jeweiligen Staat vor und führt Besuche in den betroffenen Ländern durch, bei

\footnotetext{
122 Nowak, U.N. covenant on civil and political rights, Art. 41, Rn. 2.

123 Ebd., Preamble First OP, Rn. 1-2.

124 Ebd., Preamble First OP, Rn. 2.

125 Human Rights Committee, „Rules of Procedure of the Human Rights Committee“, CCPR/C/3/Rev.3, 24. Mai 1994, Art. 84 (1)(a).

126 Nowak, U.N. covenant on civil and political rights, Preamble First OP, Rn. 2.

127 Die Regelungen dazu finden sich in Teil IV, Art. 24-26 FCNM.

128 Rainer Hofmann, ,"The Framework Convention for the Protection of National Minorities“, in: Bernd Rechel (Hrsg.), Minority Rights in Central and Eastern Europe, 2009, S. 46; Letschert, The impact of minority rights mechanisms, S. 145-221.

${ }^{129}$ Letschert, The impact of minority rights mechanisms, S. 154, 164.
} 
denen Behörden sowie Repräsentanten von Minderheiten und der Zivilgesellschaft gehört werden. Der Entwurf der Arbeitsgruppe wird im Plenum des Beratungsausschusses diskutiert und - sofern angenommen - der Rapporteur Group on Human Rights, einem Unterkomitee des Ministerkomitees, weitergeleitet. Schließlich nimmt das Ministerkomitee den Bericht mit einer spezifischen Resolution an, welche Ergebnisse und Hinweise formuliert. Jeweilige Folgeberichte nehmen Bezug auf die vorausgegangenen Berichte dieses Überwachungsmechanismus'. ${ }^{130}$

\section{Der European High Commissioner on National Minorities der OSZE}

Mit dem Amt des High Commissioner on National Minorities steht im Rahmen der OSZE eine Institution zur Verfügung, die sich hauptsächlich mit Konfliktprävention bei Streitigkeiten um Minderheiten befasst. ${ }^{131}$ Die Aufgabe des High Commissioner besteht dabei zum einen darin, sich ethnischer Konflikte anzunehmen und bei Spannungen deeskalierend einzugreifen. Zum anderen soll er die OSZE vor ausbrechenden Konflikten warnen. ${ }^{132} \mathrm{Er}$ arbeitet unabhängig von staatlichen Einflüssen und führt direkte diplomatische Verhandlungen zwischen beteiligten Parteien und kann auch im Rahmen der OSZE Informationen öffentlich machen. ${ }^{133}$

\section{Durchsetzung bilateraler Verträge}

Obwohl einige der bilateralen Verträge in Europa die Verbindlichkeit ihres Inhalts durch Formulierungen gegenseitiger Verpflichtungen bekräftigen, ergeben sich auch hier durch den menschenrechtlichen Charakter des Minderheitenschutzes andere Wege der Durchsetzung als die reziproke Aussetzung entsprechender Verpflichtungen. ${ }^{134}$ Für zwischenstaatliche Konflikte haben sich dabei bilaterale Mechanismen zur Streitbeilegung gegenüber institutionellen Streitschlichtungsmechanismen durchgesetzt. Diese Verfahren können durch Dialog und gegenseitiges Einverständnis zu Lösungen kommen, welche die beteiligten Staaten direkter beeinflussen können. Insbesondere sind dies Verfahren von regelmäßigen diplomatischen Konsultationen, nationale Überwachungs- und Umsetzungsmechanismen und bilaterale Kommissionen. ${ }^{135}$ Einige wenige der bilateralen Kommissionen sehen dabei auch die Beteiligung von nicht-

\footnotetext{
${ }^{130}$ Hofmann, „The Framework Convention for the Protection of National Minorities“, S. 47.

131 Conference for Security and Co-operation in Europe, „Helsinki Document 1992, The Challenges of Change“, 9. Juli 1992, Chapter II, Abs. 2-3; Arie Bloed, „The OSCE and the Issue of National Minorities“, in: Alan Phillips und Allan Rosas (Hrsg.), Universal Minority Rights, 1995, S. 113.

${ }^{132}$ Krzysztof Drzewicki, „Minority Protection within the OSCE“, in: Daniel Thürer und Zdzisław Kędzia (Hrsg.), Managing diversity: protection of minorities in international law, 2009, S. $121 \mathrm{f}$. 133 Ebd., S. $122 \mathrm{f}$.

134 Arp, International norms and standards for the protection of national minorities, S. 31-34.

135 Ebd., S. 34-44.
} 
A. Der internationale Minderheitenschutz

staatlichen Akteuren vor und nehmen Beschwerden von Betroffenen entgegen. ${ }^{136} \mathrm{Im}$ Vergleich mit den etablierten Verfahren und Organen der multilateralen Instrumente bieten die Ansätze der Streitbeilegung bilateraler Verträge für die betroffenen Gruppen weniger institutionalisierte Möglichkeiten etwaige Verletzungen von Minderheitenrechten international geltend zu machen.

\section{Ergebnis}

Die Garantien des internationalen Minderheitenschutzes können weder bei bilateralen Verträgen von einer der Vertragsparteien noch im Rahmen multilateraler Verträge durch die völkerrechtlichen Instrumente der Repressalie oder Retorsion, wie bei reziproken internationalen Verpflichtungen, durchgesetzt werden. Die Kontrolle des Minderheitenschutzes erfolgt im Rahmen des internationalen Systems der Menschenrechte vielmehr durch teilweise quasi-gerichtlich ausgestaltete Berichts- und Beschwerdemechanismen. ${ }^{137}$ Problematisch ist dabei die immer noch nicht umfassend globale Ratifikation menschenrechtlicher Verträge und Zusatzprotokolle für Beschwerdemechanismen, wobei insbesondere in Südostasien die Ratifizierungszahlen von Menschenrechtsverträgen sehr niedrig sind. ${ }^{138}$ Bei der Veröffentlichung von Verletzungen, bei politischen Kampagnen und auch bei der Ausübung und Wahrnehmung von Rechten vor internationalen Institutionen spielen dabei die zahlreichen Nichtregierungsorganisationen eine entscheidende Rolle. ${ }^{139}$

\section{Zusammenfassung: ältestes Konzept zum Schutz kulturell distinkter Gruppen}

Der internationale Minderheitenschutz bildet das älteste völkerrechtliche Konzept kulturell distinkter Gruppen auf substaatlicher Ebene. Nach seinen Anfängen im Bereich der kollektiven Religionsfreiheit entwickelte der Minderheitenschutz einen umfänglichen Schutzbereich für Sprache, Religion und die kulturelle Integrität, der im modernen Völkerrecht grundlegende Garantien für das innerstaatliche Miteinander kulturell distinkter Gruppen gibt. Insbesondere die Entwicklungen der letzten 20 Jahre in Süd-Osteuropa und Zentralasien haben die Notwendigkeit rechtlicher Garantien für Minderheiten abermals deutlich gemacht. Durch den Minderheitenschutz sollen solche Spannungen zwischen Mehr- und Minderheiten ausgeglichen werden, die sich aus der völkerrechtlichen Struktur der staatlichen Machtkonzentration ergeben. ${ }^{140}$

\footnotetext{
136 Ebd., S. 44f.

137 Letschert, The impact of minority rights mechanisms, S. 4.

138 Mit einer entsprechenden Aufstellung: Joshua Castellino, Minority rights in the Pacific region: a comparative analysis, 2009, S. $15 \mathrm{f}$.

139 Alfredsson, „Minority Rights at the United Nations“, S. 28.

140 Manfred Mohr, Friedenssichernde Aspekte des Minderbeitenschutz̨es in der Ära des Völkerbundes und der Vereinten Nationen in Europa, 1996.
} 


\section{B. Die Rechte indigener Völker}

Indigene Völker spielen seit der Entdeckung der ,Neuen Welt' eine Rolle im Völkerrecht. Nachdem sie die meiste Zeit Objekt völkerrechtlicher Regelungen waren, etablierten sich Vertreter indigener Gruppen seit den 1970er Jahren als fester Bestandteil im institutionellen Gefüge internationaler Organisationen. Indigene treten heute auch selbst, als agierende Subjekte, für ihre Belange ein. ${ }^{141}$ Dieser Politikwechsel von den historischen Verbrechen der Assimilationspolitik und der Unterdrückung hin zur Wertschätzung indigener Kultur und zur aktiven Beteiligung indigener Völker spiegelt sich deutlich in den völkerrechtlichen Primärquellen wider. So verankerte die globale Indigenenbewegung insbesondere den Anspruch auf das Selbstbestimmungsrecht indigener Völker im völkerrechtlichen Diskurs. Im Folgenden sollen der Geschichte, dem Bedeutungsgehalt des Selbstbestimmungsrechts indigener Völker sowie den bis heute entwickelten Um- und Durchsetzungsmechanismen ihrer Rechte nachgegangen werden.

\section{Die Geschichte indigener Völker im Völkerrecht}

Die Geschichte indigener Völker im Völkerrecht begann mit den Theorien zur Rechtfertigung der Eroberung und Besiedlung Amerikas. Die führenden Völkerrechtstheoretiker des Spätmittelalters und der frühen Neuzeit, Papst Innozenz IV, Francisco de Vitoria, Hugo Grotius sowie Emer de Vattel, erarbeiteten argumentative Strukturen, um die Expansionspolitiken der Europäer normativ zu rechtfertigen, Regelungen für einen ,gerechten', aber eigentlich praktikablen Umgang mit den Menschen vor Ort vorzugeben und damit letztlich die Vorherrschaft ihrer Heimatstaaten gegenüber den anderen europäischen Siedlern zu erlangen und zu behaupten. ${ }^{142}$ Die ersten Theorien von Papst Innozenz IV beruhten auf der Überzeugung, die Kirche habe de jure und a priori die Rechtsprechung und Gewalt über die ganze Welt inne und könne diese Herrschaftsansprüche de facto durchsetzen, sollten sich die ,Ungläubigen' weigern, die Predigt des Evangeliums zuzulassen. ${ }^{143}$ Trotz der formellen Anerkennung des dominium der Nicht-Christen genügten fadenscheinige Gründe, um diese in einem ,gerechten Krieg' bekämpfen zu dürfen. ${ }^{144}$ Ausgehend vom universellen a priori Herrschaftsanspruch der Kirche sprach der Papst den spanischen und portugiesi-

\footnotetext{
${ }^{141}$ Russel Lawrence Barsh, „Indigenous Peoples in the 1990s: From Object to Subject in International Law?", Harvard Human Rights Journal, Vol. 7 (1994), S. 33-86.

142 Robert J. Miller u. a., Discovering Indigenous Lands: The Doctrine of Discovery in the English Colonies, 2010, S. 1-25; Mark D. Cole, Das Selbstbestimmungsrecht indigener Völker, 2009, S. 231-238.

${ }^{143}$ Innozenz IV, Commentaria super libros quinque decretalium, 1570; Jörg Fisch, Die europäische Expansion und das Völkerrecht, 1984, S. 187-193.

144 Jörg Fisch, Die europäische Expansion und das Völkerrecht, S. $188 \mathrm{f}$.
} 
B. Die Rechte indigener Völker

schen Königen in Form von päpstlichen Bullen das Recht zu, die Ungläubigen bekämpfen und ihnen ihr dominium entziehen zu dürfen, sollten sie sich dem christlichen Glauben widersetzen. ${ }^{145}$ Diese ungehinderte Expansionspolitik hatte ein gewaltsames und brutales Vorgehen in Amerika zur Folge, welches von einer Oppositionsbewegung in Spanien angegriffen wurde und eine eingehende Begründung der Rechtfertigung erforderlich machte. ${ }^{146}$

Eine Schlüsselrolle in den Auseinandersetzungen zur theoretischen Begründung der Eroberung der, neuen Welt' nahm im 16. Jahrhundert der Theologe und Jurist Francisco de Vitoria ein. In seiner Vorlesung ,Über die kürzlich entdeckten Indianer von 1539 stellte er eine Vielzahl von Argumenten für und wider der Expansionspolitik dar. ${ }^{147}$ In der Tradition von Innozenz IV erkennt Vitoria darin zwar grundsätzlich die Souveränitäts- und Besitzrechte der Indianer an und erklärt, dass die Indianer weder zum Glauben verpflichtet sind noch dazu gezwungen werden könnten. ${ }^{148}$ Er erklärt aber ebenso, dass eine Behinderung der aus dem Naturrecht erwachsenden Reise- und Niederlassungsfreiheit oder eine Behinderung der Verkündigung des Evangeliums den Spaniern das Recht zu einem ,gerechten Krieg' gegen die Indianer gebe. ${ }^{149}$ Er begründete die spanische Herrschaft mit einer naturrechtlichen Rechtfertigung und entzog somit der Kirche das Recht, die Eroberung neuen Landes jeweils im Einzelfall über päpstliche Bullen bewilligen zu können. ${ }^{150}$

Der Grundgedanke einer weiteren wichtigen Argumentationslinie zur Rechtfertigung der Landnahme findet sich ebenfalls bereits bei Vitoria. Er argumentierte, dass die Erstentdeckung und Inbesitznahme herrenloser Gebiete einen Anspruch auf das Land begründen könne. Diese Konstellation liege jedoch im Falle Amerikas mit seiner vorherigen Besiedelung durch die Ureinwohner nicht vor. ${ }^{151}$ Diese Rechtsansicht für herrenlose beziehungsweise unbewohnte Gebiete findet sich auch bei Hugo Grotius. Seine naturrechtliche Argumentation zur Frage der Besetzung von unbewohntem Land und zur Rechtfertigung eines, gerechten Krie-

\footnotetext{
145 Ebd., S. 205-209.

146 Cole, Das Selbstbestimmungsrecht indigener Völker, S. 232.

147 Francisco de Vitoria, De Indis recenter inventis et de jure belli hispanorum in barbaros, Walter Schätzel (Hrsg.) 1539/1952.

148 Francisco de Vitoria, De Indis recenter inventis et de jure belli hispanorum in barbaros, Walter Schätzel (Hrsg.), S. 45, 82f.; Miller u. a., Discovering Indigenous Lands, S. 14; Fisch, Die europäische Expansion und das Völkerrecht, S. 212-215.

${ }^{149}$ Francisco de Vitoria, De Indis recenter inventis et de jure belli hispanorum in barbaros, Walter Schätzel (Hrsg.), S. 99f., 107; Paul Keal, ,,Just Backward Children“: International Law and the Conquest of Non-European Peoples“, in: Anthony J. Connolly (Hrsg.), Indigenous Rights, 2009, S. 52.

150 Miller u. a., Discovering Indigenous Lands, S. 14; S. James Anaya, Indigenous peoples in international law, 2004, S. 17.

151 Francisco de Vitoria, De Indis recenter inventis et de jure belli hispanorum in barbaros, Walter Schätzel (Hrsg.), S. 45; Fisch, Die europäische Expansion und das Völkerrecht, S. 214.
} 
ges' war zwar insgesamt uneinheitlich. ${ }^{152}$ Allerdings machte er deutlich, dass für die Begründung der Besitznahme von Gütern und Land die Unterscheidung zwischen ,gerechtem' und ,ungerechtem' Krieg im jus gentium, also im Völkerrecht, keine Bedeutung habe und jedweder Besitz- und Landerwerb, der im Rahmen eines ,förmlichen Krieges“ erobert werde, Bestand habe. ${ }^{153}$ „Dieser von Grotius eingeführte Dualismus von Naturrecht und Völkerrecht, von gerechtem und förmlichem Krieg“ bestimmte die Lehre des 17. und 18. Jahrhunderts. ${ }^{154}$ Zwar wirkten auch die Argumente zum ,gerechten Krieg' fort, die Argumentation Grotius' zum Besitzerwerb wurde aber ebenso verfolgt und beispielsweise von Emer de Vattel 1758 folgendermaßen verdeutlicht: ,Jede Erwerbung in einem förmlichen Kriege ist also gemäß dem freiwilligen Völkerrecht rechtsgültig unabhängig von der Gerechtigkeit der Sache und den Gründen." ${ }^{155}$

De Vattel vertrat auch eine konkretisierende Argumentation zur Doktrin terra nullius, dem Recht der Besiedelung von herrenlosen Gebieten. Danach sei nur solches Land terra nullius, welches keine wirkliche Besiedelung im Sinne einer Kultivierung des Landes und eines Gemeinwesens aufweise, welches dem in Europa vorherrschenden Ansatz der Besiedelung und der Landwirtschaft zumindest ähnelte. ${ }^{156}$ Diese Argumentation unterstützte die Position Englands und Frankreichs in ihren Kolonisationsvorhaben, da Nordamerika aufgrund des verschwenderischen Umgangs mit dem Land der indigenen Bevölkerung somit als terra nullius eingestuft und von den Europäern in Besitz genommen und besiedelt werden konnte. ${ }^{157}$

Die dargestellten Begründungsmuster zur Besiedelung und Eroberung der Kolonien durch die Europäer dienten nicht nur der moralisch-rechtlichen Rechtfertigung des gewaltsamen Vorgehens, sondern auch einer effizienten Aufteilung der Gebiete unter den Europäern. Dies zeigt sich auch in den verschiedenen Ritualen, wie dem Setzen von Flaggen oder Beschriften von Steintafeln, die eine Erstentdeckung den europäischen Kontrahenten gegenüber deutlich machen sollten. ${ }^{158}$

152 Hugo Grotius, De Jure Belli Ac Pacis, Walter Schätzel (Hrsg.), 1625/1950; Keal, ,,Just Backward Children': International Law and the Conquest of Non-European Peoples“, S. 54; Fisch, Die europäische Expansion und das Völkerrecht, S. 247.

153 Hugo Grotius, De Jure Belli Ac Pacis, Walter Schätzel (Hrsg.), S. 463; Cole, Das Selbstbestimmungsrecht indigener Völker, S. 236; Fisch, Die europäische Expansion und das Völkerrecht, S. 170.

${ }^{154}$ Fisch, Die europäische Expansion und das Völkerrecht, S. 171.

155 Emer de Vattel, Le droit des gens ou Principes de la loi naturelle appliqués à la conduite et aux affaires des nations et des souverains, Walter Schätzel (Hrsg.), 1758/1959, S. 472.

156 Emer de Vattel, Le droit des gens ou Principes de la loi naturelle appliqués à la conduite et aux affaires des nations et des souverains, Walter Schätzel (Hrsg.), S. 141f.; Cole, Das Selbstbestimmungsrecht indigener Völker, S. 237; Keal, ,,Just Backward Children': International Law and the Conquest of NonEuropean Peoples", S. 54; Anaya, Indigenous peoples in international law, S. 23.

157 Miller u. a., Discovering Indigenous Lands, S. 17-21; Cole, Das Selbstbestimmungsrecht indigener Völker, S. 238

158 Miller u. a., Discovering Indigenous Lands, S. 21f. 
Durch die gewaltsame Unterdrückung und Vernichtung indigener Völker und durch die nationalen Politiken einer Assimilation dieser Gruppen zu Arbeits- und Lebensgewohnheiten der weißen Mehrheit verschwanden sie bis zur Zeit des Völkerbundes aus den völkerrechtlichen Diskussionen. ${ }^{159}$

Mit dem Völkerbund entwickelte sich im Zuge der Neuordnung Europas nach dem Ersten Weltkrieg das Konzept des Selbstbestimmungsrechts der Völker. Vor diesem Hintergrund wurde im Prozess der Dekolonisierung der folgenden Jahrzehnte auch die Assimilationspolitik indigener Völker zunehmend hinterfragt. ${ }^{160}$ In der Satzung des Völkerbunds wurde den colonies, native inbabitants, indigenous populations und peoples not yet able to stand by themselves eine gerechte Behandlung durch die sie verwaltenden Staaten versprochen. ${ }^{161}$ Für die Mandatsgebiete, die ihre Unabhängigkeit noch nicht bis 1919 erstritten hatten, wurde - wenn auch nur auf dem Papier - eine Perspektive der Unabhängigkeit und Selbstbestimmung der Völker in den gefestigten, durch die Kolonialstaaten festgelegten Grenzen verankert. ${ }^{162}$ Voraussetzung für eine Unabhängigkeit war jedoch die ,Entwicklung und Zivilisierung' der Indigenen.

Pursuant to the civilizing mission, government and Christian church agents proceeded through the early part of the twentieth century to break down indigenous forms of political and social organization, disrupt communal land-holdings, and suppress cultural practices. ${ }^{163}$

Die politische Kehrtwende im Umgang mit Indigenen vollzog sich erst durch die Entwicklungen der normativen Werteordnung des Völkerrechts mit den internationalen Menschenrechten und der zunehmenden Präsenz indigener Völker auf der internationalen Bühne im institutionellen Gefüge der Vereinten Nationen. ${ }^{164}$ Bis in die zweite Hälfte des 20. Jahrhunderts wurde noch die Assimilationspolitik verfolgt, welche die Indigenen, zivilisieren und entwickeln' wollte. ${ }^{165}$ Das Canadian Indian residential school system, das obligatorische Internatssystem für indigene Kinder, und ähnliche Programme in den USA, Australien und Neusee-

\footnotetext{
159 Anaya, Indigenous peoples in international law, S. 26-33.

${ }^{160}$ Russel Lawrence Barsh, „Indigenous Peoples“, in: Daniel Bodansky, Jutta Brunnée, und Ellen Hey (Hrsg.), The Oxford handbook of international environmental law, 2007, S. 830; Cole, Das Selbstbestimmungsrecht indigener Völker, S. 249.

${ }^{161}$ Friedensvertrag von Versailles, Art. 1 bis 26: Satzung des Völkerbundes, 225 CTS 188, 28. Juni 1919, Art. 22 und 23; Barsh, „Indigenous Peoples“, S. 831.

162 James Summers, Peoples and international law, 2007, S. 134-39; Jérémie Gilbert, Indigenous peoples' land rights under international law, 2006, S. 14, 34f.

163 Anaya, Indigenous peoples in international law, S. 34.

${ }^{164}$ Ebd., S. 49ff.

165 Cole, Das Selbstbestimmungsrecht indigener Völker, S. 248.
} 
land hinterließen das Vermächtnis der stolen generation. ${ }^{166}$ Auch in Lateinamerika litten Indigene unter einer Gleichheitspolitik der Assimilation und Entwicklung. ${ }^{167}$ Seit der zunehmenden Präsens indigener Gruppen auf internationaler Ebene in den 1970er Jahren änderten sich auch die nationalen Politiken in diesen Teilen der Welt. Meilensteine, die den veränderten Umgang mit indigenen Völkern deutlich machen, waren auch die jeweiligen Entschuldigungen der kanadischen und australischen Prime Ministers für die rassistischen Verbrechen der früheren Assimilationspolitiken in ihren jeweiligen Ländern. ${ }^{168}$ Seit den $1990 \mathrm{er}$ Jahren zeigt sich die Wirkung der globalen Indigenenbewegung auch in Asien und seit der Jahrtausendwende in Afrika. Asiatische und afrikanische indigene Gruppen nutzen die internationalen Foren und das Vokabular des Selbstbestimmungsrechts, um sich so entgegen Marginalisierung und Unterdrückung mit Ansprüchen auf politische Teilhabe zu positionieren. ${ }^{169}$

\section{Rechtsquellen und Institutionen}

Indigene Völker werden in verschiedensten Kontexten auf internationaler Ebene explizit in einer Reihe von Rechtsquellen und Institutionen mit einbezogen: Im internationalen Menschenrechtssystem finden sie als Minderheiten Beachtung. ${ }^{170} \mathrm{Im}$ internationalen Umweltrecht entwickelten sich explizite Regelungen $\mathrm{zu}$ indigenen Völkern, die eine besondere Verbindung dieser Gruppen mit der Natur normieren und Regeln für den Umgang mit traditionellem Wissen aufstel-

166 Bradford W. Morse, „Indigenous Peoples of Canada and Their Efforts to Achieve True Reparations“, in: Federico Lenzerini (Hrsg.), Reparations for Indigenous Peoples: International and Comparative Perspectives, 2008, S. 271-316; Sarah Krakoff und Kristen A. Carpenter, „Repairing Reparations in the American Indian Nation Context“", in: Federico Lenzerini (Hrsg.), Reparations for Indigenous Peoples: International and Comparative Perspectives, 2008, S. 251-70.

${ }^{167}$ Marzia Rosti, „Reparations for Indigenous Peoples in Two Selected Latin American Countries“, in: Federico Lenzerini (Hrsg.), Reparations for Indigenous Peoples: International and Comparative Perspectives, 2008, S. 346.

168 „Ureinwohner in Australien - Historische Entschuldigung“, Süddeutsche Zeitung, 17. Mai 2010; Matthias Rüb, „Harper entschuldigt sich bei Ureinwohnern: Kanada weint nach der historischen Geste“, Frankfurter Allgemeine Zeitung, 6. Dezember 2008.

${ }^{169}$ Benedict Kingsbury, „Indigenous Peoples' in International Law: A Constructivist Approach to the Asian Controversy", American Journal of International Law, Vol. 92, Nr. 3 (1998), S. 414-457; Rachel Murray, ,"The UN Declaration on the Rights of Indigenous Peoples in Africa: The Approach of the Regional Organisation to Indigenous Peoples", in: Steve Allen und Alexandra Xanthaki (Hrsg.), Reflections on the UN Declaration on the Rights of Indigenous Peoples, 2011, S. 485-506.

170 Indigene werden beispielsweise von der Human Rights Commission als Minderheiten im Rahmen von Art. 27 IPbpR behandelt. Siehe dazu „E. I. Minderheitenschutz für indigene Völker und lokale Gemeinschaften“ auf S. 117: Human Rights Committee, „General Comment No. 23: The rights of minorities (Art. 27)“, CCPR/C/21/Rev.1/Add.5, Abs. 3.2, 7; Dieter Kugelmann, „The Protection of Minorities and Indigenous Peoples Respecting Cultural Diversity", Max Planck Yearbook of United Nations Law Online, Vol. 11, Nr. 1 (2007): S. 247, 256. 
len. ${ }^{171}$ Und schließlich spielen sie eine Rolle im Bereich der Rechtsinstrumente geistigen Eigentums, unter denen kulturelle Ausdrucksformen und genetische Ressourcen indigener Völker besonderen Schutz genießen. ${ }^{172}$ Auch wenn all diese Bereiche eigene Entwicklungslinien und Anwendungsbereiche unabhängig von ihrem Einfluss auf die Situation indigener Völker aufweisen, lässt sich keine trennscharfe Unterscheidung in der wechselseitigen Interaktion zwischen der Politik von und für indigene Völker auf der einen Seite und den je eigenen Regelungskontexten des Minderheitenschutzes, des Umweltrechts, und den Instrumenten geistigen Eigentums auf der anderen Seite treffen.

Im Folgenden soll der Wandel der Politik und des Völkerrechts zur Rolle indigener Völker allerdings in seinem Kern, den Entwicklungen bei der Internationalen Arbeitsorganisation und im UN System, nachgezeichnet werden. Der Fokus liegt daher auf den beiden ILO Konventionen Nr. 107 und Nr. 169 und der UNDeklaration zu den Recbten indigener Völker von 2007 sowie in der zunehmend gefestigten Beteiligung im institutionellen Gefüge der Vereinten Nationen.

\section{Convention No. 107 der Internationalen Arbeitsorganisation}

Die Internationale Arbeitsorganisation (ILO) befasste sich mit der Situation der indigenous and tribal populations bereits kurz nach ihrer Gründung 1919.173 Nach ersten Studien 1921 und der aktiven Teilnahme im UN Andean Indian Program verabschiedete sie 1957 die Convention concerning the Protection and Integration of Indigenous and Other Tribal and Semi-Tribal Populations in Independent Countries (Convention No. 107) und damit das erste internationale Instrument mit einem spezifischen Fokus auf die Rechte indigener Völker. ${ }^{174}$ Die Konvention, die bereits in ihrer Entstehung grundlegende Opposition erfuhr und der ILO den Vorwurf der Kompetenzüberschreitung einbrachte, normiert einen umfassenden Kanon an Rechten für indigene Völker. ${ }^{175}$ Sie garantiert ein Diskriminierungsverbot, Landrechte, Arbeitsstandards, den Zugang zu Ausbildungsplätzen, der Sozialversicherung und Gesundheitsfürsorge sowie Zugang zu Bildung. ${ }^{176}$

Zwar proklamiert die Konvention die Achtung vor kulturellen und religiösen Werten und die Zusammenarbeit mit den indigenen Gruppen sowie die Ansicht, dass „populations shall be allowed to retain their own customs and instituti-

\footnotetext{
171 Siehe dazu „C. I. Geschichte der indigenen und lokalen Gemeinschaften im Umweltvölkerrecht“ auf S. 59.

172 Siehe dazu „Teil 4: Verhandlungen im WIPO Intergovernmental Committee“ auf S. 181.

173 Alexandra Xanthaki, Indigenous Rights and United Nations Standards, 2007, S. 49.

174 Internatinal Labour Organization, Convention concerning the Protection and Integration of Indigenous and Other Tribal and Semi-Tribal Populations in Independent Countries (ILO Convention No. 107), 328 UNTS 247, 26. Juni 1957.

175 Xanthaki, Indigenous Rights and United Nations Standards, S. 51.

176 ILO Convention No. 107, Art. 11-26.
} 
ons."177 Dennoch zeigt sich deutlich der Hintergrund und grundlegende Gedanke der Konvention. Indigenous and tribal populations sollen dem Arbeitsmarkt zugeführt, integriert und entwickelt werden. Die eben genannten Elemente der Wertschätzung, Zusammenarbeit und der Bewahrung von Gebräuchen und Institutionen, werden diesen Zielen untergeordnet und nur im Rahmen der nationalen Gesetze und im Sinne dieses integrativen Programms gewährleistet. ${ }^{178}$ Die Assimilationspolitik der Zeit schlägt sich insbesondere in der Zielbestimmung der Konvention in Artikel 2 nieder:

Governments shall have the primary responsibility for developing co-ordinated and systematic action for the protection of the populations concerned and their progressive integration into the life of their respective countries. ${ }^{179}$

Die Bedeutung der Convention No. 107 liegt heute somit vor allem darin, dass sie sich zum ersten Mal mit der Situation indigener Völker befasste und auf internationaler Ebene Rechte und Garantien festschrieb. Sie wird heute in ihrem Schutzgehalt im Lichte ihrer Nachfolgerin Convention No. 169 interpretiert und garantiert in dieser Form einen verbindlichen Schutzstandard für indigene Völker, der die - wenn auch nur wenigen - Ratifikationsstaaten einem regelmäßigen Berichtsverfahren unterwirft. ${ }^{180}$

\section{Convention No. 169 der Internationalen Arbeitsorganisation}

Die Convention No. 169 der ILO, eine überarbeitete und weiterentwickelte Version von Convention No. 107, macht die grundlegende Umkehr von der Assimilationspolitik gegenüber indigenen Völkern deutlich. Vor dem Hintergrund verschiedener Initiativen im Rahmen der UN, wie beispielsweise der Studie des UN Sonderberichterstatters Martinez Cobo zum Problem of Discrimination Against Indigenous Populations ${ }^{181}$, und Entwicklungen auf regionaler Ebene wurde in mehreren Arbeitsschritten die verbindliche Convention Concerning Indigenous and Tribal Peoples in Independent Countries (Convention No. 169) vorbereitet und schließlich 1989 ver-

177 Ebd., Art 4, 5 und 7.

178 Ebd., Art. 7.

${ }^{179}$ Ebd., Art. 2.

${ }^{180}$ ILO Convention No. 107 wurde von 27 überwiegend südamerikanischen Staaten ratifiziert.

Durch die Ratifikation von ILO Convention No. 169 durch wiederum zumeist südamerikanische Staaten erfolgte die automatische Kündigung von neun Vertragsstaaten der ILO Convention No. 107, so dass ihr geographischer Schwerpunkt nunmehr in Afrika und Asien liegt. Sie ist in Kraft für Angola, Bangladesch, Belgien, Kuba, Dominikanische Republik, Ägypten, El Salvador, Ghana, Guinea-Bissau, Haiti, Indien, Irak, Malawi, Pakistan, Panama, Syrien und Tunesien; Xanthaki, Indigenous Rights and United Nations Standards, S. 50, 66.

181 Siehe dazu sogleich unter „B. II. 3. UN Declaration on the Rights of Indigenous Peoples“, auf S. 46, sowie „B. II. Die Definition von José Martínez Cobo“, auf S. 95. 
abschiedet. Bereits 1986 hatte der Governing Body der ILO nach Konsultationen mit dem Meeting of Experts die Überarbeitung der alten Convention No. 107 auf die Agenda gesetzt. ${ }^{182}$ Mit Hilfe eines Berichts des ILO Büros und Umfragen bei den Mitgliedstaaten wurde nach einer ersten General Conference über ein Komitee ein erster Entwurf der Überarbeitung erstellt, welcher nach einer zweiten Runde von Kommentaren durch die Mitgliedstaaten und einer entsprechenden Überarbeitung mit 328 Stimmen, 49 Enthaltungen und einer Gegenstimme angenommen wurde. ${ }^{183}$ Trotz des nachdrücklichen Hinweises des ILO Büros, Mitgliedstaaten mögen indigene Gruppen im Rahmen der Fragebögen auf nationaler Ebene einbeziehen und trotz der Teilnahme von NGO Vertretern als Beobachter während der Verhandlungen zeigten sich die Vertreter indigener Völker mit der Art und dem Umfang ihrer Beteiligung am Überarbeitungsprozess unzufrieden. ${ }^{184}$ Die unzureichende unmittelbare Repräsentation der Gruppen, die von den auf internationaler Ebene erarbeiteten Rechtsinstrumenten letztlich betroffen sind, ist bis heute ein Problem bei Verhandlungen internationaler Organisationen. ${ }^{185}$

Die Garantien der Convention No. 169 fußen in ihrer Struktur auf der Vorgängerkonvention und erweitern diese um soziale und kulturelle Rechte. So sollen Landrechte identifiziert und gesichert werden sowie bei zwingend notwendigen Umsiedlungen der prior informed consent der betroffenen Gruppen eingeholt und entsprechende Entschädigungen in Form von Geld gezahlt werden. ${ }^{186}$ Es sollen unterschiedliche Arbeitsstandards garantiert, Ausbildungen ermöglicht und „Handicrafts, rural and community-based industries, and subsistence economy and traditional activities of the peoples concerned, such as hunting, fishing, trapping and gathering" als wichtige Faktoren der kulturellen und ökonomischen Selbstversorgung und Entwicklung anerkannt werden. ${ }^{187}$ Des Weiteren sollen soziale Sicherungsmechanismen und Bildungseinrichtungen zur Verfügung gestellt, beziehungsweise zusammen mit den Gruppen entwickelt werden, ohne jedoch indigenen Gruppen diese Systeme aufzuzwängen. ${ }^{188}$ Diese Garantien tragen auch einen

\footnotetext{
182 Xanthaki, Indigenous Rights and United Nations Standards, S. 67.

183 Ebd., 68.

${ }^{184}$ Howard R. Berman, „The International Labour Organization and Indigenous Peoples: Revision of ILO Convention No. 107 at the 75th Session of the International Labour Converence, 1988“, The Review of the International Commission of Jurists, Vol. 41, 1988, S. 51.

185 Zur Problematik mangelnder Representation siehe C. IV. Völkerrechtliche Strukturen und Definitionshoheit, auf S. 173; als aktuelles Beispiel kann die rein formelle Beteiligung indigener Gruppen bei den Verhandlungen des WIPO IGC dienen, die aus Protest zwischenzeitlich Unterbrochen wurde, um die Arbeit der Staatenvertreter zu delegimitisieren: A. V. 7. Positionen der Vertreter von Nichtregierungsorganisationen und indigener Völker, auf S. 201.

186 International Labour Organization, Convention concerning Indigenous and Tribal Peoples in Independent Countries (ILO Convention No. 169), 28 ILM 1382, 27. Juni 1989, Art. 13-19.

187 Ebd., Art. 21-23.

188 Ebd., Art. 24-31.
} 
kollektivrechtlichen Charakter: Sie formulieren im Grunde die Ausgestaltung eines allgemeinen Diskriminierungsverbots und verpflichten teilweise $\mathrm{zu}$ positiven Unterstützungsmaßnahmen. ${ }^{189}$ Die Abkehr von der Assimilationspolitik zeigt sich deutlich in den Normen zur consultation and participation indigener Völker und in der Anerkennung ihrer sozialen, wirtschaftlichen und kulturellen Rechte zur Bewahrung ihrer Integrität. ${ }^{190}$ Auch wenn der Konventionstext nicht explizit von Selbstbestimmung spricht, setzt er viele Aspekte der politischen Forderungen indigener Völker in konkrete Garantien um und vollzieht so die Kehrtwende des Politikwechsels „from the vertical and hierarchical narratives of 1957 towards horizontal recognition of an equality-with-difference approach." ${ }^{191}$

Die Konvention profitiert in ihrer Anwendung vom etablierten Berichtsmechanismus der ILO. Danach werden regelmäßige Berichte der Mitgliedstaaten vom Committee of Experts on the Application of Conventions and Recommendations $(C E A C R)$ begutachtet und dialogisch weiter verfolgt. Indigene Völker können Informationen über Veränderungen der Rechtslage und Gerichtsentscheidungen direkt an das CEACR übermitteln sowie in Zusammenarbeit mit Gewerkschaften sogenannte representations einbringen. ${ }^{192}$ Außerdem unterstützt die ILO die Entwicklungen zu den Rechten indigener Völker mit zahlreichen Veröffentlichungen von Berichten, Studien, Arbeitspapieren und Newslettern, um Probleme, Beispiele und best practices aufzuzeigen.

Die Convention No. 169 ist trotz der kleinen Zahl an Ratifikationen und dem geographischen Schwerpunkt in Lateinamerika einer der wichtigsten normativen Bezugspunkte der Entwicklungen zu Rechten indigener Völker. ${ }^{193}$ Der konkrete Normgehalt von Rechten und Garantien setzt die politische Forderung nach Selbstbestimmung in rechtliche Gehalte um und wird in seiner Implementierung durch einen umfassenden Berichtsmechanismus kontrolliert. Von indigenen Völkern wird die Konvention mangels einer umfänglichen Beteiligung ihrer Vertreter im Entstehungsprozess jedoch trotz der positiven Zielrichtung nicht in dem Maße in den politischen Diskurs einbezogen, wie man dies erwarten würde. ${ }^{194}$

\footnotetext{
189 Xanthaki, Indigenous Rights and United Nations Standards, S. 73-75.

${ }^{190}$ ILO Convention No. 169, Art. 2-7; Xanthaki, Indigenous Rights and United Nations Standards, S. 76-80.

191 Patrick Thornberry, „,Who Is Indigenous?", in: Frank Horn (Hrsg.), Economic, Social, and Cultural

Rights of the Sami: International and National Aspects, 1998, S. 17.

192 International Labour Organization, International Labour Organization Constitution, 15 UNTS 40, 28. Juni 1919, Art. 22-24.

193 ILO Convention No. 169 ist in Kraft für 22 Staaten: Argentinien, Bolivien, Brasilien, Zentral Afrikanische Republik, Chile, Kolumbien, Costa Rica, Dänemark, Dominica, Ecuador, Fiji, Guatemala, Honduras, Mexiko, Nepal, Niederlande, Nicaragua, Norwegen, Paraguay, Peru, Spanien und Venezuela.

194 Xanthaki, Indigenous Rights and United Nations Standards, S. 68.
} 


\section{UN Declaration on the Rights of Indigenous Peoples}

Die wichtigste normative Quelle und das Schlüsselinstrument der internationalen Indigenenbewegung ist die am 13. September 2007 von der UN Generalversammlung angenommene United Nations Declaration on the Rights of Indigenous Peoples. ${ }^{195}$ Sie ist das Ergebnis langjähriger Verhandlungen und ein Meilenstein im Kampf indigener Völker für ihr Recht auf Selbstbestimmung. Die Geschichte der Deklaration ist beinahe so lang wie die internationale Indigenenbewegung selbst und bildet heute die Grundlage vieler politischer und rechtlicher Auseinandersetzungen indigener Völker.

1969 befasste sich erstmals ein Organ der Vereinten Nationen explizit mit indigenen Völkern. Das Ergebnis war ein eigenes Kapitel über Schutzmaßnahmen für indigene Völker im Rahmen der Studie zur Racial Discrimination in the Political, Economic, Social and Cultural Spheres, die in der Sub-Commission on the Prevention of Discrimination and Protection of Minorities (Sub-Commission) verhandelt wurde. ${ }^{196}$ Die Sub-Commission schlug 1970 daraufhin vor, eine umfassende Studie zum Problem der Diskriminierung indigener Bevölkerungen in Auftrag zu geben. ${ }^{197}$ Nachdem dieser Vorschlag über die ihr übergeordnete Commission on Human Rights dem wiederum übergeordneten ECOSOC zugeleitet wurde, genehmigte dieser im Mai 1971 die Ausarbeitung einer solchen Studie. ${ }^{198}$ Die Sub-Commission setzte daraufhin José Martinez Cobo als Sonderberichterstatter ein, ${ }^{199}$ der zwischen 1981 und 1984 die umfassende Studie unter dem Titel Study on the Problem of Discrimination against Indigenous Populations zur Situation indigener Völker mit einer Reihe wichtiger Schlussfolgerungen und Empfehlungen veröffentlichte. ${ }^{200}$

195 UN Generalversammlung, „United Nations Declaration on the Rights of Indigenous Peoples“, UN Doc. A/Res/61/295, 13. September 2007; Erica-Irene Daes, „,The UN Declaration on the Rights of Indigenous Peoples: Background and Appraisal“, S. 11, 38.

196 Sub-Commission on the Prevention of Discrimination and Protection of Minorities, Mr. Hernán Santa Cruz, „Special Study of Racial Discrimination in the Political, Economic, Social and Cultural Spheres“, UN Doc. E/CN.4/Sub.2/301, 24. Juni 1969, Kapitel 8.

197 Augusto Willemsen Diaz, „How Indigenous Peoples’ Rights reached the UN“, in: Claire Charters und Rodolfo Stavenhagen (Hrsg.), Making the Declaration Work, the United Nations Declaration on the Rights of Indigenous Peoples, 2009, S. 22.

198 Economic and Social Council, „1589 (L) The problem of indigenous populations“, UN Doc. E/5044, 21. Mai 1971.

199 Sub-Commission on the Prevention of Discrimination and Protection of Minorities, ,Report of the Twenty-Fourth Session of the Sub-Commission on the Prevention of Discrimination and Protection of Minorities to the Commission on Human Rights, Resolution 8 (XXIV)“, UN Doc. E/CN.4/Sub.2/323, 6. Oktober 1971, S. 59.

${ }^{200}$ Erica-Irene Daes, „The UN Declaration on the Rights of Indigenous Peoples: Background and Appraisal“, S. 12; Sub-Commission on the Prevention of Discrimination and Protection of Minorities, José R. Martínez Cobo, „Study of the Problem of Discrimination against Indigenous Populations“, einzelne Berichte zwischen 1981-1983, zusammengefasst neu veröffentlich unter: UN Doc. E/CN.4/Sub.2/1986/7 und Add.1-4. 
Bereits 1981 regte die Sub-Commission beim ECOSOC die Gründung der Working Group on Indigenous Populations (WGIP) an, die nach seiner Zustimmung im Jahr 1982 noch im August desselben Jahres zu ihrer ersten Sitzung zusammenkam. Die WGIP, die von 1984 bis 2001 unter dem Vorsitz von Erica-Irene Daes geführt wurde, hatte die Aufgabe

(1) to review national developments pertaining to the promotion and protection of the human rights and fundamental freedoms of indigenous population [...and]

(2) to give special attention to the evolution of standards concerning the rights of indigenous populations..$^{201}$

Sie besteht aus fünf unabhängigen Experten der Sub-Commission und versammelt in ihren jährlichen Sitzungen eine Vielzahl von Vertretern indigener Völker und ihrer Organisationen sowie Regierungsvertreter, Nichtregierungsorganisationen und Vertreter von UN Organen. In Bezug auf die Entwicklung internationaler Standards wurden bereits 1984 erste Prinzipien für die Entwicklung einer Deklaration zusammengestellt, die im folgenden Jahr durch einen weiteren Entwurf von Prinzipien ergänzt und schließlich intensiv während der vierten Sitzung der WGIP diskutiert wurden, so dass 1985 der Entwurf von sieben Prinzipien dem ECOSOC übersandt wurden. ${ }^{202}$

Von 1987 bis 1993 erarbeitete die WGIP, der diesbezüglichen Empfehlung des ECOSOC folgend, einen Entwurf der Deklaration der Rechte indigener Völker. Mit der Perspektive einer globalen Anwendbarkeit und dem Ziel, umfassende Zustimmung von Seiten der Staaten zu erreichen, wurden die komplexen Themen der kollektivrechtlichen Ausgestaltung, Fragen der Autonomie sowie neue Konzepte, wie das Recht auf Entwicklung, verhandelt. Ausgehend von den sieben Prinzipien legte Erica-Irene Daes 1988 einen ersten Entwurf einer Deklaration vor, der das kollektive Recht auf Existenz, einen Gleichheitsgrundsatz, kulturelle Rechte, wirtschaftliche Rechte mit einer besonderer Betonung von Landrechten sowie politische Rechte vorsah. ${ }^{203}$ Insbesondere der Aspekt der politischen Rechte wurde lange strittig diskutiert, da er durch die Verknüpfungen mit der Frage der Bezeichnung als ,Völker ${ }^{6}$ und dem Konzept der Selbstbestimmung grundlegende Kontroversen auslöste. Von Seiten der indigenen Völker ist dieser Aspekt der Kerngehalt ihres Selbstverständnisses. Sie verstehen sich als ,Völker ${ }^{6}$ im Sinne von Artikel 1 IPbpR, dem konstitutiven Element der völkerrechtlichen Rechtssubjek-

${ }^{201}$ Economic and Social Council, „1982/34 Study of the Problem of Discrimination against Indigenous Populations“, E/1982/82, 7. Mai 1982; Siegfried Wiessner, „United Nations Declaration on the Rights of Indigenous Peoples, General Assembly resolution 61/295“, Audiovisual Library of the United Nations, 2009.

202 Daes, „The UN Declaration on the Rights of Indigenous Peoples: Background and Appraisal“, S. 12.

${ }^{203}$ Ebd., S. 26. 
tivität und somit den Staaten ebenbürtig. Von Seiten der Regierungen wurde die Bezeichnung als Volk und die Proklamation des Rechts auf Selbstbestimmung aus Ängsten vor Sezession indigener Völker vehement zurückgewiesen.204 Mit der Zeit konkretisierten die Verhandlungen in der WGIP die Balance zwischen dem Selbstbestimmungsrecht indigener Völker als die konzeptuelle Grundlage ihres Selbstverständnisses und der Aussage, dass dies keinen Automatismus einer Sezession impliziert.

Nach langjährigen Verhandlungen zur Klärung der komplexen Zusammenhänge des Anspruchs auf Selbstbestimmung und der Bezeichnung als, Volk' sowie den damit zusammenhängenden Landrechten und Ansprüchen auf interne und externe Autonomie wurde 1994 ein erster Entwurf der Deklaration nach Zustimmung der Sub-Commission der Commission on Human Rights zugeleitet. Diese setzte eine open-ended inter-sessional Working Group unter dem Vorsitz von Luis-Enrique Savez ein, um den Entwurf der Deklaration zu vervollkommnen. Aufgrund der Verzögerung durch einige Staaten und Vertreter indigener Völker dauerte es bis ins Jahr 2006, dass ein Entwurf angenommen und dem Human Rights Council vorgelegt wurde. ${ }^{205}$ Nach ausführlichen Diskussionen im Human Rights Council wurde der Entwurf dem Third Committee der UN Generalversammlung zugeleitet, welches wiederum Änderungen einarbeitete und entschied, weitere Erörterungen und Maßnahmen aufzuschieben. ${ }^{206}$ Nachdem 67 Mitgliedstaaten die Präsidentin der Generalversammlung Sheikha Haya Rashed Al Khalifa darum baten, keine neuen, ergebnisoffenen Verhandlungen zu beginnen, wurden durch informelle Vermittlung von Hilario G Davide Jr. nur noch gezielt letzte Änderungen diskutiert. Bis zu den letzten Tagen vor der Abstimmung wurden dabei Änderungen und Kompromisse insbesondere für die afrikanische Gruppe berücksichtigt. ${ }^{207}$

Die Deklaration wurde schließlich mit einer überwältigenden Mehrheit von 143 Stimmen, elf Enthaltungen und den vier Gegenstimmen Australiens, Kanadas, Neuseelands und der USA angenommen. Diese vier Staaten begründeten ihre ablehnende Haltung hauptsächlich mit möglichen rechtlichen Komplikation ihres jeweiligen Verfassungsrechts und in Bezug auf Ansprüche Dritter entgegen den Garantien indigener Landrechte. Später haben sie jedoch ihre Zustimmung zur

${ }^{204}$ Karen Engle, „On Fragile Architecture: The UN Declaration on the Rights of Indigenous Peoples in the Context of Human Rights“, European journal of international law, Vol. 22, Nr. 1 (2011), S. 144.

${ }^{205}$ Daes, „The UN Declaration on the Rights of Indigenous Peoples: Background and Appraisal“, S. 34 .

206 UN Generalversammlung, ,Working group of the Commission on Human Rights to elaborate a draft declaration in accordance with paragraph 5 of General Assembly resolution 49/214 of 23 December 1994“, UN Doc. A/Res/61/178, 6. März 2007.

${ }^{207}$ Daes, „The UN Declaration on the Rights of Indigenous Peoples: Background and Appraisal“, S. 36 . 
Deklaration erklärt. ${ }^{208}$ Trotz ihres rechtlich unverbindlichen Charakters als Resolution der UN Generalversammlung bildet die Deklaration heute den normativen Kerngehalt der globalen Indigenenbewegung und fungiert somit als Schlüsselargument in politischen und rechtlichen Auseinandersetzungen.209

\section{Institutionen}

Neben den bereits genannten Institutionen der WGIP, der open-ended inter-sessional Working Group und des Sonderberichterstatters José Martínez Cobo der Study of the Problem of Discrimination Against Indigenous Populations, die ihre Arbeit bis heute beendet haben oder institutionell umgewandelt wurden, befassen sich aktuell verschiedene Akteure des UN Menschenrechtssystems mit der Situation indigener Völker. Die 2006 grundlegend reformierte und teilweise umstrukturierte Architektur der UN Menschenrechtsinstitutionen wird vom Human Rights Council (vormals Human Rights Commission) getragen. Der Human Rights Council besteht aus 47 von der UN Generalversammlung gewählten Staatenvertretern. Unter seiner Ägide stehen der Special Rapporteur on the Situation of Human Rights and Fundamental Freedoms of Indigenous People - dieses Amt bekleidete von 2001 bis 2008 Rodolfo Stavenhagen und seit 2008 James Anaya - sowie der UN Expert Mechanism on the Rights of Indigenous Peoples (vormals die Working Group on Indigenous Populations), ein Gremium bestehend aus fünf unabhängigen Experten.

Die ehemalige Human Rights Sub-Commission on the Promotion and Protection of Human Rights (bis 1999 Sub-Commission on Prevention of Discrimination and Protection of Minorities), in der die Entwicklungen ihren Anfang nahmen, wurde 2006 durch das Advisory Committee ersetzt, welches sich umfassend mit Fragen des internationalen Menschenrechtsschutzes und nicht nur mit der Situation indigener Völker befasst.

Im Mai 2002 tagte erstmals das UN Permanent Forum on Indigenous Issues (UNPFII), welches zwar dem ECOSOC als beratendes Gremium zugeordnet, aber grundsätzlich eine von Indigenen getragene Institution ist. Es hat folgende Aufgaben:

- provide expert advice and recommendations on indigenous issues to the Council, as well as to programmes, funds and agencies of the United Nations, through the Council

\footnotetext{
208 Engle, „On Fragile Architecture“, S. 145.

${ }^{209}$ Grand Chief Ed John, ,Remarks by the Chair of the United Nations Permanent Forum on Indigenous Issues", High-Level Event to Commemorate the 5th Anniversary of the Adoption of the UN Declaration on the Rights of Indigenous Peoples, 17. Mai 2012; Rodolfo Stavenhagen, "Making the Declaration Work“, in: Claire Charters und Rodolfo Stavenhagen (Hrsg.), Making the Declaration Work, the United Nations Declaration on the Rights of Indigenous Peoples, 2009, S. 352-371.
} 
- raise awareness and promote the integration and coordination of activities related to indigenous issues within the UN system

- prepare and disseminate information on indigenous issues. ${ }^{210}$

Das UNPFII besteht aus 16 Experten und einer jährlich stattfindenden, zweiwöchigen Tagung mit Vertretern von indigenen Völkern und ihren Organisationen sowie Vertretern von Regierungen, internationalen Organisationen und weiteren Nichtregierungsorganisationen. ${ }^{211}$ Für die Vertretung und Präsenz indigener Völker bei den Vereinten Nationen ist das UNPFII von zentraler Bedeutung, da Indigene hier als eigenständige Gemeinschaften anerkannt werden und Zugang zu internationalen Entscheidungsfindungsprozessen erlangen ohne, wie sonst üblich, als Interessenvertreter in thematisch festgelegten Verhandlungen inhaltlich eingeschränkt zu werden. ${ }^{212}$

\section{Zwischenergebnis: eine Kehrtwende der Indigenitätspolitik}

Im Zuge einer beispiellosen internationalen politischen Bewegung haben sich indigene Völker als aktive Subjekte im institutionellen Gefüge internationaler Organisationen etabliert und es geschafft, ihren Anspruch auf Selbstbestimmung in völkerrechtlichen Instrumenten zu verankern. Das Ergebnis der internationalen Indigenenbewegung spiegelt sich in den Zielrichtungen der internationalrechtlichen Quellen wider: Es zeigt die Kehrtwende von der historisch lange vorherrschenden Assimilationspolitik, unter der indigene Völker marginalisiert und unterdrückt wurden, hin zu einer Politik der Wertschätzung und Bewahrung der kulturellen Integrität dieser Gruppen.

III. Das Selbstbestimmungsrecht indigener Völker

Die Kernforderung der internationalen Indigenenbewegung ist der Anspruch auf das Selbstbestimmungsrecht indigener Völker. Die Idee des Konzepts ,Selbstbestimmung' fußt auf den Entwicklungen der amerikanischen Unabhängigkeitsbewegung und der Französischen Revolution. ${ }^{213}$ Es wurde geprägt durch die nationalstaatlichen Ideen des 19. Jahrhunderts sowie die sozialistischen und kommunistischen Gedanken des frühen 20. Jahrhunderts. ${ }^{214}$ Seine völkerrechtliche Bedeutung erhielt das Konzept im 14-Punkte-Programm des amerikani-

210 Economic and Social Council, „2000/22 Establishment of a Permanent Forum on Indigenous Issues“, UN Doc. E/2000/Inf/2/Add.2, 15. August 2000, Resolution 2000/22, Abs. 2.

211 UN Permanent Forum on Indigenous Issues, „Handbook for Participants“, 2007, S. 12.

212 Joshua Castellino, „Indigenous Rights and the Right to Development: Emerging Synergies or Collusion?", in: Steve Allen und Alexandra Xanthaki (Hrsg.), Reflections on the UN Declaration on the Rights of Indigenous Peoples, 2011, S. 383; Xanthaki, Indigenous Rights and United Nations Standards, S. 4.

213 Daniel Thürer, Das Selbstbestimmungsrecht der Völker, 1976, S. 15-19.

214 Cole, Das Selbstbestimmungsrecht indigener Völker, S. 15-17. 
schen Präsidenten Woodrow Wilson zur Neuordnung Europas nach dem Ersten Weltkrieg. ${ }^{215}$ Und schließlich wurde es durch die Verankerung in der Charta der Vereinten Nationen zu einem der bedeutendsten Konzepte des modernen Völkerrechts. 216

Das Selbstbestimmungsrecht der Völker, welches in den Zielbestimmungen der UN-Charta in Artikel 1(2) niedergelegt ist, wandelte seine Bedeutung vom Konzept der friedlichen Beziehung souveräner Staaten zur argumentativen Grundlage der Dekolonisation. ${ }^{217}$ Dabei beschränkte sich das Verständnis insbesondere in der Anwendung der ,Dritte Welt Staaten' auf den Aspekt der externen Selbstbestimmung und berücksichtigte nicht die Rechte von Minderheiten oder sonstigen substaatlichen Gruppen.218 Das Selbstbestimmungsrecht der Völker unterlag im Prozess der Dekolonisation außerdem der salt-water-doctrine, die seine Anwendung auf die Überseegebiete der europäischen Kolonien beschränkte, sowie dem Grundsatz uti possidetis, ita possideatis, nach dem die zumeist willkürlich gezogenen Kolonialgrenzen gewahrt wurden. ${ }^{219}$ Schließlich fand es Eingang in die beiden internationalen Menschenrechtspakte, wodurch der Bedeutungsgehalt der demokratischen Herrschaftsbestimmung durch das Volk, das internationale Nichteinmischungsgebot und die Hoheit über natürliche Ressourcen impliziert wurden. ${ }^{220}$ Die Normierung des Selbstbestimmungsrechts in den Menschenrechtspakten sowie seine Konkretisierung in der Friendly Relations Declaration von 1970 prägten die globale Anwendbarkeit dieses Rechts und erweiterten seine Bedeutung über den Kontext der Dekolonisation hinaus. ${ }^{221}$ Es hat insbesondere im Kontext der globalen Indigenenbewegung aktuelle Brisanz gewonnen, da indigene Völker durch die Selbstidentifikation als people die Implikationen des Selbstbestimmungsrechts der Völker für sich nutzbar machten. Das Selbstbestimmungsrecht der Völker, wie es in der Deklaration über die Rechte indigener Völker von 2007 niedergelegt wurde, umfasst politische Rechte, Landrechte, kulturelle und soziale Rechte sowie das Recht auf Entwicklung.

\footnotetext{
215 Joshua Castellino, International law and self-determination, 2000, S. 13-19.

${ }^{216}$ Cole, Das Selbstbestimmungsrecht indigener Völker, S. 24.

217 Antonio Cassese, Self-determination of peoples, 1996, S. 44.

218 Ebd., S. 46.

219 James Crawford, The creation of states in international law, 2006, S. 611; Castellino, International law and self-determination, S. 109-44.

${ }^{220}$ Cassese, Self-determination of peoples, S. $65 \mathrm{f}$.

${ }^{221}$ Cole, Das Selbstbestimmungsrecht indigener Völker, S. 26-31.
} 
B. Die Rechte indigener Völker

\section{Politische Rechte}

Der Kern des Selbstbestimmungsrechts proklamiert die politischen Rechte der Autonomie und der eigenen Regierung eines Volkes. Die Deklaration normiert das Selbstbestimmungsrecht in Artikel 3 und führt es in Artikel 4 weiter aus. Darin heißt es:

\section{Article 3 \\ Indigenous peoples have the right to self-determination. By virtue of that right they freely determine their political status and freely pursue their economic, social and cultural development. \\ Article 4 \\ Indigenous peoples, in exercising their right to self- determination, have the right to autonomy or self-government in matters relating to their internal and local affairs, as well as ways and means for financing their autonomous functions. ${ }^{222}$}

Selbstbestimmung bedeutet, in eigenen Angelegenheiten nach innen und außen als Volk selbst entscheiden zu dürfen und umfasst auch die wichtige Kompetenz der Finanzhoheit. Dies schließt sowohl die grundlegende Gestaltung der eigenen Regierungsstrukturen als auch die fortwährenden politischen Entscheidungen mit ein, die auf eine kollektive Entscheidung des Volkes zurückgeführt werden können muss. ${ }^{223}$

Die Unterscheidung zwischen interner und externer Selbstbestimmung, wie sie noch im Prozess der Dekolonisation prägend war, ist hier mangels einer formellen Grenzziehung zwischen den ,Völkern' nicht möglich.

Given the reality of multiple human associational patterns in today's world, including but not exclusively those organized around the state, it is distorting to attempt to organize selfdetermination precepts into discrete internal versus external spheres defined by reference to presumptively mutually exclusive peoples. ${ }^{224}$

In Anbetracht moderner Entwicklungen von multi- und interkulturellen Gesellschaften wäre solch eine Trennung praktisch nur schwer umzusetzen und in Anbetracht der Komplexität kultureller Identitäten realitätsfern.

Das Recht auf Selbstbestimmung indigener Völker impliziert vor diesem Hintergrund auch nicht das Recht auf Sezession und Staatsgründung. Dieser Aspekt war einer der schwierigsten Streitpunkte bei der Erarbeitung der Deklaration, der

\footnotetext{
222 UN Generalversammlung, „United Nations Declaration on the Rights of Indigenous Peoples“, UN Doc. A/Res/61/295, Art. 3 und 4.

223 Anaya, Indigenous peoples in international law, S. 104-106.

${ }^{224}$ Ebd., S. 105.
} 
bis zuletzt verhandelt wurde. Entgegen den Versuchen Artikel 3 zur Selbstbestimmung zu ändern, wurde in Artikel 46 explizit deutlich gemacht, dass „nothing in this declaration may be interpreted as [...] authorizing [...] any action which would dismember or impair [...] the territorial integrity or political unity of sovereign and independent States. “225 Dennoch wird vertreten, dass die Sezession vom Heimatstaat im Einzelfall für alle beteiligten Gruppen die bessere Lösung darstellen oder Abhilfe schaffen könne, wenn substantielle Rechte nicht auf sonstigem Wege garantiert werden können.226

Der politische Aspekt des Selbstbestimmungsrechts, wie er in der Deklaration und auch schon in der ILO Convention No. 169 verankert ist, zielt insgesamt vielmehr auf die Integration indigener Völker in den politischen Diskurs im Rahmen des Heimatstaates ab und sieht Mitbestimmungs- und Beteiligungsrechte für Entscheidungen vor, welche die indigenen Gruppen betreffen.227 Für die eigenen politischen Diskurse, die Interaktion gegenüber der Politik des Heimatstaates und auch für die Beteiligung im politischen Prozess des Staates sollen die Institutionen indigener Völker erhalten und gestärkt werden.228

\section{Landrechte}

Bereits die frühe ILO Convention No. 107 normierte Landrechte für indigene Völker, indem sie die hergebrachten Regeln der Nutzung und Übertragung von Land anerkannte und zwangsweise Umsiedelungen nur bei free consent oder aus Gründen der nationalen Sicherheit, öffentlichen Gesundheit oder der ,Entwicklung' sowie mit Entschädigung durch vergleichbares Land zuließ. ${ }^{229}$ Convention No. 169 erweiterte den Schutz der Landrechte auf die gesamte „environment of the areas which the peoples concerned occupy or otherwise use. “230 Sie entzog den Staaten die Berechtigung zur Enteignung aus den soeben genannten Gründen und normierte vielmehr die Pflicht, Maßnahmen zu ergreifen, welche den indigenen Völkern die Nutzung ihres traditionell genutzten Landes gestattete. ${ }^{231}$ Somit impliziert Convention No. 169 das Recht auf die lebenden Ressourcen des

\footnotetext{
225 UN Generalversammlung, „United Nations Declaration on the Rights of Indigenous Peoples“, UN Doc. A/Res/61/295, Art. 46; Daes, „The UN Declaration on the Rights of Indigenous Peoples: Background and Appraisal", S. 36f.

226 Anaya, Indigenous peoples in international law, S. 109; Xanthaki, Indigenous Rights and United Nations Standards, S. $168 \mathrm{f}$.

227 Anaya, Indigenous peoples in international law, S. 153-156; ILO Convention No. 169, Art. 6; UN Generalversammlung, „United Nations Declaration on the Rights of Indigenous Peoples“, UN Doc. A/Res/61/295, Art. 18, 19; Barsh, „Indigenous Peoples“, S. 842f.

228 UN Generalversammlung, „United Nations Declaration on the Rights of Indigenous Peoples“, UN Doc. A/Res/61/295, Art. 5, 35-37.

229 ILO Convention No. 107, Art. 11-14.

${ }^{230}$ ILO Convention No. 169, Art. 13 (2).

231 Ebd., Art. 14.
} 
B. Die Rechte indigener Völker

Landes und erkennt die Institutionen indigener Völker zur Kontrolle und Nutzung des Landes an. ${ }^{232}$

Die UNDRIPs erweitert den Rechtsumfang der Landrechte auf natürliche Ressourcen wie Mineralien und Öl und formuliert in ihrem Wortlaut noch deutlicher auch einen rückwirkenden Anspruch auf Land, welches sie traditionell besaßen, bewohnten oder sonst nutzten oder sich aneigneten. ${ }^{233}$

Indigenous peoples have the right to the lands, territories and resources which they have traditionally owned, occupied or otherwise used or acquired. 234

Die UNDRIPs normiert die Rechte auf Land, Territorien und Ressourcen, das Recht nicht umgesiedelt zu werden, Ansprüche auf Wiedergutmachung und Entschädigung, die Anerkennung indigener Pachtverhältnisse sowie den Schutz und die Pflege der Umwelt und Fruchtbarkeit des Landes. ${ }^{235}$ Für die Umsetzung dieser Rechte bedarf es unabhängiger Institutionen, welche über die Ansprüche indigener Völker auf ihr Land entscheiden und somit Rechtssicherheit schaffen sollen. ${ }^{236}$

\section{Kulturelle und soriale Rechte}

Die Garantien kultureller und sozialer Rechte finden sich explizit in zahlreichen Artikeln der UNDRIPs und durchdringen den normativen Gehalt dieses Dokuments in jeglicher Hinsicht. ${ }^{237}$ Vor dem Hintergrund der historischen Assimilationspolitik erschließt sich die besondere Bedeutung des Rechts „, to live in freedom, peace and security as distinct peoples and [...] not be subjected to any act of genocide or any other act of violence, including forcibly removing children of the group to another group" sowie der Garantie gegen Assimilation und die Zerstörung der Kultur. ${ }^{238}$ Die Deklaration normiert weiterhin die Rechte auf

\footnotetext{
232 Barsh, „Indigenous Peoples“, S. 846.

233 Jérémie Gilbert und Cathal Doyle, „A New Dawn over the Land: Shedding Light on Collective Ownership and Consent", in: Steve Allen und Alexandra Xanthaki (Hrsg.), Reflections on the UN Declaration on the Rights of Indigenous Peoples, 2011, S. 297-301.

${ }^{234}$ UN Generalversammlung, „United Nations Declaration on the Rights of Indigenous Peoples“, UN Doc. A/Res/61/295, Art. 26 (1).

235 Ebd., Art. 8 (2) b), 10, 25-31.

236 Sub-Commission on the Prevention of Discrimination and Protection of Minorities, Erica-Irene Daes, „Indigenous peoples and their relationship to land“, Final working paper prepared by the Special Rapporteur, Mrs. Erica-Irene A. Daes, UN Doc. E/CN.4/Sub.2/2001/21, 11. Juni 2001, Abs. 152-158.

${ }^{237}$ Elsa Stamatopoulou, „Taking Cultural Rights Seriously: The Vision of the UN Declaration on the Rights of Indigenous Peoples“, in: Steve Allen und Alexandra Xanthaki (Hrsg.), Reflections on the UN Declaration on the Rights of Indigenous Peoples, 2011, S. 392.

238 UN Generalversammlung, „United Nations Declaration on the Rights of Indigenous Peoples“, UN Doc. A/Res/61/295, Art. 7 (2) und 8.
} 
Zugehörigkeit zu einer indigenen Gemeinschaft, auf Ausübung kultureller Traditionen und Gebräuche, den Erhalt und die Entwicklung historischer Stätten, Artefakte und Zeremonien sowie die Rückführung kulturellen, geistigen, religiösen und spirituellen Eigentums. ${ }^{239}$ Sie garantiert Rechte auf Bildung, Sprache, Medien und Gesundheit, sodass diese Bereiche im Einklang mit den Traditionen, Gebräuchen und Praxen indigener Völker ausgeübt und weiterentwickelt werden können. ${ }^{240}$ Außerdem wird ihr Recht auf Erhalt, Kontrolle, Schutz und Entwicklung ihres kulturellen Erbes, ihres traditionellen Wissens und traditioneller kultureller Ausdrucksformen und auf ihre Wissenschaft, Technologien und Kultur, einschließlich menschlicher, tierischer und pflanzlicher genetischer Ressourcen garantiert sowie entsprechende Rechte geistigen Eigentums anerkannt. ${ }^{241}$ Die Deklaration normiert somit einen umfassenden Schutz der kulturellen Integrität indigener Völker, einschließlich ihrer kulturellen Objekte, Institutionen und immateriellen Werte.

\section{Recht auf Entwicklung}

Das Recht auf Entwicklung, welches in der UNDRIPs sowohl explizit ausgeführt ist und sich ebenso implizit an verschiedenen Stellen zeigt, ${ }^{242}$ begründet sich, wie auch die überkommenen Politiken der Assimilation und ,Entwicklung', durch die ökonomisch nachteilige Situation indigener Völker. Hintergrund sind die Ausbeutung der natürlichen Ressourcen des Landes indigener Völker und die diskriminierende Ausgrenzung aus sozialstaatlichen Strukturen. ${ }^{243}$ Das Recht auf Entwicklung wird hier jedoch im Lichte des Selbstbestimmungsrechts als das Recht auf die eigene Entscheidung über die Art und Weise der Entwicklung fokussiert.

In den Diskursen zum Recht auf Entwicklung werden Verknüpfungen zu den normativen Instrumenten der sogenannten ,Dritten Generation der Menschenrechte' mit dem kollektiven Recht auf Entwicklung, den Arbeiten der Vereinten Nationen zu den Millennium Development Goals, den Projekten des UNDP und UNICEF sowie zu Entwicklungen im internationalen Umweltrecht hergestellt.244 Diese Verknüpfungen zeichnen das Bild des ethnodevelopment oder alternative development, welches den Einklang sozio-kultureller Besonderheiten, der ökologisch

\footnotetext{
239 Ebd., Art. 9-12.

240 Ebd., Art. 13-16, 24.

241 Ebd., Art. 31; Rosemary J. Coombe, The cultural life of intellectual properties: authorship, appropriation and the law, 1998.

242 Castellino, „Indigenous Rights and the Right to Development: Emerging Synergies or Collusion?", S. 378-380.

243 Anaya, Indigenous peoples in international law, S. 149.

244 Castellino, , Indigenous Rights and the Right to Development: Emerging Synergies or Collusion?", S. 372-377, 385; Anaya, Indigenous peoples in international law, S. 148-150.
} 
B. Die Rechte indigener Völker

nachhaltigen Entwicklung und Beteiligung indigener Gemeinschaften herstellt. ${ }^{245}$ Das Recht auf Entwicklung indigener Völker ist dabei jedoch nicht auf das romantisierte Bild der subsistenzwirtschaftlichen Nutzung natürlicher Ressourcen in Kleinstbetrieben und Handarbeit beschränkt, sondern eröffnet gerade die Entscheidungsfreiheit, eigene Wege der ökonomischen Entwicklung zu gehen. ${ }^{246}$

\section{Zusammenfassung}

Das Selbstbestimmungsrecht indigener Völker, wie es nun in der UNDRIPs verankert ist, macht die Kehrtwende weg von der Assimilationspolitik hin zur Anerkennung der Existenz indigener Völker in Form von Wertschätzung und einer Politik der Beteiligung deutlich. ${ }^{247}$ Die UNDRIPs knüpft somit an den Geist des Schutzes der kulturellen Integrität an, wie er auch im Minderheitenschutz des Artikel $27 \mathrm{IPbpR}$ und der International Convention on the Elimination of All Forms of Racial Discrimination (CERD) bereits verankert wurde und in Bezug auf indigene Völker Anwendung findet. ${ }^{248}$ Explizit ausgeschlossen in der Ausgestaltung des Selbstbestimmungsrechts der UNDRIPs ist in Artikel 46 das Recht auf Sezession und konstituiert das Selbstbestimmungsrecht indigener Völker also nicht in derselben Form wie die Charta der Vereinten Nationen dies für Staatsvölker tut. Der Anspruch politischer Selbstbestimmung indigener Völker findet seine Anwendung demnach im Rahmen der bestehenden Ländergrenzen, was insbesondere den Sorgen der afrikanischen Staaten Rechnung trägt und der Einordnung der Deklaration in den Kontext der internationalen Menschenrechte Ausdruck verleiht. Die Herausforderungen liegen nun in der Um- und Durchsetzung der in der Deklaration niedergelegten Rechte.

\section{Implementierung und Verfahren}

Die internationalen Rechte indigener Völker werden auf nationaler Ebene jeweils durch Ratifikation und durch die Anwendung von Völkergewohnheitsrecht implementiert, soweit die internationalen normativen Quellen eine Rechtsüberzeugung ausdrücken und durch Staatenpraxis Bestätigung erfahren. ${ }^{249}$

245 Jan Nederveen Pieterse, „My Paradigm or Yours? Alternative Development, Post-Development, Reflexive Development", Development and change, Vol. 29, Nr. 2 (1998), S. 347.

246 Pieterse, „My Paradigm or Yours?“; Anthony Bebbington, „Modernization from Below: An Alternative Indigenous Development?", Economic geography, Vol. 69, Nr. 3 (1993), S. 274-292; Karen Engle, The elusive promise of indigenous development: rights, culture, strategy, 2010, S. 183-221.

247 Dies wird besonders in Art. 8 deutlich. UN Generalversammlung, „United Nations Declaration on the Rights of Indigenous Peoples“, UN Doc. A/Res/61/295, Art. 8.

248 Patrick Thornberry, „Integrating the UN Declaration on the Rights of Indigenous Peoples into CERD Practice", in: Steve Allen und Alexandra Xanthaki (Hrsg.), Reflections on the UN Declaration on the Rights of Indigenous Peoples, 2011, S. 61-92.

${ }^{249}$ Einen Überblick der Implementierung der Rechte indigener Völker auf nationaler Ebene findet sich bei: Anaya, Indigenous peoples in international law, S. 185ff. 
Auf internationaler Ebene sieht die UNDRIPs keine eigenen Durchsetzungs-, Berichts- oder Beschwerdeverfahren vor. Jedoch wirkt sie als Teil der internationalen Menschenrechtsarchitektur auf die normativen Werte des Minderheitenschutzes, das Gebot der Nichtdiskriminierung und weitere Menschenrechte mit Bedeutung für indigene Völker ein und konkretisiert diese bei der Anwendung und Auslegung in einschlägigen Verfahren der bestehenden Institutionen. Insofern bieten die etablierten Verfahren im Rahmen des globalen und regionalen Menschenrechtsschutzes sowie auch nationale Institutionen einen Zugang zur Durchsetzung der Garantien der UNDRIPs. Die Convention No. 169 profitiert von den etablierten Strukturen der ILO, die ihr zur Umsetzung verhelfen. Zusätzlich finden sich weitere Institutionen speziell für die Situation indigener Völker.

\section{Berichts- und Überwachungsverfahren}

Im Rahmen der Vereinten Nationen befassen sich das Permanent Forum on Indigenous Issues, der Special Rapporteur on the Situation of Human Rights and Fundamental Freedoms of Indigenous People sowie der UN Expert Mechanism on the Rights of Indigenous Peoples mit der Situation indigener Völker. Diese legen ihrer Arbeit die UNDRIPs als Bewertungsmaßstab zu Grunde. Die Garantien der Convention No. 169 sind Gegenstand des Berichtsverfahrens der ILO. ${ }^{250}$ Im Rahmen der internationalen Menschenrechtsverträge bestehen jeweils Überwachungsmechanismen, die Menschenrechtsverletzungen auch gegenüber indigenen Völkern thematisieren. Insbesondere das Human Rights Committee des IPbpR und das Committee on the Elimination of Racial Discrimination der CERD garantieren durch ihre Besetzung mit Experten eine eingehende Überwachung der Umsetzung entsprechender Rechte. ${ }^{251}$ Außerdem hat sich auf regionaler Ebene die InterAmerican Commission on Human Rights durch gesonderte Berichte zur Menschenrechtssituation in den $O A S$ Staaten etabliert. ${ }^{252}$

\section{Beschwerdeverfahren}

Für individuelle Beschwerdeverfahren stehen auf globaler Ebene das Human Rights Committee sowie die Institutionen der ILO bereit, die im Rahmen des internationalen Minderheitenschutzes beziehungsweise der Convention No. 169 einzelne Fälle begutachten. ${ }^{253}$ Auf regionaler Ebene bieten sich Möglichkeiten, Beschwerden bei den jeweiligen Menschenrechtsgerichtshöfen einzureichen, die

\footnotetext{
250 Siehe dazu bereits oben „B. II. 2. Convention No. 169“, auf S. 43.

251 Anaya, Indigenous peoples in international law, S. 228-232.

252 Ebd., S. 232-234.

253 Zum Verfahren vor dem Human Rights Committee im Rahmen des internationalen Minderheitenschutzes siehe: „A. IV. 2. Berichts- und Beschwerdemechanismen unter dem IPbpR“, auf S. 33.
} 
B. Die Rechte indigener Völker

im Kontext individueller Menschenrechtsgarantien indirekt die Wertungen der UNDRIPs und entsprechende Rechte indigener Völker einbeziehen können. In Amerika nimmt außerdem die Inter-American Commission on Human Rights Petitionen zu Verletzungen entgegen. ${ }^{254}$ Schließlich ergeben sich auf nationaler Ebene unterschiedliche Möglichkeiten, die Deklaration argumentativ und je nach nationaler Verbindlichkeit und Umsetzung einzubringen. 255

\section{Zusammenfassung: globale politische Bewegung für Selbstbestimmung}

Die globale Indigenenbewegung, die sich seit den 1970er Jahren auf internationaler Ebene etabliert hat, schaffte es, einen grundlegenden Politikwechsel durchzusetzen. Der Umgang mit indigenen Völkern wandelte sich von einer Assimilationspolitik zu einer Beteiligungspolitik, bei der Indigene - zumindest der Form halber - bei internationalen Verhandlungen und Entscheidungsprozessen eingeladen und angehört werden. Die neueren normativen Quellen, insbesondere die bedeutende UNDRIPs von 2007, zeugen von einer Wertschätzung indigener Lebensweise, dem Schutz vor kultureller Assimilation und der Anerkennung eines Selbstbestimmungsrechts. Letzteres bedeutet jedoch nicht, dass indigenen Völkern das Recht auf ,Selbstbestimmung der Völker' im Sinne der UN Charta und damit der Status als Völkerrechtssubjekt zugesprochen wird. Vielmehr fügt sich das ,Selbstbestimmungsrecht indigener Völker' nach jahrelanger Kompromissfindung in den Kontext der internationalen Menschenrechte ein, um dadurch die politische Forderung auf Selbstbestimmung im Sinne von Sezession und die entsprechend aufgeladene Bezeichnung, people ' in ihrer völkerrechtlichen Wirkmächtigkeit einzuschränken und somit für die Staaten - insbesondere die afrikanischen - konsensfähig zu machen.

Die politische Bewegung indigener Völker lässt sich allerdings nicht auf die Menschenrechtsorgane der Vereinten Nationen und das UNPFII reduzieren. Vielmehr spielen indigene Völker in unterschiedlichsten Bereichen auf internationaler Ebene eine Rolle. Die Verschränkungen mit dem internationalen Menschenrechtsschutz, die Präsenz in verschiedenen internationalen Organisationen wie der ILO, der WIPO256 oder im Rahmen der CBD führen dazu, dass die neuen Politiken und normativen Regelungen in unterschiedlichsten Foren und Institutionen artikuliert werden und zur Anwendung kommen. Diese vielschichtige Präsenz indigener Völker als eigenständige Akteure ist das einmalige Ergebnis eines jahrzehntelangen Kampfes von nicht-staatlichen Akteuren um internationale Anerkennung.

\footnotetext{
254 Anaya, Indigenous peoples in international law, S. 259-266.

${ }^{255}$ Clive Baldwin und Cynthia Morel, „Using the United Nations Declaration on the Rights of Indigenous Peoples in Litigation“, in: Steve Allen und Alexandra Xanthaki (Hrsg.), Reflections on the UN Declaration on the Rights of Indigenous Peoples, 2011, S. 121-143.

256 Siehe dazu „Teil 4: Verhandlungen im WIPO Intergovernmental Committee“ auf S. 181.
} 


\section{Lokale Gemeinschaften}

Eng verknüpft mit den Entwicklungen der Rechte indigener Völker entwickelten sich im Kontext des internationalen Umweltrechts eigene Regelungen, unter denen substaatliche Gruppen besondere Rechte genießen. Die Terminologie in diesem Bereich ist jedoch weit gefächert, sie verknüpft und vermischt Gruppenkonzepte mit Organisationen und allgemeinen Begriffen. Die Bandbreite reicht von ,communities', ,populations', ,peoples' über ,community-based organizations', ,international, non-governmental and local community organizations", ,local communities and resource users [...] particularly relevant for rural and urban populations, indigenous, women and youth groups', ,workers', ,farmers', bis hin zu ,local populations', ,local groups ' und dem Begriffspaar der ,indigenous and local community '257 Letzteres findet insbesondere in der Konvention zum Schutz biologischer Vielfalt Verwendung, einem der wichtigsten Instrumente im Umweltvölkerrecht. Insgesamt wird im Kontext der Politiken der Nachhaltigkeit und biologischen Vielfalt der Begriff ,lokale Gemeinschaft ${ }^{\text {‘ }}$ geprägt, der im Folgenden näher dargestellt wird.

I. Geschichte der indigenen und lokalen Gemeinschaften im Umweltvölkerrecht

Die Geschichte substaatlicher Gruppen im Umweltvölkerrecht lässt sich in historisch ältere individuelle Regelungen von ,Kulturausnahmen“258 sowie das moderne Normprogramm für ,lokale Gemeinschaften' und ihr traditionelles Wissen seit den 1990er Jahren unterteilen.

\section{Umweltrechtliche Regelungen zu substaatlichen Gruppen bis 1992}

Im Umweltvölkerrecht findet sich eine Reihe von Ausnahme- und Sonderregelungen für kulturell distinkte, substaatliche Gruppen, die dazu unter verschiedenen, durch den jeweiligen historischen Kontext geprägten Begrifflichkeiten Beachtung fanden. ${ }^{259}$ Die erste solcher Ausnahmeregelungen zugunsten der ,Indianer findet sich bereits 1893 in einem Schiedsspruch zum Schutz und Erhalt pazifischer Pelzrobbenbestände. ${ }^{260}$ In ähnlicher Weise enthält die Convention for the Regulation of Whaling von 1931 eine generelle Ausnahme ihrer Anwendbarkeit für ,aborigines dwelling on the coasts“. ${ }^{261}$ Diese Ausnahme zum allgemeinen

\footnotetext{
${ }^{257}$ Estelle Fach, „Legal Empowerment of Local Communities: a Role for International Environmental Law?", S. 5-8, 14.

258 Kerrin Schillhorn, Kulturelle Rechte indigener Völker und Umweltvölkerrecht - Verbältnis und Vereinbarkeit, 2000, S. 172-185.

259 Ebd., S. 114-123.

${ }^{260}$ Bering Sea Tribunal of Arbitration, „Bering Sea Fur Seals Fisheries Arbitration (Great Britain v. United States)“, Moore's International Arbitration, 1893, S. 755.

261 Convention For The Regulation Of Whaling, 155 LNTS 349, 24. September 1931, Art. 3.
} 
Walfangverbot wurde von der International Whaling Commission unter der International Convention for the Regulation of Whaling von 1946 weiterhin respektiert, führte jedoch im Zuge der aufkommenden Umweltbewegung seit den 1980er Jahren bis heute zu Kontroversen.262 Auch im Übereinkommen zur Erhaltung der wandernden wild lebenden Tierarten von 1979 finden sich Ausnahmeregelungen für ,traditionelle Nutzer'.263

Die Begriffe der ,lokalen Gemeinschaft ${ }^{\natural}$ und der, indigenen Völker finden seit Ende der 1970er, beziehungsweise seit Beginn der 1980er Jahre Verwendung im Umweltvölkerrecht. So erkennt die 1983 von der Generalversammlung eingesetzte World Commission on Environment and Development in ihrem 1987 veröffentlichten Brundtland-Bericht an, dass

local communities [...] so-called indigenous or tribal people [...] are the repositories of vast accumulations of traditional knowledge and experience that links humanity with its ancient origins. [...] It is a terrible irony that as formal development reaches more deeply into rain forests, deserts, and other isolated environments it tends to destroy the only cultures that have proved able to thrive in these environements. ${ }^{264}$

Die Anerkennung dieser substaatlichen Gruppen wird somit in engem Zusammenhang mit dem Gedanken der Anpassungsfähigkeit an extreme Umweltbedingungen und nachhaltiger Entwicklung normiert und insbesondere mit der Nutzung traditionellen Wissens in Verbindung gebracht. ${ }^{265}$ Diese enge inhaltliche Verknüpfung der Gedanken der Abhängigkeit und Nachhaltigkeit von ihrer und für ihre Umwelt lokaler Gruppen wurde 1992 unter dem Begriffspaar der ,indigenous and local communities' auf der United Nations Conference on Environment and Development in Rio de Janeiro (Rio-Konferenø) geprägt. ${ }^{260}$

262 Alexander Gillespie, „Aboriginal Subsistence Whaling: A Critique of the Inter-Relationship Between International Law and the International Whaling Commission", Colorado Jounral of International Environmental Law and Policy, Vol. 12 (2001), S. 77-139; Eine Beschreibung der Regelungen der International Whaling Commission (IWC) zum Aboriginal Subsistence Whaling (ASW) und der für die Anerkannten Gruppen festgelegten Fangquoten findet sich mit weiterführenden links unter: http://iwcoffice.org/conservation/aboriginal.htm.

263 Übereinkommen zur Erhaltung der wandernden wild lebenden Tierarten, 1651 UNTS 333, 23. Juni 1979, Art. II (5) c).

264 UN Generalversammlung, World Commission on Environment and Development, „Our common future“ („Brundtland Report“), UN Doc. A/42/427, 4. August 1987, Part II, Chapter 4, Abs. 70,74 .

265 Anja von Hahn, Traditionelles Wissen indigener und lokaler Gemeinschaften zwischen geistigen Eigentumsrechten und der ,public domain", 2004, S. 83.

266 Einen umfassenden Überblick über die Entwicklung des Umweltvölkerrechts in Hinblick auf indigene und lokale Gemeinschaften findet sich bei: von Hahn, Traditionelles Wissen indigener und lokaler Gemeinschaften zwischen geistigen Eigentumsrecbten und der „public domain“, S. 61-83; Barsh, „Indigenous Peoples“; Darrell A. Posey, „Identifizierung und Respektierung der Grenzen zwischen 


\section{Die Rolle traditionellen Wissens}

Mit ihrem traditionellen Wissen gelten lokale Gemeinschaften als Garant für die nachhaltige Nutzung natürlicher Ressourcen und die Bewahrung hoher biologischer und genetischer Vielfalt genutzter Pflanzen. Eine allgemeine Definition, was von traditionellem Wissen umfasst sein soll, konnte bisher keine allgemeine Anerkennung finden. Ansätze einer Begriffsbestimmung beschreiben traditionelles Wissen als „Wissen über medizinische oder sonstige nützliche Eigenschaften biologischer Vielfalt, das oft seit Generationen in einer bestimmten oder in mehreren Gemeinschaften vorhanden und Teil der kulturellen Identität dieser Gruppen ist.“"267 Traditionelles Wissen wird verstanden als in einer lokalen Gemeinschaft historisch verwurzelt, wo es mündlich von Generation zu Generation tradiert wird. Es beruht auf Erfahrungen, die sich in die lokale Kultur und ihren Umweltkontext einfügen. ${ }^{268}$ Traditionelles Wissen ist dabei kein statischer Bestand an praktischen Kenntnissen zur nachhaltigen Nutzung der gegebenen biologischen Ressourcen, sondern wird verstanden als

cumulative body of knowledge and beliefs, handed down through generations by cultural transmission, about the relationship of living beings (including humans) with one another and their environment. Further, TEK [traditional ecological knowledge] is an attribute of societies with historical continuity in resource use practices; by and large, these are non-industrial or less technologically advanced societies, many of them indigenous or tribal. ${ }^{269}$

Traditionelles Wissen entwickelt sich ständig fort und passt sich so aufbauend in den Kontext des jeweiligen kulturellen Wissensbestandes ein. Die Konvention über die biologische Vielfalt fächert in Artikel 8(j) den Begriff ,traditionelles Wissen' in „Kenntnisse, Innovationen und Gebräuche“ auf. Die Weitergabe traditionellen Wissens kann dabei auch auf einzelne Personen beschränkt sein, die beispielsweise bestimmte Funktionen in einer Gruppe, wie die Ausübung medizinalen Wissens, wahrnehmen.

Die Träger traditionellen Wissens sind indigene und lokale Gemeinschaften, die in Anwendung dieses Wissens eine nachhaltige, Biodiversität bewahrende und

indigenen Völkern, traditionellen Bauern und örtlichen Gemeinschaften“, in: Ernst Ulrich von Weizsäcker (Hrsg.), Grenzen Los?, 1997, S. 242-258.

${ }^{267}$ von Hahn, Traditionelles Wissen indigener und lokaler Gemeinschaften zwischen geistigen Eigentumsrecbten und der ,public domain", S. $7 \mathrm{f}$.

268 Jonathan Curci, The protection of biodiversity and traditional knowledge in international law of intellectual property, 2010, S. 14.

${ }^{269}$ Fikret Berkes, ,'Traditional Ecological Knowledge in Perspective“, in: Julian Inglis (Hrsg.), Traditional Ecological Knowledge: Concepts and Cases, 1993, S. 3. 
fördernde Bewirtschaftung ihres Landes praktizieren.270 So wird argumentiert, dass durch das Zutun lokaler Gemeinschaften die biologische Vielfalt, die zunächst auch ohne vom Menschen gestalteter Einflüsse existiert, durch den Austausch zwischen kultivierten und wilden Arten weiter diversifiziert wurde und beispielsweise durch verschiedenartige Kombinationen von Pflanzen in der Landwirtschaft langfristig erhalten wird. ${ }^{271}$ Gleichzeitig - so stellte es bereits der Brundtland-Bericht fest - befinden sich lokale Gemeinschaften in ihrer traditionellen und nachhaltigen Lebensweise in besonderer Abhängigkeit zu ihrer natürlichen Umwelt. $^{272}$

Diesem Bild lokaler Gemeinschaften und der Bedeutung ihres traditionellen Wissens wird die Kritik entgegengehalten, es sei nur eine naive und romantisierte Idealvorstellung und der traditionellen Subsistenzwirtschaft lokaler Gemeinschaften sei der Gedanke der Nachhaltigkeit nicht von sich aus inhärent. ${ }^{273}$ Vielmehr ergebe sich diese Art der Nutzung durch die geringe Besiedlungsdichte, den geringen Grad an technischer Entwicklung sowie die ausschließliche Nutzung für den Erhalt der Gruppe. Durch äußere Einflüsse, wie etwa die Konzentration lokaler Gemeinschaften auf kleinere Gebiete, die Verbreitung von effektiveren Technologien und eine Verbindung zu Märkten, die einen überregionalen Handel ermöglichen, verändern sich auch die Wirtschaftsformen lokaler Gemeinschaften in einer für die biologische Vielfalt nachteiligen Weise. Die Prämisse des Schutzes traditionellen Wissens lokaler Gemeinschaften zum Schutz biologischer Vielfalt, wie sie auch in der Konvention über die biologische Vielfalt formuliert ist, knüpft allerdings gerade an die traditionelle Wirtschaftsweise an, wie sie diese Gruppen über Generationen hinweg in nachhaltiger Weise entwickelt und dabei biologische Vielfalt bewahrt und verbessert haben.

\section{Der Einfluss der ,Farmer's Rights'}

Die Bedeutung traditionellen Wissens wird insbesondere im Bereich der Landwirtschaft anerkannt. So entwickelte sich seit den 1960er Jahren im Rahmen der Food and Agriculture Organization (FAO) ein Bewusstsein für die Bedeutung von pflanzengenetischen Ressourcen, für welche sogenannte ,Farmer's Rights' zur

\footnotetext{
${ }^{270}$ von Hahn, Traditionelles Wissen indigener und lokaler Gemeinschaften zwischen geistigen Eigentumsrecbten und der, ,public domain", S. 49.

271 Ashish Kothari, Understanding Biodiversity: Life, Sustainability and Equity, 1997, S. 51; Stephen B. Brush und Doreen Stabinsky, V aluing Local Knowledge: Indigenous People and Intellectual Property Rights, 1996, S. 2.

272 UN Generalversammlung, World Commission on Environment and Development, „Our common future“ („Brundtland Report"), UN Doc. A/42/427, Part II, Chapter 4, Abs. 70-78, S. 118-120; von Hahn, Traditionelles Wissen indigener und lokaler Gemeinschaften zwischen geistigen Eigentumsrecbten und der ,public domain", S. 48.

${ }^{273}$ von Hahn, Traditionelles Wissen indigener und lokaler Gemeinschaften zwischen geistigen Eigentumsrecbten und der ,public domain", S. 50.
} 
Bewahrung, Verbesserung und Verfügbarmachung anerkannt sind. ${ }^{274}$ Dabei soll das Konzept der ,Farmer's Rights' nicht nur ,farmer' im wörtlichen Sinne, sondern auch indigene und lokale Gemeinschaften umfassen. ${ }^{275}$ Angestoßen durch entsprechende Aufrufe während der Rio-Konferenz 1992 hat die FAO im International Treaty on Plant Genetic Resources for Food and Agriculture (ITPGRFA) parallel zu den Regelungen in der CBD die

enormous contribution [anerkannt] that the local and indigenous communities and farmers [...] have made and will continute to make for the conservation and development of plant genetic resources which constitute the basis for food and agriculture production throughout the world. ${ }^{276}$

Im ITPGRF $A$ wird auch erklärt, dass der Schutz des traditionellen Wissens mit Bezug zu pflanzengenetischen Ressourcen von den ,Farmer's Rights“ umfasst wird. ${ }^{277}$

\section{Die aktuelle Bedeutung traditionellen Wissens indigener und lokaler Gemeinschaften}

Die Bedeutung von traditionellem Wissen indigener und lokaler Gemeinschaften liegt neben den Belangen im Agrarbereich vor allem im Kontext des medizinalen und pharmakologischen Wissens. So ist es beispielsweise das Wissen über die antibiotischen, steroiden oder Tumorbildung vermindernden Eigenschaften des Erdreichs und von Pflanzen, das für die moderne Chemie- und Pharmaindustrie von Interesse ist. ${ }^{278}$ Auf einer globalen Ebene führte dies zu Konflikten, die unter dem Stichwort Biopiraterie bekannt geworden sind. ${ }^{279}$ Damit wird die kommerzielle Nutzung traditionellen Wissens durch transnationale Chemie- und Biotechnologie-Konzerne bezeichnet, die ohne Einverständnis oder Entschädigung der Gruppen geschieht, von denen dieses Wissen stammt. Insbesondere geht es dabei um die Aneignung dieses Wissens durch Instrumente geistigen Eigentums sowie um die Vermarktung entsprechender Produkte ohne die erziel-

\footnotetext{
${ }^{274}$ Food and Agricultural Organization, „Resolution 5/89, Farmers’ Rights“, Report of the Conference of FAO, 25nd Session, Rom 11.-29. November 1989, V. C 89/REP, 29. November 1989.

275 von Hahn, Traditionelles Wissen indigener und lokaler Gemeinschaften zpischen geistigen Eigentumsrechten und der ,public domain“, S. 80; Peter-Tobias Stoll und Anja von Hahn, „Indigenous peoples, indigenous knowledge and indigenous resources in international law", in: Silke von Lewinski (Hrsg.), Indigenous heritage and intellectual property: genetic resources, traditional knowledge and folklore, 2008, S. $42 \mathrm{f}$.

276 Food and Agricultural Organization, „International Treaty on Plant Genetic Resources for Food and Agriculture“, 2400 UNTS 379, 3. November 2001, Art. 9.

277 Ebd., Art. 9.2, lit. a.

278 von Hahn, Traditionelles Wissen indigener und lokaler Gemeinschaften zwischen geistigen Eigentumsrecbten und der ,public domain", S. 8f, 42-45.

${ }^{279}$ Curci, The protection of biodiversity and traditional knowledge in international law of intellectual property, S. $6 \mathrm{f}$.
} 
ten Gewinne gerecht zu teilen.280 Geopolitisch lassen sich die Konfliktparteien in den biodiversitätsreichen äquatornahen Staaten auf der einen Seite und den westlichen Industriestaaten auf der anderen verorten. Im Kontext dieser Konflikte entwickelte sich im nationalen und internationalen Umweltrecht eine Vielzahl von Instrumenten, die lokale Gemeinschaften mit entsprechenden Rechten zum Schutz und zur Bewahrung ihres traditionellen Wissens ausstatten. Indigene und lokale Gemeinschaften sind im Umweltvölkerrecht also zum einen in Hinblick auf etwaige Ausnahmen, die ihre kulturell gegebene subsistenzwirtschaftliche Lebensweise ermöglichen sollen, zum anderen im Kontext der Konflikte um traditionelles Wissen von Bedeutung.

\section{Rechtsquellen des Konzepts lokaler Gemeinschaften}

Die Regelungen zu lokalen Gemeinschaften im Umweltvölkerrecht sind eng verknüpft mit den Entwicklungen der Rechte indigener Völker. Einerseits konkretisiert das Umweltrecht mit seinen Regelungen zur nachhaltigen Bewirtschaftung von Land in einer funktionellen Überschneidung die Ansprüche auf Selbstbestimmung indigener Völker und dies insbesondere in Bezug auf natürliche Ressourcen. Andererseits spielen indigene Völker mit traditioneller Lebensweise und ihr traditionelles Wissen eine entscheidende Rolle bei der Bewahrung biologischer Vielfalt. Aus einer umweltvölkerrechtlichen Perspektive sind also für eine Untersuchung der Rechte substaatlicher Gruppen die Instrumente und Institutionen zu Rechten indigener Völker mit einzubeziehen. ${ }^{281}$ Allerdings entwickelten sich unter dem Begriff ,lokale Gemeinschaft ${ }^{\star}$ seit der Rio-Konferen ; 1992 eigene, weitergehende Regelungen zu substaatlichen Gruppen im Umweltvölkerrecht, die in ihrer Anwendbarkeit und Regelungsdichte über die Rechte indigener Völker hinausgehen.

Die Rio-Konferenz 1992 reiht sich als Folgeveranstaltung der ersten globalen Umweltkonferenz der Vereinten Nationen 1972 in Stockholm in eine Kette von weiteren umweltvölkerrechtlichen Weltkonferenzen ein. Bisher folgten zwei weitere durch die UN organisierte Veranstaltungen: das World Summit on Sustainable Development 2002 in Johannesburg (WSSD) und die United Nations Conference on Sustainable Development 2012 in Rio de Janeiro (UNCSD), auf denen jeweils weitere Dokumente mit Regelungen zu lokalen Gemeinschaften verabschiedet wurden. Die umweltvölkerrechtlichen Quellen sollen im Folgenden mit einem Fokus auf Regelungen zu lokalen Gemeinschaften skizziert werden.

\footnotetext{
280 Ebd.

281 Dies sind insbsondere die ILO Conventions No. 107 und No. 169, die UNDRIPS sowie das UNPFII. Siehe dazu „B. II. Rechtsquellen und Institutionen“ auf S. 41.
} 


\section{Die Rio-Konferenz 1992}

Auf der Rio-Konferenz wurden 1992 neben der UN Rabmenkonvention zum Klimawandel (UNFCCC) in den Dokumenten der Forest Principles, der Agenda 21, der Rio-Deklaration zu Umwelt und Entwicklung sowie der Biodiversitätskonvention (CBD) Regelungen zu lokalen Gemeinschaften verabschiedet. Insbesondere die Verhandlungen zur $C B D$ entwickelten sich zu einem wichtigen Forum indigener Gemeinschaften, da sie hier in verschiedenen Arbeitsgruppen und Expertentreffen die Möglichkeit der unmittelbaren Einflussnahme hatten. ${ }^{282}$

\section{a) Rio-Deklaration}

Die rechtlich unverbindliche Rio-Deklaration konkretisiert in ihrem 27-Grundsätze-Programm das Verhältnis von Umweltschutz und wirtschaftlicher Entwicklung und erkennt in Grundsatz 22 an, dass

Indigenous people and their communities and other local communities have a vital role in environmental management and development because of their knowledge and traditional practices. States should recognize and duly support their identity, culture and interests and enable their effective participation in the achievement of sustainable development. ${ }^{283}$

Die Rio-Deklaration normiert somit die Verbindung indigener und lokaler Gemeinschaften zu ihrer Umwelt, welche in ihrem Wissen und ihren traditionellen Praxen begründet ist, und erkennt den Zusammenhang ihrer Identität und Kultur mit nachhaltiger Entwicklung an. Indigene und lokale Gemeinschaften haben somit auch in das Grundsatzprogramm des internationalen Umweltrechts Einzug gefunden, in dem ihre Rolle und Verbindung zu nachhaltiger Entwicklung anerkannt werden.

b) Die Biodiversitätskonvention

Die Biodiversitätskonvention normiert den Grundsatz der Bewahrung biologischer Vielfalt, ihre nachhaltige Nutzung sowie den Zugang und die gerechte Vorteilsteilhabe an der Nutzung genetischer Ressourcen. ${ }^{284}$ Sie geht damit über das traditionelle Konzept des umweltrechtlichen Artenschutzes hinaus, indem es welt-

\footnotetext{
282 von Hahn, Traditionelles Wissen indigener und lokaler Gemeinschaften zwischen geistigen Eigentumsrecbten und der ,public domain“, S. 63.

283 UN Generalversammlung, „Rio Declaration on Environment and Development“, UN Doc. A/CONF.151/26(Vol. I), 12. August 1992, Principle 22.

${ }^{284}$ Convention on Biological Diversity, Art. 1.
} 
weit jegliche Ökosysteme, Arten und Gene unter Schutz stellt. ${ }^{285}$ Es enthält Regelungen zur in-situ und ex-situ Erhaltung sowie eine Zugangs- und Teilhabeordnung an genetischen Ressourcen. Als Rahmenkonvention etabliert sie eine Reihe von Organen, welche die Regelungen unter anderem im Rahmen der Vertragsstaatenkonferenz (Conference of the Parties, COP), in ad hoc open ended Working Groups, Kontaktgruppen und in intersessional meetings in Form von zusätzlichen Protokollen ständig weiter konkretisieren. ${ }^{286}$ So spezifizierte das Cartagena Protokoll aus dem Jahr 2000 Fragen zur Biosicherheit und das Nagoya Protokoll von 2010 Einzelheiten zum Zugang und zur Vorteilsteilhabe genetischer Ressourcen (access and benefit sharing).

Für indigene und lokale Gemeinschaften konstatiert die Biodiversitätskonvention in Artikel 8(j), dass

[e]ach Contracting Party shall, as far as possible and as appropriate: $[\ldots]$

(j) Subject to its national legislation, respect, preserve and maintain knowledge, innovations and practices of indigenous and local communities embodying traditional lifestyles relevant for the conservation and sustainable use of biological diversity and promote their wider application with the approval and involvement of the holders of such knowledge, innovations and practices and encourage the equitable sharing of the benefits arising from the utilization of such knowledge, innovations and practices. ${ }^{287}$

Sie erkennt damit nicht nur die Bedeutung indigener und lokaler Gemeinschaften für die Erhaltung einer nachhaltigen Nutzung biologischer Ressourcen an, sondern verpflichtet gleichzeitig die Vertragsstaaten, das traditionelle Wissen dieser Gruppen zu respektieren, zu bewahren und zu erhalten sowie die Anwendung dieses Wissens zu fördern und die Teilung der Vorteile der Nutzung zu fördern.

Weiterhin bestimmt Artikel 10 c) CBD, dass die Vertragsstaaten soweit möglich und angebracht

\footnotetext{
${ }^{285}$ Rüdiger Wolfrum, „Völkerrechtlicher Rahmen für die Erhaltung der Biodiversität“, in: Nina Wolff und Wolfgang Köck (Hrsg.), 10 Jahre Übereinkommen über die biologische Vielfalt: eine Zwischenbilanz, 2004, S. $18 f$.

${ }^{286}$ Ein Überblick findet sich bei Horst Korn, „Institutioneller und instrumenteller Rahmen für die Erhaltung der Biodiversität“, in: Nina Wolff und Wolfgang Köck (Hrsg.), 10 Jabre Übereinkommen über die biologische Vielfalt: eine Zwischenbilan₹, 2004, S. 36-54.

${ }^{287} \mathrm{CBD}$, Art. 8(j).
} 
[p]rotect and encourage customary use of biological ressources in accordance with traditional cultural practices that are compatible with the conservation or sustainable use requirements. 288

Des Weiteren bestimmt Artikel 17 II CBD, dass neben technischer, wissenschaftlicher und sozio-ökonomischer Forschung auch indigenes und traditionelles Wissen Teil des Technologietransfers sein soll.

Die Bestimmungen der $C B D$ begründen für die Vertragsstaaten somit konkrete Handlungspflichten und stellen indigenes Wissen speziell im Kontext der Nutzung genetischer Ressourcen unter völkerrechtlichen Schutz. ${ }^{289}$ Außerdem gibt sie den Rahmen für weitere Verhandlungen und Konkretisierungen vor. So enthält das Cartagena Protokoll zur Biosicherheit beispielsweise explizite Regelungen, welche die Bedeutung von biologischer Vielfalt für indigene und lokale Gemeinschaften bestätigt und zur Kooperation bei Forschung und Informationsaustausch aufruft, um die sozio-ökonomischen Auswirkungen der Handhabung und Verwendung von lebenden modifizierten Organismen besser verstehen und bewältigen zu können. ${ }^{290}$ Im Nagoya Protokoll aus dem Jahr 2010 wurden Regelungen zu traditionellem Wissen, welches an genetische Ressourcen anknüpft, bezüglich Zugang und Vorteilsteilhabe getroffen. Darin werden Bestimmungen über den prior informed consent, die gerechte Vorteilsteilhabe sowie die Berücksichtigung von lokalen Regeln und Verfahren konkretisiert. ${ }^{291}$ Des Weiteren arbeitet gegenwärtig die ad hoc open-ended working group on Article 8(j) an weiteren Details zu diesem Artikel.

Read together, the provision of the CBD secure the rights of indigenous peoples to continue to use living resources in accordance with traditional, sustainable practices; to own, control and perpetuate their traditional ecological knowledge systems and practical know-how with the assistance of the state; and to

\footnotetext{
${ }^{288}$ Ebd., Art. 10 c).

289 Ulrich Beyerlin, ,„Erhaltung und nachhaltige Nutzung“ als Grundkonzept der Biodiversitätskonvention“, in: Nina Wolff und Wolfgang Köck (Hrsg.), 10 Jahre Übereinkommen über die biologische Vielfalt: eine Zwischenbilan₹, 2004, S. 65-68; Peter-Tobias Stoll, „Genetische Ressourcen, Zugang und Vorteilsteilhabe“, in: Nina Wolff und Wolfgang Köck (Hrsg.), 10 Jahre Übereinkommen über die biologische Vielfalt: eine Zwischenbilan₹, 2004, S. 82.

290 Cartagena Protocol on Biosafety to the Convention on Biological Diversity, 2226 UNTS 208, 29. Januar 2000, Art. 26.

291 Conference of the Parties to the Convention on Biological Diversity, „Nagoya Protocol on Access to Genetic Resources and the Fair and Equitable Sharing of Benefits Arising from their Utilization to the Convention on Biological Diversity" (Nagoya Protocol on Access and Benefit-Sharing), UNEP/CBD/COP/DEC/X/1, 29. Oktober 2010.
} 
market their ecological knowledge and know-how if they choose. ${ }^{292}$

Diese Regelungen bilden somit ein konkretes rechtliches Normprogramm mit einer bis dahin noch nicht erreichten Verbindlichkeit, stehen aber gleichzeitig unter der qualitativen Einschränkung, dass nur hergebrachte, traditionelle und nachhaltige Praxen geschützt werden. ${ }^{293}$

c) Agenda 21

Die Agenda 21, das Handlungsprogramm zu Umwelt und Entwicklung der Vereinten Nationen, beschreibt in 40 Kapiteln rechtlich unverbindliche Handlungsgrundlagen, Ziele, Aktivitäten und Möglichkeiten der Umsetzung der wesentlichen Elemente nachhaltiger Entwicklung. ${ }^{294}$ Dabei werden indigene Gemeinschaften explizit in Kapitel 26 neben anderen Akteuren in Section III: Strengthening the Role of Major Groups genannt. ${ }^{295}$ So wird unter anderem Folgendes festgeschrieben:

26.3. In full partnership with indigenous people and their communities, governments and, where appropriate, intergovernmental organizations should aim at fulfilling the following objectives:

(a) Establishment of a process to empower indigenous people and their communities through measures that include: [...]

ii Recognition that the lands of indigenous people and their communities should be protected from activities that are environmentally unsound or that the indigenous people concerned consider to be socially and culturally inappropriate;

iii Recognition of their values, traditional knowledge and resource management practices with a view to promoting environmentally sound and sustainable development. ${ }^{296}$

Dieses Dokument erkennt die Verbindung indigener Gemeinschaften zu ihrem Land und ihre Rolle in der nachhaltigen Bewirtschaftung und Entwicklung an. Außerdem erstrecken sich die gesetzten Ziele auf die Anerkennung der traditio-

\footnotetext{
292 Barsh, „Indigenous Peoples“, S. 848.

293 Ebd.

${ }^{294}$ United Nations Conference on Environment and Developtment, „Report of the United Nations Conference on Environment and Developtment", Annex II, Agenda 21, UN. doc. A/CONF.151/26/Rev.1 (Vol. I), 3.-14. Juni 1992.

${ }^{295}$ Ebd., Section III, Chapter 26. Neben der „Global action for women towards sustainable and equitable development" (Kapitel 24), den Kindern und der Jugend (Kapitel 25), NGOs (Kapitel 27) wird auch die Rolle der Landwirte anerkannt.

${ }^{296}$ Ebd., Section III, Chapter 26.3.
} 
nellen und direkten Abhängigkeit indigener Gruppen von erneuerbaren Ressourcen und Ökosystemen, die als lebenswichtig für ihr kulturelles, wirtschaftliches und physisches Wohlergehen angesehen werden. Schließlich wird als Handlungsempfehlung ein Mehr an Einflussnahme indigener Völker über ihr Land, die Selbstorganisation ihrer Ressourcen sowie Teilhabe an sie betreffenden Entscheidungsprozessen angemahnt. ${ }^{297}$ Die Bedeutung traditionellen Wissens wird mehrfach betont und Kapitel 15 zur Regelung des Schutzes biologischer Vielfalt normiert in Absatz 4 und 5, dass

15.4. Governments at the appropriate level, [...] taking into consideration indigenous people and their communities, as well as social and economic factors, should: [...]

(g) Recognize and foster the traditional methods and the knowledge of indigenous people and their communities, emphasizing the particular role of women, relevant to the conservation of biological diversity and the sustainable use of biological resources, and ensure the opportunity for the participation of those groups in the economic and commercial benefits derived from the use of such traditional methods and knowledge; [...]

15.5 (e) Subject to national legislation, take action to respect, record, protect and promote the wider application of the knowledge, innovations and practices of indigenous and local communities embodying traditional lifestyles for the conservation of biological diversity and the sustainable use of biological resources, with a view to the fair and equitable sharing of the benefits arising, and promote mechanisms to involve those communities, including women, in the conservation and management of ecosystems; 298

Insgesamt werden also die Belange indigener Gemeinschaften und die Bedeutung ihres traditionellen Wissens im Zusammenhang mit Umweltschutz und Entwicklung mehrfach in unterschiedlichen Kapiteln der Agenda 21 genannt. ${ }^{299}$ Das Handlungsprogramm der internationalen Staatengemeinschaft weist indigenen Völkern somit eine entscheidende Rolle zu.

\footnotetext{
${ }^{297}$ Ebd., Section III, Chapter 26.4.

${ }^{298}$ Ebd., Section II, Chapter 15.4 und 15.5; der Wortlaut von Absatz 5 wurde dem gleichzeitig verhandelten Übereinkommen über die biologische Vielfalt entnommen.

${ }^{299}$ M.w.N. von Hahn, Traditionelles Wissen indigener und lokaler Gemeinschaften zwischen geistigen Eigentumsrechten und der ,public domain", S. 68-71.
} 
d) Walderklärung

Auch in der rechtlich ebenfalls unverbindlichen Walderklärung (Forest Principles) werden die Regierungen aufgefordert, indigene Gruppen und lokale Gemeinschaften bei der Entwicklung, Planung und Umsetzung nationaler Waldpolitiken zu beteiligen und darin die Identität, Kultur und Rechte indigener Völker, ihrer Gemeinschaften und anderen Gemeinschaften von Waldbewohnern anzuerkennen und zu unterstützen. ${ }^{300}$ Dahingehend sollen Regelungen für den Landbesitz für betroffene Gruppen gefunden werden, die als Anreiz für eine nachhaltige Bewirtschaftung der Wälder dienen, sowie die Problematik von Armut und Mangel an Alternativen für lokale Gemeinschaften angegangen werden, die zumeist einer nachhaltigen Bewirtschaftung entgegenstehen. ${ }^{301}$ Schließlich wird auch proklamiert, dass

[a]ppropriate indigenous capacity and local knowledge regarding the conservation and sustainable development of forests should, through institutional and financial support and in collaboration with the people in the local communities concerned, be recognized, respected, recorded, developed and, as appropriate, introduced in the implementation of programmes. ${ }^{302}$

Auch die Walderklärung erkennt somit die Bedeutung indigener Kapazitäten und lokalen Wissens für die nachhaltige Entwicklung von Wäldern an.

\section{2. Übereinkommen zur Bekämpfung der Wüstenbildung}

Auch im Übereinkommen zur Bekämpfung der Wüstenbildung von 1994 finden sich Regelungen zu ,lokalen Gemeinschaften', da es langfristiger Strategien zur Verbesserung der Lebensumstände insbesondere auf lokaler Ebene bedarf, um Wüstenbildung zu verhindern. ${ }^{303}$ Die Konvention normiert dabei Zusammenhänge zwischen Wüstenbildung und ihren physikalischen, biologischen und sozio-ökonomischen Ursachen und ruft zu ihrer Bekämpfung auch zur Einbe-

\footnotetext{
300 United Nations Conference on Environment and Developtment, „, Report of the United Nations Conference on Environment and Developtment", Annex III, Non-legally binding Authoritative Statement of Principles for a Global Consensus on the Management, Conservation and Sustainable Development of all Types of Forests (Forest Principles), UN Doc. A/CONF.151/26(Vol. III), 14. August 1992, Principles 2 (d) und 5 (a).

301 Ebd., Prinzipien 5 (a) und 9 (b).

302 Ebd., Prinzip 12 (d).

303 UN Generalversammlung, Intergovernmental Negotiating Committee for the Elaboration of an International Convention to Combat Desertification in those Countries Experiencing Serious Drought and/or Desertification, Particularly in Africa, „United Nations Convention to Combat Desertification in those Countries Experiencing Serious Drouhgt and/or Desertification, Particularly in Africa" (Convention to Combat Desertification), UN Doc. A/AC.241/27, 12. September 1994, Art. 2.
} 
ziehung von Strategien zur Armutsbekämpfung auf.304 Vergleichbar dem Verständnis der Walderklärung impliziert das Übereinkommen somit, dass lokale Gemeinschaften in ihrer Armut unter Umständen zur Sicherung ihres Überlebens nicht notwendigerweise nachhaltig wirtschaften. ${ }^{305}$ Gleichzeitig erkennt das Übereinkommen die Bedeutung von lokalem und traditionellem Wissen an, ruft zum Austausch dieses Wissens und zur gerechten Teilung der Vorteile dieses Wissens auf. 306

\section{World Summit on Sustainable Development}

Das World Summit on Sustainable Development von 2002 in Johannesburg war die Folgeveranstaltung zur Rio-Konferenz, von 1992. Hier wurden die beiden Kerndokumente The Johannesburg Declaration on Sustainable Development: From our Origins to our Future, welche die wesentliche Rolle indigener Völker in der nachhaltigen Entwicklung bekräftigt, und der Plan of Implementation of the World Summit on Sustainable Development verabschiedet. ${ }^{307}$ Letzteres enthält eine Reihe von Regelungen zu indigenen und lokalen Gemeinschaften, die sich im Grunde auf die erklärten Ziele von Rio beziehen.

So wird darin die Notwendigkeit von Politiken genannt, welche die Abhängigkeit indigener Gemeinschaften von ihrer Umwelt anerkennen und Landrechte für indigene Gemeinschaften fördern, damit sie ihr Land im Sinne eines GemeingutNutzungssystem bewirtschaften können. ${ }^{308}$ Die Bedeutung indigener und lokaler Gemeinschaften und ihres traditionellem Wissens werden weiterhin in zahlreichen Kontexten anerkannt: bei der Energieversorgung und der nachhaltigen Landnutzung, im Ökotourismus, beim nachhaltigen Schutz und der Bewahrung von Biodiversität oder auch in Bezug auf medizinales Wissen. ${ }^{309}$ Insbesondere Artikel 42

\footnotetext{
304 Ebd., Art. 4 II (c).

305 von Hahn, Traditionelles Wissen indigener und lokaler Gemeinschaften zwischen geistigen Eigentumsrechten und der ,public domain", S. 73.

306 Convention to Combat Desertification, Art. $16 \mathrm{ff}$.

307 World Summit on Sustainable Development, ,'The Johannesburg Declaration on Sustainable Development: From Our Origins to the Future“, UN Doc. A/Conf.199/20, 4. September 2002, Abs. 25.

308 World Summit on Sustainable Development, „Plan of Implementation of the World Summit on Sustainable Development“, UN Doc. A/Conf.199/20, 4. September 2002, Art. 6 (e) und (h).

${ }^{309} \mathrm{Im}$ Einzelnen finden sich im „Plan of Implementation of the World Summit on Sustainable Development" Bezüge zu indigenen und lokalen Gemeinschaften bei folgenden Themen: Energieversorgung (Art. 19 (g)); traditionelles und indigenes Wissen im Umgang mit Naturkatastrophen (Art. 25 (f)); Klimawandelfolgenabschätzung (Art. 36 (i)); nachhaltige Landnutzung und Landwirtschaft durch indigene und lokale Ansätze (Art. 38 (d) und (r)); Gebirgsökosystemmanagement (Art. 40 (e)); Ökotourismus (Art. 40 (b) und Art. 64 (c)); Nachhaltiger Schutz und Bewahrung von Biodiversität (Art. 42 (h) und (k)); Teilhabe and Entscheidungen und zur Nutzung von traditionellem Wissen (Art. 42 (l)); Waldwirtschaft im Sinne von Wirtschaftssystemen von indigenen und lokalen Gemeinschaften (Art. 43 (h)); im Bergbau (Art. 44 (b)); bei Nutzung
} 
zur Biodiversität bekräftigt die Rolle indigener und lokaler Gemeinschaften und ruft dazu auf

to $[. .$.$] recognize the rights of local and indigenous communities$ who are holders of traditional knowledge, innovations and practices, and, with the approval and involvement of the holders of such knowledge, innovations and practices, develop and implement benefit-sharing mechanisms on mutually agreed terms for the use of such knowledge, innovations and practices. ${ }^{310}$

Im Wortlaut bezugnehmend auf die Biodiversitätskonvention wird also auch hier die Bedeutung traditionellen Wissens betont und ein Mechanismus zum Vorteilsausgleich für die Nutzung dieses Wissens, der Innovationen und Praxen gefordert, der auf einvernehmlich vereinbarten Bedingungen beruhen soll.

\section{UN Conference on Sustainable Development}

Im Juni 2012 wurde Rio+20, die nächste Folgekonferenz in der Reihe der Weltumweltkonferenzen der Vereinten Nationen, unter dem Titel United Nations Conference on Sustainable Development abgehalten. An der Veranstaltung nahmen Vertreter von über 190 Staaten sowie über 50.000 Vertreter der Zivilgesellschaft an sogenannten side events teil. Das Outcome Document - The Future we want fasst den Konsens der Staatengemeinschaft zu den beiden Hauptthemen der Konferenz, green economy und Verbesserung der internationalen Kooperation, zusammen. Es behandelt den Kampf gegen Armut, die Anerkennung und Bestätigung der Rio Richtlinien und bereits bestehender Umwelt- und Nachhaltigkeitsstrategien, fordert die Entwicklung einer Wirtschaft basierend auf nachhaltiger Entwicklung und der Armutsbekämpfung, bekräftigt den institutionellen Rahmen der nachhaltigen Entwicklung, also die Einbindung des Leitbilds in die politischen Systeme der UN-Mitgliedstaaten und auf internationaler Ebene und beschließt die Erhebung des UN-Umweltprogramms (UNEP) zu einer vollwertigen UN-Agentur.

Ohne neue verbindliche Regelungen zu schaffen, setzt sich die Bekräftigung der Bedeutung lokaler Gemeinschaften für sowie ihre Abhängigkeit von ihrer Umwelt fort.

We recognize that the traditional knowledge, innovations and practices of indigenous peoples and local communities make an important contribution to the conservation and sustainable use of biodiversity, and their wider application can support social

und Verbreitung von traditioneller Medizin (Art. 47 (h) und Art. 58 (d)); Bekämpfung von Wüstenbildung (Art. 57).

310 Ebd., Art. 42 (j). 
well-being and sustainable livelihoods. We further recognize that indigenous peoples and local communities are often the most directly dependent on biodiversity and ecosystems and thus are often the most immediately affected by their loss and degradation. 311

Des Weiteren wird betont, dass ihre Einbeziehung in juristische und administrative Verfahren wichtig für nachhaltige Entwicklung sei. Es wird die Notwendigkeit gleichberechtigten Zugangs zu Bildung und die Bedeutung indigener Völker und lokaler Gemeinschaften für die Nahrungssicherheit in ländlichen Gebieten, die Notwendigkeit des Zugangs zu Fischgründen sowie ihre Bedeutung in Bergregionen anerkannt. Zudem wird betont, dass Politiken der green-economy dem Wohl indigener Völker, lokaler und traditioneller Gemeinschaften und von Minderheiten dienen solle. Weiterhin wird zum Ausbau von Öko-Tourismus und zur finanziellen Unterstützung von indigenen Völkern und lokalen Gemeinschaften zur Gründung kleiner und mittlerer Unternehmen in diesem Bereich aufgerufen. Insgesamt wird also die Einbeziehung indigener Völker und lokaler Gemeinschaften im umweltvölkerrechtlichen Normprogramm in unterschiedlichsten Ausformungen fortgesetzt.

Hervorzuheben ist die begriffliche Schärfung, dass, indigene Völker ${ }^{6}$ nicht mehr wie in den meisten Dokumenten von 1992 und 2002 im Begriffspaar, indigene und lokale Gemeinschaft ${ }^{`}$ aufgehen. Vertreter indigener Völker konnten zumindest mit der Verwendung des Begriffs, indigene Völker ${ }^{6}$ neben dem der ,lokalen Gemeinschaft' sowie mit der Anerkennung der Bedeutung der UNDRIPs von 2007 für die Umsetzung nachhaltiger Entwicklungsstrategien ihre Anliegen im Abschlussdokument durchsetzen. ${ }^{312}$

\section{Verhandlungen zu Rechten geistigen Eigentums und genetischen Ressourcen}

Die Rechte lokaler Gemeinschaften sind auch Gegenstand der gegenwärtigen Verhandlungen im Intergovernmental Committee der World Intellectual Property Organization (WIPO IGC) zu Rechten geistigen Eigentums und genetischer Ressourcen, traditionellem Wissen und traditionellen kulturellen Ausdrucksformen (GR/TK/TCEs). ${ }^{313}$ Von Bedeutung im umweltrechtlichen Kontext sind dabei insbesondere die Verhandlungen zum Themenbereich genetischer Ressourcen und assoziiertem traditionellen Wissen. Die Agenda des IGC zum Themenkom-

311 UN Generalversammlung, „The future we want“, UN Doc. A/Res/66/288, 11. September 2012, Abs. 197.

312 Ebd., Abs. 49; Tebtebba, „Final Statement and Question from Indigenous Peoples Major Group“, 11. April 2012.

313 Eine ausführliche Darstellung der Geschichte und Hintergründe des WIPO IGC findet sich unter „A. Geschichte und Hintergrund“ auf S. 182. 
plex genetische Ressourcen strukturierte sich von Beginn an um die drei Fragen der

(a) defensive protection of genetic resources;

(b) disclosure requirements in patent applications for information related to genetic resources used in the claimed invention; and

(c) IP issues in mutually agreed terms for the fair and equitable sharing of benefits arising from the use of genetic resources. ${ }^{314}$

Dabei zeigten die Verhandlungen der letzen zehn Jahre im IGC zu den Fragen genetischer Ressourcen und geistigen Eigentums einen weniger kompromissfähigen Verlauf als bei den anderen beiden verhandelten Themenbereichen TK und TCEs. ${ }^{315}$ Zwar engagierten sich die westlichen Staaten hier mehr mit eigenen Beiträgen und Vorlagen, machten dabei aber gleichzeitig ihre Position und mangelnde Kompromissbereitschaft gegenüber den Forderungen der lateinamerikanischen, afrikanischen und asiatischen Staatengruppen deutlich. So wurde im Oktober $2011 \mathrm{im}$ Dokument zum zukünftigen Arbeitsprogramm der Themencluster (c) zur Gestaltung einvernehmlicher Vertragsklauseln zur gerechten Vorteilsteilhabe gestrichen, da eine Einigung in diesem Bereich nicht möglich zu sein schien. ${ }^{316}$

Die Verhandlungen im IGC befassen sich somit gegenwärtig nur noch mit zwei Fragen zu genetischen Ressourcen: Zum einen ist die Entwicklung von Datenbanken und Informationssystemen zur defensive protection bei Patentanmeldungen Verhandlungsgegenstand. Eine Einigung hier würde im Ergebnis keine grundlegenden Neuerungen im gegenwärtigen System des internationalen Patentrechts bedeuten und nur bestehende Verfahren zur Beachtung von prior art, dem bereits öffentlichen Wissen, verbessern. Zum anderen werden Regelungen zur zwingenden Angabe der Herkunft genetischer Ressourcen in Patentanmeldungen diskutiert. Dies würde Unternehmen in der Pharmaindustrie erhebliche finanzielle und moralische Kosten aufbürden. Zwar kommt solch eine Offenlegung der Herkunft genetischer Ressourcen keiner unmittelbaren Beteiligung der lokalen Gemeinschaften des Herkunftsortes gleich, ermöglicht aber ein Nachvollziehen der Forschungsgeschichte. Dies wiederum liefert die notwendigen Informationen, die als Grundlage moralischer Ansprüche der Vorteilsteilhabe dienen könnten.

Die Verhandlungen im WIPO IGC zu GR/TK/TCEs entwickeln für den Themenbereich genetischer Ressourcen somit neue Regelungen zu Rechten geistigen Eigentums, die im umweltrechtlichen Kontext für lokale Gemeinschaften von

\footnotetext{
314 WIPO IGC, „Overview of the Committee's Work on Genetic Resources“, WIPO/GRTKF/IC/8/9, 20. April 2005, Abs. 2.

315 Siehe dazu „A. III. Verlauf der Verhandlungen des Intergovernmental Committee“ auf S. 189.

316 WIPO IGC, „Options for Future Work on Intellectual Property and Genetic Resources“, WIPO/GRTKF/IC/20/5, 10. Oktober 2011, Abs. 6 (b).
} 
Bedeutung sind. In Anbetracht des von den westlichen Staaten verzögerten Verlaufs der Verhandlungen und der zunehmenden Einschränkung der diskutierten Regelungsansätze sind die potentiellen Ergebnisse der IGC Verhandlungen jedoch weiterhin offen.

\section{Regionale Instrumente}

Auch auf regionaler Ebene finden sich internationale Regelungen zu lokalen Gemeinschaften, die das Normprogramm der CBD weiterführen und konkretisieren. So entwickelte beispielsweise die Afrikanische Union die Model Legislation for the Recognition and Protection of the Rights of Local Communities, Farmers and Breeders, and for the Regulation of Access to Biological Resources, welches Regelungen zum prior informed consent der lokalen Gemeinschaft über den Zugang zu genetischen Ressourcen trifft sowie ihre Vorteilsteilhabe normiert. ${ }^{317}$ Die Andean Community verabschiedete 1996 ein Common Regime on Access to Genetic Resources, in der die Mitgliedstaaten

recognize and value the rights and the authority of the native, Afro-American and local communities to decide about their know-how, innovations and traditional practices associated with genetic resources and their by-products. ${ }^{318}$

Weiterhin werden Regelungen getroffen, dass die Verträge zum Zugang zu genetischen Ressourcen eine faire und gerechte Vorteilshabe enthalten müssen. ${ }^{319}$ Auch die Association of Southeast Asian Nations hat mit dem Entwurf eines ASEAN Framework. Agreement on Access to Biological and Genetic Resources Regelungen zur Beteiligung indigener Völker und lokaler Gemeinschaften in Form eines prior informed consent und zur fairen und gerechten Vorteilsteilhabe getroffen. ${ }^{320}$ Die geopolitische Konstellation der Konflikte zwischen biodiversitätsreichen äquatornahen Staaten auf der einen Seite und westlichen Industrienationen auf der Anderen macht ein globales System zum Zugang und zur Vorteilsteilhabe der

\footnotetext{
317 Organisation of the African Unity, „Model Legislation for the Recognition and Protection of the Rights of Local Communities, Farmers and Breeders, and for the Regulation of Access to Biological Resources", gebilligt im Rahmen der 74. Sitzung des Ministerrates der OAU vom 5.-8. Juli 2001, CM/Dec.44 (LXXIV), Part II, Art. 1, Stichwort „local community“; Part III, Art. 5; Part IV, Art. 16 und 18; Stoll und von Hahn, „Indigenous peoples, indigenous knowledge and indigenous resources in international law", S. 46f.

318 The Commission of the Cartagena Agreement („Andean Community“), „Decision 391 - Common Regime on Access to Genetic Resources", Official Gazette of the Cartagena Agreement No. 213, 2. Juli 1996, Art. 7.

319 Ebd., Art. 35; Stoll und von Hahn, „Indigenous peoples, indigenous knowledge and indigenous resources in international law", S. 45f.

320 Association of Southeast Asian Nations, „ASEAN Framework Agreement on Access to Biological and Genetic Resources (Draft Text)“, 24. Februar 2000, Art. 10 und 11.
} 
Nutzung genetischer Ressourcen allerdings unumgänglich. Die regionalen Regelungen können somit nur Ansätze bleiben, um einen Ausgleich zwischen lokalen Gemeinschaften auf der einen Seite und den Nutzern im, Westen' zu regulieren.

\section{Regelungen zum Schutz traditionellen Wissens}

Die umweltvölkerrechtlichen Regelungen zu Rechten lokaler Gemeinschaften entwickelten in zunehmender Konkretisierung ein Normprogramm, welches kollektive Rechte an traditionellem Wissen, insbesondere solchem in Bezug zu genetischen Ressourcen, sowie Beteiligungsrechte in hinreichend klar umrissene Formen führt. Zwar findet sich noch kein internationales Instrument, welches diesen Gruppen ein international einklagbares Recht einräumt. Die Zielrichtung und Struktur eines Regelungsrahmens zeichnet sich aber insbesondere durch das Nagoya Protokoll ab.

\section{Traditionelles Wissen und access and benefit sharing}

Die normative Bedeutung lokaler Gemeinschaften liegt hauptsächlich in ihrem traditionellen Wissen zur nachhaltigen Landnutzung und einer Biodiversität fördernden Lebensweise. ${ }^{321}$ Der Kern zur Anknüpfung von Rechten liegt somit im traditionellen Wissen, welches insbesondere im Bezug zu Kenntnissen über Pflanzen und somit pflanzengenetischen Ressourcen für die Agrarwirtschaft sowie für die pharmakologische und medizinische Forschung von Interesse ist. Traditionelles Wissen ist dabei kein statischer Bestand an Wissen, sondern entwickelt sich zum einen im Kontext seiner Träger in Reaktion zu Umwelteinflüssen weiter, zum anderen wird es über Generationen hinweg überliefert, dabei ständig angepasst und weiter entwickelt.

Trotz dieses innovativen Elements traditionellen Wissens genießt es nicht den Schutz der bestehenden Instrumente geistigen Eigentums. Dies liegt einerseits an der meist nur mündlichen Übermittlung und somit mangelnder Manifestation des Wissens. Eine solche ist jedoch Voraussetzung für die Anwendbarkeit von Urheberrechten. Andererseits mangelt es oft an Wissen und den notwendigen finanziellen Mitteln um Patentrechte anzumelden und juristisch zu verteidigen. Teilweise ist es auch gar nicht im Interesse der Trägergruppen ihr traditionelles Wissen im Rahmen der westlichen Instrumente geistigen Eigentums schützen zu lassen. Daraus ergibt sich das bereits dargestellte Spannungsverhältnis: Obwohl traditionelles Wissen über Generationen hinweg entwickelt und bewahrt wurde, können Dritte, also zumeist private oder öffentlich-rechtliche Forschungsinstitutionen, im Zuge der Kommerzialisierung von Innovationen durch Instrumente geistigen Eigentums nur durch einen geringen eigenen Beitrag zur Entwicklung der Marktreife erhebliche ökonomische Vorteile erzielen. Gegenstand dieses Problemfeldes ist

321 Barsh, „Indigenous Peoples“, S. 840. 
insbesondere traditionelles Wissen in Bezug auf pflanzengenetische Ressourcen, für welches sich mit der CBD, den Bonn Guidelines und dem Nagoya Protokoll zunehmend konkrete Rahmenbedingungen für ein System des access and benefit sharing $(A B S)$ entwickelt haben.

Die rechtlichen Rahmenbedingungen des $A B S$ basieren auf der normativen Aussage des Artikels $15 \mathrm{CBD}$, welcher die Souveränität der Staaten über seine natürlichen Ressourcen anerkennt und ihm die ausschließliche Befugnis einräumt, den Zugang zu genetischen Ressourcen bestimmen zu können. ${ }^{322}$ Das Nagoya Protokoll konkretisiert, wie der rechtliche Rahmen auf nationaler Ebene ausgestaltet werden soll, um einerseits den Zugang zu genetischen Ressourcen und dem zugehörigen traditionellen Wissen unter Beachtung einer informierten Zustimmung (prior informed consent) der beteiligten Akteure, also insbesondere indigener und lokaler Gemeinschaften, zu garantieren. Andererseits soll sichergestellt werden, dass die Vorteile der Nutzung und einer Kommerzialisierung fair und gerecht geteilt werden (fair and equitable benefit sharing). Für die beteiligten Gruppen geht es also um das Recht, nur durch informierte Zustimmung den Zugang für Forschung an ,ihren' pflanzengenetischen Ressourcen und ihrem zugehörigen traditionellen Wissen zu erlauben sowie schließlich an etwaigen Gewinnen beteiligt zu werden.

\section{Staatliche Souveränität über genetische Ressourcen}

Indigene und lokale Gemeinschaft stehen als eigentliche Träger traditionellen Wissens ihren Heimatstaaten, denen die Souveränität über die genetischen Ressourcen ihres Landes in der CBD normativ zugeordnet wurde, sowie weltweiten Forschungseinrichtungen als potentielle Nutzer gegenüber. Die Regierungen ihrer Heimatstaaten müssen in der Umsetzung internationaler Regelungen, insbesondere des Nagoya Protokolls, die Rolle der Gruppen im Zustimmungsverfahren berücksichtigen. Dies impliziert, dass die Regierungen die Beteiligung lokaler Gemeinschaften so auszugestalten haben, dass eine informierte Zustimmung oder auch Ablehnung durch die Gruppe zur zwingenden Voraussetzung für Forschungsvorhaben in den Gebieten wird, in denen sie leben. Nur wenn die Rolle der Gruppen in den nationalen Gesetzen verankert ist, haben die Gruppen die notwendige, in den internationalen Instrumenten angedachte Entscheidungsbefähigung, sich gegenüber den Forschungseinrichtungen durchzusetzen.

Des Weiteren ergibt sich ein Verhältnis zwischen lokalen Gemeinschaften auf der einen und Forschungseinrichtungen auf der anderen Seite. Dabei werden letztere den Gruppen in ökonomischer Hinsicht und in rechtlicher Kompetenz im Umgang mit den einschlägigen Regelungen des $A B S$-Systems und den Instrumenten geistigen Eigentums meist weit überlegen sein. So sind es Universitäten, multinationale Pharmakonzerne und Firmen medizinischer Produkte, die im Hinblick

\footnotetext{
322 Nagoya Protocol on Access and Benefit-Sharing, Art. 15.
} 
auf Grundlagenforschung oder in kommerzieller Hinsicht ein Interesse an den genetischen Ressourcen und dem traditionellen Wissen indigener und lokaler Gemeinschaften haben.

IV. Nationalgesetzlicher Rahmen und Rechte geistigen Eigentums

Die rechtlichen Durchsetzungsmechanismen der Rechte indigener und lokaler Gemeinschaften werden ausschließlich auf nationaler Ebene ausgestaltet. Auf internationaler Ebene finden sich keine Institutionen, bei denen umweltrechtliche Ansprüche geltend gemacht werden können. Dies gilt sowohl für Rechte aus dem Umweltvölkerrecht als auch für vergleichbare Instrumente geistigen Eigentums.

\section{Rechtsdurchsetzungsregelungen im Nagoya Protokoll}

Ausgehend von den Vorgaben zur Ausgestaltung der nationalgesetzlichen Rahmenbedingungen im Nagoya Protokoll ist für die konkrete gesetzliche Umsetzung der Regelungen der informierten Zustimmung betroffener Gruppen der politische Wille auf nationaler Ebene entscheidend. ${ }^{323}$ Aus den nationalen Gesetzen ergibt sich der Gestaltungsspielraum für die individuellen $A B S$-Verträge zwischen Forschungsinstitutionen auf der einen sowie den beteiligten Gruppen und ihren Heimatstaaten auf der anderen Seite. ${ }^{324}$ Des Weiteren sollen für die Durchsetzung der Ansprüche an der gerechten Vorteilsteilhabe die anwendbaren Strukturen für Streitschlichtungsverfahren in jedem $A B S$-Vertrag individuell geregelt werden. ${ }^{325}$ Trotz der im Nagoya Protokoll konkretisierten Rahmenbedingungen unterliegen somit sowohl die Vertragsgestaltung als auch die Durchsetzung im Streitfall dem soeben dargestellten Machtungleichgewicht der beteiligten Akteure. ${ }^{326}$ Für eine lokale Gemeinschaft können etwaige Voraussetzungen einer zivilrechtlichen Klage im eigenen Land oder gar im Staat der Forschungseinrichtung eine faktisch unüberwindbare Hürde darstellen.

\section{Schutz durch Rechte geistigen Eigentums}

Die Entwicklungen der letzten Jahre zeigen, dass vereinzelt betroffene Gruppen - meist durch Unterstützung internationaler NGOs - versuchen, sich im Rahmen der bestehenden Rechtsinstrumente geistigen Eigentums gegen die Problematik der Biopiraterie zur Wehr zu setzen. Dabei beschränken sich die An-

\footnotetext{
323 Ebd., Art. 6f.

324 Ebd., Art. 5-7.

325 Ebd., Art. 15-18.

326 Eine umfassende Analyse der vertraglichen Sicherung der Interessen indigener und lokaler Gemeinschaften findet sich bei: von Hahn, Traditionelles Wissen indigener und lokaler Gemeinschaften zimischen geistigen Eigentumsrechten und der „public domain“, S. 206-268.
} 
spruchsgrundlagen lokaler Gemeinschaften im Kontext der übergeordneten Problematik auf die Anfechtung gewährter Patente mangels Neuheit oder mangels unzulässiger Patentierbarkeit. ${ }^{327}$ Trotz des internationalen Rahmens des TRIPS-Abkommens ist das Widerspruchsverfahren mangels Neuheit oder mangels Patentierbarkeit, bei dem die technisch hoch komplexen Biopatente auf individueller Basis in ihrer naturwissenschaftlichen Aussage angegriffen werden müssen, in die jeweiligen nationalen Rechtsordnungen zersplittert, sehr teuer und langwierig. ${ }^{328}$ Die Entwicklungen in der CBD sind dahingehend bereits eine Reaktion auf die Probleme im bestehenden System der Rechte geistigen Eigentums.

\section{Zusammenfassung: neue Akteure beim Schutz von Biodiversität}

Historisch finden sich im internationalen Umweltrecht einige Sonderregelungen für kulturelle Praxen der ,Indianer', tribal people oder ,Aborigines‘. Seit den 1990er Jahren hat sich um den Begriff der ,lokalen Gemeinschaften“ eine Reihe von unterschiedlichen umweltvölkerrechtlichen Instrumenten entwickelt, die Gruppen auf lokaler Ebene in ihr Normprogramm einbeziehen. Insbesondere im Rahmen der $C B D$ hat sich die Staatengemeinschaft für, indigene und lokale Gemeinschaften' auf international verbindliche Regeln geeinigt, welche die Bedeutung genetischer Ressourcen und assoziierten traditionellen Wissens substaatlicher Gruppen für die nachhaltige Bewahrung und Förderung biologischer Vielfalt sowie ihre gleichzeitige Abhängigkeit von ihrer Umwelt normativ anerkennt und einen völkerrechtlichen Regelungsrahmen zum access and benefit sharing garantiert. Es ergibt sich somit im Interesse des internationalen Umweltschutzes eine Verbindung von Entwicklungen der Rechte indigener Völker und der Farmer's Rights, welche die kulturellen Praxen substaatlicher Gruppen in ihrer ökologischen Nachhaltigkeit aufgreift und eigene politische Bewegungen und Prozesse internationaler Normsetzung mit globaler Anwendbarkeit für lokale Gemeinschaften etabliert.

\footnotetext{
$327 \mathrm{Zu}$ den Rechten Dritter in Konflikten um traditionelles Wissen und traditionelle Pflanzensorten siehe: Ebd., S. 269-310.

328 Ein Überblick über die Problematik im europäischen System findet sich in: Malwina Mejer und Bruno van Pottelsberghe de la Potterie, „Economic Incongruities in the European Patent System“, European Journal of Law and Economics, Vol. 34, Nr. 1 (2011), S. 215; eine Aufstellung der Kosten findet sich auf S. 219.
} 



\section{Teil 2: Definitionen}

Die völkerrechtlichen Instrumente, die Interessen und Rechte substaatlicher Gruppen normieren, weisen trotz ihrer unterschiedlichen Entstehungsgeschichten erhebliche Überschneidungen bei der Frage auf, wie kulturell distinkte Gruppen rechtlich zu definieren sind. Für alle Konzepte substaatlicher Gruppen wurde und wird in Verhandlungen internationaler Organisationen, bei internationalen Expertenkommissionen und in der Wissenschaft nach rechtlichen Definitionen gesucht, die abstrakt-generell jeweils die Eigenschaften und Differenzierungsmerkmale von Minderheiten, indigenen Völkern oder lokalen Gemeinschaften beschreiben sollen. So entstand eine Vielzahl sogenannter, Arbeitsdefinitionen', die jeweils eine erste Annäherung an die jeweiligen Begrifflichkeiten darstellen. Jedoch konnte sich bisher keiner der Ansätze einer Definition substaatlicher Gruppen durchsetzen. Die bisherigen Ansätze lassen sich über Verhandlungsprotokolle, Arbeiten von Sonderberichterstattern und wissenschaftliche Sekundärliteratur erschließen. Diesen Quellen folgend sollen im Folgenden die hinter den völkerrechtlichen Begriffen liegenden Konzepte von Minderheiten, indigenen Völkern sowie lokalen Gemeinschaften nachgezeichnet und verglichen werden. 


\section{A. Minderheiten}

In der Geschichte des internationalen Minderheitenschutzes findet sich eine Reihe von Ansätzen einer allgemein anwendbaren Definition des Minderheitenbegriffs. Insbesondere bei der Erarbeitung neuer völkerrechtlicher Instrumente stellte sich immer wieder die Frage nach einer Definition, die eine rechtstechnische Anwendbarkeit gewährleisten würde. Aber selbst zur Zeit des Völkerbundes, in einer Phase, in welcher der kollektivrechtliche Minderheitenschutz große Unterstützung erfahren hatte, konnte sich die Staatengemeinschaft nicht auf eine Definition einigen. Die Formulierung des Minderheitenschutzes in Artikel 27 IPbpR von 1966 offenbart das Konfliktfeld zwischen individualrechtlichen Ansätzen und einer kollektiv-rechtlichen Ausgestaltung. ${ }^{329}$

In those states in which ethnic, religious or linguistic minorities exist, persons belonging to such minorities shall not be denied the right, in community with the other members of their group, to enjoy their own culture, to profess and practise their own religion, or to use their own language. 330

Der individualrechtliche Teil der Formulierung, persons belonging to such minorities' löst zwar das Problem der prozessualen Anwendbarkeit, indem es die Rechtsgarantien des Artikels 27 IPbpR auf bestimmbare Individuen konkretisiert. Zur Frage der kollektivrechtlichen Dimension des Minderheitenbegriffs, der notwendigerweise den Hintergrund dieser Norm bildet, konnte allerdings während der Verhandlungen keine Einigung erzielt werden. Und auch bei neueren Instrumenten konnte für eine allgemeingültige Definition kein Konsens gefunden werden. „As to what constitutes a minority, there seems only to be general agreement that there is no generally agreed definition. "331

Dennoch haben sich in der langjährigen Geschichte des internationalen Minderheitenschutzes verschiedene Ansätze einer gewissen Konkretisierung des Minderheitenbegriffs herausgebildet. Neben dem Ständigen Internationalen Gerichtshof befassten sich in der UN zwischen 1971 und 1977 der Sonderberichterstatter Francesco Capotorti, 1985 das UN Sub-Commission-Mitglied Jules Deschênes und 1993 der Sonderberichterstatter Esbjørn Eide mit der Entwicklung einer Arbeitsdefinition. Schließlich fanden diese offiziellen Dokumente auch eine eingehende Bearbeitung in der wissenschaftlichen Literatur, die weitergehende Analysen und Konkretisierungen der Begrifflichkeiten vorantrieb.

\footnotetext{
${ }^{329}$ Siehe dazu „A. I. Geschichte des Minderheitenschutzes im Völkerrecht“ auf S. 14.

330 IPbpR, Art. 27.

331 Thornberry, International law and the rights of minorities, S. 164.
} 


\section{Definitionsansatz des Ständigen Internationalen Gerichtshofs}

Eine der ersten rechtlichen Definitionsansätze von ,Minderheiten“ wurde 1930 vom Ständigen Internationalen Gerichtshof in der Greco-Bulgarian Communities Entscheidung zu Artikel 6 (2) der Convention between Greece and Bulgaria respecting reciprocal emigration erarbeitet. In diesem Kontext ging das Gericht davon aus, die geschützte Gruppe sei

a group of persons living in a given country or locality, having a race, religion, language and traditions of their own, and united by the identity of such race, religion, language and traditions in a sentiment of solidarity, with a view to preserving their traditions, maintaining their form of worship, securing the instruction and upbringing of their children in accordance with the spirit and traditions of their race and mutually assisting one another. ${ }^{332}$

Der StIGH formulierte damit eine Definition, die sich auf ein Element der Distinktion im Sinne von Rasse, Religion, Sprache und Traditionen bezieht und ein Element der Solidarität untereinander sowie einen Willen zur Erhaltung und Weitergabe der eigenen Kultur voraussetzt. Weniger stark betont und konkretisiert ist eine geographische oder historische Verwurzelung der Gruppe auf ihrem Land. Im Sinne des StIGHs konstituiert sich eine Minderheit demnach hauptsächlich in Bezug zu einer gemeinsamen Rasse, Religion, Sprache und Tradition.

II. Arbeitsdefinitionen der Sonderberichterstatter und weitere Entwicklungen in den UN

Francesco Capotorti erarbeitete im Rahmen der Sub-Commission on Prevention of Discrimination and Protection of Minorities in Nachbereitung des Artikel 27 IPbpR eine Konzeption des Minderheitenbegriffs, die für viele Arbeiten im politischen und wissenschaftlichen Kontext den Anknüpfungspunkt für weitergehende Untersuchungen bildete. Nach ihr ist eine Minderheit

a group numerically inferior to the rest of the population of a state, in a non-dominant position, whose members - being nationals of the state - possess ethnic, religious or linguistic characteristics differing from those of the rest of the population and show, if only implicitly, a sense of solidarity, directed

\footnotetext{
332 Permanent Court of International Justice, „Greco-Bulgarian Communities“, 1930 PCIJ, Ser B,
} No. 17, 31. Juli 1930, S. 21. 
towards preserving their culture, traditions, religion or language. 333

Neben dem Element der Distinktion in Form von Ethnizität, Religion oder Sprache und der Komponente des ,Ausdrucks eines Gefühls der Verbundenheit', die auf den Erhalt der Kultur gerichtet ist, werden in dieser Definition die Voraussetzung der Staatsangehörigkeit und der im Verhältnis zum Staatsvolk quantitativen Unterlegenheit und nicht-dominierenden Position formuliert. Trotz dieses konkreten Formulierungsvorschlages seiner Arbeitsdefinition, die starke Verbreitung gefunden hat, konnte die Menschenrechtskommission seinem Aufruf der Erarbeitung einer allgemein anerkannten Definition nicht folgen. Ebenso scheiterte zunächst die Initiative zur Entwicklung einer Minderheitendeklaration zu Artikel 27 IPbpR, die in diesem Zusammenhang auf der 34. Tagung 1978 angestoßen wurde. ${ }^{334}$

Eine Weiterentwicklung der Capotorti-Definition erarbeitete Jules Deschênes in seinem Vorschlag an die UN Sub-Commission von 1985. Eine Minderheit sei demnach

a group of citizens of a state, constituting a numerical minority and in a non-dominant position in that state, endowed with ethnic, religious or linguistic characteristics which differ from those of the majority of the population, having a sense of solidarity with one another, motivated, if only implicitly, by a collective will to survive and whose aim it is to achieve equality with the majority in fact and in law. ${ }^{335}$

Er nutzt für die Beschreibung der zahlenmäßigen Unterlegenheit, die Staatsangehörigkeit und die Bezugsgruppe jeweils andere Begriffe als Francesco Capotorti und macht darüber hinaus das Ziel der Gleichstellung ,in fact and law“ deutlich. ${ }^{336}$ Außerdem setzt er die Minderheit nicht, wie Francesco Capotorti, in Verhältnis zum „,rest of the population“ sondern zur Gruppe der „majority“. Dies eröffnet die Interpretationsmöglichkeit, dass in pluriethnischen Staaten ohne dominierende Mehrheitsbevölkerung eine Minderheit mangels Bezugsgruppe nicht zu bestimmen wäre. Sein Ansatz unterscheidet sich aber im Grunde sonst kaum von dem Francesco Capotortis.

\footnotetext{
333 Sub-Commission on the Prevention of Discrimination and Protection of Minorities, Francesco

Capotorti, ,Study on the rights of persons belonging to ethnic, religious, and linguistic minorities“, UN Doc. E/CN.4/Sub.2/384/Rev.1, Abs. 568.

334 Scherer-Leydecker, Minderheiten und sonstige ethnische Gruppen, S. 80-85.

335 Sub-Commission on the Prevention of Discrimination and Protection of Minorities, Jules

Deschênes, „Proposal concerning a definition of the term ,minority“", UN. Doc.

E/CN.4/Sub.2/1985/31, 14. Mai 1985, Abs. 181.
}

336 Thornberry, International law and the rights of minorities, S. 7. 
Trotz dieser Vorarbeiten musste auch die 1992 ohne Abstimmung angenommene Declaration on the Rights of Persons Belonging to National or Ethnic, Religious and Linguistic Minorities ohne Definition des Minderheitenbegriffs auskommen. ${ }^{337} \mathrm{Im}$ Verlauf der Verhandlungen wurden verschiedene Standpunkte und Einzelheiten kontrovers diskutiert. Dazu zählte unter anderem die Frage, ob indigene Völker hier von dem Begriff ,Minderheit ${ }^{\star}$ umfasst sein sollten, ${ }^{338}$ die Frage nach der Voraussetzung der Staatsangehörigkeit, einer historischen und territorialen Verwurzelung sowie die Frage der numerischen Unterlegenheit. ${ }^{339}$ Die deutsche Delegation beharrte beispielsweise darauf, dass sie als Minderheiten nur ,well-defined separate distinct groups which had been long established in the territory of a State" anerkenne. ${ }^{340}$

Bereits parallel zu den Arbeiten an der Minderbeiten-Deklaration befasste sich die UN Sub-Commission mit Fragen der Implementierung und Durchsetzung des Minderheitenschutzes auf nationaler Ebene und setzte dafür 1989 den Sonderberichterstatter Asbjørn Eide ein. ${ }^{341}$ Dabei legte er sich folgende Arbeitsdefinition zu Grunde:

For the purpose of this study, a minority is any group of persons resident within a sovereign State which constitutes less than half of the population of the national society and whose members share common characteristics of an ethnic, religious or linguistic nature that distinguish them from the rest of the population. ${ }^{342}$

Asbjørn Eides Arbeitsdefinition stellt neben der Voraussetzung des Wohnsitzes und einer gemeinsamen Eigenschaft ethnischer, religiöser oder sprachlicher Natur auf eine explizit quantitative Unterlegenheit der Gruppe gegenüber der nationalen Gesellschaft ab. Die UN Sub-Commission änderte diese zahlenmäßig eindeutige Formulierung für die folgende Initiative zur Einrichtung einer weiterführenden Arbeitsgruppe in „minority groups in society“ ab. Aber trotz dieser Anpassung fand seine Formulierung einer Definition im Zuge der weiteren

337 UN Generalversammlung, ,Declaration on the Rights of Persons Belonging to National or Ethnic, Religious and Linguistic Minorities, UN Doc. A/Res/47/135, 3. Februar 1993.

338 Ablehnend Deschênes: Sub-Commission on the Prevention of Discrimination and Protection of Minorities, Jules Deschênes, „Proposal concerning a definition of the term ,minority', UN Doc. E/CN.4/Sub.2/1985/31, 14. Mai 1985, Abs. 38.

339 Scherer-Leydecker, Minderheiten und sonstige ethnische Gruppen, S. 80-85.

${ }^{340}$ Commission on Human Rights, „Forty-eighth session, Summary Record of the 38th Meeting“, UN Doc. E/CN.4/1992/SR.38, 10. April 1992, Abs. 30.

341 Sub-Commission on the Prevention of Discrimination and Protection of Minorities, Asbjørn Eide, „Possible Ways and Means of Facilitating the Peaceful and Constructive Solution of Problems Involving Minorities“, UN Doc. E/CN.4/Sub.2/1993/34 and Add. 1-4, 10. August 1993, Abs. 11.

342 Ebd., Abs. 29. 
Überarbeitung durch die Human Rights Commission keinen Eingang in die Vorschläge an den ECOSOC.343

\section{Konkretisierung durch den Menschenrechtsausschuss}

Der UN Menschenrechtsausscbuss, der aus 18 unabhängigen Experten besteht und der im Rahmen des IPbpR Staatenberichte und Individualbeschwerden auch zum Minderheitenschutz behandelt, äußerte sich nur im Rahmen seines general comment zu Artikel 27 IPbpR zur Frage der Definition des Minderheitenbegriffs. Die comments zu den Staatenberichten und die views zu den Individualbeschwerden enthalten bis auf eine kurze Bemerkung in einer dissenting opinion im Fall Ballantyne et al. $v$ Canada ${ }^{344}$ keine näheren Ausführungen zur Frage der Definition. ${ }^{345}$ Durch die Anwendung des Artikels 27 IPbpR auf Minderheitengruppen in Afrika und indigene Völker zeigt sich aber implizit das weite Verständnis des Minderheitenbegriffs des Menschenrechtsausschusses. ${ }^{346}$

Der general comment führt zur Frage des Minderheitenbegriffs des Artikels 27 IPbpR Folgendes aus:

Article 27 confers rights on persons belonging to minorities which 'exist' in a State party. Given the nature and scope of the rights envisaged under that article, it is not relevant to determine the degree of permanence that the term 'exist' connotes. Those rights simply are that individuals belonging to those minorities should not be denied the right, in community with members of their group, to enjoy their own culture, to practise their religion and speak their language. Just as they need not be nationals or citizens, they need not be permanent residents. Thus, migrant workers or even visitors in a State party constituting such minorities are entitled not to be denied the exercise of those rights. As any other individual in the territory of the State party, they would, also for this purpose, have the general rights, for

\footnotetext{
343 Commission on Human Rights, „Report of the Sub-Commission on the Prevention of Discrimination and Protection of Minorities on its Forty-Sixth Session“, UN Doc. E/CN.4/1995/2, 28. Oktober 1994, S. 11f.

344 Ballantyne et al. v. Canada, UN Doc. A/48/40(PARTII), S. 91.

345 Siehe dazu sogleich „A. V. 2. Quantitative oder politische Inferiorität“ auf S. 89.

346 In ihrem Sondervotum spricht sich die Expertin Elisabeth Evatt gegen die Definition von Minderheiten aufgrund rein numerischer Inferiorität aus. Ballantyne et al. v. Canada, UN Doc. A/48/40(PARTII), 1. November 1993, S. 109; Die Anwendung von Art. 27 IPbpR auf Indigenous Peoples findet sich beispielsweise in: Lovelace v. Canada, UN Doc. A/36/40 (Supp), 29. September 1981, Annex XVIII, S. 166; und in: Lubicon Lake Band v. Canada, UN Doc. A/45/40(Vol.II)(Supp), 4. Oktober 1990, Annex IX.A., S. 1; Zur Anwendbarkeit von Art. 27 IPbpR in afrikanischen Staaten (bspw. Burundi, Niger und Ägypten) s. m.w.N. Scherer-Leydecker, Minderbeiten und sonstige ethnische Gruppen, S. 93.
} 
example, to freedom of association, of assembly, and of expression. $[\ldots]^{347}$

Wie sich bereits in seinen comments und views zeigt, legt der Menschenrechtsausschuss ein weites Verständnis des Minderheitenbegriffs für die Anwendung des Artikels 27 IPbpR zu Grunde. Er schließt explizit die Einschränkung einer notwendigen Staatsangehörigkeit ebenso wie eine historische oder geographische Verwurzelung oder den notwendigen Wohnsitz aus, so dass auch Wanderarbeiter oder sogar Besucher eines Staates eine Minderheit sein können. Ohne eine eigentliche Definition zu formulieren konstituieren sich Minderheiten im Verständnis des Menschenrechtsausschusses anhand der gemeinsamen Kultur, Religion und Sprache.

IV. Regionale Konkretisierung der Minderheitendefinition

Auch auf regionaler Ebene wurde keine feste Beschreibung des Konzepts normiert. So enthält die Framework Convention for the Protection of National Minorities (FCNM) des Europarats, wie die meisten multilateralen Verträge zum Minderheitenschutz, keine allgemein anwendbare Definition. Allerdings lassen sich, so argumentiert Björn Arp, aus dem Zusammenspiel von softlaw und der European Charter for Regional or Minority Languages sowie der Vielzahl von bilateralen Verträgen in Europa die Schlüsselelemente einer Definition durch Auslegung ermitteln. ${ }^{348}$ Dies sind insbesondere die Voraussetzung der Staatsangehörigkeit, eine traditionelle oder historische Verwurzelung auf dem Staatsgebiet des Heimatstaates, ein Element des Zugehörigkeitsgefühls zur Gruppe sowie ein Distinktionsmerkmal vom Rest der Bevölkerung. ${ }^{349}$ Auch hier tauchen die Elemente der Definition Capotortis wieder auf, wobei der Anwendungsbereich durch die Voraussetzungen der Staatsbürgerschaft und historischen und geographischen Verwurzelung eingeschränkt wird.

\section{Verschiedene Elemente einer Minderheitendefinition}

In den verschiedenen Definitionsansätzen in der Praxis und in den Diskussionen in der Literatur ${ }^{350}$ kehren einige Elemente, die eine Minderheitendefinition ausmachen könnten, immer wieder. In unterschiedlichen Konstellationen und mit unterschiedlicher Gewichtung sind dies: das Element der kulturellen, religiö-

\footnotetext{
347 Human Rights Committee, „General Comment No. 23: The rights of minorities (Art. 27)“, CCPR/C/21/Rev.1/Add.5, Abs. 5.2.

348 Arp, International norms and standards for the protection of national minorities, S. 24-31.

349 Ebd., S. $30 f$.

350 Umfassende Monographien zum Minderheitenschutz finden sich von: Scherer-Leydecker, Minderheiten und sonstige ethnische Gruppen; Roßkopf, Theorie des Selbstbestimmungsrechts und Minderheitenrechts; Arp, International norms and standards for the protection of national minorities.
} 
sen, ethnischen oder sprachlichen Distinktion, der Ausdruck eines Zusammengehörigkeitsgefühls der Mitglieder der Gruppe, eine Bezeichnung der unterlegenen Stellung der Gruppe im Staat, die Frage der Staatsangehörigkeit sowie das Element der historischen oder geographischen Verwurzelung oder des Wohnsitzes. ${ }^{351}$ Bis auf den letztgenannten Aspekt sind dies die Elemente der Definition, die bereits Francesco Capotorti zur Grundlage seiner Untersuchung gemacht hat, der als ,realistischer ${ }^{6}$ Ansatz starke Verbreitung gefunden hat. ${ }^{352}$ Umfassend vorbehaltlose Zustimmung oder positivrechtliche Verankerung hat auch sie in der langen Geschichte des Minderheitenrechts allerdings nicht gefunden.

In der Literatur bildete sich aus den verschiedenen Elementen ein weites Spektrum unterschiedlicher Ansätze, in denen verschiedene Problemkonstellationen verortet sowie entsprechende Lösungsvorschläge unterbreitet und begründet werden. So empfiehlt beispielsweise Eckert Klein, bereichsspezifische Definitionen zu entwickeln. ${ }^{353}$ Geht es um bloße Zurückhaltung des Staates, wie beim Diskriminierungsverbot, der Religionsfreiheit oder der Ausübung der Kultur im privaten Raum, sei ein breiter Minderheitenbegriff akzeptabel. Für diese Rechte wäre eine Definition angebracht, wie ihn beispielsweise der UN Menschenrechtsausschuss in seinem general comment zu Artikel 27 IPbpR anlegt. ${ }^{354}$ Handelt es sich um Verpflichtungen zu positiven Fördermaßnahmen, wie der Förderung von Schulunterricht oder die Einführung von zusätzlichen Amtssprachen, sollte eine enge Definition Anwendung finden, um die Belastungen für den Staat zumutbar zu halten. ${ }^{355}$ Eckart Klein hält diese Unterscheidung für angebracht, um Enttäuschungen vorzubeugen. Bewertungsmaßstab seines Vorschlages einer Definition ist somit die Perspektive einer Rechtsfolgenabschätzung, die insbesondere die wirtschaftlichen Auswirkungen des Minderheitenschutzes in den Blick nimmt.

Unabhängig vom Blick auf die Rechtsfolgen, von der Gewichtung der einzelnen Elemente zueinander und der Frage der kumulativen Anwendbarkeit der einzelnen Elemente soll den einzelnen Aspekten in ihrer Aussagekraft zur Bestimmung substaatlicher Gruppen nachgegangen werden.

\footnotetext{
351 Vgl. die Auflistung der verschiedenen Elemente einer Definition bei John Packer, The protection of ethnic and linguistic minorities in Europe, 1997, S. 53ff; Letschert, The impact of minority rights mechanisms, S. $28 \mathrm{ff}$.

352 Thornberry, International law and the rights of minorities, S. 6.

353 Eckart Klein, „Die Frage der Minderheitendefinition“, in: Manfred Mohr (Hrsg.), Friedenssichernde Aspekte des Minderheitenschutzes in der Ära des Völkerbundes und der Vereinten Nationen in Europa, 1996.

354 Ebd., S. 211; Human Rights Committee, „,General Comment No. 23: The rights of minorities (Art. 27)“, CCPR/C/21/Rev.1/Add.5, Abs. 5.1.

355 Klein, „Die Frage der Minderheitendefinition“, S. 211.
} 


\section{Kulturelle Distinktion und ein ,Ausdruck des Gefühls der Verbundenheit}

Kern aller Definitionsansätze ist die Unterscheidung der Gruppe aufgrund einer Distinktion in Form von Rasse, Ethnizität, Religion, Sprache oder Tradition sowie eines entsprechenden ,Ausdrucks des Gefühls der Verbundenheit', wie es Francesco Capotorti nannte. So beschreibt Artikel 27 IPbpR explizit seinen Anwendungsbereich für ethnische, religiöse und sprachliche Minderheiten und garantiert für diese Gruppen entsprechend das Recht auf die eigene Kultur, Religion und Sprache. Die Begriffe Rasse und Ethnizität werden heute zunehmend durch die Bezeichnung einer gemeinsamen Kultur und gemeinsamen kollektiven Identität ersetzt. ${ }^{356}$ Des Weiteren wird normiert, dass die Gruppe den Willen zum Ausdruck bringen muss, als zusammengehörig verstanden zu werden, ihre Kultur zu pflegen und nachfolgenden Generationen weiter geben zu wollen. Diese beiden Aspekte der Definition werden oft als objektives und subjektives Element beschrieben und bilden grundsätzlich in jeder Auseinandersetzung mit der Frage der Minderheitendefinition den Ausgangspunkt weiterer Erwägungen.

\section{Quantitative oder politische Inferiorität}

Das Merkmal der Unterlegenheit ist bereits begrifflich in der Bezeichnung, Minderheit' enthalten. Die Unterlegenheit der Gruppe im Verhältnis zur Mehrheit der Gesellschaft eines Staates begründet die Schutzbedürftigkeit und somit Notwendigkeit des Minderheitenschutzes. Sie soll entweder rein numerisch bestimmt oder nach qualitativen Gesichtspunkten bewertet werden. Auch wenn die zahlenmäßige Bestimmung als objektive Methode zu eindeutigen Ergebnissen führt, kann diese problematisch sein. Dies zeigt das Beispiel des Apartheidregimes in Südafrika, in dem die numerische Minderheit die politische Macht inne hatte und eine rein numerische Differenzierung bei der Anwendung des Minderheitenschutzes zu falschen Ergebnissen geführt hätte. ${ }^{357}$

Eine numerische Bestimmung kann auch bei bundesstaatlichen Strukturen zu unangemessenen Ergebnissen führen. ${ }^{358} \mathrm{Da}$ Artikel $50 \mathrm{IPbpR}$ den Pakt auf gesamtstaatlicher Ebene für anwendbar erklärt, ist Artikel $27 \mathrm{IPbpR}$ ebenfalls auf den Gesamtstaat zu beziehen. So argumentierte das Human Rights Committee in seiner Entscheidung im Fall Ballantyne et al. $v$ Canada, dass die englischsprachigen Kanadier in Quebec keine Minderheit im Sinne des Artikel 27 IPbpR darstellen. 359

\footnotetext{
356 John Packer, „Problems in Defining Minorities“, in: Deirdre Fottrell (Hrsg.), Minority and group rights in the new millennium, 1999.

357 Bronwen Manby, „South Africa: minority conflict and the legacy of minority rule“, The Fletcher forum of world affairs, Vol. 19, Nr. 1 (1994), S. 32.

358 Dies problematisiert Elisabeth Evatt in ihrem Sondervotum: Ballantyne et al. v. Canada, UN Doc. A/48/40(PARTII), 1. November 1993, S. 109.

359 Ebd., Abs. 11.2, S. 102.
} 
Die numerische Differenzierung führt somit zwar zu eindeutigen Ergebnissen, verlagert allerdings die Problematik auf die Definition der Vergleichsmehrheitsgesellschaft. Sie kann daher in Einzelfällen einer ungleichen Machtverteilung, oder bei bestimmten numerischen Konstellationen in bundesstaatlichen Gefügen, zu falschen Ergebnissen führen.

\section{Staatsangehörigkeit}

Die Voraussetzung der Staatsangehörigkeit beschreibt zwar nicht die Gruppe in ihrer Eigenschaft als Minderheit, sie gibt aber den Staaten die Möglichkeit, über die Regelungen der Staatsbürgerschaft sowie durch Ein- und Ausbürgerungen den Personenkreis des Minderheitenschutzes zu regulieren. Diese Einflussnahmemöglichkeit über die Politik des Staatsbürgerschaftsrechts wird kontrovers beurteilt. So positioniert sich beispielsweise das Human Rights Committee in seinem general comment gegen diese Voraussetzung, wenn es schreibt, dass

[t]he terms used in article 27 indicate that the persons designed to be protected are those who belong to a group and who share in common a culture, a religion and/or a language. Those terms also indicate that the individuals designed to be protected need not be citizens of the State party. [...] A State party may not, therefore, restrict the rights under article 27 to its citizens alone. ${ }^{360}$

Die regionalen Minderheitenschutzinstrumente in Europa hingegen setzen die Staatsangehörigkeit explizit voraus. ${ }^{361}$

Die Bedeutung der Staatsangehörigkeit im Kontext des Minderheitenschutzes können jüngste Entwicklungen in Ungarn verdeutlichen. Das EU Mitglied Ungarn erlaubt ungarischen Minderheiten, die im Ausland leben und demnach nicht die ungarische Staatsangehörigkeit besitzen, seit dem 1.1.2011 einen ungarischen Pass für eine doppelte Staatsbürgerschaft zu erwerben, ohne dafür eine spezielle Verbindung zu Ungarn in Form eines Wohnsitzes vorweisen zu müssen. Rechtlich interessant ist die ungarische Staatsangehörigkeit besonders für die ungarischen Minderheiten in Serbien (300.000 Mitglieder) und in der Ukraine (150.000 Mitglieder), die somit Zugang zur EU erhalten können. Für die Angehörigen der ungarischen Minderheiten in Rumänien (1,4 Millionen Mitglieder) und der Slowakei (500.000 Mitglieder) hat solch eine doppelte Staatsbürgerschaft eigentlich nur symbolischen Wert, da sie bereits EU-Bürger sind und somit bereits eine rechtli-

${ }^{360}$ Human Rights Committee, „General Comment No. 23: The rights of minorities (Art. 27)“, CCPR/C/21/Rev.1/Add.5, Abs. 5.1.

361 Arp, International norms and standards for the protection of national minorities, S. 24-28; Patrick Thornberry, „International and European standards on minority rights“, in: Hugh Miall (Hrsg.), Minority rights in Europe: the scope for a transnational regime, 1994, S. 18. 
che Gleichstellung genießen. Außerdem garantiert ein bilaterales Abkommen zwischen Ungarn und der Slowakei von 1995 die rechtliche Gleichstellung der Mitglieder der jeweiligen Minderheit mit eigenen Staatsangehörigen, so dass Mitglieder der ungarischen Minderheit in der Slowakei formell dieselben Rechte und Pflichten haben, wie slowakische Staatsbürger. ${ }^{362}$

Die Entwicklungen in der Slowakei verdeutlichen jedoch, dass den Regelungen zur Staatsangehörigkeit für Heimatstaaten, Minderheiten und die Staaten ihrer kulturellen Wurzeln eine Bedeutung zukommt, die über die bloße rechtliche $\mathrm{Zu}$ ordnung von Bürgern zu einem Staat hinausgeht. In der Slowakei wurde in Reaktion auf die ungarische Initiative zur möglichen doppelten Staatsbürgerschaft eine Regelung erlassen, nach der Slowaken, die eine andere Staatsbürgerschaft erhalten, ihren slowakischen Pass verlieren. Mitglieder der ungarischen Minderheit, welche die Möglichkeiten des neuen ungarischen Gesetzes nutzen möchten, würden damit automatisch ihre slowakische Staatsangehörigkeit verlieren. Zwar möchte die neu gewählte Premierministerin Iveta Radičová das Gesetz ihres Vorgängers wieder abschaffen, grundsätzliches Einverständnis mit der Initiative Ungarns zeigt sie allerdings ebenfalls nicht. ${ }^{363}$ Vergleicht man nun die Reaktionen der ungarischen Minderheit in der Slowakei mit denen der ungarischen Minderheit in Rumänien, zeigt sich, dass in Rumänien das Interesse an der ungarischen Staatsbürgerschaft erheblich größer war als in der Slowakei, ${ }^{364}$ und dies, obwohl es durch den europäischen Rahmen für die ungarischen Minderheiten in der Slowakei eigentlich keine rechtlichen Nachteile bedeutet, die Staatsangehörigkeit zu wechseln. Auch wenn sich über die individuellen Motive zum Wechsel oder Nicht-Wechsel der Staatsangehörigkeit der ungarischen Minderheiten im Ausland hier nur spekulieren lässt, zeigt das Beispiel, dass die Frage der Staatsbürgerschaft von Minderheiten im politischen Diskurs erhebliche Debatten erzeugen kann. Zudem verdeutlicht es, dass Staaten mit den Regelungen zum Erwerb und Verlust der Staatsangehörigkeit erheblichen Einfluss auf die Identität der Minderheit nehmen können.

Die Voraussetzung der Staatsangehörigkeit des Heimatstaates für einen Minderheitenstatus eröffnet einen erheblichen Spielraum für staatliche Einflussnahme. So kann über die Regelungen zur Einbürgerung von Immigranten gleichzeitig der Minderheitenstatus verweigert werden. Auch bei etablierten Minderheitengruppen können Regelungen zum möglichen Verlust des Passes des Heimatstaates erheblichen politischen Druck auf die Gruppe ausüben. Das Element der Staatsangehörigkeit im Gefüge einer Minderheitendefinition ist ein Instrument zur regulieren-

\footnotetext{
362 „Treaty on Friendship and Cooperation between the Republic of Hungary and the Republic of Slovakia“, Paris, 19. März 1995, in: Björn Arp (Hrsg.), International Norms and Standards for the Protection of National Minorities, 2008, S. 348.

363 „Slovakia-Hungary row over citizenship law“, EurActiv.com, 27. Januar 2011.

364 „Interesse an ungarischen Pässen“, Frankfurter Allgemeine Zeitung, 15. Februar 2011.
} 
den Einschränkung des Minderheitenstatus' von staatlicher Seite. Eine Konkretisierung, was eine Minderheit definiert, ist diese Voraussetzung jedoch nicht.

\section{Historische und geographische Verwurzelung}

Wie die Voraussetzung der Staatsangehörigkeit wird auch das Element einer historischen und geographischen Verwurzelung aus politischen Motiven zur Einschränkung des Minderheitenschutzes herangezogen. ${ }^{365}$ So versuchte beispielsweise Deutschland in den Verhandlungen zum Mandat des UN Sonderberichterstatters Joseph Voyame die Untersuchung der Situation der Roma und Sinti auf solche Länder zu beschränken, in denen sie traditionell leben. ${ }^{366}$ Dies hätte eine Untersuchung der Anwendbarkeit des Minderheitenrechts der Sinti und Roma in Deutschland ausgeschlossen. ${ }^{367}$ Als diese Forderung jedoch keine Unterstützung fand, erklärte der deutsche Vertreter explizit, dass Roma und Sinti in Deutschland keinen Minderheitenstatus genießen. ${ }^{368}$

Die Voraussetzung der historischen und geographischen Verwurzelung wird auch in der Literatur unterschiedlich beurteilt. ${ }^{369}$ Einerseits wird eine Differenzierung zwischen ,alten' und ,neuen' Minderheiten vorgenommen. Die ,alten' Minderheiten sollen dabei durch Gruppenrechte und klar umrissene Verpflichtungen von Seiten des Staates geschützt werden, während ,neue' Minderheiten sich nur auf den Grundsatz der Nichtdiskriminierung berufen können. ${ }^{370}$ Andererseits wird vertreten, dass in modernen multi-ethnischen Staaten sowohl ,alte ${ }^{6}$ als auch ,neue Minderheiten besonderen Minderheitenschutz von Seiten des Staates genießen. ${ }^{371}$ Auch hier unterscheiden sich somit die Meinungen vor dem Hintergrund entsprechender politischer Erwägungen im Umgang mit Minderheiten und Migranten.

\section{Zusammenfassung}

Die Untersuchung der Definitionsansätze des Konzepts ,Minderheit“ zeigt, dass sich unterschiedliche Ausgestaltungen jeweils institutionell und politisch verorten lassen. Das Expertengremium des UN Menschenrechtsausschusses legt seiner Arbeit beispielsweise einen sehr weiten Minderheitenbegriff zu Grunde, um dem Interesse einer verbreiteten Anwendung des Instruments und der eigenen Zuständigkeit nachzukommen. Die multilateralen Verträge in Europa zeigen ein

\footnotetext{
365 Arp, International norms and standards for the protection of national minorities, S. 29f.

366 Commission on Human Rights, „Summary Record of the 54th Meeting“, UN Doc.

E/CN.4/1992/SR.54, 16. April 1992, Abs. 3.

367 Scherer-Leydecker, Minderheiten und sonstige ethnische Gruppen, S. 86.

${ }^{368}$ Commission on Human Rights, „Summary Record of the 54th Meeting“, UN Doc.

E/CN.4/1992/SR.54, Abs. 19.

${ }^{369}$ Nowak, „The Evolution of Minority Rights in International Law, Comments“, S. 116.

370 Ebd.

371 Ebd.
} 
enges Verständnis des Minderheitenbegriffs und setzen zudem die Staatsangehörigkeit des Heimatstaates voraus. Die Mitgliedstaaten dieser Verträge möchten den Anwendungsbereich begrenzen, um nicht mehr Gruppen als nötig die niedergelegten Minderheitenrechte einräumen zu müssen. In der Literatur werden die Definitionen in einer Gesamtschau mit einem selektiven Ansatz zu den Rechtsfolgen diskutiert. Aus dieser Gesamtschau ergibt sich dann, wem man welche Rechte gewähren sollte. Diese Kombinationsmöglichkeiten lassen sich ergebnisorientiert nach individuell politischen Zielen gestalten. Ob eine Gruppe eine Minderheit darstellt, obliegt also vielmehr der individuellen Anwendung je eigener Definitionsansätze der verschiedenen Gremien, Institutionen und Akteure. Dabei ist es eine politische Entscheidung, die Definition entsprechend zu erweitern oder zu konkretisieren, um bestimmte Gruppen als Minderheit anzuerkennen oder, wie dies das Beispiel Deutschlands zeigt, Gruppen explizit vom Minderheitenstatus auszuschließen.

Zusammenfassend lässt sich festhalten, dass die Definitionsansätze im Kern auf eine kulturelle Distinktion der Gruppe gegenüber der Mehrheit eines Staates rekurrieren, die sich durch einen Ausdruck des Gefühls der Verbundenheit der Gruppe zeigt. Außerdem zeichnen sich Minderheiten durch eine numerische oder politische Unterlegenheit im Kontext ihrer Heimatstaaten aus. Des Weiteren werden die Voraussetzungen der Staatsangehörigkeit sowie eine historische und geographische Verwurzelung gefordert, um den Anwendungsbereich des Minderheitenschutzes aus politischen Gründen einzuschränken. 


\section{B. Indigene Völker}

Mit der verstärkten Präsenz von Vertretern indigener Gruppen auf der internationalen Bühne seit Beginn der 1970er Jahre werden Ansätze einer rechtlich verbindlichen Definition des Begriffs ,indigene Völker` diskutiert. Problematisch sind dabei die Implikationen der Selbstbezeichnung indigener Gruppen als ,Volk', da dieser Terminus in seiner Bedeutung explizit an den völkerrechtlich geprägten Begriff des Artikels 1(2) UNO Charta und Artikel 1 IPbpR anknüpft.

\section{Implikationen des Konzepts ,people}

Der Begriff indigenous peoples ist problematisch, weil er nicht nur die Frage des ,Was konstituiert Indigenität ${ }^{`}$ und dem daraus folgenden ,Wer ist indigen' aufwirft, sondern gleichzeitig auch Aussagen zum Rechtsumfang, zum Rechtsträger und zur Durchsetzung von Rechten beinhaltet. Insbesondere die Bedeutung des Begriffs ,Volk', verstanden im Sinne von Artikel 1 (2) UNO Charta, mit den Implikationen des Rechts auf Selbstbestimmung und dem Recht auf Sezession, öffnet Interpretationsspielräume von erheblicher Tragweite.

Nearly fourty years ago, a Professor of Political Science, who was also President of the United States, President Wilson, enunciated a doctrine which was ridiculous, but which was widely accepted as a sensible proposition, the doctrine of self-determination. On the surface it seemed reasonable: let the people decide. It was in fact ridiculous because people cannot decide until someone decides who the peoples are..$^{372}$

Die Indigenen verknüpften ihre politischen Forderungen mit der Selbstbezeichnung als ,Volk' und schöpften somit politisches Potential aus den normativen Wertungen der Zeit der Neuordnung Europas und der Dokolonisation. Dementsprechend erfuhr das Konzept der, indigenen Völker' erhebliche Opposition von Seiten der Staaten, so dass die Diskussionen anfangs von staatlicher Seite unter dem politisch und rechtlich weniger geprägten Begriff ,population' geführt wurden. ${ }^{373}$

Im Zuge der jüngsten Entwicklungen versteht man die Debatte nunmehr im Lichte der menschenrechtlichen Dimension, in deren Kontext die Diskurse um die Rechte indigener Völker mittlerweile stattfinden. Darin wird das Recht auf Selbstbestimmung, wie es schließlich 2007 in der UN Declaration on the Rights of Indigenous Peoples auch explizit Ausdruck fand, als grundlegender Begriff anders

\footnotetext{
372 Zitat von Ivor Jennings in: Castellino, International law and self-determination, S. 16.

373 Augusto Willemsen Diaz, „How Indigenous Peoples’ Rights reached the UN“, S. 24.
} 
verstanden. Es wird insbesondere zwischen dem durch den Prozess der Dekolonisation geprägten Verständnis als Sezessionsrecht und Recht auf Staatsgründung, was nur die außergewöhnlichste Form der Durchsetæung ist, und dem eigentlich Inhalt des Rechts getrennt. Inhaltlich umfasst das Recht auf Selbstbestimmung, als ein Volk die eigene politische Ordnung und die Ausgestaltung der regierenden Institutionen konstituieren und verändern zu können sowie fortwährend im Rahmen der gegebenen Institutionen bedeutende Entscheidungen, die sich auf alle Bereiche des Lebens auswirken, selbst auszuhandeln. ${ }^{374}$ Davon zu differenzieren ist die Form der Durchsetzung, die nur in Ausnahmefällen zu Sezession und Staatsgründung führen kann. ${ }^{375}$

\section{Die Definition von José Martínez Cobo}

Lässt man die Fragen der inhaltlichen Bedeutung und der Durchsetzungsmöglichkeiten des Selbstbestimmungsrechts indigener Völker beiseite, ist Ausgangspunkt einer völkerrechtlichen Antwort auf die Frage, was und wer indigen ist, zunächst der erhobene Anspruch indigener Völker, selbst bestimmen zu lassen, was unter dem Begriff zu verstehen ist. ${ }^{376}$ Auch das abschließende Kapitel des Berichts des UN Sonderberichterstatters José Martínez Cobo von 1983, 377 welches die grundlegende Arbeit für die weiteren Entwicklungen im Rahmen der UN bildete, stellt fest, dass das Recht indigener Völker anerkannt werden muss, selbst zu definieren, was und wer indigen ist. Als grundlegend für weitere Entwicklungen in der Praxis und der Wissenschaft gilt allerdings seine Arbeitsdefinition aus dem genannten Bericht:

Indigenous communities, peoples and nations are those which, having a historical continuity with pre-invasion and pre-colonial societies that developed on their territories, consider themselves distinct from other sectors of the societies now prevailing in those territories, or parts of them. They form at present nondominant sectors of society and are determined to preserve, develop and transmit to future generations their ancestral territories, and their ethnic identity, as the basis of their

\footnotetext{
374 Anaya, Indigenous peoples in international law, S. 104-106; Patrick Thornberry, Indigenous peoples and buman rights, 2002, S. 382; Erica-Irene Daes, ,'The Contribution of the Working Group on Indigenous Populations to the Genesis and Evolution of the UN Declaration on the Rights of Indigenous Peoples“, in: Claire Charters und Rodolfo Stavenhagen (Hrsg.), Making the Declaration Work, the United Nations Declaration on the Rights of Indigenous Peoples, 2009, S. 70.

375 Anaya, Indigenous peoples in international law, S. 109.

376 Monika Ludescher, Menschenrechte und indigene Völkeer, 2004, S. 348.

377 Sub-Commission on the Prevention of Discrimination and Protection of Minorities, José R. Martínez Cobo, „Study of the Problem of Discrimination against Indigenous Populations“, UN Doc. E/CN.4/Sub.2/1986/7/Add.4, Abs. 369.
} 
continued existence as peoples, in accordance with their own cultural patterns, social institutions and legal systems. ${ }^{378}$

Ausgehend von dieser Definition, die grundlegende Akzeptanz gefunden hat, lassen sich aus völkerrechtlicher Perspektive folgende Unterscheidungsmerkmale differenzieren: eine gewisse Distinktion, ein Solidaritätsgefühl in der Gruppe, eine historische und geographische Verwurzelung in der vorkolonialen Geschichte sowie eine nicht herrschende Stellung in der Gesellschaft. Indigene Völker unterscheiden sich demnach in ethnischer, kultureller, sprachlicher sowie religiöser Hinsicht von den Teilen der Gesellschaft, die heute auf den Gebieten leben, wo einst Indigene vorherrschten. ${ }^{379}$ Diese kulturellen Besonderheiten äußern sich beispielsweise im Stammes- oder Rechtssystem, der Kleidung sowie der Art und Weise der Erwirtschaftung des Lebensunterhalts. Diese kulturellen Besonderheiten sind dabei auch Inhalt des kollektiven Identitätsgefühls in dessen Sinne die eigene Lebensweise erhalten und in nachfolgende Generationen weitergetragen werden möchte. Mitglied einer indigenen Gruppe ist demnach, wer sich als indigene Person identifiziert, das entsprechende Gruppenbewusstsein besitzt und als solche oder solcher von der Gruppe als Mitglied anerkannt ist. Die Entscheidungsbefugnis, wer konkret Mitglied der Gruppe wird, bleibt demnach der indigenen Gemeinschaft selbst überlassen.

Das Element der nicht herrschenden Stellung bezieht sich auf eine historische oder gegenwärtige politische Unterlegenheit sowie eine soziale und wirtschaftliche Benachteiligung. ${ }^{380}$

Das Element der historischen und geographischen Verwurzelung ergibt sich aus einem oder mehreren der folgenden Faktoren, wie Martínez Cobo dies in seinem Bericht differenziert: „occupation of ancestral land, common ancestry with the original occupants of the land, culture in general, or in specific manifestations, language, residence in certain parts of the country“.381 Die historische Kontinuität wird als lineare Verbindung zu Gesellschaften vor der Invasion und Kolonisierung durch die Europäer verstanden. Wichtig ist ebenso die Verbindung indigener Völker zu ihrem Land, welches nicht nur in rein wirtschaftlicher, sondern insbesondere auch in spiritueller Hinsicht als Lebensgrundlage dient. ${ }^{382}$ Indigenität bezeichnet also eine kulturelle Andersartigkeit vor dem Hintergrund der Kolonisierung und

\footnotetext{
378 Ebd., Abs. 379.

${ }^{379}$ Ludescher, Menschenrechte und indigene Völkeer, S. 351.

380 Chris Tennant, „Indigenous Peoples, International Institutions, and the International Legal Literature from 1945-1993“, in: Anthony J Connolly (Hrsg.), Indigenous Rights, 2009, S. XV.

381 Sub-Commission on the Prevention of Discrimination and Protection of Minorities, José R. Martínez Cobo, „Study of the Problem of Discrimination against Indigenous Populations“, UN Doc. E/CN.4/Sub.2/1986/7/Add.4, Abs. 380.

382 Catherine Brölmann und Marjoleine Zieck, „Indigenous Peoples“, in: Catherine Brölmann, René Lefeber, und Marjoleine Zieck (Hrsg.), Peoples and Minorities in International Law, 1993, S. 193.
} 
Unterdrückung, die des Schutzes vor den Entwicklungen der Modernisierung, Säkularisierung, Urbanisierung und ,Verwestlichung' bedarf. ${ }^{383}$

\section{Definitionen der ILO Konventionen}

Die erste völkerrechtliche Konvention, die sich explizit mit der Situation von Indigenous and Tribal and Semi-Tribal Populations befasste, ILO Convention No. 107 von 1957, beinhaltet in ihrem Artikel 1 eine Begriffsbestimmung. ${ }^{384}$ Im Lichte der damaligen Intention der Integration und Entwicklung indigener Gruppen bezieht sich auch die Definition auf die Aspekte der, weniger fortschrittlichen sozialen und ökonomischen Verhältnisse' der Gruppen, deren Status ganz oder zum Teil durch eigene Gebräuche und Traditionen oder gesonderte rechtliche Regelungen bestimmt wird. Des Weiteren wird eine Beschreibung formuliert, welche die Mitglieder bezüglich ihrer Verbindung zu vorkolonialen Gesellschaften bestimmt, ,deren Lebensweise eher den sozialen, ökonomischen und kulturellen Institutionen damaliger Zeit als den staatlichen Institutionen entspricht'. ${ }^{385}$ In Anbetracht der aus der Konvention sprechenden Assimilationspolitik, der mangelnden Beteiligung indigener Gruppen bei der Erarbeitung des Dokuments und der geringen Zahl von nur 17 Ratifikationen konnte ihr Definitionsansatz grundsätzlich keine umfassende Zustimmung erreichen.

Die Folgekonvention ILO Convention No. 169 von 1989 ist eine Überarbeitung ihres Vorgängerdokuments und zeigt deutlich den veränderten Zeitgeist, der schon in der Studie von José Martínez Cobo zu erkennen war. Ihr Artikel 1 definiert ihren Anwendungsbereich für:

(a) tribal peoples in independent countries whose social, cultural and economic conditions distinguish them from other sections of the national community, and whose status is regulated wholly or partially by their own customs or traditions or by special laws or regulations;

(b) peoples in independent countries who are regarded as indigenous on account of their descent from the populations which inhabited the country, or a geographical region to which the country belongs, at the time of conquest or colonisation or the establishment of present state boundaries and who, irrespective of their legal status, retain some or all of their own social, economic, cultural and political institutions. ${ }^{386}$

\footnotetext{
383 Will Kymlicka, „Theorizing Indigenous rights“, University of Toronto law journal, Vol. 49, Nr. 2 (1999), S. 289.

384 ILO Convention No. 107, Art. 1.

385 Ebd., Art. 1 (b).

386 ILO Convention No. 169, Art. 1.
} 
Es findet der Begriff peoples Verwendung, ${ }^{387}$ es wird neutral auf soziale, kulturelle und ökonomische ,Unterschiede“ und nicht die dahingehende, mangelnde Fortschrittlichkeit' abgestellt und lit. (b) fügt neben der Voraussetzung der Verbindung zu vorkolonialen Gesellschaften eine Variante des zeitlichen Horizont der ,Grenzziehung heutiger Staatsgrenzen‘ hinzu. Des Weiteren wird in Absatz 2 die grundsätzliche Bedeutung der Selbstidentifikation deutlich:

Self-identification as indigenous or tribal shall be regarded as a fundamental criterion for determining the groups to which the provisions of this Convention apply.

Die ILO Convention No. 169 überwindet nicht nur den vordergründigen Tenor der Assimilationspolitik ihrer Vorgängerin, sondern folgt mit der Verwendung der Bezeichnung peoples und der Betonung der self-identification as indigenous or tribal zwei der Kernforderungen indigener Völker. Dieser Definitionsansatz setzte sich allerdings nicht global durch. Die 22 Ratifikationen liegen vornehmlich in Südamerika und reflektieren die mangelnde globale Zustimmung von Seiten der Staaten. ${ }^{388}$ Vertreter indigener Völker kritisierten anfangs, dass die Konvention ein Über-/Unterordnungsverhältnis zwischen Staat und indigenen Gruppen impliziere und drückten somit ihre Unzufriedenheit über den Verhandlungsverlauf aus, bei dem sie nur beratend hinzugezogen wurden. ${ }^{389}$ Auch seien der Aspekt der Selbstbestimmung und der kollektivrechtliche Charakter der Regelungen nicht stark genug verankert worden. ${ }^{390}$ Die Auseinandersetzungen bezüglich dieser Aspekte, die sich vornehmlich um die Verwendung des Begriffs peoples drehten, machten Kompromisse auf Seiten der Staaten erforderlich, welche sich schließlich in den ausstehenden Ratifikationen in Afrika und Asien niederschlagen. ${ }^{391}$

IV. Regionale Interpretationen in Asien und Afrika

In Anbetracht der globalen Indigenenbewegung, im Zuge derer sich indigene Völker auch in Asien und Afrika finden, bereitet die Subsumtion dieser Gruppen aus Nicht-Siedlergesellschaften unter die Definitionen von José Martínez Cobo Probleme. Die Staaten Asiens und Afrikas argumentieren dahingehend, dass entweder alle oder niemand ihrer Staatsbürger indigen seien und das Kon-

387 Dahingehend wird allerdings in Absatz 3 deutlich gemacht, dass ,, $\mathrm{t}]$ he use of the term peoples in this Convention shall not be construed as having any implications as regards the rights which may attach to the term under international law."

388 Maria Victoria Cabrera Ormaza, „Re-thinking the Role of Indigenous Peoples in International Law: New Developments in International Environmental Law and Development Cooperation", Goettingen Journal of International Law, Vol. 4, Nr. 1 (2012), S. 273.

389 Anaya, Indigenous peoples in international law, S. 59.

390 Ebd.

391 Ebd., S. 60. 
zept der, indigenen Völker ‘ mit den Rechten auf Selbstbestimmung, da sie keine weiße Siedlungsgeschichte wie Nordamerika haben, nicht einschlägig sei. ${ }^{392}$ Entgegen dieser Meinung formieren und organisieren sich Gruppen als indigene Völker Asiens und Afrikas in internationalen NGOs und in Zusammenarbeit mit den etablierten Foren indigener Völker aus Nordamerika, Australien und Neuseeland. Schlüssel ihrer Selbstidentifikation als Indigene entgegen der Definition José Martínez Cobos ist eine Uminterpretation, die den Fokus weniger auf die Distinktion gegenüber einer weißen Siedlergesellschaft, als vielmehr auf eine ähnliche Erfahrung der Marginalisierung und Unterdrückung legt.

In Asien stellen sich die Probleme der Definition und Anwendbarkeit des Begriffs, indigenes Volk' mangels einer historisch gegebenen Trennung von Einwanderergesellschaft und Ureinwohnern. Die Begründung des Konzepts kann hier nicht auf historische Ungerechtigkeiten, Verbrechen und einer daraus resultierenden Notwendigkeit von Entschädigung zurückgeführt werden. ${ }^{393}$ Dennoch findet es Anwendung durch sich selbst als indigen identifizierenden Gruppen und von Dritten, die, wie beispielsweise die Weltbank oder andere internationale Regierungs- und Nichtregierungsorganisationen, globale Konzepte und Definitionen auf eine vergleichbare Interessenlage anwenden. ${ }^{394}$

Auch staatliche Organisationen, wie etwa die African Commission on Human and Peoples' Rights, entwickeln in Anbetracht der regionalen Anwendungsproblematik der dominierenden Definitionsansätze eigene Perspektiven eines Verständnisses des Begriffs ,indigenes Volk' ${ }^{395}$ Grundlage dieser Vorschläge ist dabei, dass indigene Gemeinschaften extreme Formen der Marginalisierung und Diskriminierung erfahren und dass Gruppen, die sich in Afrika selbst als indigen identifizieren, auch sonst vergleichbare Eigenschaften mit der Definition von Indigenität aufweisen: eine kulturelle Unterschiedlichkeit, Selbstidentifikation als kulturell unterschiedlich, eine historische und geographische Verwurzelung in ihrem Land sowie

\footnotetext{
392 Naomi Kipuri, „The UN Declaration on the Rights of Indigenous Peoples in the African Context", in: Claire Charters und Rodolfo Stavenhagen (Hrsg.), Making the Declaration Work, the United Nations Declaration on the Rights of Indigenous Peoples, 2009, S. 256.

${ }^{393}$ Kingsbury, ,„Indigenous Peoples“ in International Law“.

394 Karin Klenke und Philipp Socha, „Emerging Indigeneity - Völkerrechtswissenschaft und ethnologische Praxis subnationaler kultureller Gemeinschaften“, in: Kilian Bizer, Matthias Lankau, und Gerald Spindler (Hrsg.), Sui Generis Recbte zum Schutz, traditioneller kultureller Ausdrucksweisen, Interdisziplinäre Perspektiven, 2013, S. 21-42.

395 African Commission on Human and Peoples' Rights, Advisory Opinion of the African Commission on Human and Peoples' Rights on the United Nations Declaration on the Rights of Indigenous Peoples, 2010, S. 30f. Die Advisory Opinion wurde im Rahmen der 41st ordinary Session der African Commission on Human and Peoples' Rights im May 2007 in Accra, Ghana angenommen; Jérémie Gilbert, „Indigenous peoples' human rights in Africa: the pragmatic revolution of the African Commission on Humand and Peoples' Rights“, International \& Comparative Law Quarterly, Vol. 60, Nr. 1 (2011), S. 245-270.
} 
politische und wirtschaftliche Unterdrückung. ${ }^{396}$ Ausgehend von einer vergleichbaren Interessenlage solcher Gruppen und im Rahmen des Spielraums, der mangels einer formellen Definition gegeben ist, implementieren die African Commission und andere afrikanische Institutionen die Rechte indigener Völker in Afrika.

\section{Die Deklaration der Vereinten Nationen über die Rechte indigener Völker}

Auch die Deklaration der Vereinten Nationen über die Rechte indigener Völker von 2007 beinhaltet keine offizielle Definition des Begriffs, indigenes Volk', da sich im Zuge der Verhandlungen keine allgemein anerkannte Formulierung gefunden hat. ${ }^{397}$ Die Meinungen zur Streichung des zunächst vorgesehenen Artikels 8 des Entwurfs, der eine Definition konkret festschreiben sollte, gehen weit auseinander. Einerseits wird das Fehlen einer klaren Definition als Manko der UNDRIPs gesehen, da sie so weniger präzise sei. Andererseits wird eine Definition als nicht notwendig beziehungsweise als nicht wünschenswert empfunden, da eine zu enge Definition der globalen Anwendbarkeit der Erklärung entgegenstehen könnte. ${ }^{398}$ Insbesondere die afrikanischen und asiatischen Staaten drängten auf eine Definition, um den Interpretationsspielraum einzuengen und somit die ohne Definition möglicherweise als indigen geltenden Gruppen in ihren Ländern aus dem Kreis der Berechtigten auszuschließen. Dabei wurde auch immer wieder geäußert, dass im eigenen Land keine indigenen Völker existierten, vielmehr seien alle Bürger indigen. ${ }^{399}$ Im Ergebnis können sich Gruppen nun weltweit als Indigene identifizieren und an dem politischen Prozess der internationalen Indigenenbewegung teilhaben und mitwirken.

\section{Zusammenfassung}

Zwar spielen indigene Völker bereits seit der Eroberung und Kolonisation der , neuen Welt völkerrechtlich eine Rolle. Mit der zunehmenden Bedeutung der internationalen Indigenenbewegung seit den 1970er Jahren gewann die Frage, wie diese Gruppen in einer abstrakt-generellen Definition völkerrechtlich greif-

\footnotetext{
396 African Commission on Human and Peoples' Rights, Advisory Opinion of the African Commission on Human and Peoples' Rights on the United Nations Declaration on the Rights of Indigenous Peoples, S. $30 \mathrm{f.}$

397 Siehe zu diesem Themenkomplex umfassend: Sub-Commission on Prevention of Discrimination and Protection of Minorities, ,Working Paper by the Chairperson - Rapporteur, Mrs. Erica-Irene A. Daes on the concept of, indigenous people“", UN Doc. E/CN.4/Sub.2/AC.4/1996/2, 10. Juni 1996.

398 Adelfo Regino Montes und Gustavo Torres Cisneros, „The United Nations Declaration on the Rights of Indigenous Peoples: the Foundation of a New Relationship between Indigenous Peoples, States and Societies“", in: Claire Charters und Rodolfo Stavenhagen (Hrsg.), Making the Declaration Work, the United Nations Declaration on the Rights of Indigenous Peoples, 2009, S. 152.

${ }^{399}$ John Henriksen, ,The UN Declaration on the Rights of Indigenous Peoples: Some Key Issues and Events in the Process", in: Claire Charters und Rodolfo Stavenhagen (Hrsg.), Making the Declaration Work, the United Nations Declaration on the Rights of Indigenous Peoples, 2009, S. $79 \mathrm{f}$.
} 
bar zu machen sind, aber erneut im modernen Völkerrecht an Bedeutung. In der Studie José Martínez Cobos, in den Instrumenten der ILO und der UN Deklaration und in einer Vielzahl weiterer Dokumente internationaler Organisationen wurde in intensiven Verhandlungen versucht, eine Definition zu finden. Neben dem formell anerkannten Ansatz in der ILO Convention No. 169, die mit nur 22 Ratifikationen vorrangig in Südarmerika Verbreitung gefunden hat, ist es vor allem die Arbeitsdefinition Cobos, die sich im internationalen Diskurs etabliert hat. Als ,indigene Völker` werden heute kulturell distinkte Gruppen mit einer historischen Verbindung zu präkolonialen Gesellschaften verstanden, die sich über eine gemeinsame Zusammengehörigkeit identifizieren und ihre Kultur zukünftigen Generationen weitergeben möchten.

Ein Teil der Begriffsbestimmung, indigener Völker' rekurriert auf eine traditionelle Lebensweise sowie auf eine Marginalisierung und Unterdrückung durch die Kolonisatoren und heute dominierende Mehrheitsgesellschaft. Im Sinne dieser Interpretation des Begriffs werden Ansprüche indigener Gruppen in jüngerer Zeit auch in Asien und Afrika artikuliert, wo das historische Differenzierungsmoment der Verbindung zu präkolonialen Gesellschaften keine tragfähigen Ergebnisse liefert. Ausgehend vom Kern der Selbstidentifikation von Gruppen als ,Indigene in Anlehnung an ihre Verbindung zu präkolonialen Gesellschaften, beziehungsweise ihre gegenwärtige Marginalisierung und Unterdrückung findet das Konzept der indigenen Völker heute weltweit Anwendung.

Das Fehlen einer Definition, indigener Völker ${ }^{6}$ in der UNDRIPs eröffnet einerseits Raum für eine flexible und globale Anwendung, bedarf aber gleichzeitig immer wieder der individuellen Durchsetzung der Anerkennung einer bestimmten Gruppe als indigen. So schließt auch die Sonderberichterstatterin der UN Working Group on Indigenous People Erica-Irene Daes zur Frage der Definition ihren Bericht mit dem Ausblick, dass die Implementierung der Deklaration über ein Organ erfolgen solle, ,which is fair-minded and open to the views of indigenous peoples and governments, so that there is room for the reasonable evolution and regional specificity of the concept of 'indigenous' in practice“ .400

\footnotetext{
400 Sub-Commission on Prevention of Discrimination and Protection of Minorities, ,W Working Paper by the Chairperson - Rapporteur, Mrs. Erica-Irene A. Daes on the concept of ,indigenous people“, UN Doc. E/CN.4/Sub.2/AC.4/1996/2, Abs. 74.
} 


\section{Lokale Gemeinschaften}

Im Umweltvölkerrecht findet sich eine Reihe von Bezeichnungen für kollektive Rechtsträger, denen aufgrund ihrer traditionellen Praxen umweltrechtliche Sonderregelungen eingeräumt werden. So wurden bereits im 19. Jahrhundert den damals sogenannten tribal peoples und indians besondere Rechte eingeräumt, die ihren traditionellen Handel mit Robbenpelzen regelten. Seit den 1980er Jahren entwickelten sich unter dem Dach der FAO die farmer's rights, wobei die Bedeutung der farmer für die Entwicklung und Bewahrung pflanzengenetischer Vielfalt in den Verhandlungen zur CBD aufgegriffen wurde. Und durch den Einfluss der internationalen Indigenenbewegung findet seit der Rio Konferenz 1992 das Begriffspaar der, indigenous and local communities im Umweltvölkerrecht des UN Systems neben dem grundlegenden Begriff der lokalen Gemeinschaft Verwendung. ${ }^{401}$ Die verschiedenen Instrumente treffen für diese Gruppen Regelungen zu ihrem traditionellen Wissen und ihren genetischen Ressourcen. ${ }^{402}$ Im Folgenden soll der Bedeutung des in der CBD geprägten Begriffspaars der ,indigenen und lokalen Gemeinschaften' nachgegangen werden, da diese Gemeinschaften im CBD-Regelungsrahmen insbesondere durch die Konkretisierung durch das Nagoya Protokoll eine eigene Rechtsposition genießen und deshalb als Rechtsträger identifizierbar sein müssen.

\section{Indigene und lokale Gemeinschaften in der CBD}

Die $C B D$ ist die bedeutendste Primärquelle, die das Begriffspaar der ,indigenen und lokalen Gemeinschaft ${ }^{\star}$ normiert. Sie enthält jedoch keine ausdrückliche Bestimmung, wie indigene und lokale Gemeinschaften definiert werden. Aus Artikel 8(j) CBD lässt sich allerdings die Zielrichtung der Regelungen zu diesen Gruppen und daraus indirekt ein Bedeutungsgehalt erschließen. Danach soll jede Vertragspartei, soweit möglich und angebracht:

Subject to its national legislation, respect, preserve and maintain knowledge, innovations and practices of indigenous and local communities embodying traditional lifestyles relevant for the conservation and sustainable use of biological diversity and promote their wider application with the approval and involvement of the holders of such knowledge, innovations and practices and encourage the equitable sharing of the benefits

\footnotetext{
401 Siehe dazu „C. I. Geschichte der indigenen und lokalen Gemeinschaften im Umweltvölkerrecht", auf S. 59.

402 Siehe dazu „C. III. Regelungen zum Schutz traditionellen Wissens“ auf S. 76.
} 
arising from the utilization of such knowledge, innovations and practices. ${ }^{403}$

Die CBD bezieht sich also auf Gruppen mit traditioneller Lebensweise, deren Wissen, Innovationen und Gebräuche eine entscheidende Rolle für die Bewahrung biologischer Vielfalt in-situ spielen. ${ }^{404}$ Auch in der Präambel der CBD wird indirekt der Bedeutungsgehalt des Begriffs, indigene und lokale Gemeinschaften' normiert:

Recognizing the close and traditional dependence of many indigenous and local communities embodying traditional lifestyles on biological resources, and the desirability of sharing equitably benefits arising from the use of traditional knowledge, innovations and practices relevant to the conservation of biological diversity and the sustainable use of its components. ${ }^{405}$

Hier wird deutlich, dass indigene und lokale Gemeinschaften als Gruppen verstanden werden, die Träger von traditionellem Wissen sind und damit einen Beitrag zur nachhaltigen Bewahrung biologischer Vielfalt leisten.

In der $C B D$ wurde also nicht nur der bereits geprägte Begriff der, indigenen Völker‘ oder ,indigenen Gemeinschaften' verwendet und dessen Bedeutungsgehalt übernommen. Man einigte sich vielmehr auf einen umfassenden Gruppenbegriff, der jegliche Gruppen traditioneller Lebensweise umfasst, die zur Bewahrung biologischer Vielfalt beitragen. „This phrase is interpreted to include the estimated 1.5 to 2 billion people around the world who have not adopted industrialized practices to exploit agricultural, forest, animal and fisheries resources. "406 Es geht also neben indigenen Völkern auch um Bauern, Fischer, ländliche Gemeinschaften und Nomadengruppen, die einer traditionellen, nicht industriellen Lebensweise folgen. 407

II. Unterschiede zwischen indigenous peoples, indigenous communities und local communities

Die Bezeichnung ,lokale Gemeinschaft' wird im Kontext des Umweltrechts nicht nur als gleichbedeutender Gegenvorschlag zum Begriff der ,indigenen Völker'verwendet. Zwar dient er als Alternative, ,um so die Schwierigkeiten der

\footnotetext{
403 Convention on Biological Diversity, Art. 8(j).

404 Posey, „Identifizierung und Respektierung der Grenzen zwischen indigenen Völkern, traditionellen Bauern und örtlichen Gemeinschaften", S. 242.

405 Convention on Biological Diversity, Abs. 12.

406 Office of the High Commissioner for Human Rights, „United Nations Guide for Indigenous Peoples, Leaflet No. 10: Indigenous Peoples and the Environment", 2001, S. 6.

407 Posey, „Identifizierung und Respektierung der Grenzen zwischen indigenen Völkern, traditionellen Bauern und örtlichen Gemeinschaften", S. 246.
} 
genauen Definition von indigenen Gruppen zu vermeiden." "408 Die beiden Begriffe unterscheiden sich jedoch insbesondere bezüglich der ihnen implizierten Rechte und Ansprüche, den beschriebenen Praxen und Lebensweisen sowie in der geographischen Verbreitung ihrer Anwendung von Seiten der Staaten.

\section{Implizierte Rechte und Ansprïche}

Ein grundlegender Unterschied zwischen den beiden Begriffen der ,indigenen Völker' und der ,lokalen Gemeinschaften' ergibt sich aus den verschiedenen Regelungsumfängen, die historisch bedingt mit diesen Begriffen verknüpft sind. So ist die Bezeichnung people seit jeher problematisch, da sie als völkerrechtlich geprägter Begriff den Anspruch auf das Selbstbestimmungsrecht der Völker im Sinne von Artikel $1 \mathrm{IPbpR}$ vorgibt. Dieser Inhalt wird durch die Verbindung mit der Bezeichnung indigenous noch verstärkt, da es das Argument der zeitlich vorausgehenden Besiedelung des Landes explizit in der Bezeichnung verankert. So sind indigenous people die ursprünglichen Bewohner ihres Landes von dem sie im Zuge der Kolonisierung gewaltsam vertrieben wurden. Mit der gewaltsamen und unrechtmäßigen Vertreibung von ihrem Land begründen sie nun den umfassenden Anspruch auf Selbstbestimmung im völkerrechtlichen Sinne und erheben Anspruch auf ihr Land. Auch wenn das Recht auf Selbstbestimmung indigener Völker heute im menschenrechtlichen Kontext nicht mehr als Recht auf Sezession interpretiert wird, konstatiert es den Anspruch der Gruppe, selbst über ihre politischen, wirtschaftlichen, sozialen und kulturellen Belange entscheiden zu können. Diese Selbstbestimmungsrechte schließen zwar die Regelungsmaterie des Umweltvölkerrechts ein, gehen aber auch weit darüber hinaus.

Die Rechte und Regelungen, die im internationalen Umweltrecht für indigene und lokale Gemeinschaften getroffen werden, knüpfen an die nachhaltige Lebensweise dieser Gruppen an und beziehen sich auf ihre Rolle zum Schutz und zur Förderung biologischer und pflanzengentischer Vielfalt. Sie regeln die Einbeziehung und Entscheidungsbeteiligung dieser Gruppen, ihre Zustimmung und Vorteilsteilhabe beim bioprospecting, sowie die Rechte an ihrem traditionellen Wissen im Kontext genetischer Ressourcen. ${ }^{409}$

Der Anspruch auf Selbstbestimmung der politischen Bewegung indigener Völker reicht somit weit über die Beteiligungs- und Zustimmungsrechte hinaus, die für lokale Gemeinschaften im Kontext des internationalen Umweltrechts normiert werden. Zwar wäre rechtstechnisch eine differenzierte Behandlung der Ansprüche indigener Völker auf Selbstbestimmung auf der einen Seite und der hier gewährten Rechte auf der anderen möglich, da der Rechtsumfang im Grunde

\footnotetext{
408 von Hahn, Traditionelles Wissen indigener und lokaler Gemeinschaften zwischen geistigen Eigentumsrecbten und der ,public domain", S. 31.

${ }^{409}$ Siehe dazu „C. III. Regelungen zum Schutz traditionellen Wissens“ auf S. 76.
} 
keine Aussage über die Definition des Rechtsträgers trifft. Durch die Verquickung der politischen Implikationen des umfassenden Anspruchs auf Selbstbestimmung indigener Völker wurde für die Regelungen im Umweltrecht jedoch ein neuer, weniger politisch aufgeladener Begriff gewählt.

\section{Vergleichbare Praxen und Lebensweisen}

Auch wenn viele indigene Völker örtliche Bauern sind und subsistenzwirtschaftlich Leben sowie indigene und lokale Gemeinschaften im Verhältnis zur dominierenden Bevölkerung in vergleichbaren Situationen sind, geht das moderne Verständnis, was indigene Völker ausmacht, über das Bild einer lokalen Gemeinschaft hinaus. ${ }^{410}$ Indigene zeichnen sich grundsätzlich durch ihre besondere Beziehung zu ihrem Land und ihrer Umwelt in Form einer besonderen spirituellen Bindung aus.

Das romantisierte Bild indigener Völker wird heute jedoch zunehmend aufgebrochen und diversifiziert. Die Bedeutung der Bezeichnung ,indigenes Volk' bezieht sich in einem modernen Verständnis nicht mehr nur auf das Bild des, edlen Wilden' der als Einheit mit seiner Umwelt in subsistenzwirtschaftlicher Weise von den Früchten der Natur lebt. Indigenität bedeutet heute mehr als solch ein reduzierendes Bild, welches durch Geschichten, Filme und die Medien konstruiert wird. So leben indigene Völker heute auch in Städten, haben ,normale` Arbeitsplätze, betreiben Kasinos und fördern Öl auf ihrem Land. Die Bezeichnung, indigene Völker' wäre in diesem modernen Verständnis zu weit, um im Kontext umweltrechtlicher Regelungen die Gruppen der Rechtsträger zu beschreiben. Artikel 8(j) CBD trifft dahingehend die qualifizierende Einschränkung, welche die Anwendbarkeit auf Gruppen „embodying traditional lifestyles relevant for the conservation and sustainable use of biological diversity" beschränkt.

\section{Kompromiss geographischer Anwendbarkeit}

Das Begriffspaar der ,indigenous and local communities` entwickelte sich auch aus der bereits dargestellten geographisch differenzierten Anwendbarkeit des Begriffs ,indigene Völker. ${ }^{6} 11$ So widersprechen asiatische und afrikanische Staaten grundsätzlich der Anwendbarkeit des Konzepts ,Indigenität ${ }^{`}$ in ihren Ländern. ${ }^{412}$ Um umweltrechtlichen Regelungen dennoch zu globaler Anwendbarkeit zu verhelfen, einigte man sich für die $C B D$ auf die Kompromissformel der ,indigenen

\footnotetext{
${ }^{410}$ Posey, „Identifizierung und Respektierung der Grenzen zwischen indigenen Völkern, traditionellen Bauern und örtlichen Gemeinschaften", S. 247; von Hahn, Traditionelles Wissen indigener und lokaler Gemeinschaften zwischen geistigen Eigentumsrechten und der „public domain“, S. 32; Kingsbury, „,Indigenous Peoples“ in International Law“, S. 451.

411 Siehe dazu „B. IV. Regionale Interpretationen in Asien und Afrika“ auf S. 98.

412 Ebd.
} 
und lokalen Gemeinschaften` beziehungsweise auf den neuen umweltvölkerrechtlichen Ausdruck der , lokalen Gemeinschaft ${ }^{\star}{ }^{413}$

Zwar zeigen aktuelle Entwicklungen, dass lokale Bauern auch in solchen Regionen der Welt, die nicht durch typische Siedlergesellschaften geprägt sind, ihre territoriale Verwurzelung und kulturelle Besonderheit nutzen, um in einer politischen Selbstidentifikation Ansprüche als ,Indigene' geltend zu machen. ${ }^{414}$ Das Argument der mangelnden globalen Anwendbarkeit wird somit zunehmend überwunden. Doch trotz dieser Vermischung unterschiedlicher lokaler politischer Initiativen mit dem globalen Indigenitätsdiskurs hat der Ausdruck , lokale Gemeinschaft ${ }^{`}$ in seiner inhaltlichen Konkretisierung im Umweltrecht eine eigenständige Bedeutung erlangt. Gerade in den Staten, in denen die Existenz von ,indigenen Völkern' von Staatsseite verneint wird, können Gruppen unter dem Begriff der ,lokalen Gemeinschaft' umweltrechtliche Ansprüche geltend machen. ${ }^{415}$

\section{Schlussfolgerungen}

Untersucht man das Begriffspaar der, indigenous and local communities' in Hinblick auf seine Bedeutung des völkerrechtlich neueren Begriffs der ,lokalen Gemeinschaft' in Abgrenzung zum etablierten Begriff der, indigenen Völker', ergibt sich eine grundlegende Unterscheidung nach dem implizierten Rechtsumfang. Der Anspruch auf Selbstbestimmung, welcher der Selbstidentifikation als, indigenes Volk' inhärent ist, wird von dem Rechtskonzept der ,indigenen und lokalen Gemeinschaft ${ }^{`}$ nicht umfasst. ${ }^{416}$ Das Konzept der , lokalen Gemeinschaft ${ }^{`}$ kann in Bezug auf den Rechtsumfang mit der normativen Kraft der Indigenenbewegung nicht mithalten. ${ }^{417}$ Vielmehr bildet es eine Alternative zur politisch aufgeladenen Bewegung indigener Völker und genießt somit eine umfassendere $\mathrm{Zu}$ stimmung von staatlicher Seite.

III. Das ad hoc expert meeting of local-community representatives

Eine genaue Definition oder konkrete Bedeutung des Begriffs ,lokale Gemeinschaften' konnte bisher noch nicht festgeschrieben werden. In Anbetracht der im Verhältnis zur Beteiligung von Vertretern indigener Gruppen sehr ungenügen-

\footnotetext{
413 von Hahn, Traditionelles Wissen indigener und lokaler Gemeinschaften zwischen geistigen Eigentumsrechten und der ,public domain", S. 33.

${ }^{414}$ Klenke und Socha, „Emerging Indigeneity - Völkerrechtswissenschaft und ethnologische Praxis subnationaler kultureller Gemeinschaften".

415 von Hahn, Traditionelles Wissen indigener und lokaler Gemeinschaften zwischen geistigen Eigentumsrechten und der ,public domain", S. 33.

416 Posey, „Identifizierung und Respektierung der Grenzen zwischen indigenen Völkern, traditionellen Bauern und örtlichen Gemeinschaften“, S. 256.

417 Kingsbury, ,„Indigenous Peoples“ in International Law“, S. 453; von Hahn, Traditionelles Wissen indigener und lokaler Gemeinschaften zwischen geistigen Eigentumsrechten und der „public domain“, S. 33.
} 
den Repräsentation von lokalen Gemeinschaften setzte die Conference of the Parties 10 der CBD (COP 10) am 29. Oktober 2010 im Rahmen des langjährigen Arbeitsprogramms zur Umsetzung von Artikel 8(j) CBD ein ad hoc expert group meeting of local-community representatives ein. Das Treffen diente der Klärung des Bedeutungsgehalts des Konzepts ,lokale Gemeinschaft', sollte Vorschläge sammeln wie diese Gruppen besser in die Verhandlungen integriert werden können und wie die Umsetzung der $C B D$ für diese Gruppen unterstützt werden könnte. ${ }^{418}$ Den 34 Experten wurde zu ihrem Treffen im Juli 2011 von Seiten des Sekretariats ein Leitfaden zum Vorgehen ${ }^{419}$, ein Bericht zur Teilnahme von lokalen und indigenen Gemeinschaften ${ }^{420}$ sowie ein Informationsdokument zum Konzept ,lokale Gemeinschaften' des UNPFII ${ }^{421}$ zur Verfügung gestellt. In ihrem Bericht an die Working Group on Article 8(j) gaben sie folgende Anhaltspunkte, die neben der essentiellen Selbstidentifikation als local community, für eine Arbeitsdefinition berücksichtigt werden sollten:

Local communities living in rural and urban areas of various ecosystems may exhibit some of the following characteristics:

(a) Self-identification as a local community;

(b) Lifestyles linked to traditions associated with natural cycles (symbiotic relationships or dependence), the use of and dependence on biological resources and linked to the sustainable use of nature and biodiversity;

(c) The community occupies a definable territory traditionally occupied and/or used, permanently or periodically. These territories are important for the maintenance of social, cultural, and economic aspects of the community;

(d) Traditions (often referring to common history, culture, language, rituals, symbols and customs) and are dynamic and may evolve;

418 Conference of the Parties to the Convention on Biological Diversity, „Decision adopted by the Conference of the Parties to the Convention on Biological Diversity at its Tenth Meeting, X/43. Multiyear programme of work on the implementation of Article $8(\mathrm{j})$ and related provisions of the Convention on Biological Diversity“, UNEP/CBD/COP/DEC/X/43, 29. Oktober 2010, Abs. 21.

${ }^{419}$ Expert Group Meeting of Local Community Representatives within the Context of Article 8(j) and Related Provisions of the Convention on Biological Diversity, „Guidance for the Discussions Concerning Local Communities within the Context of the Convention on Biological Diversity“, UNEP/CBD/AHEG/LCR/2, 7. Juli 2011.

420 Ad hoc open ended working group on Article 8(j) and Related Provisions of the Convention on Biological Diversity, ,Participatory Mechanisms for Indigenous and Local Communities in the Work of the Convention“, UNEP/CBD/WG8J/7/9, 8. Juli 2011.

421 Ad hoc Expert Group Meeting of Local Community Representatives, ,Identification of Common Characteristics of Local Communities“, UNEP/CBD/AHEG/LCR/INF/1, 27. Juni 2011. 
(e) Technology/knowledge/innovations/practices associated with the sustainable use and conservation of biological resources;

(f) Social cohesion and willingness to be represented as a local community;

(g) Traditional knowledge transmitted from generation to generation including in oral form ${ }^{422}$

Des Weiteren werden folgende Anhaltspunkte aufgezählt: gemeinsame soziale Normen, Gewohnheitsrecht, Gruppenrechte, Selbstregulierung durch Tradition und traditionelle Organisationsformen und Institutionen, Subsistenzwirtschaft und nachhaltige Entwicklung, biologisches und kulturelles Erbe, spirituelle und kulturelle Werte bezüglich Biodiversität und Land, Kultur und traditionelle kulturelle Ausdrucksformen, die von einer lokalen Sprache umfasst werden. Ebenfalls genannt werden Nahrung, Nahrungszubereitung und traditionelle Medizin, die in die Biodiversität und Umwelt eingebunden sind sowie eine Marginalisierung von modernen geopolitischen Systemen und Strukturen. Weiterhin heißt es:

(r) May have had little or no prior contact with other sectors of society resulting in distinctness or may choose to remain distinct;

(s) Practice of traditional occupations and livelihoods;

(t) May live in extended family, clan or tribal structures;

(u) Belief and value systems, including spirituality, are often linked to biodiversity;

(v) Shared common property over land and natural resources;

(w) Traditional right holders to natural resources;

(x) Vulnerability to outsiders and little concept of intellectual property rights. ${ }^{423}$

Das hier gezeichnete Bild lokaler Gemeinschaften entspricht wohl am ehesten dem der Völker, die bisher noch wenig oder keinen Kontakt zu ihrer Außenwelt haben, wie sie im Regenwald Brasiliens und Perus leben. ${ }^{424}$ Dies würde den Anwendungsbereich des Konzepts erheblich einschränken. Jedoch sollen diese Anhaltspunkte nur Anstoß für weitere Konkretisierung sein und bilden keine kumulativ notwendigen Voraussetzungen einer rechtlichen Definition.

\footnotetext{
422 Ad hoc open ended working group on Article 8(j) and Related Provisions of the Convention on Biological Diversity, „Report of the Expert Group Meeting of Local Community Representatives within the Context of Article 8(j) and Related Provisions of the Convention on Biological Diversity“, UNEP/CBD/WG8J/7/8/ADD1, 4. September 2011, Annex. ${ }^{423}$ Ebd.

${ }^{424}$ Vgl. die Darstellung unter: Survival International, „Unkontaktierte Völker Brasiliens“, zugegriffen 3. Januar 2017, http://www.survivalinternational.de/indigene/unkontaktiertebrasilien.
} 
In der Sitzung der Arbeitsgruppe zu Artikel 8(j) CBD wurde über das Dokument des ad hoc expert meeting of local-community representatives im Rahmen der Diskussionen zur Beteiligung von indigenen und lokalen Gemeinschaften beraten. ${ }^{425}$ Die Arbeitsgruppe machte der COP 11 den Vorschlag, den Mitgliedstaaten der CBD zu empfehlen, den Bericht des expert meetings als mögliche Grundlage zur Bestimmung lokaler Gemeinschaften zur Kenntnis zu nehmen sowie den 13. Juli als ,internationalen Tag der lokalen Gemeinschaften`auszurufen. ${ }^{426}$ Eine weiterführende Konkretisierung einer juristischen Definition steht somit noch aus.

\section{Geographische Verbreitung lokaler Gemeinschaften}

Hervorzuheben ist neben dem Konsens zur Schlüsselrolle der Selbstidentifikation, dass das Konzept der lokalen Gemeinschaft überall auf der Welt Anwendung finden könnte. So führte das Sekretariat in ihrem Leitfaden für das Expertentreffen aus, dass

[m]any communities may be considered local and may also be described as traditional communities. Some local communities may include peoples of indigenous descent. They are culturally diverse and occur on all inhabited continents. For example, small farming communities in France, who have occupied and farmed their lands for many generations acquiring useful environmental knowledge including specialist knowledge about a variety of activities including sustainable agriculture, cheese making and wine making or even animal husbandry may represent a local or traditional community. Long term established rice and fish farmers in Asia may represent another type of local community. In Brazil, local and traditional communities are an important segment of the national population and are a legal entity in the national constitution. ${ }^{427}$

Die hier genannten Beispiele der kleinen Agrargemeinschaften in Frankreich, den Reis- und Fischfarmen in Asien oder lokalen und traditionellen Gemeinschaften in Brasilien verdeutlichen, dass das Konzept der lokalen Gemeinschaft globale Anwendung finden soll. Die Verbreitung von ,lokalen Gemeinschaften“

${ }^{425}$ Conference of the Parties to the Convention on Biodiversity, ,,Report of the Seventh Meeting of the Ad Hoc Open-Ended Inter Sessional Working Group on Article 8(j) and Related Provisions of the Convention on Biological Diversity“, UNEP/CBD/COP/11/7, 24. November 2011, ITEM 4, Abs. 33-44.

${ }^{426}$ Ebd., Annex I, Recommendation 7/2, Abs. 17-21.

${ }^{427}$ Expert Group Meeting of Local Community Representatives within the Context of Article 8(j) and Related Provisions of the Convention on Biological Diversity, „Guidance for the Discussions Concerning Local Communities within the Context of the Convention on Biological Diversity“, UNEP/CBD/AHEG/LCR/2, Abs. 5. 
bestimmt sich dabei im Grunde nach dem Vorkommen von Biodiversität, die grundsätzlich in äquatornahen Gebieten größer ist, aber auch Schwerpunkte in Zentraleuropa aufweist. ${ }^{428}$ Der Begriff der ,lokalen Gemeinschaft ${ }^{`}$ wird insbesondere von den afrikanischen Staaten befürwortet, die sonst die Anwendbarkeit von Konzepten substaatlicher Gruppen in ihren Ländern eher ablehnen. ${ }^{429}$ Allerdings äußerten sich die Staaten Nordamerikas in ablehnender Haltung gegenüber der Anwendbarkeit des Konzepts ,lokaler Gemeinschaften‘. Im Composite Report on the status and trends regarding the knowledge, innovations and practices of indigenous and local communities für Nordamerika findet sich der Hinweis, dass hier das Konzept der lokalen Gemeinschaft mangels einer konkreten Definition bisher keine Anwendung finde. ${ }^{430}$ Jedoch wird hier mit dem bereits geprägten Konzept der, indigenen Völker ${ }^{6}$ gearbeitet. Es bestätigt sich somit die ursprüngliche Intention, das Konzept local community als bloße Alternative zum Begriff der ,indigenen Völker' zu etablieren, indem es sich vorrangig dort durchsetzt, wo das Konzept der Indigenität abgelehnt wird.

\section{Lokale Gemeinschaften und Farmer's Rights}

Das Umweltrecht verknüpft Initiativen der globalen Indigenenbewegung und Entwicklungen im Bereich der Landwirtschaft. So wurde beispielsweise bereits in den 1980er Jahren die Rolle von Bauern bezüglich pflanzengenetischer Vielfalt unter dem Dach der FAO normiert, was schließlich auch in den Verhandlungen zur $C B D$ Beachtung fand. Sie spielten somit neben den in diesem Bereich grundsätzlich gut vertretenen indigenen Völkern eine entscheidende Rolle. Mitte der 1990er Jahren kam es zu einer weiteren Verknüpfung der Politiken und Regelungsbereiche indigener Völker und bäuerlicher Gemeinschaften. So waren es zunächst Vertreter bäuerlicher Gemeinschaften, die sich in ihren Forderungen auf das Selbstbestimmungsrecht beriefen und so die Rhetorik der Indigenenbewegung für sich politisch nutzbar machten. Im Gegenzug dehnten Vertreter indigener Gruppen ihre Aktivitäten und Forderungen in den Bereichen der Landwirtschaft, der Landrechte und des pflanzengenetischen Materials aus. $^{431}$

\footnotetext{
${ }^{428}$ Susette Biber-Klemm und Danuta Szymura Berglas, „General Framework, Problems and Goals“, in: Susette Biber-Klemm und Thomas Cottier (Hrsg.), Rights to plant genetic resources and traditional knowledge: basic issues and perspectives, 2006, S. 15.

${ }^{429}$ von Hahn, Traditionelles Wissen indigener und lokaler Gemeinschaften zwischen geistigen Eigentumsrechten und der ,public domain", S. 33.

430 Ad hoc open ended working group on Article 8(j) and Related Provisions of the Convention on Biological Diversity, „Composite report on the status and trends regarding the knowledge, innovations and practices of indigenous and local communities - Regional report: North America“, UNEP/CBD/WG8J/3/INF/8, 7. Oktober 2003, S. 4.

431 Posey, „Identifizierung und Respektierung der Grenzen zwischen indigenen Völkern, traditionellen Bauern und örtlichen Gemeinschaften", S. 248.
} 
In den vergleichbaren Regelungen des Artikel 8(j) CBD und Artikel 9 des Internationalen Abkommens zu Pflanzengenetischen Ressourcen für Nabrung und Landwirtschaft (ITPGRFA) von 2001, der auch von „local and indigenous communities and farmers" spricht, wird die Nähe dieser beiden Regelungsbereiche deutlich. Beide beziehen sich auf Gruppen, die durch ihre traditionellen Praxen einen Beitrag zu Erhalt und Förderung biologischer bzw. pflanzengenetischer Vielfalt leisten und treffen Regelungen zu traditionellem Wissen in Bezug auf genetische Ressourcen von Nahrung und Landwirtschaft, Zugang und gerechter Vorteilsteilhabe sowie zu Beteiligungsrechten in Entscheidungsverfahren zum Schutz und zur nachhaltigen Nutzung von pflanzengenetischen Ressourcen.

So entwickelte das Sekretariat der CBD in Zusammenarbeit mit der FAO eine Studie zu den Auswirkungen des ITPGRF $A$ auf den Bedeutungsgehalt der Regelungen des Artikel 8(j) CBD, in der es feststellt, dass

[n]either of the terms ,indigenous and local communities embodying traditional lifestyles' or ,farmers' is defined, in the Convention on Biological Diversity or the [ITPGRFA] Treaty, respectively. Both groups, whether separately, or together, constitute a significant proportion of the world's population and bear significant responsibility for global food and medicinal security based on their development, conservation and use of plant genetic resources. It would also seem apparent that there must in practice be a large element of overlap between the two groups. $[\ldots]$

Both on-farm and wild crops relatives in situ are of course essential for sustainable agriculture, and may in practice be the concern of the same or overlapping groups. To this extent, Article 8(j) of the Convention on Biological Diversity and Article 9 of the Treaty can be seen as mutually reinforcing, although not necessarily covering the same ground or at least not from the same precise perspective. ${ }^{432}$

,Bauern' bilden somit wohl eine große, aber nicht die einzige Gruppe im Sinne der ,lokalen Gemeinschaften mit traditioneller Lebensweise‘ des Artikel 8(j) CBD. ${ }^{433}$ Der Begriff bezieht sich dabei nicht nur auf Bauern die Getreide anbauen, sondern schließt auch Viehwirtschaft oder ,Jagen, Fischen und die Nutzung halbdomestizierter Arten von Bäumen und Nichtholzpflanzen als Brenn-

432 Conference of the Parties to the Convention on Biological Diversity, ,The implications of the international treaty on plant genetic resources for food and agriculture on the issues under Article 8(j) and related provisions“, UNEP/CBD/COP/7/INF/18, 17. Dezember 2003, Abs. 19-20.

433 Posey, „Identifizierung und Respektierung der Grenzen zwischen indigenen Völkern, traditionellen Bauern und örtlichen Gemeinschaften“, S. 247. 
stoff, Nahrungsmittel, Arzneimittel oder anderen häuslichen Gebrauch“ mit ein. ${ }^{334}$

\section{Zusammenfassung}

Das Konzept der , lokalen Gemeinschaft' mit traditioneller Lebensweise wie es sich im Kontext des Umweltvölkerrechts und insbesondere unter dem Begriffspaar der ,indigenen und lokalen Gemeinschaft ${ }^{`}$ in $\operatorname{der} C B D$ entwickelt hat, umfasst Gruppen, die in unmittelbarer Abhängigkeit von ihrer Umwelt leben und in subsistenzwirtschaftlicher Lebensweise einen Beitrag zu Erhalt und Förderung biologischer Vielfalt leisten. Dieses Konzept begreift Gruppen in ihren Aktivitäten wie sie den übergeordneten Belangen des Umweltrechts gerecht werden und normiert damit den Regelungskontext ,Umweltschutz' als Voraussetzung für das Verständnis, was eine ,lokale Gemeinschaft' ausmacht.

Die beiden Teile des CBD Begriffspaares, indigene und lokale Gemeinschaften" unterscheiden sich vor dem historischen Hintergrund der Indigenenbewegung im implizierten Anspruch auf Selbstbestimmung. Diese Unterscheidung anhand des Rechtsumfangs trifft jedoch keine Aussage über die Konzeption des Rechtsträgers. Dafür ergibt sich aus der Verwendung als Begriffspaar, dass die dem Konzept der ,lokalen Gemeinschaft ${ }^{\natural}$ zugrunde liegende Idee an das Konzept indigenes Volk' angelehnt ist. Es kommt also auch hier auf eine kulturelle Distinktion und entsprechende Selbstidentifikation an. Durch den Regelungskontext ergibt sich zusätzlich die Einschränkung auf Gruppen traditioneller Lebensweise, die sich durch die nachhaltige Bewahrung von Biodiversität auszeichnen.

,Lokale Gemeinschaften' werden somit im Wesentlichen über ihre Wirtschaftsform definiert, ${ }^{435}$ die sich in einer traditionellen, subsistenzwirtschaftlichen Lebensweise konkretisiert, der inhärente Nachhaltigkeit und Biodiversität begünstigende Eigenschaften zugeschrieben werden. Diese Interpretation ergibt sich allerdings nicht aus der Übertragung des romantisierten Bildes des ,edlen Wilden indigener Völker, sondern aus der Konkretisierung, dass sich das Umweltvölkerrecht nur auf Gruppen mit ,traditioneller Lebensweise' bezieht. Auch wenn viele Gruppen, die sich als indigene Völker identifizieren, örtliche Bauern sind und nachhaltige Subsistenzwirtschaft betreiben, hat die Verwendung der Bezeichnung ,indigen' im Kontext der Regelungen zu biologischer Vielfalt keine Rückwirkungen einer Uminterpretation des Indigenitätskonzepts. Es verbietet sich der Rückschluss, dass sich aufgrund der Bedeutung indigener Gruppen im Umweltvölkerrecht nun eine Konkretisierung entwickelt, nach der alle Indigenen zwingend einer

\footnotetext{
434 Ebd.

435 von Hahn, Traditionelles Wissen indigener und lokaler Gemeinschaften zwischen geistigen Eigentumsrecbten und der ,public domain", S. 32.
} 
nachhaltigen Lebensweise folgen müssten. ${ }^{436}$ Die konkretisierende Einschränkung auf Gruppen mit einer nachhaltigen Subsistenzwirtschaft ergibt sich im Umweltvölkerrecht vielmehr aus der Zielrichtung und dem Regelungsinteresse Biodiversität zu bewahren und zu fördern. Aus der Verbindung der überlagernden Interessen lokaler Bauern, Jäger und Fischer auf der einen Seite und indigener Völker auf der anderen fand der Begriff der, indigenen und lokalen Gemeinschaft ${ }^{6}$ in der $C B D$ als eigenständiger Gruppenrechtsbegriff im Umweltvölkerrecht Verwendung, der Gruppen beschreibt, die sich in Selbstidentifikation durch eine nachhaltige subsistenzwirtschaftliche Lebensweise auszeichnen. Die inhaltliche Trennung wird zudem in dem eigenständigen Begriff ,lokale Gemeinschaft ${ }^{\star}$ deutlich, der so zum Beispiel in der Agenda 21 und dem Abschlussdokument von Rio+20 im Jahr 2012 getrennt vom Begriff ,indigene Völker` verwendet wurde.

436 Posey, „Identifizierung und Respektierung der Grenzen zwischen indigenen Völkern, traditionellen Bauern und örtlichen Gemeinschaften“, S. 247, $256 f$. 


\section{Selbstidentifikation kultureller Distinktion als Kern der Definitionsansätze}

Die Darstellung der drei völkerrechtlichen Konzepte substaatlicher Gruppen in Teil 1 sowie der unterschiedlichen Definitionsansätze in Teil 2 haben gezeigt, dass alle drei Konzepte durch ihre jeweilige Geschichte und die unterschiedlichen Reglungskontexte eine Prägung und Konkretisierung erfahren haben, welche die Begriffe ,Minderheit', ,indigenes Volk' und ,lokale Gemeinschaft' jeweils individualisieren und somit unterscheidbar machen. So lassen sich die typischen Idealbilder in ihren jeweiligen Kontexten im Kern folgendermaßen beschreiben: Minderheiten zeichnen sich durch ihre Minderzahl im Verhältnis zur Mehrheitsgesellschaft im Staat aus. Indigene Völker weisen eine Verbindung zu präkolonialen Gesellschaften auf oder erleiden gegenwärtig eine Marginalisierung oder Unterdrückung durch eine dominierende ,moderne' Gesellschaft. Lokale Gemeinschaften folgen einer nachhaltigen, biodiversitätsfördernden, subsistenzwirtschaftlichen Lebensweise.

Das Moment der Distinktion substaatlicher Gruppen gegenüber der Mehrheit wird dazu an unterschiedlichen Kriterien, wie etwa der unterschiedlichen Sprache, einer anderen Religion oder eigenen Spiritualität, an einer typischen Kleidung, an besonderen Ritualen oder einer speziellen wirtschaftlichen Tätigkeit festgemacht, die allgemein als eine ,andere Kultur ${ }^{6}$ beschrieben werden, deren Wurzeln sich zudem jeweils historisch belegen lassen. Die dargestellten Definitionsansätze nennen diese und weitere Aspekte als Anhaltspunkte, nach denen eine Minderheit, ein indigenes Volk oder eine lokale Gemeinschaft zu bestimmen sei. Alle Definitionen nutzen somit ein Merkmal einer kulturellen Distinktion der Gruppe, um ein Kollektiv von der Gruppe der Mehrheit unterscheidbar zu machen.

Vor allem rekurrieren alle Konzepte allerdings auf einen Ausdruck der Zusammengehörigkeit oder eine Selbstidentifikation der Gruppe. Die Gruppe selbst bringt ihre Zusammengehörigkeit zum Ausdruck und positioniert sich in ihrem Selbstverständnis in einer Rolle im Sinne eines der Konzepte. Im Bereich des Minderheitenschutzes wird dies als „Ausdruck des Gefühls der Verbundenheit der Gruppe“ oder „,sense of solidarity“ beschrieben. Im Kontext der Indigenenbewegung steht die Selbstidentifikation am Anfang jeder Überlegung zur Bestimmung indigener Völker. Und auch die Diskussionen zur Definition lokaler Gemeinschaften wie sie beispielsweise im ad hoc expert meeting of local-community experts geführt wurden, messen der self-identification as a local community entscheidende Bedeutung bei.

Wenn man nun die historisch und politisch geprägten, konkretisierenden Merkmale der Definitionsansätze ausblendet, wird deutlich, dass alle Ansätze auf einen gleichen Kern einer Selbstidentifikation der Gruppe rekurrieren, die sich an 
einer kulturellen Distinktion festmacht. Ian Brownlie formulierte diese Feststellung in der These, dass

the heterogeneous terminology which has been used over the years - the references to nationalities, peoples, minorities and indigenous peoples - involves essentially the same idea. ${ }^{437}$

Die völkerrechtlichen Definitionen folgen somit alle demselben Ansatz - ,derselben Idee' Ian Brownlies -, um eine Gruppe zu beschreiben. Dieser gemeinsame Ansatz ist die ,Selbstidentifikation kultureller Distinktion' einer Gruppe, die sich dann jeweils auf eines der völkerrechtlichen Konzepte bezieht.

${ }^{437}$ Brownlie, ,The Rights of Peoples in Modern International Law“, S. 5. 


\section{E. Das rechtliche Verhältnis der Konzepte zueinander}

In keinem der drei Bereiche des Völkerrechts, in dem sich Regelungen für substaatliche Gruppen finden - dem menschenrechtlichen Minderheitenschutz, den Entwicklungen zu Rechten indigener Völker, den Regeln für lokale Gemeinschaften im internationalen Umweltrecht - sind bisher allgemein anerkannte, abstrakt-generelle Definition der Konzepte festgeschrieben worden. Allerdings beziehen sich alle Definitionsansätze der Konzepte in ihrem Kern auf - wie Ian Brownlie schreibt - dieselbe Idee einer kollektiven kulturellen Identität, die durch die Selbstidentifikation der Gruppe zum Ausdruck gebracht wird. Gleichzeitig sind die drei Konzepte durch ihre jeweilige Geschichte, den jeweiligen Regelungskontext und auch in Hinblick auf die mit ihnen verknüpften Rechte, die einschlägigen Durchsetzungs- beziehungsweise Überwachungs- und Berichtsmechanismen grundsätzlich unterschiedlich.

Die Darstellungen in Teil 1 zeigten, dass der Minderheitenschutz mit seinen Grundlagen im Westfälischen Frieden von 1648 als ältestes Konzept für substaatliche Gruppen verschiedene Phasen individual- und kollektivrechtlicher Auslegung erfahren und sich ausgehend vom Religionsschutz bis heute zu einem starken Instrument für den umfassenden Schutz von Kultur entwickelt hat. ${ }^{438}$ Die Rechte indigener Völker, wie sie insbesondere in der Deklaration von 2007 Ausdruck finden, sind das Ergebnis eines jahrzehntelangen politischen Kampfes der globalen Indigenenbewegung, die sich vor dem Hintergrund der Kolonialgeschichte im Rahmen der Vereinten Nationen bis heute eine feste Position neben den Staaten als eigentlichen Akteuren im Völkerrecht erarbeitet haben. ${ }^{439}$ Lokale Gemeinschaften stehen im modernen Umweltvölkerrecht als Garanten für eine biodiversitätsfördernde, subsistenzwirtschaftliche Lebensweise, die in ihrem jeweiligen lokalen Kontext über die Regelungen des prior informed consent gegenüber multinationalen Konzernen im globalen Nord-Süd-Konflikt eine eigene Rolle zugewiesen bekommen haben. ${ }^{440}$ Trotz des gemeinsamen Kerns der bisher diskutierten Definitionsansätze entsteht angesichts ihrer Unterschiedlichkeit der Eindruck, die drei Konzepte stünden in einem Verhältnis sich ausschließender Exklusivität zueinander.

Allerdings hat die vorangegangene Darstellung viele Verknüpfungen und überlagernde Anwendbarkeiten der Konzepte aufgezeigt: Indigene Völker werden beispielsweise seit jeher im Kontext des Minderheitenschutzes verhandelt; im

\footnotetext{
${ }^{438}$ Siehe dazu „A. V. Zusammenfassung: ältestes Konzept zum Schutz kulturell distinkter Gruppen“ auf S. 36.

${ }^{439}$ Siehe dazu „B. V. Zusammenfassung: globale politische Bewegung für Selbstbestimmung“ auf S. 58.

${ }^{440}$ Siehe dazu „C. V. Zusammenfassung: neue Akteure beim Schutz von Biodiversität“ auf S. 79.
} 
internationalen Umweltrecht spielen indigene Völker eine wichtige Rolle, woraus sich insbesondere die Prägung des in der $C B D$ verwendeten Begriffspaares der ,indigenen und lokalen Gemeinschaft' ergab.

Vor dem Hintergrund der Praxis der überlagernden Anwendung und des gemeinsamen Kerns der drei Konzepte lässt sich juristisch argumentieren, dass alle Konzepte auf ein und dieselbe Gruppe anwendbar sein können. Da alle Konzepte in der Definition der Gruppe denselben Kern aufweisen, kann eine kulturell distinkte Gruppe, die sich als solche identifiziert, unter jedes der völkerrechtlichen Konzepte subsumiert werden. Eine durch die Selbstidentifikation ihrer kulturellen Distinktion getragene Gruppe könnte somit gleichzeitig ,Minderheit', ,indigenes Volk', und ,lokale Gemeinschaft' sein. Diese These der sich überlagernden Konzepte substaatlicher Gruppen setzt jedoch voraus, dass auch die historischen, durch den Regelungskontext geprägten Merkmale, die sich in den Definitionsansätzen niederschlagen, keine sich gegenseitig ausschließende Wirkung entfalten.

\section{Minderheitenschutz für indigene Völker und lokale Gemeinschaften}

Dem internationalen Minderheitenschutz liegt ein Gruppenkonzept zugrunde, welches eine Minderheit anhand der Kriterien einer eigenen Ethnie, Religion und Sprache bzw. einer eigenen Kultur oder Identität sowie eines Ausdrucks des Zusammengehörigkeitsgefühls von der Mehrheitsgesellschaft unterscheiden lässt. Der Begriff ,Minderheit' impliziert dabei begriffsnotwendig das Vorhandensein einer Mehrheit. ${ }^{441}$ Darüber hinaus werden für die Anwendbarkeit von Regelungen zum Minderheitenschutz, insbesondere von Artikel 27 IPbpR, allerdings keine weiteren, qualifizierenden Einschränkungen formuliert.

Bei den europäischen multilateralen Instrumenten werden zusätzlich die Voraussetzungen der Staatsangehörigkeit des Heimatstaates und eine gewisse zeitliche und örtliche Verwurzelung erhoben. Vor dem Hintergrund der politischen Motivation durch diese Voraussetzungen Migranten vom Schutz des Minderheitenrechts auszuschließen, engt sich dadurch zwar positivrechtlich der Anwendungsbereich entsprechender Instrumente ein, es ändert sich aber nichts an der zugrunde liegenden Idee der Gruppe der Berechtigten des internationalen Minderheitenschutzes. Vielmehr bilden diese Voraussetzungen einen politisch gewollten Kontrollmechanismus, der dem Heimatstaat die Entscheidungsgewalt einräumt, wem er auf individueller Basis über die weiteren Regelungen des Einbürgerungsrechts entsprechende Rechte zuerkennen möchte.

Gruppen, die sich durch kulturelle Distinktion sowie eine entsprechende Identifikation und Zusammengehörigkeit von der Mehrheitsgesellschaft differenzieren lassen, sowie quantitativ in ihrem Staat in der Minderzahl sind, können somit den Schutz des Artikel 27 IPbpR genießen. In Europa sind darüber hinaus die Staats-

${ }^{441}$ James Crawford, The Rights of peoples, 1988, S. 60. 
bürgerschaft sowie eine geographische und historische Verwurzelung für die Anwendbarkeit der regionalen Instrumente notwendig. Setzt man dieses Verständnis einer Gruppendefinition des internationalen Minderheitenschutzes in Verhältnis zu den anderen Gruppenkonzepten, so ergeben sich daraus Überlagerungen für die Anwendbarkeit der unterschiedlichen Instrumente.

Das Gruppenkonzept des Minderheitenschutzes lässt eine Anwendung des Artikels 27 IPbpR auch zum Schutz von indigenen Völkern zu. Zwar lehnen es Vertreter von indigenen Gruppen ab, als ethnische Minderheit zu gelten. ${ }^{442}$ Die Praxis des Human Rights Committees zeigt aber, dass Artikel 27 IPbpR dennoch auf indigene Völker Anwendung findet. ${ }^{443}$ In seinem general comment stellt es fest, dass

[...] one or other aspect of the rights of individuals protected under that article - for example, to enjoy a particular culture may consist in a way of life which is closely associated with territory and use of its resources. This may particularly be true of members of indigenous communities constituting a minority. 444

Sofern indigene Gruppen nicht die Mehrheit eines Staates ausmachen, können sie sich somit zum Schutz ihrer Religion, Sprache und kulturellen Identität auf die Instrumente des internationalen Minderheitenschutzes berufen.

Auch im Verhältnis zum Konzept der lokalen Gemeinschaft stellt die Voraussetzung der Minderzahl kein Ausschlusskriterium dar. Die Distinktion lokaler Gemeinschaften beruht auf ihrer mit dem Land verbundenen Wirtschaftsform, welche wesentlicher Bestandteil der Kultur dieser Gruppen ist. Wenn eine lokale Gemeinschaft weniger als die Hälfte der Bevölkerung eines Staates ausmacht, wird sie durch die kulturelle Unterschiedlichkeit ihrer subsistenzwirtschaftlichen Lebensweise vom Minderheitenbegriff umfasst.

Zusammenfassend lässt sich also feststellen, dass der internationale Minderheitenschutz im Sinne des Artikels 27 IPbpR auf indigene Völker sowie auf lokale Gemeinschaften angewendet werden kann, sofern diese Gruppen zahlenmäßig in ihrem Staat in der Minderheit sind. Zusätzlich sind im europäischen Kontext die

${ }^{442}$ Commission on Human Rights, „Advisory Services in the Field of Human Rights, Report on the United Nations Seminar on the effect of racism and racial discrimination on the social and economic relations between indigenous peoples and States", UN Doc. E/CN.4/1989/22, 8. Februar 1989, Abs. 40 (k); So betonte auch der Grand Captain der Mikmaq Indianer, dass seine „tribal society“ keine Minderheit im Sinne von Art. 27 IPbpR sondern vielmehr ein Volk im Sinne von Art. 1 IPbpR sei: UN Generalversammlung, ,,Report of the Human Rights Committee“, Communication No. 78/1980, The Mikmaq Tribal Society v. Canada, UN Doc. A/39/40(Supp), 20. September 1984, Annex XVI, S. 200, Abs. 7.3; Siehe auch: Barsh, „Indigenous Peoples“, S. 835.

443 Alfredsson, „Minority Rights at the United Nations“, S. 23; Kugelmann, „,The Protection of Minorities and Indigenous Peoples Respecting Cultural Diversity“, S. 247, 256.

${ }^{444}$ Human Rights Committee, „General Comment No. 23: The rights of minorities (Art. 27)“, CCPR/C/21/Rev.1/Add.5, Abs. 3.2. 
Voraussetzungen der Staatsangehörigkeit und einer zeitlichen und örtlichen Verwurzelung zu beachten.

\section{Die Indigenität von Minderheiten und lokalen Gemeinschaften}

Die Ansätze einer Definition des Begriffs ,indigene Völker ${ }^{6}$ beziehen sich im Kern auf eine Selbstidentifikation kultureller Distinktion und eine Zusammengehörigkeit der Gruppe mit dem Willen, die eigene Kultur an zukünftige Generationen weitergeben zu wollen. Der Anspruch auf Selbstbestimmung indigener Völker wird unabhängig von ihrer Position und Rolle im gesellschaftlichen Gefüge im Rahmen der etablierten staatlichen Grenzen erhoben, also unabhängig davon, ob ein indigenes Volk eine kleine oder große Gruppe im Staat ausmacht oder eine politisch dominante oder untergeordnete Rolle einnimmt.

Zusätzlich bedingt der historische Hintergrund des Konzepts eine Verbindung zu präkolonialen Gesellschaften, welche den Anwendungsbereich der Rechte indigener Völker - so die Meinung afrikanischer und asiatischer Staaten - auf Länder mit dominierenden Siedlergesellschaften beschränkt. Allerdings zeichnet sich in neueren Entwicklungen ab, dass der Anwendungsbereich des Konzepts ,indigene Völker' nicht auf diese Staaten beschränkt bleibt. ${ }^{445}$ Die Voraussetzung der präkolonialen Verbindung wird vielmehr in eine vergleichbare historische Verwurzelung auf einem bestimmten Territorium uminterpretiert und auf eine vergleichbare Situation der Unterdrückung und Marginalisierung im Verhältnis zu einer dominierenden ,modernen“ Mehrheitsgesellschaft abgestellt.

Insbesondere im Lichte der aktuellen Entwicklungen, ein indigenes Volk nicht nur im Verhältnis zu einer dominierenden europäischen Siedlergesellschaft zu bestimmen, sondern es vielmehr durch eine historische Verwurzelung auf einem bestimmten Gebiet und durch Umstände der Unterdrückung und Marginalisierung auszumachen, lässt für das Konzept der Indigenität eine Auslegung zu, die einer überlagernden Anwendung mit anderen Gruppenrechtsinstrumenten nicht entgegensteht. ${ }^{446}$ So lässt sich argumentieren, dass Minderheiten, sofern sie eine historische und geographische Verbindung zu präkolonialen Kontexten aufweisen oder in einer Situation der Unterdrückung und Marginalisierung leben, im Zuge einer Selbstidentifikation als indigene Völker gelten können.

Auch „lokale Gemeinschaften sind im Verhältnis zu der dominierenden Bevölkerung oft in derselben Situation wie indigene Gruppen und so mit diesen vergleichbar.“447 Der Begriff der lokalen Gemeinschaft gilt als Alternative zum politisch geprägten Begriff der indigenen Völker, da er ohne die Implikationen des umfassenden Anspruchs auf Selbstbestimmung auskommt. Die Offenheit des

\footnotetext{
445 Siehe dazu „B. IV. Regionale Interpretationen in Asien und Afrika“ auf S. 98.

446 von Hahn, Traditionelles Wissen indigener und lokaler Gemeinschaften zwischen geistigen Eigentumsrecbten und der ,public domain", S. 25.

${ }^{447}$ Ebd., 32.
} 
Konzepts ,indigene Völker ‘ wurde während der Verhandlungen der Deklaration zu den Rechten indigener Völker bewusst gewählt, um eine Selbstidentifikation von Gruppen als ,Indigene' in den unterschiedlichsten Kontexten ermöglichen zu können.

Minderheiten und lokale Gemeinschaften können also unter die Bezeichnung ,indigenes Volk ${ }^{6}$ fallen, soweit sie eine Verbindung zu präkolonialen Gesellschaften aufweisen oder sich in einer vergleichbaren Situation der Marginalisierung und Unterdrückung befinden. Entscheidend für die Umsetzbarkeit einer entsprechenden Selbstidentifikation ist dabei vorrangig die Anwendung des Konzepts seitens des Heimatstaats auf seinem Staatsgebiet oder zumindest die regionale Verbreitung des Konzepts bei lokalen und regionalen politischen Akteuren.

\section{Lokale Gemeinschaften bestehend aus indigenen Völkern und Minderheiten}

Lokale Gemeinschaften, wie sie sich im Kontext des internationalen Umweltrechts etabliert haben, sind Gruppen, deren Kultur durch die subsistenzwirtschaftliche Nutzung des Landes geprägt ist, auf dem sie auch historisch verwurzelt sind. Die Konkretisierung des Begriffs ergibt sich insbesondere aus dem Regelungskontext der $C B D$, die in Artikel 8(j) das traditionelle Wissen von Gruppen einer traditionellen, nachhaltigen und Biodiverisität bewahrenden Wirtschaftsweise schützt. Eine überlagernde Anwendung von Regelungen für indigene und lokale Gemeinschaften mit anderen internationalen Gruppenrechtsinstrumenten ist also möglich, sofern diese Gruppen sich in ihrer Kultur ebenfalls durch eine traditionelle Bewirtschaftung ihres Landes auszeichnen.

Dies kann auf indigene Völker zutreffen, da viele indigene Gruppen in traditioneller Art und Weise subsistenzwirtschaftlich Leben. ${ }^{448}$ Die Anerkennung dieser Lebensweise spiegelt sich explizit in der Verwendung des Begriffspaars, indigene und lokale Gemeinschaften' in der CBD wieder. Die umweltvölkerrechtlichen Regelungen sollen gerade auch indigene Völker mit entsprechenden Rechten ausstatten, um so ihre traditionelle Lebensweise zu schützen.

Auch Minderheiten können, sofern sie sich kulturell durch lokale Subsistenzwirtschaft auszeichnen, als, lokale Gemeinschaft' verstanden werden. Neben der kulturellen Distinktion und dem Gefühl der kulturellen Zusammengehörigkeit steht die Minderzahl einer Bezeichnung als solche nicht entgegen. Vielmehr impliziert das Konzept der, lokalen Gemeinschaft' ebenfalls eine gewisse kleine Gruppengröße im Verhältnis zum Rest der Bevölkerung eines Staates.

Sowohl Minderheiten als auch indigene Völker fallen also unter das Konzept der lokalen Gemeinschaft des Umweltvölkerrechts, sofern sie sich durch eine

\footnotetext{
448 Posey, „Identifizierung und Respektierung der Grenzen zwischen indigenen Völkern, traditionellen Bauern und örtlichen Gemeinschaften", S. 247; von Hahn, Traditionelles Wissen indigener und lokaler Gemeinschaften zwischen geistigen Eigentumsrechten und der „public domain“, S. 32.
} 
entsprechende nachhaltige und biodiversitätsfördernde, subsistenzwirtschaftliche Lebensweise auszeichnen.

IV. Überlagernde Anwendbarkeit der drei Konzepte

Alle drei Konzepte rekurrieren in ihren jeweiligen Definitionsansätzen auf eine im Kern vergleichbare Beschreibung der Selbstidentifikation kultureller Distinktion in Bezugnahme auf die völkerrechtlichen Konzepte substaatlicher Gruppen. Zusätzlich prägen die jeweiligen Regelungskontexte weitere Voraussetzungen der unterschiedlichen Konzepte. Sofern eine Gruppe diese zumeist politisch begründeten und teilweise positivrechtlich verankerten Voraussetzungen kumulativ aufweist, können sich auch Überlagerungen in der Anwendung der völkerrechtlichen Instrumente für substaatliche Gruppen ergeben. Aus rechtlicher Sicht ist eine überlagernde Anwendung mehrerer der drei Konzepte substaatlicher Gruppen auf ein und dieselbe Gruppe nicht ausgeschlossen. Eine kulturell distinkte substaatliche Gruppe kann also rechtlich gleichzeitig Minderheit, indigenes Volk und lokale Gemeinschaft sein.

Gruppen von indigenen Völkern oder lokalen Gemeinschaften können also gleichzeitig Minderheiten sein, sofern sie in ihrem Heimatstaat numerisch in der Minderzahl sind. Zur Anwendbarkeit der europäischen Minderheitenschutzinstrumente sind zusätzlich die Staatsbürgerschaft sowie eine historische und geographische Verwurzelung nötig. Auch das Konzept der Indigenität weist heute einen weiten Anwendungsbereich auf. Nach den Anfängen der Indigenenbewegung in den 1970er Jahren in den typischen Siedlerstaaten Nordamerikas, Neuseeland und Australien zeigen aktuelle Entwicklungen, dass lokale Gemeinschaften und Minderheiten auch in Asien und Afrika aufgrund ihrer Marginalisierung und Unterdrückung als indigene Gemeinschaften anerkannt werden können. Des Weiteren ist das Konzept der lokalen Gemeinschaft auch auf Minderheiten oder Indigene anwendbar, sollten diese in ihrer kulturellen Distinktion einer nachhaltigen subsistenzwirtschaftlichen Lebensweise folgen. 


\section{F. Zwischenergebnis}

Die Darstellungen in diesem Teil zeigen die unterschiedlichen Ansätze der Wissenschaft und Praxis die drei völkerrechtlichen Konzepte substaatlicher Gruppen mit rechtlichen, abstrakt-generellen Definitionen greifbar zu machen. Es lassen sich daraus die typischen Bilder und romantisierten Vorstellungen dessen ableiten, was die Bezeichnungen ,Minderheit', ,indigenes Volk' und ,lokale Gemeinschaft' ausmachen sollen.

Typische Minderheiten, wie beispielsweise die Sorben in Deutschland, die Ungarn in Österreich oder die Katalanen in Spanien, zeichnen sich durch eine eigene Sprache und Religion aus, sie tragen traditionelle Trachten und haben zweisprachige Ortsschilder sowie Schulen, auf denen ihre Kinder in der Muttersprache unterrichtet werden. Meistens haben sie eine ethnische Beziehung zu einem Nachbarstaat, mit dem sie gemeinsame kulturelle Wurzeln teilen. Neben diesen kulturellen Unterschieden zeichnen sich Minderheiten durch ihre Minderzahl im Vergleich zur übrigen Bevölkerung ihres Heimatstaates aus.

Das romantisierte Bild indigener Völker zeigt Menschen in traditionellen Behausungen in geschützten Reservaten in Amerika, die mit traditionellen Waffen jagen oder einfachen Ackerbau betreiben und dabei nach überlieferten Gebräuchen leben. Ihre Traditionen und Kultur reichen über mehrere Generationen zurück in die Zeit vor der Kolonisierung und die Besiedelung ihres Landes durch die Europäer. Diese Gruppen erlitten und erleiden eine politische und soziale Marginalisierung und Unterdrückung durch die Kolonisatoren und ihre Nachfahren, die sich nicht nur ihres Landes ermächtigten, sondern sich auch ihr traditionelles Wissen und ihre traditionellen kulturellen Ausdrucksformen aneignen und kommerzialisieren.

Ein ähnliches Bild zeigt die Idealvorstellung lokaler Gemeinschaften. Sie leben autark in abgelegenen Dörfern, in beinahe unberührter Natur, erwirtschaften ihren Lebensunterhalt durch eigenen Ackerbau und die Jagd in naheliegenden Wäldern. Sie nutzen ihr traditionelles Wissen unter anderem zum Erhalt der Vielfalt in Flora und Fauna ihrer unmittelbaren Umgebung. In ihrer subsistenzwirtschaftlichen Lebensweise wirtschaften sie nachhaltig mit den biologischen Ressourcen und bewahren somit die biologische Vielfalt ihrer Umwelt.

Diese idealisierten Bilder von Minderheiten, indigenen Völkern und lokalen Gemeinschaften lassen sich in dieser romantisierten Form wohl kaum in der Wirklichkeit finden. Sie dienen aber, spätestens in der medialen Darstellung dieser Gruppen, als Stereotype und entfalten mit den im Einzelfall dann zu oft falschen Zuschreibungen bestimmter Eigenschaften eine äußerst problematische Wirkung. Den Auswirkungen der Verkennung kollektiver Identitäten in der sozialen Wirklichkeit wird am Ende von Teil 3 mit kultur- und politikwissenschaftlicher Per- 
spektive nachgegangen, um interdisziplinär Rückschlüsse für die normative Ebene zu ziehen. ${ }^{449}$

Die dargestellten Definitionsansätze sollen eigentlich jedoch nur der Beantwortung einer rechtlichen Frage dienen: Fällt eine kulturell distinkte substaatliche Gruppe unter eines der Konzepte und kann somit entsprechende Rechte für sich geltend machen?

Entscheidend sind Gestaltung und Anwendung rechtlicher Definitionen somit in den Grenzfällen, wenn sich neue Gruppen bilden oder neue Instrumente implementiert werden sollen. So stellen sich die Probleme beispielsweise bei Fragen nach dem Minderheitenstatus der seit Jahrzehnten in Deutschland lebenden Polen oder Türken, nach den Hintergründen der Casinolizenzen neu registrierter Indianerstämme in den USA, nach, neuen' indigenen Völkern in Afrika und Asien oder nach der notwendigen Beteiligung und Zustimmungsbefugnis lokaler Gemeinschaften zum bioprospecting.

Die Diskurse um juristische Definitionen der Konzepte substaatlicher Gruppen konnten auf internationaler Ebene bisher allerdings keinen Konsens finden. Das zeigen die in diesem Teil dargestellten Ansätze, die zwar alle für idealtypische, kulturell distinkte Gruppen unproblematisch sein mögen, für die Grenzfälle jedoch sehr umstritten sind. Mangels Einigung auf internationaler Ebene wird für die Implementierung der unterschiedlichen Rechtsinstrumente immer auf eine Ausgestaltung der Konzepte auf nationaler Ebene verwiesen. In Anbetracht der weltweiten Vielfalt kulturell distinkter Gruppen sei es nicht möglich, sich auf global anwendbare Definitionen zu einigen, ist die häufig vorgebrachte Begründung. Es gibt bis heute auf internationaler Ebene keine Primärquellen mit globaler Verbreitung, welche den Konsens der Staatengemeinschaft zur Frage der Anwendbarkeit der unterschiedlichen Instrumente für substaatliche Gruppen in rechtlicher Weise ausdrücken. Grundsätzlich, so das Ziel der bisherigen Definitionsdebatten, soll aber auf Basis rechtlicher Argumente eine unabhängige Beurteilung über den rechtlichen Status substaatlicher Gruppen stattfinden können, damit ihre Rolle im staatlichen und internationalen Kontext nicht von politischen Erwägungen abhängt. Angesichts der Diskussionen um die verschiedenen Definitionsansätze, von denen keine bisher globalen Konsens gefunden hat, ist nach wie vor offen wie eine abstrakt-generelle rechtliche Lösung aussehen könnte.

${ }^{449}$ Siehe dazu: „C. Interdisziplinäre Perspektiven: Konstruktivismus und Anerkennung“ auf S. 163. 



\section{Teil 3: Anerkennung}

Mangels völkerrechtlich geltender, abstrakt-genereller Definitionen ist weiterhin offen, wie die Anwendbarkeit der drei Konzepte substaatlicher Gruppen rechtlich zu Regeln ist. Es ist rechtlich nicht geklärt, welche Gruppe eine Minderheit, ein indigenes Volk oder eine lokale Gemeinschaft sein und entsprechende Rechte beanspruchen kann. Die Anwendung der Konzepte, so zeigt es die Praxis, erfolgt letztlich einzelfallbezogen. Mangels rechtsverbindlicher Definitionen entscheidet sich die Frage, ob eine Gruppe unter eines der drei Konzepte fällt, also nicht durch juristische Subsumtion ausgewählter Merkmale. Die Regelungen zum Minderheitenschutz, für die Selbstbestimmung indigener Völker und die Berücksichtigung lokaler Gemeinschaften im Umweltrecht werden von unterschiedlichsten Akteuren auf nationaler und internationaler Ebene in der Praxis auf bestimmte Gruppen angewendet und diese dadurch implizit als ,Minderheit', ,indigenes Volk ${ }^{\varsigma}$ oder , lokale Gemeinschaft ${ }^{\varsigma}$ anerkannt. Dieser Anerkennung substaatlicher Gruppen, die in der Praxis den Gruppen ihren jeweiligen Gruppenrechtsstatus verleiht, hat neben den rechtswissenschaftlichen Debatten um Definitionen bisher jedoch keine Beachtung gefunden. Ausgehend von der Praxis soll hier die Anerkennung substaatlicher Gruppen in ihrer rechtlichen Bedeutung untersucht werden. Ziel ist es, den normativen Gehalt der Anerkennung substaatlicher Gruppen deutlich zu machen und sie als analytische Perspektive für die Praxis der Implementierung der Rechte substaatlicher Gruppen 
zu verwenden. Die Anerkennung substaatlicher Gruppen, verstanden als Ergebnis eines Prozesses, ist eine tragfähige Lösung der in Teil 2 aufgezeigten Definitionsproblematik. Sie wirkt als Pendant zur Selbstidentifikation, lässt sich rechtlich einordnen und interdisziplinär mit Kultur- und Politikwissenschaften verknüpfen. Die bisher erfolglosen Debatten über Definitionen kulturell distinkter substaatlicher Gruppen können so obsolet werden.

\section{A. Anerkennungsverfahren: eine Auswahl}

Grundlage für eine rechtliche Bewertung der Implementierung der Rechte substaatlicher Gruppen als Anerkennungsverfahren ist die Praxis der Umsetzung der einschlägigen Rechtsinstrumente. Im Folgenden soll daher eine Auswahl dieser Praxis unter einer Perspektive der Anerkennung dargestellt werden, um auf dieser Grundlage den normativen Gehalt der Anerkennung substaatlicher Gruppen erarbeiten zu können.

Für eine rechtliche Anerkennung von substaatlichen Gruppen und den damit einhergehenden Fragen zu Zuständigkeit, Voraussetzungen, Prozedere und Rechtsfolgen sind völkerrechtlich keine Vorgaben gemacht. Ebenso wenig finden sich Diskussionen zu Anerkennungsprozessen in den bisherigen Debatten um Definitionen in der Völkerrechtspraxis und -wissenschaft. Wenn substaatliche Gruppen gegenüber ihren Heimatstaaten auftreten, obliegt es dem jeweiligen nationalen politischen Prozess, wie die Umsetzung völkerrechtlicher Instrumente in Form von Verfahren und Definitionen - also die Voraussetzungen der Anerkennung von substaatlichen Gruppen - auf lokaler Ebene ausgestaltet wird. Folglich finden sich weltweit unterschiedliche Institutionen und Regelungen, wie sich einzelne Gruppen selbst als ,Minderheit', indigenes Volk' oder ,lokale Gemeinschaft identifizieren und für diesen Status von Seiten ihrer Heimatstaaten um Anerkennung suchen.

Mangels eines international verbindlichen Rechtsrahmens ergeben sich ebenfalls erhebliche Spielräume, wie unterschiedliche nationale und internationale, zwischenstaatliche und nicht-staatliche Akteure mit substaatlichen Gruppen interagieren. Beispielsweise entwickelte die Weltbank ihre eigenen Voraussetzungen für den Umgang mit indigenen Völkern, Vertreter indigener Gemeinschaften organisieren sich in transnationalen NGOs, unbenommen einer Anerkennung durch ihre Heimatstaaten und unabhängige Wissenschaftler definieren für ihre anthropologische Forschung ihr Feld indigener Völker oder lokaler Gemeinschaften.

Es entwickelten sich eine Reihe unterschiedlicher Verfahren und Voraussetzungen, die von Seiten der beteiligten Akteure - seien dies Regierungen, internationale Organisationen, NGOs oder gar Einzelpersonen - aufgestellt werden. Zusätzlich beeinflussen sich unterschiedliche Anerkennungsverfahren der verschiedenen Ebenen: Ist eine Gruppe international als Minderheit anerkannt, bewirkt dies möglicherweise eine entsprechende Anerkennung von Seiten des Heimatstaa- 
tes im nationalen Kontext. Konstatiert ein Staat die Indigenität einer Gruppe, wird diese in der Regel auch als solche auf internationaler Ebene behandelt. Allerdings sind auch diese Verknüpfungen und Auswirkungen der Anerkennung durch die Bezugnahme auf andere Foren und Institutionen, ihre Prozesse und individuellen Konkretisierungen der Definitionen substaatlicher Gruppen nicht allgemein geregelt und müssen im Einzelfall untersucht werden.

Da die Masse an unterschiedlichen Akteuren und das Netz sich daraus ergebender Beziehungen, also alle Verbindungen zwischen substaatlichen Gruppen mit ihren Heimatstaaten, Drittstaaten, internationalen Organisationen, NGOs und privaten Akteuren und diese wiederum untereinander in Bezug auf die Gruppen, hier nicht global umfassend untersucht werden können, soll im Folgenden eine Auswahl von Verfahren, Strukturen und Instrumenten der Anerkennung - und auch der Nicht-Anerkennung - dargestellt werden.

\section{Minderheiten}

Im internationalen Minderheitenschutz sind es vor allem die Staaten, welche die Schlüsselrolle bei der Umsetzung und Anwendung von Minderheitenrechten auf ihrem Territorium spielen. Im Rahmen der Berichts- und Beschwerdemechanismen nehmen aber auch internationale Institutionen entscheidenden Einfluss. Es zeigen sich somit die Folgenden unterschiedlichen Verfahren und Akte der Anerkennung von Minderheiten.

\section{Regelungen zu Minderheiten in bilateralen Verträgen}

Die Situation veränderter Grenzziehung nach dem Ersten Weltkrieg machte es erforderlich, dass die europäischen Staaten Regelungen zum Schutz von Minderheiten trafen. Da sich die Siegermächte nicht auf einen umfassenden multilateralen Ansatz einlassen wollten, wurden in einer Vielzahl von bilateralen Minderheitenschutzverträgen Regelungen zu Sprachrechten, Staatsangehörigkeit und Migration getroffen. Diese bilateralen Verträge aus der Zeit des Völkerbundes implizierten dabei die Anerkennung der Existenz einer Minderheit auf dem eigenen Staatsgebiet, die eine ethnische Verbindung zum Vertragspartner aufwies. Auch die modernen bilateralen Verträge zum Minderheitenschutz beinhalten eine entsprechende Anerkennung von Minderheiten der ethnischen Herkunft der beiden Vertragsparteien. Teilweise werden in den modernen Verträgen konkrete Minderheiten auch explizit benannt und ihre Existenz somit individuell anerkannt. 450

450 Arp, International norms and standards for the protection of national minorities, S. 27. 


\section{Der Einfluss multilateraler Verträge auf die Anerkennung von Minderheiten}

Bei multilateralen Minderheitenschutzbestimmungen wie dem IPbpR oder den europäischen Instrumenten, lässt sich der Abschluss der Verträge nicht als implizite Anerkennung individueller Minderheiten interpretieren. Es sind vielmehr Akte der Staatsorgane, die eine Anerkennung explizit oder implizit ausdrücken.

Dies zeigt das Beispiel der Anerkennung der Fahrenden in der Schweiz. Schon kurz vor der Ratifikation des Rabmenübereinkommens zum Scbutz, nationaler Minderheiten des Europarats im Oktober 1998 hat der Schweizer Bundesrat in einer Botschaft an das Parlament ausdrücklich festgehalten, dass die schweizerischen Fahrenden eine nationale Minderheit im Sinne des Rahmenübereinkommens bilden. ${ }^{451}$ Zum Hintergrund dieser Anerkennung wird auf die langjährige finanzielle Unterstützung des Dachverbands der schweizerischen Fahrenden von Seiten des Bundes verwiesen sowie die Bedeutung der Selbstidentifikation der Gruppe anerkannt. So wurde unter anderem auf die gleichzeitige Anwendung der ILO Convention No. 169 verwiesen, nach der „die Selbstdefinition einer sozialen Gruppe für die Frage der Anwendbarkeit dieses Abkommens eine wichtige Rolle" spiele. 452 Dieser offene Umgang mit der Selbstidentifikation von Minderheiten und eine fast als Automatismus erscheinende Anerkennung von Seiten der schweizerischen Regierung ist in Europa jedoch eher die Ausnahme als die Regel.

Dies zeigt das Beispiel Deutschlands. Explizit erkennt die Bundesrepublik die Sorben und Dänen als nationale Minderheiten an und wendet die internationalen Schutzbestimmungen, wie beispielsweise das Rabmenübereinkommen zum Schutz nationaler Minderheiten des Europarates, auch auf die Friesen sowie die Sinti und Roma deutscher Staatsangehörigkeit an. ${ }^{453}$ Die Sinti und Roma wurden erst nach einem jahrzehntelangen Prozess politischer Bürgerbewegung, der bis in die späten 1970er Jahre zurückreicht, als Minderheit anerkannt. ${ }^{454}$ Weiterhin bemühen sich die Polen sowie die Roma aus dem Kosovo ohne deutsche Staatsangehörigkeit um eine Anerkennung als nationale Minderheit. So stellt der Bund der Polen auf seiner Website entsprechende Dokumente zusammen, die den Status der Polen als nationale Minderheit in Deutschland begründen sollen. ${ }^{455}$ Auf den Hinweis des Beratenden Ausschusses zum Rahmenübereinkommen, der die schwierige Situation der Roma aus dem Kosovo in Deutschland kritisiert, weist Deutschland „ausdrücklich und wiederholt darauf hin, dass es sich bei dem zitierten Personenkreis

\footnotetext{
451 Bundesrat der Schweizerischen Eidgenossenschaft, „Botschaft über das Rahmenübereinkommen des Europarates zum Schutz nationaler Minderheiten“, BBl 1998 II, 1293, 19. November 1997, S. 1310 .

452 Bundesamt für Kultur, „Anerkennung als nationale Minderheit“, 14. August 2007.

453 Germany, „Declarations made upon Signature“, Framework Convention for the Protection of National Minorites, 2141 UNTS 271.

454 Dokumentations- und Kulturzentrum Deutscher Sinti und Roma, „Bürgerrechtsbewegung“.

455 Bund der Polen in Deutschland e.V., zugegriffen 8. Januar 2017, http:/ /www.zpwn.org.
} 
nicht um deutsche Staatsangehörige handelt und dieser Personenkreis somit auch nicht in den Mandatsbereich des Beratenden Ausschusses für das Rahmenübereinkommen fällt. “456 Auch den Vorschlag, das Rahmenübereinkommen nur artikelweise auf sie und weitere Gruppen anzuwenden, lehnte die Bundesregierung in einer Stellungnahme vom Juli 2002 ausdrücklich ab. ${ }^{457}$ Dennoch befasst sich der Beratende Ausschuss zum Rahmenübereinkommen mit den kosovarischen Roma in Deutschland, erkennt sie somit implizit als Minderheit an und wirkt politischen Druck auf Deutschland aus, es ihm nachzutun.

Ähnlich zeigt der generelle Vorbehalt Frankreichs zur Anwendbarkeit des Artikels 27 IPbpR die grundsätzlich defensive Politik europäischer Staaten bei der Anerkennung von Minderheiten. ${ }^{458}$

\section{Internationale Institutionen}

Auch auf internationaler Ebene zeigen sich die Auseinandersetzungen mit der Unbestimmtheit der Definitionsansätze und der Notwendigkeit der Anerkennung individueller Gruppen als Minderheiten. So stellt beispielsweise einerseits das Human Rights Committee fest, dass „,[t]he existence of an ethnic, religious or linguistic minority in a given State party does not depend upon a decision by that State party but requires to be established by objective criteria. " ${ }^{459}$ Andererseits wurde auf dem Expertentreffen unter Esbjørn Eide 1993 von einigen Teilnehmern proklamiert, dass die Probleme des Minderheitenschutzes eher pragmatisch angegangen werden sollten als nach einer allgemeingültigen Definition zu suchen. ${ }^{460}$ In der Praxis zeigt sich, dass der Ausschuss seit der Veröffentlichung seiner comments zu den Staatenberichten nach dem Zusammenbruch des Ostblocks die Regelungen des Artikels $27 \mathrm{IPbpR}$ auch in solchen Staaten anwendet, in denen die Existenz von Minderheiten von offizieller Seite verneint wird. 461

456 Advisory Committee on the Framework Convention for the Protection of National Minorities, „Dritte Stellungnahme zu Deutschland Verabschiedet am 27. Mai 2010 sowie Stellungnahme Deutschlands zum Dritten Bericht des Beratenden Ausschusses für das Rahmenübereinkommen zum Schutz nationaler Minderheiten (2. Dezember 2010)“, ACFC/OP/III (2010) 003, 27. Mai 2010, Abs. 93 und „Zu Rn 93“, S. 22 und 53.

${ }^{457}$ Bundesministerium des Innern, „Stellungnahme der Bundesrepublik Deutschland zu der Stellungnahme des Beratenden Ausschusses zu dem Bericht über die Umsetzung des Rahmen Übereinkommens zum Schutz nationaler Minderheiten in der Bundesrepublik Deutschland“, Juli 2002, S. 7.

458 Thornberry, „The UN Declaration on the Rights of Persons Belonging to National or Ethnic, Religious and Linguistic Minorities: Background, Analysis, Observations, and an Update", S. 21.

${ }^{459}$ Human Rights Committee, „General Comment No. 23: The rights of minorities (Art. 27)“, CCPR/C/21/Rev.1/Add.5, Abs. 5.2.

${ }^{460}$ Commission on Human Rights, , Rights of Persons belonging to National or Ethnic, Religious and Linguistic Minorities“, UN Doc. E/CN.4/1993/85, 10. Februar 1993, Abs. 6, $17 \mathrm{f}$.

${ }^{461}$ Scherer-Leydecker, Minderheiten und sonstige ethnische Gruppen, S. $92 \mathrm{f}$. 
Im Rahmen der OSZE befasste sich der Hohe Kommissar für nationale Minderbeiten mit der Frage der Bestimmung von Minderheiten und stellte dazu in seiner keynote bei der Commission on Security and Cooperation in Europe im Mai 1993 fest, dass

$[\ldots]$ the existence of a minority is a question of fact and not of definition. [...] To belong to a national minority is a matter of a person's individual choice. [...] I would dare to say that I know a minority when I see one. ${ }^{462}$

Der Hohe Kommissar für nationale Minderheiten legt seiner Arbeit einen weiten Definitionsansatz zugrunde und untersucht Fälle ungeachtet der von den Staaten geforderten Voraussetzung der Staatsangehörigkeit des Heimatstaates der einzelnen Mitglieder einer Minderheit. ${ }^{463}$

Der Europäische Gerichtshof für Menschenrechte hat Fragen der Anerkennung von Minderheiten bisher immer ausweichend als Fragen des Rechts auf Achtung des Privatlebens (Artikel 8 EMRK) und der Versammlungs- und Vereinigungsfreiheit (Artikel 11 EMRK) behandelt. ${ }^{464}$ Auch im Fall Gorzelike v Poland, in dem der ethnischen Minderheit der Schlesier eine Vereinsgründung mit der Bezeichnung ,schlesische nationale Minderheit' in ihrer Satzung versagt wurde, kam der EGMR zu keiner Entscheidung bezüglich abstrakt-genereller Voraussetzungen einer ,nationalen Minderheit ${ }^{6}{ }^{465}$ Es liegt in der margin of appreciation und stellt keine unverhältnismäßige Entscheidung der polnischen Behörden und Gerichte dar, wenn sie einer, ethnischen Minderheit ${ }^{\star}$ eine Bezeichnung als solche und somit etwaige Sonderrechte einer ,nationalen Minderheit', die so im Rahmen der Wahlgesetze allgemein angelegt sind, versagen. ${ }^{466}$ Der EGMR gesteht somit den nationalen Behörden und Gerichten einen Ermessensspielraum bei der Anerkennung des Rechtsstatus als, nationale Minderheit ${ }^{\natural} \mathrm{zu}$, der in der Folge entsprechende Sonderrechte im Wahlrecht des Landes impliziert.

\footnotetext{
462 Max van der Stoel, OSCE High Commissioner on National Minorities, „Case Studies on National Minority Issues: Positive Results, Keynote Address to the CSCE Human Dimension Seminar, 24 May 1993, Warsaw, Poland“, in: Wolfgang Zellner und Falk Lange (Hrsg.), Peace and Stability through Human and Minority Rights, 2001, S. 44.

${ }_{463}$ Scherer-Leydecker, Minderheiten und sonstige ethnische Gruppen, S. 191.

${ }^{464}$ Hofmann, „The Framework Convention for the Protection of National Minorities“, S. 49f.

465 Europäischer Gerichtshof für Menschenrechte, „Case of Gorzelik and other v. Poland (44158/98), Grand Chamber“, Reports of Judgments and Decisions / Recueil des Arrêts et Décisions, 2004-I, 17. Februar 2004, S. 219.

466 Ebd., S. 265; Hofmann, „The Framework Convention for the Protection of National Minorities“, S. $50-52$.
} 


\section{Zusammenfassung: die Politik der Anerkennung von Minderbeiten}

Der Überblick über die Praxis der Anerkennung von Minderheiten zeigt, dass unterschiedliche Verfahren und Akteure in den jeweiligen nationalen Kontexten und auch auf internationaler Ebene für die Umsetzung und Überwachung der Minderheitenschutzinstrumente entscheidend sind. Mangels rechtlich festgelegter Verfahren ergibt sich eine Vielzahl von Varianten des Zusammenspiels der unterschiedlichen Akteure, die unterschiedlich schnell und in unterschiedlichen Formen der Beteiligung der Gruppen sowie der Mehrheit schließlich die Existenz einer ,Minderheit' anerkennen oder eine Anerkennung verweigern.

In den Debatten um diese Problematik wird vorgebracht, die mangelnde Schärfe der Definitionsansätze sei zu begrüßen, da somit ein flexibler Umgang im Prozess der Anerkennung möglich sei. ${ }^{467}$ Dabei sind es aber trotz der Initiativen unterschiedlicher Akteure letztlich die Heimatstaaten, in denen die Gruppen leben, die die Entscheidungsmacht besitzen, Gruppen als ,Minderheit anzuerkennen oder nicht. Dies zeigt das Beispiel der Anregung des Beratenden Ausschusses zum Rahmenübereinkommen gegenüber Deutschland: Der Minderheitenschutz solle auch den nicht-deutschen Staatsbürgern der Sinti und Roma aus dem Kosovo gewährt werden, so der Vorschlag, ${ }^{468}$ der jedoch mit einer ausdrücklichen Absage beschieden wurde. ${ }^{469}$

Ansätze einer wissenschaftlichen Weiterentwicklung theoretischer Gruppenrechtskonzepte versuchen, die „Macht des Normativen“ diesen faktischen Entscheidungen entgegenzusetzen, um so der „Willkür der einzelnen Staaten“ zu begegnen. ${ }^{470}$ Schließlich wird vertreten, die Anerkennung von Seiten des Staates sei keine Voraussetzung für die Existenz einer Minderheit, dies sei vielmehr eine empirische Frage. ${ }^{471}$ „If the existence of a minority group within a state is objectively demonstrated, non-recognition of the minority does not dispense the State from the duty to comply with the principles in article $27^{\prime \prime}$ IPbpR. 472 Die Vielfalt verschiedener Verfahren und unterschiedliche rechtliche Beurteilungen der Be-

467 Drzewicki, „Minority Protection within the OSCE“, S. 101.

468 Advisory Committee on the Framework Convention for the Protection of National Minorities, „Dritte Stellungnahme zu Deutschland Verabschiedet am 27. Mai 2010 sowie Stellungnahme Deutschlands zum Dritten Bericht des Beratenden Ausschusses für das Rahmenübereinkommen zum Schutz nationaler Minderheiten (2. Dezember 2010)“, ACFC/OP/III (2010) 003, Abs. 93 und „Zu Rn. 93“, S. 22, 53.

${ }^{469}$ Bundesministerium des Innern, „Stellungnahme der Bundesrepublik Deutschland zu der Stellungnahme des Beratenden Ausschusses zu dem Bericht über die Umsetzung des Rahmen Übereinkommens zum Schutz nationaler Minderheiten in der Bundesrepublik Deutschland“, S. 7.

470 Roßkopf, Theorie des Selbstbestimmungsrechts und Minderheitenrechts, S. 14.

471 Nowak, U.N. covenant on civil and political rights, Art. 27, Rn. 22.

472 Sub-Commission on the Prevention of Discrimination and Protection of Minorities, Francesco Capotorti, „Study on the rights of persons belonging to ethnic, religious, and linguistic minorities", UN Doc. E/CN.4/Sub.2/384/Rev.1, Abs. 570. 
deutung der Anerkennung verdeutlicht, dass die Politik bei der Vergabe des Minderheitenstatus' eine erhebliche Rolle spielt.

\section{Indigene Völker}

Für indigene Völker finden sich neben ihrer mittlerweile etablierten Rolle auf internationaler Ebene auch verschiedene Institutionen auf nationaler Ebene, die für den Prozess der Anerkennung eine entscheidende Rolle spielen.

\section{Staatliche Anerkennung indigener Völker}

In den Vereinigten Staaten hat das für die US-amerikanischen indigenen Völker zuständige Bureau of Indian Affairs seit 1824 die Aufgabe

[to] enhance the quality of life, to promote economic opportunity, and to carry out the responsibility to protect and improve the trust assets of American Indians, Indian tribes, and Alaska Natives. ${ }^{473}$

Das Bureau ist in diesem Sinne für 566 anerkannte amerikanische Indianer, indianische Stämme und alaskasche Eingeborene, insgesamt also für ca. 1,9 Millionen Indigene zuständig, verwaltet 55 Millionen Morgen Land sowie 57 Millionen Morgen unterirdischer Bodenschätze und stellt Bildungseinrichtungen für ca. 42.000 indianische Schüler und Studenten zur Verfügung. Unter anderem erteilt es ebenfalls die Lizenzen für Spielcasinos, wie zuletzt im Dezember 2011 für die Keweenaw Bay Indian Community. ${ }^{474}$

Das Bureau for Indian Affairs ist auch für die Registrierung von ,neuen' Stämmen zuständig, also von Stämmen, die seit jeher existieren aber von staatlicher Seite noch keine Anerkennung als indigenous peoples gefunden haben. Historisch wurden bisher die meisten Stämme durch Verträge, den Kongress, executive orders des Präsidenten, andere Bundesverwaltungsakte oder Entscheidungen von Bundesgerichten anerkannt. 1978 hat das Innenministerium die gesetzlichen Regelungen überarbeitet und das Gesetz 25 C.F.R. Part $83.7 \mathrm{zu}$ den Voraussetzungen neuer Anerkennungen erlassen. Dieses wurde 1994 reformiert und mit dem Federally Recognized Indian Tribe List Act ergänzt. ${ }^{475}$ Seitdem können indianische Gruppen ihre Anerkennung als federally recognized tribe nur noch durch eine Entscheidung des Kongresses, einen Verwaltungsakt unter den Voraussetzungen des 25 C.F.R. Part

\footnotetext{
473 Donald L. Fixico, „Bureau of Indian Affairs“, 2012, S. xiii.

${ }^{474}$ US Department of the Interior, Office of the Assistant Secretary - Indian Affairs (Press Release), „Echo Hawk Issues Decisions on Two Tribal Gaming Applications“, 20. Dezember 2011.

475 Department of the Interior, Bureau of Indian Affairs, „Procedures for establishing that an American Indian Group exists as an Indian Tribe“, 59 FR 9293, 25. Februar 1994, 25 C.F.R. 83.7, „Mandatory criteria for Federal acknowledgment“, S. 9295; US Congress, „,The Federally Recognized Indian Tribe List Act of 1994“, Public Law 103-454, 108 Stat. 4791, 2. November 1994.
} 
83.7 oder einen Gerichtsentscheid erhalten. ${ }^{476}$ Auf Grundlage dieser Regelungen wird jährlich das Bundesregister der federally recognized tribes veröffentlicht. ${ }^{477}$ So wurde beispielsweise nach einem „32-year battle for federal recognition“ im Oktober 2010 die Shinnecock Indian Nation im Staat New York anerkannt, die nun das Verfahren für eine Casinolizenz vorantreibt. ${ }^{478}$

Die gesetzlichen Vorschriften des 25 C.F.R. Part 83.7 regeln neben den Voraussetzungen zur Anerkennung auch zulässige Beweismittel. Insgesamt müssen für die Anerkennung demnach folgende Voraussetzungen erfüllt sein:

(a) The petitioner has been identified as an American Indian entity on a substantially continuous basis since 1900 .

(b) A predominant portion of the petitioning group comprises a distinct community and has existed as a community from historical times until the present.

(c) The petitioner has maintained political influence or authority over its members as an autonomous entity from historical times until the present.

(d) A copy of the group's present governing document including its membership criteria. In the absence of a written document, the petitioner must provide a statement describing in full its membership criteria and current governing procedures.

(e) The petitioner's membership consists of individuals who descend from a historical Indian tribe or from historian Indian tribes which combined and functioned as a single autonomous political entity.

(f) The membership of the petitioning group is composed principally of persons who are not members of any acknowledged North American Indian tribe.

(g) Neither the petitioner nor its members are the subject of congressional legislation that has expressly terminated or forbidden the Federal relationship. ${ }^{479}$

\footnotetext{
476 US Congress, „The Federally Recognized Indian Tribe List Act of 1994“, Public Law 103-454, 108 Stat. 4791, Sec. 103, Abs. 3.

477 Ebd., Sec. 103, Abs. 6-8; Siehe beispielsweise: Department of the Interior, Bureau of Indian Affairs, „Indian Entities Recognized and Eligible To Receive Services From the United States Bureau of Indian Affairs“, 81 FR 26826, 4. Mai 2016.

${ }^{478}$ Danny Hakim, „U.S. Recognizes Shinnecock Tribe on Long Island“, New York. Times, 15. Juni 2010; Department of the Interior, Bureau of Indian Affairs, „Final Determination for Federal Acknowledgment of the Shinnecock Indian Nation“, 75 FR 34760, 18. Juni 2010.

479 Department of the Interior, Bureau of Indian Affairs, ,Procedures for establishing that an American Indian Group exists as an Indian Tribe“, 59 FR 9293, 25. Februar 1994, 25 C.F.R. 83.7, „Mandatory criteria for Federal acknowledgment“, S. 9295.
} 
Als Beweismittel kann die Identifizierung als American Indian entity durch Anthropologen, Historiker oder andere Wissenschaftler erfolgen oder die „identification as an Indian entity in newspapers and books" beigebracht werden. ${ }^{480} \mathrm{Um}$ die Distinktion der Gemeinschaft zu belegen, können unter anderem

(iii) Significant rates of informal social interaction which exist broadly among the members of a group.

(iv) A significant degree of shared or cooperative labor or other economic activity among the membership.

(v) Evidence of strong patterns of discrimination or other social distinctions by non-members ${ }^{481}$

vorgebracht werden. Zum Beleg der Abstammung von einem historischen Stamm können unter anderem auch Dokumente der Kirchen oder von Schulen und anderen Archiven sowie eidesstattliche Erklärung von Stammesältesten, Stammesführern oder der Selbstverwaltungsorgane vorgelegt werden. ${ }^{482}$

Auch wenn das Verfahren für jeden einzelnen Stamm insgesamt sehr teuer und langwierig sein kann und bei der Würdigung der einzelnen Beweise immer noch ein Ermessensspielraum von Seiten des Bureau of Indian Affairs gegeben ist, geben die gesetzlichen Regelungen zumindest einen konkreten Rahmen, die zuständigen Stellen für einen Anerkennungsprozess sowie Regelungen zu zulässigen Beweismitteln vor. 483

\section{Internationale Organisationen und indigene Völker}

Seit den 1970er Jahren ist die globale Indigenenbewegung auf internationaler Ebene präsent. Die Aktivitäten indigener Völker liegen in der Teilnahme als Beobachter verschiedener internationaler Staatenkonferenzen sowie institution alisiert als Beratungsgremium des ECOSOC im UN Permanent Forum on Indigenous Issues. Indigene Völker sind Gegenstand der unterschiedlichen Berichtsverfahren und treten als Beschwerdeführer vor den Institutionen der verschiedenen Menschenrechtsinstrumente auf. Sie interagieren unmittelbar mit internationalen Organisationen und Institutionen, wie beispielsweise der Weltbank oder der Asian Development Bank, bei der Umsetzung konkreter Projekte. In allen diesen Kontexten ergeben sich jeweils verschiedene Prozesse einer Anerkennung ihres Status' als indigenes Volk mit je unterschiedlichen Voraussetzungen.

Das UNPFII konstituiert sich aus 16 Experten sowie einer jährlich zweiwöchigen Sitzung, bei der eine Vielzahl von Vertretern indigener Völker sowie Regie-

\footnotetext{
${ }^{480}$ Ebd., Abs. a) (4), (5).

${ }^{481}$ Ebd., Abs. b) (1) (iii)-(v).

482 Ebd., Abs. e) (1) (iii), (iv).

${ }^{483}$ M. Maureen Murphy, „The Bureau of Indian Affairs' Process for Recognizing Groups as Indian Tribes“,CRS Report for Congress, 25. März 2005.
} 
rungsvertreter, Vertreter internationaler Organisationen und Vertreter anderer NGOs zusammen kommen. ${ }^{484}$ Für die Teilnahme von Organisationen indigener Völker bedarf es einer pre-registration, um die für den Visumsantrag für die USA notwendige formelle Einladung zu erhalten. Zugelassen zur pre-registration werden drei Kategorien:

(1) indigenous peoples' organizations;

(2) non-governmental organizations in consultative status with ECOSOC; and

(3) academic institutions. ${ }^{485}$

Die Kosten der Teilnahme an den Sessions des UNPFII, also die Finanzierung der notwendigen Reiseunterlagen, Flug und Unterkunft müssen die Vertreter selber tragen. ${ }^{486}$ Zur Unterstützung steht allerdings der United Nations Voluntary Fund for Indigenous Populations zur Verfügung, der vom UN High Commissionar for Human Rights verwaltet wird. Einschränkungen zur Teilnahme am UNPFII als Indigenous Peoples' Organization ergeben sich somit nur aus den eigenen finanziellen Möglichkeiten und den zur Verfügung stehenden Plätzen in New York. Eine Selbstidentifikation und Anmeldung als Organisation oder Vertretung eines indigenen Volkes ist ansonsten grundsätzlich ausreichend.

Für die Teilnahme von Vertretern von Organisationen indigener Völker an den Sitzungen des ECOSOC oder des WIPO IGC487 ist eine Registrierung oder Akkreditierung im Rahmen des Verfahrens für alle Vertreter von NGOs notwendig. ${ }^{488}$ Bei diesen Verfahren entscheiden jeweils letztlich die Staaten, welche Organisationen sie zulassen und geben auch die Rahmenbedingungen einer möglichen finanziellen Unterstützung vor. ${ }^{489}$ Zwar geht es dabei, wie auch bei der preregistration für die Sitzungen des UNPFII, formell nur um die Zulassung von NGOs als Interessenvertreter. Die Anerkennung einer NGO als Vertreter einer indigenen Gruppe impliziert aber gleichzeitig indirekt die Anerkennung der Gruppen als ,indigene Völker ${ }^{6}$ auf einer konkret-individuellen Basis durch die Entscheidung eines Komitees oder einer umfassend repräsentativen Gruppe der Staatengemeinschaft.

\footnotetext{
484 Siehe dazu beispielsweise die Liste der Teilnehmer der zehnten Session 2011: Economic and Social Council, Permanent Forum on Indigenous Issues, „Attendance at the ninth session of the Permanent Forum on Indigenous Issues“, UN Doc. E/C.19/2011/INF/1, 2. Juni 2011.

485 UN Permanent Forum on Indigenous Issues, „Handbook for Participants“, 2007, S. 25.

486 Ebd., S. 25-28.

487 Siehe dazu „A. III. 2. Anerkennung durch internationale Organisationen“ auf S. 140.

488 UN Department of Economic and Social Affairs, „How to Apply for Consultative Status“; WIPO IGC, „Participating in the IGC“.

489 WIPO Generalversammlung, „Establishment of the WIPO Voluntary Fund for Accredited Indigenous and Local Communities“, Annex zu WO/GA/32/6, 24. August 2005, geändert durch WO/GA/39/11, 5. Juni 2010, Art. 5-8.
} 
Eine ausdrückliche Anerkennung von Gruppen als indigene Völker von Seiten internationaler Organisationen erfolgt im Rahmen der verschiedenen Berichtsverfahren der unterschiedlichen Menschenrechtsinstrumente. So setzte beispielsweise die African Commission on Human and Peoples' Rights im Jahr 2000 eine Working Group of Experts on Indigenous Populations/Communities in Africa ein, die 2003 ihren ersten Bericht zu indigenen Populationen/Gemeinschaften veröffentlichte, darin explizit bestimmte Gruppen benennt und ihre Situation diskutiert. ${ }^{490}$ Auch in den Berichten der UN Working Group on Indigenous Populations, der UN Commission on Human Rights, ihrer Sub-commission und ihrem Special Rapporteur, dem ILO Convention Compliance Monitoring, den Verfahren unter der UN Rassendiskriminierungskonvention oder den Berichten der Inter-American Commission on Human Rights werden konkrete Fälle von indigenen Gruppen diskutiert und diese somit von den Verfassern der Berichte als Indigene anerkannt. ${ }^{491}$

Ebenso werden in den Beschwerdeverfahren im Rahmen des ersten Zusatzprotokolls des IPbpR Vertreter indigener Gemeinschaften als Beschwerdeführer anerkannt. Im Fall Ominayak, Chief of the Lubicon Lake Band v. Canada wurde bei der Würdigung der substantiellen Aspekte einer Verletzung der kulturellen Integrität der Gruppe unter Artikel 27 IPbpR eine Verletzung durch Kanada festgestellt. ${ }^{492}$ Auch wenn in diesem Fall die Existenz des Lubicon Lake Band von Kanada nicht bestritten wurde, zeigte dieser Fall aus den späten 1980er Jahren, dass indigene Gruppen sich mit Beschwerden an das Human Rights Committee wenden können, um Verletzungen der kulturellen Integrität der Gruppe vor einer internationalen Institution geltend zu machen, ohne sich dabei auf eine Anerkennung durch den Heimatstaat berufen zu müssen. .93

Auch die WTO akzeptierte 2001 einen Schriftsatz einer indigenen Gruppe im Fall der US-Provisional Anti-Dumping Measures on Imports of Certain Softwood Lumber from Canada als amicus curiae, in dem die Interior Alliance of BC Indian Nations die Beschwerde der US-Amerikaner unterstützte und dabei die Frage aufwarf, ob ,the non-recognition of Aboriginal land rights constitutes a subsidy under international

490 Organisation of African Unity, „Fourteenth Annural Activity Report of the African Commission on Human and Peoples' Rights", AHG/229(XXXVII), 2.-12. Juli 2001, Resolution on the Rights of Indigenous People/Communities in Africa, S. 14f; African Commission's Working Group of Experts on Indigenous Populations/Communities, „Report of the African Commission's Working Group of Experts on Indigenous Populations/Communities“,

DOC/OS(XXXIV)/345, 20. November 2003, S. 6ff; Siehe im übrigen auch: African Commission on Human and People's Rights, International Work Group for Indigenous Affairs, Indigenous Peoples in Africa: The forgotten Peoples?, 2006.

${ }^{491}$ Ein Überlick über die Verfahren dieser Organe findet sich bei: Anaya, Indigenous peoples in international law, S. 217-247.

492 Ebd., S. 254-256.

493 Weitere Fälle des Human Rights Committee finden sich bei: Ebd., S. 253-258; sowie bei: Raija Hanski, Leading cases of the Human Rights Committee, 2007. 
trade law“.494 Die Weltbank hat eine eigene Indigenous Peoples Policy und fördert Projekte in allen Regionen der Welt. ${ }^{495}$ Auch die Asian Development Bank verfolgt eine gesonderte Politik in Bezug auf indigene Völker. ${ }^{496}$

Die Organe der internationalen Institutionen beurteilen somit die Existenz substaatlicher indigener Gruppen, indem sie deren Vertreter sowie die Selbstidentifikation als, indigene Völker ${ }^{6}$ anerkennen. Die Praxis internationaler Organisationen zeigt somit einen Umgang mit indigenen Völkern, der eine individuelle Anerkennung von Gruppen impliziert, so dass diese ihren Status unabhängig von einer Anerkennung der Regierung ihrer Heimatstaaten international vertreten können.

\section{Anerkennung durch NGOs}

Die Anerkennung indigener Völker erfolgt auch in ihren Beziehungen untereinander, um sich in überregionalen Zusammenschlüssen politisch koordinieren und positionieren zu können. So wurde beispielsweise 1999 in Indonesien eine Vereinigung der indigenen adat Gemeinschaften unter dem Namen Aliansi Masyarakat Adat Nusantara $(A M A N)$ gegründet. $A M A N$ machte bei ihrer Gründungsdemonstration unter dem Motto „Wenn ihr uns nicht anerkennt, erkennen wir Euch auch nicht an“ ihre progressive politische Position gegenüber der indonesischen Regierung deutlich. ${ }^{497}$ Sie beriefen sich dabei unter anderem auf die UN Deklaration über die Rechte indigener Völker von 2007. Im Jahr 2010 umfasste diese Organisation 1163 adat Gemeinschaften. ${ }^{498}$ Sie gliedert sich in regionale Unterorganisationen und ist international vernetzt. Die Mitglieder von $A M A N$ Toraya nehmen an den Aktivitäten internationaler NGOs und UN Unterorganisationen für indigene Gruppen teil und mehrere adat-Oberhäupter partizipierten an sogenannten adat-Trainings des Indigenous People's Programme der UN in anderen Regionen Indonesiens, in Malaysia oder den Philippinen. ${ }^{499}$

${ }^{494}$ „WTO Members Comment on Indigenous Amicus Brief in Lumber Dispute“, International Centre for Trade and Sustainable Development, 16. Mai 2002; World Trade Organization, „United States Preliminary Determinations with Respect to Certain Softwood Lumber from Canada, Report of the Panel“", WT/DS236/R, 27. September 2002, Abs. 7.2; Interior Alliance Indigenous Nations, Brief an die: World Trade Organization, Mr. Dariusz Rosati, „United States - Preliminary Determinations with Respect to Certain Softwood Lumber from Canada, WT/DS236“, 15. April 2002; Barsh, „Indigenous Peoples“, S. 844.

495 World Bank, „Operational Manual, OP 4.10 - Indigenous Peoples“, Juli 2005, überarbeitet April 2013; Navin Rai et al., „Implementation of the World Bank's Indigenous Peoples Policy, A Learning Review (FY 2006-2008)“, OPCS Working Paper, August 2011, S. 11.

496 Asian Development Bank, „The Bank’s Policy on Indigenous Peoples“, April 1998.

${ }^{497}$ Klenke und Socha, „Emerging Indigeneity - Völkerrechtswissenschaft und ethnologische Praxis subnationaler kultureller Gemeinschaften“.

${ }^{498}$ Ebd.

${ }^{499}$ Ebd. 
Die Vernetzung indigener Gruppen in regionalen, nationalen und internationalen Gruppen in Form von NGOs bildet die Grundlage der globalen Indigenenbewegung und ihrer politischen Wirkmächtigkeit. Dabei spielten insbesondere NGOs aus den Bereichen Umweltschutz und Entwicklungszusammenarbeit eine entscheidende Rolle, die sich mit ihren jeweils eigenen Interessenschwerpunkten und Zielen mit lokalen Gruppen in Verbindung setzten und so, wie dies auch das Beispiel der Toraja im Kontext von $A M A N$ zeigt, die Diskurse um indigene Identität teilweise erst anstießen. ${ }^{500}$ Mittlerweile hat das globale Netzwerk von NGOs indigener Völker eine unüberschaubare Komplexität mit den unterschiedlichsten Formen und Voraussetzungen der Anerkennung neuer Organisationen und Gruppierungen entwickelt.

\section{Zusammenfassung: international gefestigte Positionen und geregelte Verfahren für neue indigene Gruppen}

Die vorangegangene Darstellung einiger Beispiele der Anerkennung indigener Völker zeigt, dass sich im Zuge der globalen Indigenenbewegung feste Strukturen entwickelt haben, in denen indigene Völker anerkannt wurden und in denen bis heute neue Gruppen um Anerkennung als Indigene ringen. Angesichts dieser andauernden Dynamik haben sich auf nationaler Ebene teilweise gefestigte Institutionen entwickelt, bei denen konkrete Vorgaben für Voraussetzungen und zulässige Beweismittel des Anerkennungsprozesses festgelegt wurden. Es zeigt sich auch, dass trotz der globalen Vernetzung von NGOs und der gefestigten Position indigener Institutionen in internationalen Organisationen der Zugang zur Teilnahme an Staatenkonferenzen immer noch von einer formellen $\mathrm{Zu}$ stimmung und Anerkennung der Staaten abhängt. Vor dem Hintergrund der etablierten Rolle indigener Vertreter und ihres politischen Gewichts auf internationaler Ebene ist Widerspruch und Nicht-Anerkennung in der Praxis der Zulassungsverfahren zum Beobachterstatus bei Staatenkonferenzen nicht zu erwarten.

\section{Lokale Gemeinschaften}

Die Anerkennung lokaler Gemeinschaften im Kontext des internationalen Umweltrechts vollzieht sich ebenfalls auf den verschiedenen Ebenen nationaler und internationaler Institutionen. Aus der engen Verknüpfung zu Politiken indigener Völker ergeben sich verschiedene Überlagerungen, bei denen die Anerkennung indigener und lokaler Gemeinschaften institutionell und auch in ihren Begrifflichkeiten nicht zu differenzieren sind. Für eine Bewertung der rechtlichen Bedeutung der Anerkennung ist eine genaue Zuordnung der folgenden Beispiele

${ }^{500}$ Ebd. 
von Anerkennungsverfahren im Umweltvölkerrecht allerdings auch nicht zwingend notwendig.

\section{Anerkennung durch Staaten}

Für die Anerkennung lokaler Gemeinschaften von staatlicher Seite ist die Ausgestaltung nationaler Regelungen bei der Implementierung internationaler Vorgaben maßgeblich. So hängt die Einbeziehung und Anerkennung lokaler Gemeinschaften einerseits davon ab, wie auf nationaler Ebene entsprechende Definitionen und Verfahren ausgestaltet werden und andererseits, wie etwaige Ermessensspielräume bei ihrer Anwendung genutzt werden. Der gesetzliche Rahmen auf nationaler Ebene bestimmt somit die Position lokaler Gemeinschaften gegenüber staatlichen Behörden und multinationalen Unternehmen bezüglich ihres prior informed consent zum bioprospecting und ihrer Beteiligung am access and benefit sharing.

Die Anerkennung lokaler Gemeinschaften zeigt sich auf internationaler Ebene in den Staatenberichten zur Umsetzung der CBD und in den regional reports im Rahmen der working group on Article 8(j). ${ }^{501}$ Darin werden allgemein die Maßnahmen auf nationaler Ebene vorgestellt und konkrete Fälle lokaler Gemeinschaften angesprochen. Es zeigte sich hier aber auch die grundsätzliche Nicht-Anwendung des Konzepts ,lokaler Gemeinschaft‘ im Regionalbericht für Nordamerika.

While Article 8(j) also makes reference to local communities, no definition has been offered by the Conference of the Parties as to what constitutes a 'local community' and it is uncertain whether any community in North America would qualify. ${ }^{502}$

In Kanada und den USA werden die Regelungen des Artikel 8(j) CBD somit nur über das Konzept der indigenen Gemeinschaft umgesetzt.

Eine Anerkennung indigener und lokaler Gemeinschaften auf nationaler Ebene wird auch mit den Datenbanken zu traditionellem Wissen umgesetzt, die zum Schutz gegen Biopiraterie bestehendes Wissen sammeln und so gegen eine Patentierung als neue Erfindungen schützen sollen. Solche staatlichen Datenbanken finden sich beispielsweise in Indien ${ }^{503}$, Brasilien ${ }^{504}$ oder Peru ${ }^{505}$. Darin werden

501 Beispielhaft: Ad hoc open ended working group on Article 8(j) and Related Provisions of the Convention on Biological Diversity, „Composite report on the status and trends regarding the knowledge, innovations and practices of indigenous and local communities - Regional Report: Central America“, UNEP/CBD/WG8J/3/INF/6, 30. September 2003.

502 Ad hoc open ended working group on Article 8(j) and Related Provisions of the Convention on Biological Diversity, „Composite report on the status and trends regarding the knowledge, innovations and practices of indigenous and local communities - Regional report: North America“, UNEP/CBD/WG8J/3/INF/8, S. 4.

${ }^{503}$ Council of Scientific and Industrial Research, „Traditional Knowledge Digital Library“. 
traditionelle Praxen und Wissen gesammelt und bestimmten Gruppen zugeordnet, was die Anerkennung dieser Gruppen als lokale Gemeinschaften von staatlicher Seite impliziert.

\section{Anerkennung durch internationale Organisationen}

Auf internationaler Ebene werden lokale Gemeinschaften in den Kontexten internationaler Umweltschutzstandards anerkannt. So erteilt die internationale Walfangkommission einzelne Fanglizenzen für indigene Gemeinschaften, die in Fortführung ihrer historisch bestehenden Praxen auf den Walfang angewiesen sind, um ihre notwendige Versorgung mit Nahrung zu gewährleisten. Auf Anfrage der nationalen Regierungen, welche die kulturellen Hintergründe und subsistenzwirtschaftliche Notwendigkeit einer Erlaubnis für aboriginal subsistence whaling vorlegen müssen, entscheidet ein scientific committee über die Fangquoten unter Berücksichtigung der Maßstäbe zur Arterhaltung. ${ }^{506}$ In den Anträgen der Staaten werden auf Grundlage anthropologischer Studien und historischer Quellen die kulturellen Praxen und heutige wirtschaftliche Notwendigkeit des Walfangs für indigene Gruppen dargelegt. So beginnt beispielsweise die Beschreibung des historischen Hintergrundes des russischen Antrags für 2003-2007 folgendermaßen:

The aboriginal people of Chukotka, Asiatic Eskimos and the Marine Chukchi, have harvested whales since 'time immemorial' (Ainana et al., 1999; Ainana et al., 1997; Zelensky et al., 1997). Whale harvesting was one of the main activities that allowed the ancestors of today's Chukotka aboriginal residents to settle on the coast of the northeastern part of the Asian continent (Krupnik, 1994; Krupnik, 1987). Specialists can demonstrate that aboriginal harvesting of whales is at least 2,000 years old in Chukotka from the archeological record (Savinetsky, 2001; Krupnik, 1994; Bogoslovskaya et al., 1984; Krupnik, 1987; Zemsky and Bogoslovskaya, 1997a; Zemsky and Bogoslovskaya, 1997b). ${ }^{507}$

\footnotetext{
${ }^{504}$ Instituto do Patrimônio Histórico e Artístico Nacional, „Banco de Dados de Bens Culturais Imateriais Registrados".

505 Instituto Nacional de Defensa de la Competencia y de la Protección de la Propiedad Intelectual „Registro de Conocimientos Colectivos de Pueblos“.

506 Alexander Gillespie, „Aboriginal Subsistence Whaling: A Critique of the Inter-Relationship Between International Law and the International Whaling Commission", Colorado Jounral of International Environmental Law and Policy Vol. 12 (2001), S. 83ff; International Whaling Comission, „Aboriginal subsistence whaling“.

${ }^{507}$ R.G. Borodin et al., „Cultural, Traditional and Nutritional Needs of the Aboriginal Population of Chukotka for Gray Whales and Bowhead Whales 2003-2007““, IWC/54/AS/5, 2002, S. 6.
} 
Die Fangquoten werden daraufhin gegliedert nach Spezies, Region und Zeitraum vom scientific committee festgelegt. ${ }^{508}$ Insgesamt wurden so im Jahr 2010 von indigenen Gruppen aus Dänemark, Russland, St. Vincent und den Grenadienen sowie den USA, also den vier Staaten, in denen aboriginal subsistence whaling gestattet ist, ca. 400 Wale gefangen. ${ }^{509}$ Im speziellen Kontext des Walfangs müssen also die Regierungen mit konkreten Anträgen die kulturelle Praxis des Walfangs indigener Gruppen sowie die wirtschaftliche Notwendigkeit belegen, um vom scientific committee der International Whaling Commission gesonderte Fangquoten gestattet zu bekommen. Dieses Antragsverfahren bildet somit den Rahmen für die Zusammenarbeit von indigenen Gemeinschaften und den Regierungen ihrer Heimatstaaten sowie für die Anerkennung dieser Gruppen durch die International Whaling Commission.

Eine Anerkennung indigener und lokaler Gemeinschaften durch internationale Organisationen erfolgt auch, wenn Vertreter von NGOs im Namen ihrer Gruppen an internationalen Verhandlungen teilnehmen, wie dies im Kontext des internationalen Umweltrechts zunehmend der Fall ist. Im IGC der WIPO haben im Rahmen der Beteiligung von NGOs eine Vielzahl von Vertretern indigener und lokaler Gemeinschaften Anerkennung gefunden. ${ }^{510}$ Zur Teilnahme ist eine Akkreditierung notwendig, welche durch eine Entscheidung der Mitgliedstaaten bestätigt wird. Die Akkreditierung wird über ein Standardformular beim Sekretariat beantragt, in welchem der Name, eine Beschreibung, die Ziele und Aktivitäten sowie der hauptsächliche Wirkungsort angegeben werden. Bisher wurden alle Anträge von Seiten der Mitgliedstaaten einstimmig angenommen, so dass mittlerweile über 200 Organisationen als Beobachter zugelassen sind. ${ }^{511}$

Zur finanziellen Unterstützung der Teilnahme von Vertretern von indigenen und lokalen Gemeinschaften wurde in der sechsten Sitzung des IGC der Voluntary Fund eingerichtet. ${ }^{512}$ Eine der Voraussetzungen der Förderung aus diesem Fonds ist die Zugehörigkeit zu einer Organisation, welche ,indigenous and local communities, and other customary holders or custodians of traditional knowledge and

\footnotetext{
508 Alexander Gillespie, „Aboriginal Subsistence Whaling: A Critique of the Inter-Relationship Between International Law and the International Whaling Commission", Colorado Jounral of International Environmental Law and Policy Vol. 12 (2001), S. 83ff; International Whaling Comission, „Aboriginal subsistence whaling“".

509 International Whaling Comission, „Aboriginal Subsistence Whaling Catches Taken“.

510 Stefan Groth, Negotiating Tradition - The Pragmatics of International Deliberations on Cultural Property, 2011, S. 50.

511 WIPO IGC, „Accredited observers to the WIPO Intergovernmental Committee on Intellectual Property and Genetic Resources, Traditional Knowledge and Folklore (IGC)“.

512 WIPO Generalversammlung, „Establishment of the WIPO Voluntary Fund for Accredited Indigenous and Local Communities", Annex zu WO/GA/32/6, 24. August 2005, geändert durch WO/GA/39/11, 5. Juni 2010.
} 
traditional cultural expressions“ repräsentiert. 513 Die Entscheidung dazu trifft das Advisory Board welches aus neun Mitgliedern besteht:

the Chair of the Committee, appointed ex officio or, where that proves to be impossible, one of the Vice-Chairs nominated by the Chair as his or her deputy;

five members from the delegations of WIPO Member States taking part in the Committee, reflecting an appropriate geographical balance; and

three members from accredited observers representing indigenous and local communities or other customary holders or custodians of TK or TCEs. ${ }^{514}$

Entscheidungen über die finanzielle Unterstützung bedürfen einer Mehrheit von sieben Stimmen. Ein Antrag ist endgültig abgelehnt, wenn er nur drei oder weniger Stimmen erhält, so dass die Mitgliedstaaten zusammen mit dem Chair solch eine Entscheidung durchsetzen könnten. ${ }^{515}$ Die Entscheidung zur Förderung durch den Voluntary Fund impliziert die Anerkennung als Repräsentant einer indigenen und lokalen Gemeinschaft und wird im Advisory Board auf konkret-individueller Basis beschieden.

Der formalisierte Prozess zur Beantragung des Beobachterstatus' und auch der Unterstützung durch den Voluntary Fund setzt allerdings voraus, dass sich die Gruppen ihrer eigenen Rolle als indigene und lokale Gemeinschaft bewusst sind und die notwendigen Kompetenzen und Mittel aufweisen, um den Status wirklich beantragen und Vertreter entsenden zu können. Der individuelle Prozess der Akkreditierung wirkt dabei auch auf die Gruppe selbst zurück, indem sie ihre Rolle im globalen Kontext der verhandelten Themen verorten und diese Position auch gegenüber lokalen Akteuren oder auch der eigenen Regierung gegenüber vertreten muss. ${ }^{516}$

\section{Zusammenfassung: Anerkennung im Rahmen umweltrechtlicher Politiken}

Die Anerkennung indigener und lokaler Gemeinschaften im Umweltvölkerrecht erfolgt vorrangig auf nationaler Ebene im Zuge der Umsetzung umweltrechtlicher Instrumente. Dabei obliegt die Ausgestaltung und Anwendung der Anerkennungsverfahren den staatlichen Akteuren und kann nur über den nationalen politischen Diskurs von den lokalen Gemeinschaften beeinflusst werden. Auch für Sonderregelungen zu kulturellen Praxen im Umweltrecht haben die Staaten die Entscheidungshoheit der Anerkennung indigener und lokaler Gemeinschaf-

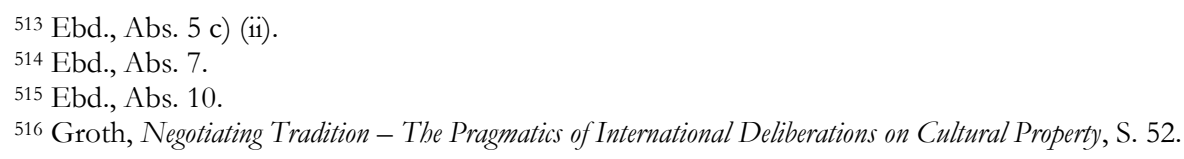


ten inne. Das Beispiel der internationalen Walfangkommission zeigt, dass die Antragskompetenz für solche Sonderregelungen bei den Heimatstaaten liegt, welche einen entsprechenden Antrag stellen und dafür die Hintergründe und Notwendigkeiten für Ausnahmen von Artenschutzstandards für indigene Völker eingehend begründen müssen. Und auch für die Beteiligung von Vertretern indigener und lokaler Gemeinschaften bei internationalen Verhandlungen, die eine Anerkennung ihrer Gruppen impliziert, ist die Zustimmung der Staaten notwendig.

\section{Akteurskonstellationen der Anerkennung}

Die Konstellationen der beteiligten Akteure bei der Anerkennung von substaatlichen Gruppen gehen über die einfache Gegenüberstellung von diesen mit ihrem jeweiligen Heimatstaat hinaus. Im konkreten Kontext der unterschiedlichen Konzepte ergeben sich verschiedene Beziehungen zwischen den Gruppen und ihrem Heimatstaat, Drittstaaten, internationalen Organisationen und NGOs, bei denen jeweils Fragen der Anerkennung aufgeworfen und unterschiedlich behandelt werden.

\section{Staatliche Anerkennung}

Die meisten Instrumente, die Regelungen für substaatliche Gruppen treffen, normieren das Verhältnis zu ihrem Heimatstaat. ${ }^{517}$ Dies zeigt sich beispielsweise in den Rechten von Minderheiten zum Gebrauch ihrer Sprache im behördlichen Verkehr sowie der Übertragung von staatlichen Regelungskompetenzen auf indigene Völker. Auch die Mitbestimmungsrechte indigener und lokaler Gemeinschaften zum bioprospecting genetischer Ressourcen und ihres assoziierten traditionellen Wissens werden jeweils durch die Staaten geregelt. In der Regel ist es somit der Heimatstaat, der verpflichtet ist, substaatliche Gruppen anzuerkennen, ihnen gewisse Rechte einzuräumen und diese zu gewährleisten. Die Anerkennung und Nicht-Anerkennung von ,Minderheiten', ,indigenen Völkern` oder ,lokalen Gemeinschaften'von Seiten des Staates, in dem die Gruppe lebt, gestaltet sich jedoch jeweils unterschiedlich.

So berufen sich Staaten teilweise kategorisch auf eine grundsätzliche Nichtanwendbarkeit bestimmter Konzepte oder formelle Ausschlussgründe. Dies zeigt insbesondere das Argument der Unanwendbarkeit des Konzepts der Indigenität in Afrika und Asien, wo das Merkmal der Verbindung zu präkolonialen Gesellschaften keine sinnvolle Unterscheidung liefere und somit grundsätzlich abgelehnt

517 Siehe dazu die Ausführungen unter „A. III. 4. Umsetzung des internationalen Minderheitenschutzes“ auf S. 25, „B. III. Das Selbstbestimmungsrecht“ auf S. 50, „C. III. Regelungen zum Schutz traditionellen Wissen“" auf S. 76 sowie „C. IV. Nationalgesetzlicher Rahmen und Rechte geistigen Eigentums“" auf S. 78. 
wird. Ebenso erkannten einige südamerikanische Staaten die Anwendbarkeit des internationalen Minderheitenschutzes in ihren Ländern nicht an, da ihrer Ansicht nach Minderheiten nur solche Gruppen sind, die sich ,en bloc' und nicht freiwillig als Minderheit in einem Staat befinden. ${ }^{518}$ Migranten und Siedler, die in einem freiwilligen Wanderungsprozess eine kulturell distinkte Gruppe in einem Staat gebildet haben, fielen dieser Argumentation folgend nicht unter den internationalen Minderheitenschutz. ${ }^{519}$ Ein weiteres Beispiel ist die grundsätzliche NichtAnerkennung ,lokaler Gemeinschaften' in Nordamerika, wo mit dem Argument einer mangelnden konkreten Definition dieses Konzepts seine Anwendung insgesamt abgelehnt wird. Stattdessen werden hier die umweltrechtlichen Regelungen nur auf indigene Völker bezogen, die allerdings bereits eine etablierte Position innehaben und entsprechend in den Regelungsbereich fallen. Des Weiteren wird das Nichtvorliegen der Staatsbürgerschaft für Teile der Gruppe oder einzelne Individuen als formeller Ausschlussgrund von staatlicher Seite zur Anwendung des Minderheitenschutzes in Europa angeführt.

Schließlich ergeben sich jeweils unterschiedliche Prozesse der Anerkennung für einzelne Gruppen, wenn sie sich im Sinne eines der Konzepte identifizieren. Halten die Regierungen eines oder mehrere Konzepte in ihren Territorien für anwendbar, nehmen die Staaten eine Schlüsselrolle sowohl auf nationaler als auch internationaler Ebene ein, wenn es um die individuelle Anerkennung substaatlicher Gruppen geht. Die Anwendung etwaiger Minderheiten- oder Selbstbestimmungsrechte, die Einbeziehung lokaler Gemeinschaften bei Entscheidungen zum Zugang zu genetischen Ressourcen oder die Beteiligung von Nichtregierungsorganisationen indigener und lokaler Gemeinschaften bei internationalen Verhandlungen hängt - dies haben die Beispiele gezeigt - in den meisten Fällen von der Genehmigung ihrer Heimatstaaten ab. Diese Genehmigung impliziert dann die Anerkennung einzelner Gruppen im Sinne eines der drei Konzepte, die sich in unterschiedlichen Verfahren zeigt: Es sind die Regierungen, Gerichte und Behörden, welche die Anerkennung substaatlicher Gruppen im Sinne eines der Konzepte aussprechen. Teilweise etablierten sich gefestigte institutionelle Strukturen zur Anerkennung, wie dies das Beispiel des Bureau of Indian Affairs zur Anerkennung indigener Völker in den USA zeigt. Aufgrund der politischen Bedeutung der Anerkennung substaatlicher Gruppen liegt die Entscheidungskompetenz jedoch meist bei der Exekutive.

518 Thornberry, International law and the rights of minorities, S. $154 \mathrm{f}$.

519 Ebd. 


\section{Anerkennung durch Drittstaaten}

Die Anerkennung von substaatlichen Gruppen durch Drittstaaten zeigt sich explizit bei der Anbahnung zum Abschluss bilateraler Verträge. Bilaterale Minderheitenschutzverträge werden zwischen den Heimatstaaten und den Staaten geschlossen, mit denen die Minderheit ihre kulturellen Wurzeln teilt. Diese ,kulturellen Heimatstaaten' der Minderheit identifizieren substaatliche Gruppen als ,Minderheiten' und beeinflussen über das Vertragsschlussverfahren die Anerkennung der Minderheit durch den Heimatstaat.

Die Anerkennung von substaatlichen Gruppen durch Drittstaaten zeigt sich auch in grenzüberschreitenden politischen Diskursen. So rief beispielsweise das französische Gesetz zum Verbot der Leugnung des Völkermordes an den Armeniern in der Türkei, welches einen entsprechenden Status der Armenier impliziert, heftige Proteste von Seiten der türkischen Regierung hervor. ${ }^{520}$ Problematisch ist dabei die Grenze des völkerrechtlichen Nichteinmischungsgebots, welches Einflussnahmen auf dem Territorium anderer Staaten verbietet.

\section{Internationalisierte Anerkennung}

Substaatliche Gruppen sind im Rahmen internationaler Organisationen nicht nur passives Objekt und Thema in etwaigen Verhandlungen. Vielmehr beteiligen sie sich aktiv als Teilnehmer an Staatenkonferenzen, treten im Rahmen von Beschwerde- und Berichtsverfahren vor internationalen Institutionen auf und interagieren mit internationalen Organisationen als Partner bei der Umsetzung von Projekten.

Insbesondere Vertreter indigener Völker sind seit den 1970er Jahren auf internationaler Ebene präsent. Sie nehmen als Beobachter an Staatenverhandlungen teil und organisieren sich unabhängig von staatlichem Einfluss im Rahmen internationaler Organisationen. Diese Einbeziehung indigener Gruppen erfolgt im Zuge der Akkreditierung als Vertreter zivilgesellschaftlicher Interessenvertretung und impliziert gleichzeitig die Anerkennung als ,indigenes Volk' sofern dies aus einer Selbstidentifikation im Registrierungsverfahren hervorgeht. Die Entscheidungsstrukturen zur Anerkennung sind in den verschiedenen Organisationen unterschiedlich ausgestaltet: Teilweise trägt das Plenum der Mitgliedstaaten die Entscheidung, teilweise eine repräsentative Gruppe, teilweise ein gesondertes Gremium unterschiedlicher Zusammensetzung. Bei der Anerkennung durch das UNPFII sind gar keine Staatenvertreter beteiligt. Die Anerkennung als Vertreter eines indigenen Volkes zu Verhandlungen in internationalen Organisationen bedeutet jedoch nicht, dass gleichzeitig entsprechende Rechte für diese Gruppe auf nationaler Ebene gewährt werden. Sie ist vielmehr auf den spezifischen Kontext der Teilnahme in einem bestimmten Forum beschränkt. Gleichzeitig wirkt sie

520 „France-Turkey Armenia ,genocide“ row reflected in media“, BBC, 24. Januar 2012. 
jedoch als Argument auf die politische oder rechtliche Aushandlung des Gruppenstatus' auf nationaler Ebene zurück.

Diese Relativität der Anerkennung bildet ebenfalls den Rahmen der Anerkennung substaatlicher Gruppen bei internationalen Beschwerdeverfahren. Das Human Rights Committee nimmt Beschwerden substaatlicher Gruppen im Rahmen des Individualbeschwerdeverfahrens unter dem ersten Zusatzprotokoll entgegen und erkennt diese somit unter Artikel $27 \mathrm{IPbpR}$ als Minderheiten oder indigene Völker an. Diese Anerkennung beschränkt sich jedoch auf das Verhältnis der substaatlichen Gruppe zum Human Rights Committee bzw. zur jeweils angerufenen internationalen quasi-gerichtlichen Institution. Die Entscheidung und die Umsetzung durch den Heimatstaat wirken dann als Argument im Aushandlungsprozess des Gruppenstatus auf nationaler Ebene.

Eine vorausgehende Anerkennung einer substaatlichen Gruppe als ,Minderheit ${ }^{\star}$ durch den Heimatstaat ist keine notwendige Voraussetzung für die Beschwerdebefugnis vor einer internationalen Beschwerdeinstitution. Bereits die Studie von Francesco Capotorti proklamierte, dass der internationale Schutz von Minderheiten nicht von der offiziellen staatlichen Anerkennung ihrer Existenz abhängen könne. ${ }^{521}$ Dennoch besteht kein Automatismus, der die Anerkennung einer Gruppe durch internationale Institutionen auch auf nationaler Ebene rechtsverbindlich macht. Der Heimatstaat ist nur zur Umsetzung einer internationalrechtlichen Entscheidung und nicht zu einer darüber hinausgehenden Anerkennung eines Rechtsstatus' der Gruppe verpflichtet. Die politische Wirkmächtigkeit einer Entscheidung internationaler Institutionen wird eine Anerkennung in der Regel jedoch unumgänglich machen.

Für die Anerkennung substaatlicher Gruppen bei Berichtsverfahren internationaler Institutionen, wie sie beispielsweise in der Framework Convention oder in der $C B D$ vorgesehen sind, ist zwischen Staatenberichten und Berichten unabhängiger Gremien der internationalen Organisationen zu unterscheiden. Individuelle Staatenberichte zur Situation substaatlicher Gruppen, die von Seiten der Regierungen für internationale reports verfasst werden, bedeuten eine Anerkennung dieser Gruppen auch auf nationaler Ebene. Berichte, die von unabhängigen internationalen Gremien über substaatliche Gruppen erarbeitet werden, erklären eine Anerkennung dieser Gruppe nur im Verhältnis zu der Institution, die den Bericht verfasst hat. Die Zurückweisung des Berichts des Beratenden Ausschusses der Framework Convention von Seiten Deutschlands zur Situation der Sinti und Roma ohne deutsche Staatsangehörigkeit zeigt beispielhaft die Relativität der Anerkennung substaatlicher Gruppen vis à vis internationaler Institutionen. Deutschland

521 Sub-Commission on the Prevention of Discrimination and Protection of Minorities, Francesco Capotorti, ,Study on the rights of persons belonging to ethnic, religious, and linguistic minorities“, UN Doc. E/CN.4/Sub.2/384/Rev.1, Add. 1, Abs. 41; Thornberry, International law and the rights of minorities, S. 157. 
gewährt dieser Gruppe keinen Minderheitenstatus. Der Beratende Ausschuss befasst sich aber dennoch mir ihrer Situation unter Gesichtspunkten des Minderheitenschutzes. Eine Harmonisierung dieser sich widersprechenden Auffassungen kann nur über den politischen Diskurs erreicht werden, der eine Anerkennung der nicht-deutschen Sinti und Roma als Minderheit von Seiten Deutschlands zur Folge haben kann.

Die Relativität der Anerkennung substaatlicher Gruppen zeigt sich außerdem bei der Zusammenarbeit internationaler und regionaler Organisationen mit substaatlichen Gruppen. Die Weltbank befasst sich beispielsweise in Berichten und Projekten ihrer Indigenous Peoples Policy auch mit den Staaten, in denen die Regierungen die Anwendbarkeit dieses Konzepts kategorisch ausschließen. ${ }^{222}$ Die internationalisierte Anerkennung substaatlicher Gruppen beeinflusst in dieser Form den nationalen politischen Diskurs zur Anerkennung von Minderheiten, indigenen Völkern oder indigenen und lokalen Gemeinschaften.

Zusammenfassend lässt sich feststellen, dass die Anerkennung substaatlicher Gruppen im Sinne eines der drei Konzepte auf internationaler Ebene zumeist unabhängig von einer Zustimmung des Heimatstaates der Gruppe erfolgt. Sie ist jedoch auf die unmittelbare Beziehung zwischen der Gruppe und der internationalen Institution beschränkt. Ebenso ergeben sich inhaltliche Grenzen der Anerkennung auf den funktionellen Kontext der Teilnahme an internationalen Verhandlungen, als Beschwerdeführer vor einer quasi-gerichtlichen Instanz oder bei der Umsetzung konkreter Entwicklungsprojekte. Die Anerkennung als ,Minderheit', ,indigenes Volk ${ }^{6}$ oder , lokale Gemeinschaft ${ }^{6}$ durch internationale Akteure wirkt jedoch gleichzeitig auf den politischen Diskurs zur Anerkennung des Rechtsstatus' der Gruppe auf nationaler Ebene zurück.

522 Dies zeigen die Berichte zur Situation indigener Völker in der Zentral afrikanischen Republik, dem Kongo, Gabon, Indien, China, der Volksrepublik Laos und Vietnam. Prospere

Backiny-Yetna, Quentin Wodon, „Census-based profile of the Mbororos and the Pygmies in the Central African Republic“, World Bank - Central African Republic Country Brief No. 8, 1. Mai 2011; Arbi Ben-Achour, Propere Backiny-Yetna und Quentin Wodon, „Socioeconomic status of the Pygmies in the Democratic Republic of Congo", World Bank - Democratic Republic of Congo Country Brief No. 6, 1. April 2011; Propspere Backiny-Yetna und Quentin Wodon, „Census-based profile of the Pygmies in Gabon“, World Bank - Gabon Country Brief No. 5, 1. März 2011; Maitreyi Bordia Das, Gillette Hall, Soumya Kapoor und Denis Nikitin, „Indias's Adivasis“, World Bank - India Country Brief No. 4, 1. Januar 2011; Emily Hannum, „Ethnic disparities in China: geography, rurality, and socioeconomic welfare", World Bank - China Country Brief No. 3, 1. Januar 2011; Elizabeth M. King und Dominique von de Walle, „Catching up slowly: ethnic and gender inequalities in Lao PDR“, World Bank - Lao PDR Country Brief No. 2, 1. November 2010; Hai-Anh H. Dang, „Growth in Vietnam is strong but not shared equitably across ethnic groups“, World Bank - Vietnam Country Brief No. 1, 1. September 2009; Gillette Hall, Harry A. Patrinos, Indigenous Peoples, Poverty, and Development, 2014. 


\section{Nichtstaatliche Anerkennung}

Politisch relevant sind auch Anerkennungspraxen internationaler NGOs, die Gruppen als Minderheiten, Indigene oder lokale Gemeinschaften anerkennen. Es findet sich eine vielfältige Landschaft von Organisationen, in denen sich indigene Gruppen organisieren, austauschen und politisch Einfluss nehmen. Auch im Verhältnis zu multinationalen Konzernen, zu denen indigene Völker insbesondere in Auseinandersetzungen um Bodenschätze vielfach in Konflikte geraten sind, stellen sich Fragen der Anerkennung, die über das bilaterale Verhältnis der beteiligten Streitparteien hinaus gehen und somit weiteren politischen Einfluss haben. ${ }^{523}$

Wissenschaftliche Studien von Anthropologen und Ethnologen haben ebenfalls einen Einfluss auf politische Debatten über indigene Völker, Minderheiten und lokale Gemeinschaften. Zahlreiche Publikationen über Feldforschung und Länderberichte geben Anstoß zur Selbstidentifikation von Gruppen als Indigene oder nehmen Einfluss auf nationale Diskurse um die Anerkennung von Gruppen. Außerdem werden historische und anthropologische Studien von Wissenschaftlern als Argument in Anerkennungsprozessen herangezogen und spielen so eine unmittelbare Rolle als Beweismittel in förmlichen Anerkennungsverfahren.

\section{Zusammenfassung: eine Perspektive der Anerkennung}

Die Darstellung, wie die Konzepte substaatlicher Gruppen im Einzelfall zur Anwendung kommen, zeigt eine vielfältige Landschaft von beteiligten Akteuren, unterschiedlicher Verfahren und Entscheidungsmomenten. Mangels völkerrechtlicher Vorgaben entwickelten sich viele unterschiedliche Umsetzungsverfahren, die auf verschiedenen Ebenen, in unterschiedlichen Prozessen, mit je eigenen Graden an Formalisierung und unter je eigenen Voraussetzungen für die Anwendung der Rechte von Minderheiten, indigenen Völkern und lokalen Gemeinschaften am Werk sind. Teilweise ergeben sich für eine Gruppe in unterschiedlichen Kontexten gegenüber unterschiedlichen Institutionen im Ergebnis auch divergierende Entscheidungen über ihren Rechtsstatus. Alle Verfahren der Umsetzung der drei Konzepte in der Praxis weltweit zu untersuchen, bedürfte einer eigenen, umfassenden Studie. Die Beispiele hier zeigen allerdings die Bandbreite an unterschiedlichen Möglichkeiten, wie der Rechtsstatus substaatlicher Gruppen entsteht.

Mangels global konsentierter Definitionen, welche die Umsetzung der Konzepte substaatlicher Gruppen in Form von abstrakt-generellen Regelungen ver-

523 Beispiele dazu finden sich in: Benedict Kingsbury und Kirsty Gover (Hrsg.), Special Issue of the International Journal on Minority and Group Rights: Indigenous Groups and the reviews of Recognition in Asia: Cases from Japan, Taiwan, West Papua, Bali, the People's Republic of China and Gilgit, Vol. 11, No. $1 / 2$ (2004). 
rechtlichen würden, ist es bisher eine politische Entscheidung wie und ob eine substaatliche Gruppe als ,Minderheit', ,indigenes Volk' oder ,lokale Gemeinschaft anerkannt wird. Die obige Darstellung richtete ihren Fokus genau auf diesen Prozess der Anwendung der Konzepte, der als Anerkennung hier in seiner rechtlichen Bedeutung dargestellt wurde. Betrachtet man die Anwendung der Konzepte aus dieser Perspektive der Anerkennung, öffnen sich Problemfelder jenseits der Definitionendebatten, die rechtlich relevant und greifbar sind.

Rückt man die Anerkennung, also den Prozess der Umsetzung der Konzepte, in den Fokus, so lassen sich beteiligte Akteure, Verfahren, jeweilige Voraussetzungen und Formalia darstellen sowie gegebene Entscheidungskompetenzen, Verantwortlichkeiten, finanzielle und zeitliche Implikationen, Formen der Beteiligung und Repräsentation und möglich rechtliche Verknüpfungen zwischen unterschiedlichen Akteuren analysieren. Dabei hat die vorangegangene Darstellung zunächst gezeigt, dass es mangels völkerrechtlicher Vorgaben eine riesige Bandbreite von verschiedenen Anerkennungsprozessen gibt. Beteiligt sind auf unterschiedlichen Ebenen die Legislative, die Judikative und zumeist die Exekutive. Die Dauer der Verfahren kann sich über mehrere Jahrzehnte hinziehen. Der finanzielle Rahmen ist fast nie abzusehen. Weder für die betroffene substaatliche Gruppe noch für die jeweils andere Gruppe der Mehrheit oder weitere Gruppen im Heimatstaat sind Vorgaben für die Formen der Beteiligung oder Repräsentation gemacht. Genauso wenig finden sich Regelungen, ob und wie der Rechtsstatus einer Gruppe gegenüber einer Institution möglicherweise auf ihren Status gegenüber anderen Akteuren oder Institutionen Auswirkungen haben kann.

Gleichzeitig hat die obige Darstellung gezeigt, dass am Ende der Anerkennungsverfahren eine Entscheidung steht, die als Rechtsakt in Form von Verwaltungs- oder Gerichtseinscheidungen oder als Gesetz die Anerkennung der Gruppe als ,Minderheit', indigenes Volk' oder ,lokale Gemeinschaft ${ }^{6}$ erteilt. Diese Anerkennung hat rechtlichen Gehalt, da sie den Rechtsstatus der substaatlichen Gruppe seitens des Anerkennenden formell beschließt und für die Zukunft festlegt. Die Perspektive der Anerkennung offenbart somit insbesondere die Anwendung der Konzepte substaatlicher Gruppen als Rechtsakt, der am Ende eines Verfahrens steht, in dem sich substaatliche Gruppen bezüglich ihres Rechtsstatus’ erklären.

Zusammenfassend lässt sich somit festhalten, dass eine Perspektive der Anerkennung den Blick auf Problemfelder öffnet, die in der bisherigen Definitionsdebatte keine Beachtung gefunden haben, welche die Anwendung der Konzepte substaatlicher Gruppen allerdings verrechtlichen können. Anerkennung bezeichnet dabei sowohl den Prozess als auch den Rechtsakt, die einer substaatlichen Gruppe ihren Status als ,Minderheit', indigenes Volk' oder, lokale Gemeinschaft einräumen. Mangels anwendbarer Definitionen kommt aus völkerrechtlicher Sicht der Anerkennung substaatlicher Gruppen somit die entscheidende rechtliche Wirkung über ihren Rechtsstatus zu. 


\section{B. Rechtliche Bewertung der Anerkennung substaatlicher Gruppen}

Für eine rechtliche Bewertung der Wirkung einer Anerkennung substaatlicher Gruppen mangelt es auf internationaler Ebene an positivrechtlichen Normen, die als Anknüpfungspunkt dienen könnten. Die Diskussionen um Definitionsansätze haben bisher keinerlei rechtliche Gesichtspunkte für Anerkennungsverfahren substaatlicher Gruppen umfasst. Debatten um die rechtliche Wirkung von Anerkennung finden sich im Völkerrecht jedoch bereits bei den Fragen der Entstehung von Staaten. Die sogenannte,Great Debate zu diesem Thema teilt sich in zwei Lager: Die Anerkennung sei eine zwingend notwendige Voraussetzung bei der Entstehung neuer Staaten, vertreten die Anhänger der konstitutiven Theorie; Staaten entstehen und existieren unabhängig von der Anerkennung durch andere Völkerrechtssubjekte, postulieren die Vertreter der deklaratorischen Theorie. ${ }^{524}$

Für die konstitutive Theorie wird argumentiert, dass der Rechtsstatus als Völkerrechtssubjekt nicht automatisch gewährt werden kann, da solch eine Feststellung ein komplexes Zusammenspiel von rechtlichen Fragen und Tatsachen sei; eine Aufgabe, die von irgendjemandem vollzogen werden muss. ${ }^{525} \mathrm{Im}$ Sinne eines positivistischen Völkerrechts ist Konsens die Grundlage der internationalen Rechtsordnung, so dass auch die Aufnahme neuer Rechtssubjekte der Zustimmung von Seiten der bereits bestehenden Rechtssubjekte bedarf.526 Dem wird entgegengehalten, dass die internationale Rechtsordnung zu einem bloßen Austausch von Meinungen reduziert werden würde, sollten einzelne Staaten den rechtlichen Status von bestimmten staatsähnlichen Gebilden durch entsprechende Erklärungen bestimmen können. ${ }^{527}$ Ein diverses Feld unterschiedlicher Meinungen zur Anerkennung und Nicht-Anerkennung würde zu Rechtsunsicherheit und einer Entwertung der rechtlichen vis á vis der politischen Struktur der internationalen Gemeinschaft führen. Außerdem wird gegen die konstitutive Theorie vorgebracht, sie liefere keine tragfähigen Ansätze zur Lösung der Problematik unrechtmäßiger Anerkennungen. ${ }^{528}$

Diese Schwächen werden von der deklaratorischen Theorie aufgefangen. Die Existenz eines Staates sei ein Fakt, welcher mit oder ohne Anerkennung durch andere Staaten gegeben sei. ${ }^{529}$ Eine Anerkennung bestätigt nur das Vorliegen der

\footnotetext{
${ }^{524}$ Crawford, The creation of states in international law, S. 19-28.

525 H. Lauterpacht, Recognition in international law, 1948, S. 55.

${ }^{526}$ P. K. Menon, The law of recognition in international law, 1994, S. 8.

${ }^{527}$ Crawford, The creation of states in international law, S. 20.

528 Ebd., S. 20-22.

${ }^{529}$ Menon, The law of recognition in international law, S. 18.
} 
notwendigen Voraussetzungen eines Staatsgebiets, Staatsvolks und der Staatsgewalt, die einen Staat ausmachen. Der Bewertungsmaßstab ist dabei die Effektivität der Staatlichkeit, die apolitisch und unabhängig von Legitimitätsstandards allein nach der formellen Faktenlage festgestellt werden kann. ${ }^{530}$ Im Sinne der naturrechtlichen Perspektive garantiert eine bloß deklaratorische Wirkung der Staatenanerkennung die Prinzipien der souveränen Gleichheit der Staaten und ihrer friedlichen Koexistenz. 531

Allerdings, dies zeigen die jüngeren Fälle der Staatengenese, ist eine objektive Bewertung der Effizienz der Staatlichkeit kaum möglich, so dass die Staatenanerkennung in der Praxis als politisches Instrument zur Steuerung von Konflikten eingesetzt wird. ${ }^{532}$ Es sei utopisch, dass sich jegliche staatsähnlichen Gebilde durch eine Selbstdeklaration als ,Staat ${ }^{`}$ den Status als Völkerrechtssubjekt selbst zusprechen könnten. Die Rechtspersönlichkeit eines Staates sei rechtlich bedingt und kann sich nicht allein aus der Natur ableiten, so dass völkerrechtliche Bewertungsmaßstäbe angelegt werden können,, ${ }^{53}$ um die Legalität bzw. Illegalität einer Staatsgründung bewerten und damit über die Anerkennung entscheiden zu können. 534

Nachdem im 19. Jahrhundert, einer Zeit der Dominanz der europäischen Staaten als ,closed club' der Völkerrechtssubjekte, die konstitutive Theorie vorherrschte, gewann im Laufe des 20. Jahrhunderts die deklaratorische Theorie an Bedeutung. ${ }^{535}$ Sie lieferte einen schlüssigeren Rahmen für die Beurteilung der Genese neuer Staaten in Verbindung mit dem Konzept der Selbstbestimmung und dem Prozess der Dekolonisation. Die Staatenpraxis der Anerkennung nach dem Zweiten Weltkrieg, und zwar insbesondere in Bezug auf die Sezessionen nach dem Fall der Mauer in den Staaten des ehemaligen Ostblocks, wird heute wegen ihrer politischen Steuerung im Krisenmanagement als eine Kollektivierung und Konditionalisierung der Anerkennung bewertet. ${ }^{536}$ Die Anerkennung neuer Staaten wird durch politische Absprachen und im Rahmen inter- und supranationaler Organisationen kollektiviert. Dabei werden den im Entstehen begriffenen Staaten individuell konkrete Konditionen für eine Anerkennung auferlegt und somit Einfluss auf Prozesse einer Sezession genommen, um nationale und internationale Konflikte beeinflussen zu können.

\footnotetext{
530 Urs Saxer, Die internationale Steuerung der Selbstbestimmung und der Staatsentstebung, 2010, S. $704 \mathrm{f}$.

531 Menon, The law of recognition in international law, S. $22 \mathrm{ff}$.

532 Saxer, Die internationale Steuerung der Selbstbestimmung und der Staatsentstehung, S. 707.

533 So einigten sich die EG-Mitgliedstaaten 1991 beispielsweise auf einen Kriterienkatalog zur Anerkennung neuer Staaten. Siehe dazu: Christian Hillgruber, Die Aufnabme neuer Staaten in die Völkerrechtsgemeinschaft, 1998, S. 744.

534 H. Lauterpacht, „Recognition of States in International Law“, Yale Law Journal, Nr. 53 (1944), S. 428; Saxer, Die internationale Steuerung der Selbstbestimmung und der Staatsentstehung, S. 708.

535 Menon, The law of recognition in international law, S. 26.

536 Saxer, Die internationale Steuerung der Selbstbestimmung und der Staatsentstebung, S. 718-735.
} 
Im Folgenden sollen die Argumente dieser Debatte für die Fragen der Anerkennung von substaatlichen Gruppen fruchtbar gemacht werden. Zwar geht es bei der Anerkennung substaatlicher Gruppen nicht wie bei Staaten um die grundlegende Frage der Völkerrechtssubjektivität. Der Status als ,Minderheiten', ,indigenes Volk' oder , lokale Gemeinschaft' bedingt jedoch ebenfalls den rechtlichen Status einer Gruppe im Völkerrecht.

\section{Anerkennung substaatlicher Gruppen im Über-/Unterordnungsverhältnis}

Die Übertragung der Argumente der Debatte über die rechtliche Bedeutung der Anerkennung bei der Genese von Staaten auf die Situation substaatlicher Gruppen kann nur unter Beachtung der strukturellen Unterschiede der Beziehungen der beteiligten Subjekte zueinander erfolgreich sein. Während sich die Staatenanerkennung auf nur einer Ebene abspielt, also bestehende Völkerrechtssubjekte neue Staaten in ihrer Reihe als statusgleiche Völkerrechtssubjekte aufnehmen, vollzieht sich die Anerkennung substaatlicher Gruppen vor dem Hintergrund eines Über-/Unterordnungsverhältnisses der Gruppen zu ihrem Heimatstaat.

Das Über-/Unterordnungsverhältnis substaatlicher Gruppen zu ihrem Heimatstaat ergibt sich zunächst aus ihrem rechtlichen Status im Rahmen der nationalen Rechtsordnung und bedingt damit ihren völkerrechtlichen Status gegenüber internationalrechtlichen Akteuren. Die bestehenden Völkerrechtssubjekte entscheiden konstitutiv über das $\mathrm{Ob}$ und den Umfang der Rechte, die sie gewähren oder übertragen, und welchen Status sie neuen Subjekten dadurch einräumen möchten. Das Über-/Unterordnungsverhältnis der Gruppen zu ihrem Heimatstaat ergibt sich dabei nicht aus den Definitionsansätzen der Konzepte substaatlicher Gruppen, sondern aus den Konstruktionen der jeweiligen rechtlichen Regelungsgehalte, die für Minderheiten, indigene Völker und lokale Gemeinschaften getroffen wurden und werden.

Minderheiten stehen mit ihren Rechten auf die Nutzung ihrer Sprache, den Schutz ihrer Kultur und der besonderen Religionsfreiheit ihrem Heimatstaat als Anspruchssteller gegenüber. Dieser muss die jeweilige Sprache als Amtssprache festlegen, Schulen und Kulturinstitutionen gegebenenfalls erlauben und gegebenenfalls finanziell fördern sowie Eingriffe in die Religionsfreiheit auf solche Fälle beschränken, in den dies in Abwägung zu anderen Menschenrechten gerechtfertigt ist. ${ }^{537}$ Kulturell distinkte Gruppen, wenn sie nicht als Minderheiten anerkannt sind, können solche Rechte nicht gegenüber staatlichen Institutionen geltend machen. Es obliegt ausschließlich dem Heimatstaat, die notwendigen Schritte zur Ausgestaltung der Minderheitenrechte zu ergreifen. Eine Umsetzung der rechtlichen Gehalte des Minderheitenstatus' ist ohne seine Mitwirkung nicht denkbar. Im Vergleich zur Staatenanerkennung wird deutlich, dass auf die Anerkennung

537 Siehe dazu „A. III. Inhalt des internationalen Minderheitenschutzes“ auf S. 21 
nicht verzichtet werden kann. Während neue Staaten im Sinne der deklaratorischen Theorie unabhängig von der Anerkennung durch Drittstaaten ihre Staatlichkeit umsetzen können, ist dies für die Ausübung des Minderheitenschutzes nicht möglich. Kulturell distinkte Gruppen sind auf die Anerkennung als Minderheiten durch ihren Heimatstaat angewiesen, wenn sie im Rahmen seiner Rechtsordnung ihre Rechte umgesetzt sehen möchten.

Gleiches gilt für die Rechte indigener Völker. Die Forderung einer indigenen Gruppe nach Selbstbestimmung kann zwar in Ausnahmefällen die Sezession von ihrem Heimatstaat bedeuten, 538 diese würde aber nach den Regeln der Staatenanerkennung beurteilt werden. Handelt es sich jedoch um eine Forderung nach Selbstbestimmung im Rahmen der menschenrechtlichen Garantien, wie sie insbesondere in der UNDRIPs niedergelegt wurde, ${ }^{539}$ bedarf es der Übertragung von Regelungs- und Gesetzgebungskompetenzen vom und durch den Heimatstaat auf die Gruppe. Die Gruppe leitet ihre Selbstbestimmung, ihre Regelungskompetenzen und somit ihren rechtlichen Status von ihrem Heimatstaat ab. Andernfalls würde ein Ausbrechen aus der staatlichen Rechtsordnung am Gewaltmonopol des Heimatstaates scheitern. Kulturell distinkte Gruppen bedürfen demnach seiner Anerkennung als ,indigenes Volk', um selbst über bestimmte Inhalte bestimmen zu können. Für die Umsetzung von Selbstbestimmungsrechten bedarf es notwendigerweise einer Anerkennung als, indigenes Volk 6 seitens des Heimatstaates, welcher somit konstitutive Bedeutung zukommt.

Ebenso sind lokale Gemeinschaften für die Umsetzung der ihnen völkerrechtlich zugeschriebenen Rechte von ihren Heimatstaaten abhängig. Sie erhalten ihre Rolle im Beteiligungsverfahren des ihnen garantierten prior informed consent nur insoweit diese durch ihre Heimatstaaten umgesetzt werden. ${ }^{540}$ Das Nagoya Protokoll weist insoweit die entscheidende Rolle den Heimatstaaten zu. Die zentrale Rolle der Staaten zeigt sich auch in $\operatorname{der} C B D$, welche genetische Ressourcen normativ den Staaten und nicht den lokalen Gemeinschaften zuordnet. Die Rolle lokaler Gemeinschaften ist somit von der nationalgesetzlichen Ausgestaltung durch den Heimatstaat abhängig.

Die Struktur der Rechte substaatlicher Gruppen gibt das Verhältnis von Anspruchssteller und -gegner in einem Über-/Unterordnungsverhältnis der Gruppen zu ihrem Heimatstaat vor. Die bestehenden Völkerrechtssubjekte entscheiden konstitutiv über das $\mathrm{Ob}$ und den Umfang der Rechte, die sie gewähren oder übertragen und welchen Status sie neuen Subjekten dadurch einräumen möchten.

\footnotetext{
538 Siehe dazu „B. III. 1. Politische Rechte“ auf S. 52.

539 Siehe dazu „B. III. Das Selbstbestimmungsrecht indigener Völker“ auf S. 50.

540 Siehe dazu „C. IV. Nationalgesetzlicher Rahmen und Rechte geistigen Eigentums“ auf S. 78.
} 


\section{Selbstidentifikation und Anerkennung}

Die für substaatliche Gruppen anerkannte Selbstidentifikation kultureller Distinktion stellt wie auch in der Debatte um die Staatenanerkennung die Frage der Praktikabilität einer solchen einseitigen Erklärung. Für potentielle Staatengebilde sei es utopisch, dass sie sich durch eine Selbstdeklaration als ,Staat ${ }^{6}$ den Status als Völkerrechtssubjekt selbst zusprechen könnten. Gleiches gilt für substaatliche Gruppen. Wäre allein die kollektive Selbstidentifikation einer kulturellen Distinktion zur Konstituierung einer substaatlichen Gruppe im Sinne eines der drei Konzepte ausreichend, könnte dies die Genese einer Vielzahl neuer Gruppen bedeuten. Ohne konstitutiv notwendige Anerkennung durch irgendjemanden könnte sich eine nicht zu kontrollierende Vielzahl neuer Subjekte bilden, welche entsprechende Rechte für sich geltend machen würden. ${ }^{541}$ Zudem könnten substaatliche Gemeinschaften ohne notwendiger konstitutiver Anerkennung über die Selbstidentifikation als ,Minderheiten', ,indigene Völker' und ,lokale Gemeinschaften' kumulativ die Rechtspositionen aller Konzepte beanspruchen, sofern sie die positivrechtlich festgelegten Merkmale aufweisen. Aus der Vielfalt kulturell distinkter Gruppen ergäbe sich eine Kakophonie sich mehrfach überlagernder Ansprüche einer Vielzahl von Subjekten.

Zwar besteht Konsens hinsichtlich der Selbstidentifikation kultureller Distinktion substaatlicher Gruppen, die sich in allen Definitionsansätzen für jedes der drei Konzepte findet. ${ }^{42}$ Diese allein kann in der Praxis allerdings nicht zu tragfähigen Lösungen führen. Eine einseitige Entscheidung substaatlicher Gruppen über ihren Rechtsstatus als ,Minderheit', ,indigenes Volk ${ }^{6}$ oder , lokale Gemeinschaft ${ }^{\star}$ durch bloße Selbstidentifikation der Gruppe, ohne dass das jeweils rechtlich verpflichtete Gegenüber oder ein Dritter beteiligt wäre, ist ebenso utopisch, wie dies für potentielle Staatengebilde in den Debatten um die Staatenanerkennung festgestellt wurde.

In Anknüpfung an die Ergebnisse zur Praktikabilität der Anerkennung in der Staatenanerkennungsdebatte lassen sich die Argumente für einer individuell konditionalisierte, konstitutiv notwendige Anerkennung übertragen. Die individuelle konstitutive Anerkennung des Rechtsstatus' substaatlicher Gruppen ergänzt die anerkannte Schlüsselrolle der Selbstidentifikation zur Bestimmung substaatlicher Gruppen. Ebenso wie bei der Frage der Staatenanerkennung lassen sich im Zuge eines Anerkennungsprozesses notwendige Kriterien individuell vereinbaren, die im konkreten Kontext notwendig sind. So ließen sich auch Voraussetzungen in den Anerkennungsprozess einstellen, die von übergeordnetem Interesse sind und über das bloße erfüllen von Definitionsmerkmalen hinausgehen. Aspekte der

541 Timo Makkonen, Identity, Difference and Otherness: The Concepts of „People“, Indigenous People' and „Minority " in International Law, 2000, S. 53.

${ }^{542}$ Siehe dazu „D. Selbstidentifikation kultureller Distinktion als Kern der Definitionsansätze“ auf S. 114. 
Konfliktbewältigung im lokalen und regionalen Kontext, notwendige Stabilität und Verfasstheit der Gruppe nach innen und außen, ausreichende Formen einer gegebenenfalls demokratischen Repräsentation, Menschenrechte und weitere rechtsstaatliche Garantien könnten wie bei der Staatenanerkennung für eine Anerkennung substaatlicher Gruppen eingestellt werden.

Gleichzeitig löst die Selbstidentifikation von Gruppen als ,Minderheit‘, ,indigenes Volk' oder ,lokale Gemeinschaften' und eine entsprechende Anerkennung die Problematik der rechtlich möglichen, überlagernden Anwendbarkeit der unterschiedlichen Konzepte. Die Selbstidentifikation ist der Ansatzpunkt zur Lösung der Problematik der mangelnden Definitionen und Unterscheidbarkeit der unterschiedlichen Konzepte in der Praxis. Zunächst zeigt die Selbstidentifikation, ob eine Gruppe im Kontext ihres Heimatstaates ihre kulturelle Distinktion behaupten und entsprechende Rechte geltend machen möchte. Dabei ergibt sich auch eine Entscheidung für die Anwendung eines der verschiedenen Konzepte, da die Gruppe sich als ,Minderheit', indigenes Volk' oder, lokale Gemeinschaften' positionieren muss.

Als Gegenstück zur Selbstidentifikation wirkt dann die Anerkennung. Im Zuge der Interaktionen substaatlicher Gruppen mit ihren Heimatstaaten, internationalen Organisationen, Drittstaaten und weiteren Akteure zeigt sich, ob sie im Sinne des vorgebrachten Konzepts anerkannt werden. Mangels einer allgemein anerkannten, abstrakt-generellen Definition mit der eine Bestimmung substaatlicher Gruppen als ,Minderheit', ,indigenes Volk' oder ,lokale Gemeinschaften' möglich ist, vollzieht sich in diesem Prozesses der Aushandlung, also im Dialog der Selbstidentifikation der Gruppe und einer Anerkennung durch das jeweilige Gegenüber, die konkrete rechtliche Einordnung zur Bestimmung der Statusfrage.

\section{Existenz substaatlicher Gruppen und Anerkennung}

Bei der rechtlichen Bewertung der Anerkennung substaatlicher Gruppen ist zwischen dem Bestehen kollektiver Identität, also der Existenz einer Gruppe auf der einen Seite und dem Rechtsstatus dieser Gruppe im Sinne der drei völkerrechtlichen Kategorien auf der anderen Seite, zu unterscheiden. Für die Bildung einer kollektiven Identität ist die Anerkennung durch die Regierung des Heimatstaates keine zwingend notwendige Voraussetzung. Kollektive Identitäten substaatlicher Gruppen bilden sich unabhängig von staatlichen Einflüssen und bestehen auch ohne weitere Anerkennung von staatlicher Seite fort. So schreibt auch der Sonderberichterstatter Asbjørn Eide: „Whether a given minority exists, does not depend on recognition by the State. Its existence depends on objective and subjective factors. ${ }^{"}{ }^{4} 3$

\footnotetext{
543 Sub-Commission on the Prevention of Discrimination and Protection of Minorities, Asbjørn Eide, „Preliminary Report submitted by the Special Rapporteur Mr. Asbjørn Eide, on possible
} 
Asbjørn Eide argumentiert dazu weiterführend, dass der Minderheitenschutz nicht von einer Anerkennung nach nationalem Recht abhängen kann, sondern sich automatisch aus der Existenz der Gemeinschaft ergibt. ${ }^{544}$ Diese Schlussfolgerung wird in Hinblick auf Ziel und Zweck der Regelungen zum Schutz von Minderheiten und in Anbetracht der Systematik der Menschenrechte unterstützt. ${ }^{545}$ Die Beispiele verweigerter Anerkennungen zeigen jedoch, dass die gegenwärtige völkerrechtliche Praxis nicht von einem Automatismus der Anerkennung ausgeht. ${ }^{546}$ Ebenso wenig findet sich eine positivrechtlich verankerte Pflicht zur Anerkennung substaatlicher Gruppen, sollten sich Gemeinschaften als ,Minderheit', ,indigenes Volk ${ }^{\varsigma}$ und ,lokale Gemeinschaft ${ }^{`}$ identifizieren. Die faktische Existenz einer substaatlichen Gruppe hat im geltenden Recht nicht automatisch einen rechtlichen Status in den Kategorien, die das Völkerrecht normiert, zur Folge, auch wenn dies nach Ziel und Zweck der Regelungen für substaatliche Gruppen wünschenswert wäre.

Die Notwendigkeit der Anerkennung wird deutlich im Vergleich zu weniger konkret konstituierten Gruppen kultureller Distinktion. Wenn sich durch Migration, veränderte Grenzen oder kulturelle Veränderungen in Teilen einer Gesellschaft neue kulturell distinkte Gruppen in einem Staat bilden, ist nicht unmittelbar deutlich, ob und wie diese Gruppen ihre kulturelle Distinktion umsetzen möchten. Kulturen verändern sich konstant und über Zeiträume, die eine zeitliche Bestimmung, wann eine Gruppe für einen völkerrechtlichen substaatlichen Gruppenstatus kulturell unterschiedlich genug ist, unmöglich machen. Für die Anwendbarkeit entsprechender Rechte im Rahmen der nationalen Rechtsordnung bedarf es daher zunächst der Selbstidentifikation der Gruppe als ,Minderheit, ,indigenes Volk ${ }^{6}$ oder ,lokale Gemeinschaft' und der Anerkennung durch den Heimatstaat. Der zitierten Aussage des Sonderberichterstatters Asbjørn Eide ist somit in Hinblick auf den Sinn und Zweck der Regelungen für substaatliche Gruppen zuzustimmen. $\mathrm{Ob}$ eine Minderheit, wie er es sagt, existiert, sollte keine Frage der Anerkennung sein. Ob allerdings eine Gruppe, die einen gewissen Grad an kultureller Distinktion besitzt, eine ,Minderheit', ein ,indigenes Volk' oder eine ,lokale Gemeinschaft im völkerrechtlichen Sinne ist, bedarf jedoch einer Entscheidung. Um gerade diesen Schritt rechtlich greifbar zu machen, bedarf es konkreter Regelungen der Anerkennung.

ways and means of facilitating the peaceful and constructive solution of problems involving minorities“, UN Doc. E/CN.4/Sub.2/1990/46, Abs. 16.

544 Sub-Commission on the Prevention of Discrimination and Protection of Minorities, Asbjørn Eide, „Possible Ways and Means of Facilitating the Peaceful and Constructive Solution of Problems Involving Minorities“, UN Doc. E/CN.4/Sub.2/1993/34 and Add. 1-4, Abs. 113.

545 Sarah Pritchard, Der völkerrechtliche Minderheitenschutz, 2001, S. 206; Sigrid Boysen, Europäische Charta der Regional- oder Minderbeitensprachen: Handkommentar, 2011, Art. 7, Rn. 6; Makkonen, Identity, Difference and Otherness, S. 53.

546 Thornberry, International law and the rights of minorities, S. $157 \mathrm{f}$. 


\section{Die Relativität der Anerkennung}

Auf internationaler Ebene hat die Unterscheidung zwischen der Existenz substaatlicher kollektiver Identitäten auf der einen Seite und der Anerkennung oder Nicht-Anerkennung von Gruppen im Sinne eines der drei Konzepte auf der anderen komplexe Folgen der Relativität der Anerkennung. Erkennt der Heimatstaat eine substaatliche Gruppe nicht im Sinne eines der Konzepte an, erfolgt aber eine Anerkennung durch Drittstaaten oder internationale Organisationen, konstituieren sich jeweils rechtliche Beziehungen zwischen diesen und der Gruppe, was ein Gewirr unterschiedlicher Anerkennungsbeziehungen zur Folge hat. Im Kontext der Debatte der Staatenanerkennung beschrieb Hans Kelsen die Relativität der Anerkennung folgendermaßen:

By the legal act of recognition the recognized community is brought into legal existence in relation to the recognizing state, and thereby international law becomes applicable to the relations between these states. Hence the recognition has a specifically constitutive character. ${ }^{547}$

Der konstitutive Charakter der Anerkennung hat also ein komplexes Geflecht von Anerkennungsbeziehungen zufolge. Darauf aufbauend wird argumentiert, dass die Vielzahl unterschiedlicher Meinungen innerhalb der Staatengemeinschaft mangels einer zentralen Entscheidungsinstanz zu Rechtsunsicherheit führe. Der Anerkennung solle somit nur deklaratorische Wirkung zukommen, um dies zu verhindern.

Bei einer Übertragung der Argumente der Auseinandersetzung zur Relativität der Anerkennung auf die Situation substaatlicher Gruppen ist zu beachten, dass bei substaatlichen Gruppen die rechtlichen Verhältnisse der Zuordnung einer letzten Entscheidungsinstanz geklärt sind. Letztlich ermöglicht nämlich ihre geographische Lage eine aus völkerrechtlicher Perspektive rechtlich eindeutige Zuordnung in Bezug zu ihrem Heimatstaat. Die Vielzahl der beteiligten Akteure im Geflecht der Anerkennungsstrukturen schafft zwar einen komplexen Diskurs. Dieser kann jedoch weder das rechtliche Über-/Unterordnungsverhältnisses der Gruppe in ihrem Heimatstaat noch ihre geographische Lage in demselben verschleiern. Eine Theorie der konstitutiven Anerkennung substaatlicher Gruppen durch den Heimatstaat entkräftet somit das Argument der Rechtsunsicherheit.

Die Praxis der Anerkennung auf internationaler Ebene zeigt jedoch, dass sich solch ein Gewirr unterschiedlicher Anerkennungsbeziehungen entwickelt. Der Anerkennung durch internationalrechtliche Akteure kommt in der bilateralen Beziehung zwischen Drittstaat oder internationaler Organisation und der Gruppe

\footnotetext{
547 Hans Kelsen, „Recognition in International Law, Theoretical Observations“, American Journal of International Law, Vol. 35, Nr. 4 (1941), S. 609.
} 
ebenfalls konstitutive Wirkung zu, unabhängig davon, ob der Heimatstaat ebenfalls eine Anerkennung ausgesprochen hat. Diese Relativität der Anerkennung, wie sie Kelsen bereits für die Staatenanerkennung gezeigt hat, zwingt allerdings im Fall der substaatlichen Gruppen den Heimatstaat über die internationale Politik sich mit jeweils internationalrechtlich anerkannten Gruppen auch auf nationaler Ebene auseinanderzusetzen. Die geographische Zuordnung einer substaatlichen Gruppe zu einem Heimatstaat nimmt diesen in die Pflicht über eine Anerkennung zu entscheiden. Der Heimatstaat kann für die substaatlichen Gruppen auf seinem Territorium durch seine Anerkennung ihren internationalrechtlichen Status bestimmen. Anders als bei der Staatenanerkennung kann das Gewirr unterschiedlicher Anerkennungsbeziehungen dadurch aufgelöst werden.

\section{Funktioneller Inhalt der Anerkennung durch internationale Akteure}

Die Relativität der Anerkennung, die sich aus der konstitutiven Wirkung der Anerkennung zwischen der Gruppe und internationalen Akteuren ergibt, erfordert eine differenzierende Perspektive auf den Umfang und den Inhalt der Anerkennung. Während die Anerkennung durch den Heimatstaat eine umfassende Rechtsposition postuliert, beschränkt sich die Anerkennung einer substaatlichen Gruppe durch andere internationalrechtliche Akteure auf die Anerkennung der Existenz sowie den funktionellen Kontext, in dem er der Gruppe gegenübersteht. Dies bedeutet beispielsweise, dass das Human Rights Committee Minderheiten oder indigene Völker als Beschwerdeführer eines Verfahrens anerkennen und eine Verletzung ihrer Rechte durch ihren Heimatstaat feststellen kann. Die Umsetzung dieser Entscheidung und Gewährung der Rechte des Artikels 27 IPbpR kann jedoch nur durch den Heimatstaat erfolgen. Die Anerkennung des Human Rights Committees ist auf den funktionellen Kontext des Beschwerdeverfahrens beschränkt und wirkt nur politisch auf eine Anerkennung der Gruppe sowie etwaiger Rechtspositionen auf nationaler Ebene zurück. Einen rechtlichen Automatismus der Anerkennung kann von einer internationalen Beschwerdeinstitution oder Drittstaaten nicht ausgelöst werden.

\section{Politik der Anerkennung}

Die Anerkennung substaatlicher Gruppen, wie sie mangels abstrakt-genereller Definitionen gegenwärtige Praxis und für den rechtlichen Status von konstitutiver Bedeutung ist, unterwirft Minderheiten, indigene Völker oder lokale Gemeinschaften den politischen Erwägungen und Machtkämpfen auf nationaler und internationaler Ebene. Zwar sind die Entwicklungen des modernen Minderheitenrechts von dem Umstand geprägt, dass die Geltung der Minderheitenschutzverpflichtungen gerade nicht von der staatlichen Anerkennung abhängig 
gemacht werden soll, da somit Schutzverpflichtungen relativiert und der Verpflichtungscharakter „bis zur Unkenntlichkeit reduziert werden“ würden. ${ }^{548}$ Das Völkerrecht trifft jedoch keine Regelungen in welcher Art und Weise die Anerkennungsprozesse ausgestaltet sein sollen. Vielmehr zeigt sich eine Bandbreite möglicher Prozesse, die von impliziten politischen Anerkennungen bis hin zu expliziten rechtlichen Regelungen reicht. Der Prozess der Anerkennung kann sich über mehrere Jahrzehnte erstrecken oder einem festen, institutionalisierten Rahmen unterliegen, der konkrete Vorgaben zu Voraussetzungen und zulässigen Beweismitteln macht. Neben den Heimatstaaten der Gruppen spielen dabei auch Drittstaaten, internationale Organisationen, Nichtregierungsorganisationen oder auch private Akteure eine Rolle.

Die Politik der Anerkennung eröffnet einerseits die Möglichkeiten individueller Lösungen, die ähnlich den Prozessen der Staatenanerkennung auch bewusst zur Steuerung von Konflikten eingesetzt werden kann. ${ }^{549}$ Andererseits offenbart sie das Machtgefälle zwischen den nach Anerkennung strebenden Gruppen und ihren Heimatstaaten, da diese letztlich entscheiden können, entsprechende Rechte zu gewähren oder nicht. Dabei sind die Staaten wiederum Teil des weiteren internationalen Diskurses, in dem sie sich gegebenenfalls dem politischen Druck anderer Akteure beugen müssen. Gleichzeitig sind die Staaten aber die entscheidenden Akteure der Anerkennung auf ihrem Territorium, wobei der fehlende internationalrechtliche Rahmen im Anerkennungsprozess letztlich die Rolle des Heimatstaates kräftigt und so das Machtgefüge, gegen welches die Gruppenrechte eigentlich einen Ausgleich schaffen sollen, reproduziert und verschärft. Es existieren keine rechtlichen Vorgaben oder Voraussetzungen, die eine Beteiligung der Gruppen oder auch Dritter am Diskurs um den rechtlichen Status der Gruppen garantieren und die Entscheidungsgewalt der Staaten konkretisieren oder gar beschränken.

\section{Problematik verweigerter Anerkennung}

Wird die konstitutive Wirkung der Anerkennung für den rechtlichen Status substaatlicher Gruppen getrennt von der Existenz substaatlicher kollektiver Identitäten betrachtet, ergeben sich, wie auch im Rahmen der konstitutiven Theorie der Staatenanerkennung, die Probleme unrechtmäßiger und verweigerter Anerkennung. „,If] any entity bears the marks of statehood, other states put themselves at risk legally if they ignore the basic obligations of state relations. "550 Durch die Relativität der Anerkennungsbeziehungen auf internationaler Ebene können den Heimatstaaten im Rahmen der internationalen Berichts- und Beschwerdeverfahren etwaige Verletzungen der Rechte von Minderheiten, indige-

548 Peter Hilpold, Modernes Minderbeitenrecht: eine rechtsvergleichende Untersuchung des Minderheitenrechtes in

Österreich und in Italien unter besonderer Berücksicbtigung völkerrecbtlicher Aspekte, 2001, Art. 7, Rn. 6.

549 Saxer, Die internationale Steuerung der Selbstbestimmung und der Staatsentstebung, S. $731 \mathrm{ff}$.

${ }^{550}$ Ian Brownlie, Principles of public international law, 2008, S. 90. 
nen Völkern oder lokalen Gemeinschaften vorgeworfen werden. Die Konflikte der Nicht-Anerkennung können sich so auf die Beziehung zwischen den Heimatstaaten und Dritten völkerrechtlichen Akteuren verlagern.

VIII. Zwischenergebnis

Ausgangspunkt einer völkerrechtlichen Bewertung der Anerkennung substaatlicher Gruppen ist die Praxis der Anwendung der Konzepte substaatlicher Gruppen. Die obige Darstellung der Anwendung der Konzepte unter einer Perspektive der Anerkennung öffnet den Blick auf die unterschiedlichen Verfahren und Formen der Anerkennung, wie sie heute mangels völkerrechtlicher Vorgaben weltweit umgesetzt wird. Die Anerkennung wirkt als Pendant zur Selbstidentifikation kultureller Distinktion, die in den Diskussionen zur Bestimmung substaatlicher Gruppen anhand abstrakt-genereller Definitionen als allgemeiner Kern zugrunde gelegt wird. Es obliegt den Gruppen, sich entsprechend ihrer politischen Forderungen im Sinne der unterschiedlichen Konzepte gegenüber ihrem Heimatstaat zu positionieren. ${ }^{551}$ Sind es Ansprüche auf Vorteilsteilhabe und Zustimmungsrechte für den Zugang zu traditionellem Wissen in Bezug auf pflanzengenetische Ressourcen, sind die umweltvölkerrechtlichen Instrumente für ,lokale Gemeinschaften` einschlägig. Rechte zum Schutz der Sprache, Religion und kulturellen Integrität werden ,Minderheiten' gewährt. Der Anspruch auf Selbstbestimmung konkretisiert sich in der UNDRIPs für, indigene Völker'. Die Anerkennung führt dann zur Anwendbarkeit dieser Konzepte und bestimmt den Rechtsstatus der Gruppen als ,Minderheit', ,indigenes Volk` oder ,lokale Gemeinschaft'.

Dabei ist zwischen der Existenz kollektiver Identität und dem Rechtsstatus der Gruppen zu unterscheiden. Kulturell distinkte Gruppen existieren ohne Anerkennung seitens ihrer Heimatstaaten. Ihr Rechtsstatus im Sinne der völkerrechtlichen Konzepte im Staat und auch international hängt jedoch von der konstitutiven Anerkennung seitens des Heimatstaates oder zumindest des jeweiligen Gegenübers in einer rechtlichen Beziehung ab. Ein Gruppenrechtsstatus ist unter der gegenwärtigen Rechtslage kein Automatismus, sondern ergibt sich erst durch die Anerkennung. Für die Frage der Existenz kollektiver Identitäten kommt der Anerkennung somit bloß deklaratorische Bedeutung zu. Für den rechtlichen Status dieser Gruppen hingegen ist die Anerkennung konstitutiv.

Für die innerstaatlichen Beziehungen ergibt sich die konstitutive Wirkung der Anerkennung aus dem Über-/Unterordnungsverhältnis substaatlicher Gruppen gegenüber ihren Heimatstaaten. Jeglicher rechtliche Status substaatlicher Gruppen in der nationalen Rechtsordnung ist auf die staatliche Souveränität zur Regelung innerer Angelegenheiten zurückzuführen. Mangels expliziter Primärquellen auf

551 Barsh, „Indigenous Peoples“, S. 836. 
internationaler Ebene gibt es auch keine völkerrechtlichen Regelungen, die bei dieser Frage in nationales Recht hineinwirken könnten.

Auf internationaler Ebene hingegen, wo substaatliche Gruppen gegenüber anderen internationalrechtlichen Akteuren auftreten, kann eine konstitutive Anerkennung für bestimmte Rechtspositionen auch durch Drittstaaten oder internationale Organisationen erfolgen. Trotz der eindeutigen Zuordnung substaatlicher Gruppen zu ihrem Heimatstaat kann sich dadurch ein Gewirr von unterschiedlichen Anerkennungsbeziehungen bilden. Offen ist, wie diese unterschiedlichen Anerkennungsbeziehungen politisch auf den Rechtstatus nach nationalem Recht Einfluss nehmen.

Die zunehmende Genese neuer substaatlicher Gruppen, die daraus resultierenden komplexen Akteurskonstellationen und die bisher völkerrechtlich nicht geregelten Anerkennungsverfahren werfen neue Fragen für weiterführende Untersuchungen auf. Auch wenn eine weiterführende Untersuchung der Frage der Staatenanerkennung mittlerweile als fruchtlos oder gar ablenkend bewertet wird, ${ }^{552}$ die Erfahrungen mit Prozessen der Staatenanerkennung können für die Beantwortung mancher dieser aufkommenden Fragen als Vorbild dienen. Beispielsweise formulierte die Europäische Gemeinschaft in ihrer Declaration on the ,Guidelines on the Recognition of New States in Eastern Europe and in the Soviet Union "normative Maßstäbe zur Staatenanerkennung in Reaktion auf die Entwicklungen nach dem Fall der Mauer. ${ }^{553}$ Aspekte der demokratischen Legitimation, die Verpflichtung zu menschenrechtlichen Garantien und zur Teilnahme an Verfahren der friedlichen Streitbeilegung gaben der Anerkennungspraxis der EG einen zunehmend rechtlichen Gehalt. ${ }^{554}$ Für eine Beurteilung von best practices der gegenwärtigen Praxis der Anerkennung substaatlicher Gruppen und der konditionalisierten Anerkennung von Staaten bedarf es weiterer Studien.

Die Ausführungen hier haben bereits gezeigt, dass die Anerkennung substaatlicher Gruppen, also die gegenwärtige Form der Anwendung der Konzepte, in verschiedensten Formen umgesetzt wird. Die Gestaltung der Prozesse und meist auch die finalen Entscheidungen über den Rechtsstatus obliegen dabei der nationalen Politik. Die Perspektive der Anerkennung macht somit deutlich, dass es hinter all den bisher erfolglosen Debatten um mögliche Definitionen heute letztlich die Staaten sind, die über den Rechtsstatus kulturell distinkter Gruppen entscheiden. Angesichts der Tatsache, dass der Minderheitenschutz, das Selbstbestimmungsrecht indigener Völker und die Rechte auf Teilhabe und Mitbestimmung bei Fragen zu genetischen Ressourcen lokaler Gemeinschaften gerade die

\footnotetext{
552 Ian Brownlie, „Recognition in theory and practice“, The British yearbook of international law, 1983, S. 197.

${ }^{553}$ Europäische Gemeinschaft, „Declaration on the 'Guidelines on the Recognition of New States in Eastern Europe and in the Soviet Union"“, 31 ILM 1485, 16. Dezember 1991.

554 Saxer, Die internationale Steuerung der Selbstbestimmung und der Staatsentstebung, S. 718.
} 
Rechtsposition dieser Gruppen gegenüber ihren Heimatstaaten stärken soll, offenbaren sich die gegenläufigen Interessen: Um bestimmte Rechte einer kulturell distinkten Gruppe nicht gewähren zu müssen, erkennen Staaten diese nicht mit dem entsprechenden Rechtsstatus an. Vorgebrachten Widersprüchen gegen verweigerte Anerkennung wurde bisher mit Verweis auf entsprechend politisch geformte Definitionen begegnet, die wegen mangelnder Eigenschaften einer Gruppe die Anwendung notwendiger Rechte verbiete. Die Idee von Rechten für substaatliche Gruppen gegenüber ihren Heimatstaaten wird - und dies macht die Perspektive der Anerkennung deutlich - in der gegenwärtigen Praxis, wo die Anwendung dieser Rechte von der konstitutiven Anerkennung durch die Staaten abhängt, nur sehr eingeschränkt gewährleistet.

Die Diskussionen um abstrakt-generelle Definitionen können Erwägungen zu rechtlichen Vorgaben für die Prozesse der Anwendung nur schwerlich einbeziehen. Es bedarf daher des hier aufgezeigten Perspektivenwechsels auf die Prozesse und die Politik der Anerkennung, um die Diskussionen für einen effektiveren Minderheitenschutz, eine besser Umsetzung der Rechte indigener Völker und eine umfassendere Einbeziehung lokaler Gemeinschaften im Umweltrecht voranzubringen.

Die Politik der Anerkennung, die mit den Problemen verweigerter Anerkennungen einhergeht, bildet das Pendant zur Selbstidentifikation und reguliert dadurch die Genese neuer rechtlich anerkannter, substaatlicher Gruppen. Eine zunehmende Verrechtlichung anhand normativer Maßstäbe und einer Konditionalisierung, wie sie sich bei der Staatenanerkennung abzeichnet, ${ }^{555}$ könnte dem Zusammenspiel von Selbstidentifikation und Anerkennung mehr Bedeutung einräumen. Die bisher ergebnislosen Debatten um abstrakt-generelle Definitionen verlören dadurch an Bedeutung.

${ }^{555}$ Ebd., S. $725 f f$. 


\section{Interdisziplinäre Perspektiven: Konstruktivismus und Anerkennung}

Die Prozesse der Anerkennung kollektiver Identität und die Fragen nach Definitionen von Indigenität und Ethnizität werden auch in der Kultur- und Politikwissenschaft kritisch reflektiert. Im Folgenden sollen diese Perspektiven auf kollektive Identitäten vorgestellt werden, um anhand der Diskurse, wie Gruppen wissenschaftlich im Kontext ihrer sozialen Wirklichkeit begriffen werden, einen kritischen Blick zurück auf die normative Ausgestaltung der völkerrechtlichen Instrumente substaatlicher Gruppenrechte werfen zu können. Die interdisziplinären Perspektiven bilden die argumentative Basis, um Kritik und weitere Empfehlungen zur Modifizierung bestehender völkerrechtlicher Konzepte und Instrumente begründen zu können.

\section{Konstruierte Identitäten}

Aus einer kulturwissenschaftlichen Perspektive werden kulturell distinkte Gruppen, also ethnische Gruppen, Völker, Indigene, Minderheiten und lokale Gemeinschaften, als kulturelle Identitäten verstanden, die durch einen Prozess situativer Aushandlung, einer Auseinadersetzung mit Gleichheit und Differenz konstruiert werden. ${ }^{556}$ Entscheidend sind dabei nicht sämtliche Unterschiede zwischen Gruppen, sondern nur soziale Signifikanten, die eine Bedeutung zur Unterscheidbarkeit erlangt haben. ${ }^{557}$

The features that are taken into account are not the sum of 'objective' differences, but only those which the actors themselves regard as significant [...]. Some cultural features are used by the actors as signals and emblems of differences, others are ignored, and in some relationships radical differences are played down and denied. 558

Kollektive Identitäten sind soziale Konstrukte, die anhand sozialer Signifikanten ein ,Wir‘ gegenüber den ,Anderen' differenzieren. Dieser Ansatz wird seit den 1960 er Jahren in den Kultur- und Geisteswissenschaften unter dem Begriff des Sozialkonstruktivismus diskutiert und versucht mit dieser Herangehensweise die Mechanismen sozialer Realität zu begreifen. ${ }^{559}$

\footnotetext{
556 Richard Jenkins, Retbinking ethnicity: arguments and explorations, 1997, S. 13; Makkonen, Identity, Difference and Otherness, S. 14-19.

${ }_{557}$ Makkonen, Identity, Difference and Otherness, S. 16.

${ }^{558}$ Fredrik Barth, Ethnic groups and boundaries. The social organization of culture difference, 1969, S. 14.

${ }^{559}$ Jenkins, Rethinking ethnicity, S. 11.
} 
Konstruktivismus zeichnet sich durch einen grundlegend neuen Ansatz zur Beschreibung von Kultur und kulturellen Identitäten aus, der sich fundamental von den bis dahin vorherrschenden normativen Kulturtheorien absetzt. Mit einer paradigmatischen Verschiebung der Wahrnehmung wird die Wirklichkeit nunmehr als alleiniges Ergebnis sozial eingebetteter Konstruktions-, Adaptions- und Aushandlungsprozesse begriffen. Für kollektive Identitäten von Minderheiten, indigenen Völkern oder lokalen Gemeinschaften bedeutet dies, dass sie nicht mehr statisch anhand kriterialer Definitionen bestimmt werden können. Ihr Entstehen, ihre Existenz und ihre Auflösung sind vielmehr in spezifischen Kontexten politischer, sozialer und wirtschaftlicher Strukturen für eine wissenschaftliche Analyse greifbar zu machen. Die kollektive Identität einer Gruppe ergibt sich erst durch einen Prozess der Auseinandersetzung, welcher sich als Dialektik von Gleichheit und Verschiedenheit im größeren sozialen Feld und in Form einer Abgrenzung zu ,den Anderen' abspielt. ${ }^{560}$ Kollektive Identität kann sich nicht unilateral entwickeln, sondern bedarf eines Auseinandersetzungsprozesses mit ,den Anderen'. Eine Minderheit bestimmt sich in ihrer kollektiven Identität immer nur gegenüber einer Mehrheit. ${ }^{561}$ Ein indigenes Volk definiert sich nur gegenüber den Kolonisatoren oder einer unterdrückenden, machtvollen Gesellschaft. Die traditionelle Lebensweise einer lokalen Gemeinschaft lässt sich nur im Verhältnis zu einer Gruppe mit ,moderner Lebensweise‘ abgrenzen.

Dieses relationale Verständnis kollektiver Identität wendet sich ausdrücklich gegen Definitionsansätze von Gruppen anhand essentialisierender Merkmale und Kategorien, welche - ähnlich den völkerrechtlichen Definitionsansätzen - verschiedene Kriterien zur Identifizierung kollektiver Identitäten heranziehen. ${ }^{562} \mathrm{Zu}$ nennen wären hier zum Beispiel die Kriterien der historischen Verbindung zu präkolonialen Gesellschaften für indigene Gruppen, ihre aktuelle Erfahrung der Marginalisierung oder eine romantisierende Zuschreibung einer ökologischen, spirituellen und nachhaltigen Lebensweise. Solche Kriterien werden den sich stetig wandelnden und im Fluss befindlichen kulturellen Identitäten nicht gerecht. ${ }^{563} \mathrm{Die}$ Kultur einer Gruppe steht immer in Aushandlung mit den gegebenen Umständen, verändert sich im Austausch mit Anderen und in Reaktion auf innere und äußere Einflüsse. Statische, kriteriale Definitionen von bestimmten Kulturen werden somit als essentialisierend und rassistisch zurückgewiesen. ${ }^{564}$ Kollektive Identitäten sind somit immer in ihrem jeweiligen sozialen, wirtschaftlichen und politischen Kontext zu beurteilen, in dem sie ausgehandelt werden.

\footnotetext{
${ }^{560}$ Ebd., S. 3-25.

561 Ebd.

562 Makkonen, Identity, Difference and Otherness, S. 20.

563 Bedorf, Verkennende Anerkennung, S. 117.

${ }^{564}$ Jenkins, Rethinking ethnicity, S. 9; Makkonen, Identity, Difference and Otherness, S. $42 \mathrm{ff}$.
} 
II. Die Aushandlung kollektiver Identitäten und das Anerkennen

In der politischen Theorie wird kollektive Identität ebenso wie in der Kulturwissenschaft als ein Prozess der Aushandlung, als ein Dialog, verstanden. Dieser Prozess wird unter dem Begriff der ,Anerkennung' diskutiert, wie ihn insbesondere Charles Taylor in seinem Aufsatz ,Die Politik der Anerkennung“ von 1986 prägte. ${ }^{565}$ Im Kontext der Debatten zum Multikulturalismus befasste er sich darin mit der Frage, inwieweit kulturell distinkten Gruppen in einer liberalen Demokratie besondere Rechte und Freiheiten eingeräumt werden sollten sowie mit der ,weitergehenden Forderung [...], dass wir alle die Gleichwertigkeit unterschiedlicher Kulturen erkennen sollen; dass wir sie nicht nur leben lassen, sondern auch ihren Wert anerkennen sollen. "566

Charles Taylor vertritt die These, dass Identität einer Anerkennung bedürfe, da heute die alten Rollenverständnisse überkommen seien. ${ }^{567}$ Gaben bislang gesellschaftlich etablierte Rollen grundsätzlich vor, wie man zu sein und zu handeln habe, bedürfe es mit dem zunehmenden Verlust der Normativität bisheriger Gesellschaftsmodelle im Zuge der Postmoderne Prozessen der Aushandlung und Anerkennung eigener Identität. ${ }^{568}$ Eine individuelle Eigenart der Persönlichkeit könne nicht mehr aus der Gesellschaft und aus Rollenbildern abgeleitet werden, sondern bedürfe der individuellen Anerkennung. 569

Auch für Kollektive, so führt Charles Taylor weiter aus, bedürfe es der Anerkennung, da sie wie Individuen im Dialog mit anderen Gruppen stünden und dadurch ihre Identität stets aushandeln müssten. Gruppen entwickelten demnach erst durch diese dialogische Auseinandersetzung mit den signifikanten Anderen ihre eigene Identität. ${ }^{770}$ Die politikwissenschaftliche Betrachtungsweise begreift den Prozess der Anerkennung somit als notwendigen Teil der Genese und Entwicklung von kollektiver Identität.

Durch die Dekonstruktion festgeschriebener kollektiver Identitäten stellt sich die Frage nach einer Auseinandersetzung mit und einer Einordnung von anderen Identitäten und Kulturen, also die Frage, ob diese unsere Anerkennung erhalten sollen oder nicht. Die Auffassung, dass sich kollektive Identität erst durch einen „offenen Dialog ohne gesellschaftlich vorab festgelegtes Drehbuch“ entwickelt, führt dann zu einer „Politik der gleichheitlichen Anerkennung“; einer Politik, der die Ausgangshypothese zugrunde liegt, dass alle Kulturen von gleichem Wert

\footnotetext{
565 Charles Taylor, Multikulturalismus und die Politik, der Anerkennung, 2009, S. 11-66.

566 Ebd., S. 50.

567 Ebd., S. 19.

568 Zur Weiterentwicklung der „Intersubjektivistischen Anerkennung“ siehe: Axel Honneth, Kampf um Anerkennung: Zur moralischen Grammatik sozialer Konflikte, 2010.

569 Taylor, Multikulturalismus und die Politik der Anerkennung, S. 14-19.

${ }^{570}$ Ebd., S. 20.
} 
seien. ${ }^{571}$ Diese Annahme muss dabei eine Hypothese bleiben, die durch eine ergebnisoffene Beschäftigung mit der anderen Kultur im Einzelfall zu bestätigen oder abzulehnen ist. ${ }^{572}$ Es wäre scheinheilig, stünde das Ergebnis des Anerkennungsprozesses und die Frage nach dem Wert unterschiedlicher Kulturen bereits im Vorhinein fest. „Vielleicht könnte man es auch so formulieren: es wäre ein Anzeichen höchster Arroganz", die Möglichkeit von vornherein auszuschließen, dass andere Kulturen etwas aufweisen, das wir verabscheuen und ablehnen müssen. 573

Neu ist also, dass die Forderung nach Anerkennung heute ausdrücklich formuliert wird. Und dazu kam es auf jene Weise, die ich oben beschrieben habe: indem sich die Vorstellung verbreitete, dass wir durch Anerkennung geformt werden. ${ }^{574}$

In Auseinandersetzung mit Charles Taylor entwickelt Thomas Bedorf diese politische Theorie kollektiver Identitäten dahingehend weiter, dass er den Prozess der Anerkennung von Identität immer als eine nur, verkennende Anerkennung ${ }^{6}$ begreift. ${ }^{575}$ Er erarbeitet für dieses Argument eine detaillierte Analyse des Prozesses der Anerkennung und unterscheidet dazu zwischen dem bloßen Erkennen eines Anderen und dem Anerkennen. ${ }^{576}$ Das Erkennen des Anderen, als erster Schritt hin zum Anerkennen, bedarf einer Zuschreibung von gewissen Eigenschaften, um die Differenzierung vom Selbst zu ermöglichen. Erst durch diese Differenzierung und die Zuschreibung von Eigenschaften der Andersheit vom Selbst, ergibt sich ein Anerkennen. So entwickelt sich aus dem bloßen Erkennen ein Anerkennen einer bestimmten Identität mit bestimmten Eigenschaften. Diese Zuschreibungen der Differenz können dabei nie die wirkliche Identität des Anderen erfassen, so dass ein Anerkennen immer zu einem gewissen Grad verkennende Anerkennung bleibt. ${ }^{577}$ Es entwickeln sich kulturelle Identitäten demnach in konkreten Einzelfällen durch eine Auseinandersetzung und im gegenseitigen Anerkennen, wobei die Eigenschaften der kulturellen Distinktion im Prozess der Identifizierung erst festgestellt und explizit gemacht werden.

\footnotetext{
571 Ebd., S. 23, $50 \mathrm{ff}$.

572 Ebd., S. $55 f$.

573 Ebd., S. 59.

574 Ebd., S. 51.

575 Bedorf, Verkennende Anerkennung.

576 Ebd., S. 118-149.

577 Ebd., S. 149.
} 
III. Die Wirkmächtigkeit der Zuschreibungen des Völkerrechts

Die Genese kollektiver Identität, in Form individueller dialogischer Anerkennungsprozesse, impliziert Zuschreibungen von kriterial greifbaren Eigenschaften, die der Identität einer Gruppen zu eigen sein sollen. Der Dialog der Anerkennung mit der damit einhergehenden Zuschreibung ist jedoch kein responsiver Prozess, bei dem die eigenen Vorstellungen der Ausgestaltung eigener Identität zur Disposition stehen, um voll oder auch nur zu einem gewissen Grad anerkannt zu werden. ${ }^{578}$ Vielmehr ergeben sich die Zuschreibungen über die konkreten Eigenschaften der Identität aus der Vorstellung des Anerkennenden über, den Anderen'. Die populistische Form von solchen Zuschreibungen sind Stereotype, die als Vereinfachung der Vorstellung über ,den Anderen' und somit der sozialen Kategorisierung dienen. ${ }^{579}$ Gegenstand der Aushandlung kollektiver Identitäten sind also nicht das eigene Bild vom Selbst, sondern die Projektionen eines Dritten.

Die Dreistelligkeit der Anerkennung bedeutet, dass sich stets ein Riss im Selbstverhältnis auftut, der nicht $\mathrm{zu}$ schließen ist. Derjenige, als der ich anerkannt werden kann, bin ich ebensowenig, wie derjenige, der ich bin [...]. Ohne das Dazwischentreten eines Dritten als Anerkennungsmedium ist auch das Selbstverhältnis nicht zu haben. ${ }^{580}$

Die konstitutive Bedeutung der Anerkennung für die eigene (kollektive) Identität hat zur Folge, dass Gruppen in diesem Prozess „,wirklichen Schaden nehmen [können], eine wirkliche Deformation erleiden [...], wenn die Umgebung oder die Gesellschaft ein einschränkendes, herabwürdigendes oder verächtliches Bild ihrer selbst zurückspiegelt." 581 Insbesondere das Bild, welches die Mehrheitsgesellschaft von substaatlichen Gruppen hat, ist für Letztgenannte von existenzieller Bedeutung. Dies liegt daran, dass die dominierende Gruppe der Mehrheit im Staat den Minderheiten, indigenen Völkern oder lokalen Gemeinschaften im Allgemeinen als signifikante Andere gegenübersteht. ${ }^{582}$

Im Völkerrecht konkretisieren sich Projektionen und Zuschreibungen kollektiver Identitäten in den positivrechtlichen Strukturen und Konzepten der Rechtsbegriffe substaatlicher Gruppen. Die dargestellten Definitionsansätze beschreiben, was eine Minderheit, ein indigenes Volk und lokale Gemeinschaft ausmachen soll. In diesem Zusammenhang machte bereits Charles Taylor deutlich, dass besonders die liberalen Gesellschaften des Westens wegen ihrer kolonialen Vergangenheit

\footnotetext{
578 Ebd., S. 105, 124.

579 Makkonen, Identity, Difference and Otherness, S. $17 \mathrm{f}$.

580 Bedorf, Verkennende Anerkennung, S. 125.

581 Taylor, Multikulturalismus und die Politike der Anerkennung, S. 13.

582 Bedorf, Verkennende Anerkennung, S. 104; Makkonen, Identity, Difference and Otherness, S. 32.
} 
und einer Marginalisierung von Gruppen ihrer Bevölkerung, die anderen Kulturen entstammen, einer „,angeblichen Überlegenheit“ als schuldig gelten. ${ }^{583}$ Diese schädigende Verkennung beziehungsweise Nicht-Anerkennung, die aus der , angeblichen Überlegenheit' resultiert, sei eine der mächtigsten Waffen der Kolonisatoren, die den von ihnen Unterworfenen ihr eigenes Bild vom Kolonisierten aufprägte. ${ }^{584}$ Die Projektionen und Zuschreibungen, die zur Disposition stehen, um anerkannt zu werden, wirken auf die kollektive Identität ein und bestimmen somit grundlegend das Selbstverständnis einer Gruppe.

Dass es sich bei den völkerrechtlichen Konzepten und Definitionsansätzen aus rechtlicher Perspektive eigentlich nur um die Voraussetzung eines juristischen Begriffs handelt, schließt ihre Wirkmächtigkeit auf kollektive Identitäten dabei nicht aus. Die ihnen inhärenten Bilder und Zuschreibungen wirken nämlich nicht erst auf Gruppen zurück, wenn diese die notwendigen Definitionsmerkmale bereits erfüllen. Vielmehr nehmen die Bilder und Zuschreibungen der völkerrechtlichen Gruppenkonzepte als Teil des öffentlichen Diskurses bereits im Prozess der Konstituierung auf die Konstruktionen kollektiver Identität Einfluss. Das Argument, die Konzepte substaatlicher Gruppen wirkten nur auf solche Gruppen ein, welche die entsprechenden Voraussetzungen erfüllen, ist in der sozialen Wirklichkeit somit nicht aufrecht zu erhalten.

Die bestehenden völkerrechtlichen Konzepte dienen den Prozessen der Aushandlung und Anerkennung kollektiver Identitäten als inhaltlicher Bezugspunkt, wenn politische Ansprüche von Gruppen auf Minderheitenschutz oder Selbstbestimmung geltend gemacht werden. ${ }^{585}$ Die mit den Konzepten verknüpften Rechte befähigen substaatliche Gruppen, sich entgegen einer assimilativen Politik oder vor dem Hintergrund historischen Unrechts gegen gegenwärtige Marginalisierung oder Unterdrückung zu positionieren. Gruppen treten somit bereits vor der rechtlichen Anerkennung als ,Minderheit', ,indigenes Volk oder ,lokale Gemeinschaft in einen Aushandlungsprozess bezüglich ihrer Identität. Sie verorten sich in dieser Interaktion mit Anderen in einer Selbstidentifikation im Sinne der bestehenden rechtlichen Konzepte, um dadurch Einfluss auf die Aushandlung ihrer Identitäten zu nehmen. ${ }^{586}$

$\mathrm{Daß}$ sich kulturelle Kollektive als dieses oder jenes anerkennen lassen wollen, ist dann kein Ausdruck einer Identität, die es zu wahren und deren Werte es zu verteidigen gilt, sondern die

\footnotetext{
583 Taylor, Multikulturalismus und die Politik der Anerkennung, S. 50.

${ }^{584}$ Ebd., S. 51; mit Verweis auf Frantz Fanons einflußreiches Werk „Les damnés de la terre“ (Die Verdammten der Erde), 1961.

585 Klenke und Socha, „Emerging Indigeneity - Völkerrechtswissenschaft und ethnologische Praxis subnationaler kultureller Gemeinschaften".

586 Tania Murray Li, „Articulating Indigenous Identity in Indonesia: Resource Politics and the Tribal Slot", Comparative Studies in Society and History, Vol. 42, Nr. 1 (2000), S. 151.
} 
Formulierung eines temporären politischen Ziels, das man sich zu verwirklichen anschickt. Diese Blickänderung könnte helfen, Anerkennungskonflikte als politischen Streit zu sehen, anstatt als ein Krieg der Kulturen, den es nicht gibt, ohne zugleich unterstellen zu müssen, daß alle kulturellen Kollektive friedlich miteinander auskommen könnten. ${ }^{587}$

Der Prozess der Anerkennung kollektiver Identität als politische Positionierung verdeutlicht die Wirkmächtigkeit der Konzepte und Konstruktionen der positivrechtlich verankerten Konzepte. Die vorteilhaften Rechtspositionen der völkerrechtlichen Konzepte substaatlicher Gruppen sind dabei mit ausdifferenzierten Bildern kollektiver Identität verquickt, deren inhärenten Projektionen und Zuschreibungen im Folgenden nachgegangen werden soll.

\section{Stereotype Bilder, indigener Völker}

Die historischen Wurzeln der Konstruktionen der ,indigenen Völker ${ }^{6}$ liegen in der Entdeckung Amerikas. ${ }^{588}$ Das Bild des ,primitiven Wilden', der durch die Kolonisierung zivilisiert werden soll, bestimmte die Perspektive der Kolonialzeit bis in die 1960er Jahre. ${ }^{589}$ Es ist das Bild des Westens, der es sich zur Aufgabe gemacht hatte, indigene Völker zu entwickeln und in die moderne Gesellschaft zu integrieren. Die Europäer gingen damals davon aus, dass die kolonisierten Völker dieses Eingreifens bedürften, um eine zivilisierte und bessere Lebensführung entwickeln zu können. Gleichzeitig entwickelte sich die Idee des ,edlen Wilden', der geeint mit der Natur in kollektivistischen Strukturen eine nachhaltige Lebensweise spiritueller Ausgeglichenheit führt. ${ }^{590}$ Heute ist es besonders dieses Bild, welches eine post-kapitalistische Sehnsucht der Menschen in Industriestaaten widerspiegelt: Indigene verkörpern ein Alternativmodell zu aktuellen Problemen des Klimawandels, der Umweltverschmutzung und dem auf endlichen Ressourcen aufbauenden Entwicklungs- und Modernisierungszwang. ${ }^{591}$ Diese Bilder und Konstruktionen finden sich nicht nur allgemein in öffentlichen Debatten, sondern waren und sind in den einschlägigen wissenschaftlichen Diskursen zu finden ${ }^{592}$ und ebenso im Völkerrecht positivrechtlich verankert. ${ }^{593}$

\footnotetext{
587 Thomas Bedorf, „Interkulturelle Anerkennung, die Verkennung der Identität und der postkoloniale Diskurs", Vortrag auf dem XXI deutschen Kongress für Philosophie ,Lebenswelt und Wissenschaft', 15. September 2008, S. 10.

588 Michel-Rolph Trouillot, Global Transformations: Antbropology and the Modern World, 2003, S. $14 \mathrm{ff}$.

589 Tennant, „Indigenous Peoples, International Institutions, and the International Legal Literature from 1945-1993“, S. 10.

590 Trouillot, Global Transformations, S. $16 \mathrm{ff}$.

591 Tennant, „Indigenous Peoples, International Institutions, and the International Legal Literature from 1945-1993“, S. 20.

592 Trouillot, Global Transformations, S. $18 \mathrm{ff}$.
} 


\section{Romantisierte Ideen ,lokaler Gemeinschaften "}

Der Begriff der ,lokalen Gemeinschaften', wie er im internationalen Umweltrecht geprägt wurde, normiert in seiner positivrechtlichen Verankerung in der CBD eine gefestigte Zuschreibung kollektiver Identität. Die Bezeichnung als ,lokale Gemeinschaft ${ }^{`}$ beschreibt nicht nur Gruppen mit lokaler Verwurzelung, wie dies allein die wörtliche Bedeutung des Begriffs impliziert. Er zeichnet durch den umweltschutzrechtlichen Kontext das Bild einer Gruppe, die ihre lokalen landwirtschaftlichen Ressourcen subsistenzwirtschaftlich nutzt. ${ }^{994}$ Dies ist eine Beschreibung, die, anders als die Konzepte, indigenes Volk oder ,Minderheit', nicht nur Kategorien der Differenzierung öffnet, wonach etwa eine Unterscheidung nach, anderer' Sprache, Religion, Kultur, oder Regierungsform erfolgt. Der konkretisierende Zusatz in Artikel 8(j) CBD ,embodying traditional lifestyles relevant for the conservation and sustainable use of biological diversity " festigt vielmehr das Bild indigener und lokaler Gemeinschaften und die hinter diesem Konzept liegenden Vorstellungen nicht nur als eine Differenzierung einer ,anderen', traditionellen Wirtschaftsweise, sondern gerade als nachhaltigen, Biodiversität bewahrenden, grünen' Lebensstil.

Zusätzlich färbt das Konzept der indigenen Völker auf die Deutung des Begriffs ,lokale Gemeinschaften ‘ ab. So war das Begriffspaar der ,indigenen und lokalen Gemeinschaft' in der $C B D$ zunächst nur ein Kompromiss, um die Verknüpfung mit Ansprüchen auf Selbstbestimmung zu vermeiden. Der Bedeutungsgehalt der Schutzbedürftigkeit dieser Gruppen in ihrer wirtschaftlichen, sozialen und politischen Situation gegenüber einem Assimilationsdruck von Seiten einer dominierenden Mehrheitsgesellschaft wurde dennoch impliziert. Die Narrative einer indigenen Identität wurde so im Zuge der Bildung des Begriffspaars der ,indigenen und lokalen Gemeinschaften' auf das neue Konzept der ,lokalen Gemeinschaft ${ }^{`}$ übertragen. 595 Das Konzept der, indigenen und lokalen Gemeinschaften' verbildlicht somit die Idealvorstellung kleinbäuerlicher Strukturen nachhaltiger Subsistenzwirtschaft, die sich zum Schutz biologischer Vielfalt und trotz ihrer wirtschaftlichen, sozialen und politischen Marginalisierung den multinationalen Nahrungsmittelindustrien widersetzt.

\section{Die Idealvorstellung von, Minderheiten "}

Die kulturwissenschaftlichen Theorien zu konstruierten kulturellen Identitäten und der Blick auf die Problematik von Zuschreibungen, wie sie sich historisch seit der Auseinandersetzung der Europäer mit den kolonisierten Völkern entwi-

${ }^{593}$ Tennant, „Indigenous Peoples, International Institutions, and the International Legal Literature from 1945-1993“, S. 12-37.

${ }^{594}$ Siehe dazu „C. Lokale Gemeinschaften“ auf S. 102.

595 Siehe zu den entsprechenden juristischen Ausführungen „C. II. Unterschiede zwischen indigenous peoples, indigenous communities und local communities“ auf S. 103. 
ckelt haben, finden auch im Kontext nationaler Minderheiten Anwendung. Sie beschreiben die kulturelle Verschiedenheit zwischen einem, Wir ${ }^{6}$ der Mehrheit in einem Staat auf der einen und den ,Anderen' der Minderheit aufgrund unterschiedlicher Sprache, Religion und ethnischer Herkunft auf der anderen Seite. Die Beziehungen einer Gruppe zu einem ,eigentlichen Heimatstaat ${ }^{`}$ begründet dabei ebenso die kollektive Identität wie die gemeinsame Sprache und Religion der Gruppe. Diese Verbindungen von Gruppen zu Drittstaaten ist ein Erbe der Neuordnungen von Staatsgrenzen nach dem Zweiten Weltkrieg in Europa und auch heute noch Grundlage entsprechender Zuschreibungen einer gewissen Mentalität oder kultureller Unterschiedlichkeiten.

Darüber hinaus impliziert das Bild einer Minderheit das mangelnde politische Gewicht der Gruppe im gesamtstaatlichen Kontext. Mögliche Spannungen kultureller Assimilation und Sezessionsbestrebungen sollen durch etwaige Sonderrechte verhindert werden. Durch solche Sonderrechte soll ebenfalls Raum geschaffen werden, um anderen Norm- und Wertvorstellungen entgegen kommen zu können.

\section{Ergebnis: Holistische Konæepte in den völkerrechtlichen Kategorien}

Für die Aushandlung kollektiver Identität bilden die völkerrechtlichen Konzepte substaatlicher Gruppen mit den jeweiligen nationalen und internationalen Prozessen der Anerkennung wichtige institutionelle Strukturen. Dabei limitiert der völkerrechtliche Diskurs die Vielfältigkeit von substaatlichen Identitäten auf die drei positivrechtlich konkretisierten Konzepte ,Minderheit', ,Indigenes Volk ${ }^{6}$ und ,lokale Gemeinschaft'. Diese Konzepte fungieren mit ihren jeweiligen Narrativen und begrifflichen Konkretisierungen als Bezugspunkte und stellen Terminologien internationaler und interkultureller Diskurse bereit. Zwar weisen die drei Konzepte eine gewisse Flexibilität und Interpretationsspielräume auf, da sie positivrechtlich nicht in völkerrechtlichen Primärquellen festgeschrieben sind. Sie haben aber eine hinreichende Konkretisierung und Kategorisierung erfahren, die konkretisierte Bilder von holistischen Konzepten zeichnet. Es geht in den Aushandlungsprozessen somit um eine Auseinandersetzung mit umfassenden Idealvorstellungen von ,Minderheiten', ,indigenen Völkern' oder ,lokalen Gemeinschaften'. Die Anerkennung einer Gruppe, die ihre kulturelle Distinktion nur auf eine oder wenige kulturelle Praxen stützt, ist angesichts der holistischen Anforderungen der Konzepte schwierig. Nur solche Gruppen, die sich durch eine Vielzahl kultureller Praxen im Sinne der Idealvorstellungen von der Mehrheit unterscheiden, können einen gruppenrechtlichen Status artikulieren und Anerkennung im Sinne eines der drei Konzepte erhalten. Ein gruppenrechtlicher Schutz einzelner kultureller Praxen ist im Rahmen der gegenwärtigen völkerrechtlichen Strukturen nur schwerlich möglich.

Hinzu kommt, dass die bestehenden völkerrechtlichen Konzepte in ihrer holistischen Ausgestaltung eine Reihe von problematischen Aspekten verknüpfen 
und eine differenzierte Anwendung nicht möglich ist. Im Zuge der Aushandlung kollektiver Identität in Auseinandersetzung mit den völkerrechtlichen Konzepten ergeben sich Auswirkungen auf die Identitäten der Gruppen, die, auch wenn sie bewusst als politisch gewählte Instrumentalisierung eingesetzt werden, auf die Lebenswirklichkeit ihrer Mitglieder zurückwirken. Die Auswirkungen der Projektionen und Zuschreibungen liegen dabei in den Definitionsansätzen und konkretisierten Voraussetzungen, also beispielsweise der subsistenzwirtschaftlichen Lebensweise von lokalen Gemeinschaften, der Marginalisierung indigener Gruppen oder der zahlenmäßigen und politischen Inferiorität von Minderheiten. Allen Bildern substaatlicher Gruppen sind somit problematische Aspekte, Zuschreibungen und Projektionen von Eigenschaften inhärent: seien dies Implikationen der Marginalisierung oder einer besonderen Schutzbedürftigkeit. Zusätzlich haben sich im Kontext der Konzepte weitere Zuschreibungen wie die der Rückständigkeit, mangelnde Achtung der Menschen- und insbesondere Frauenrechte oder sonst herabwürdigende stereotype Bilder entwickelt. Eines der konstitutiven Merkmale der drei Konzepte substaatlicher Gruppen ist die Einordnung der Gruppen als Subalterne im Kontext des staatlichen Gefüges, welches die bestehenden Machtstrukturen fortschreibt. Die völkerrechtlichen Konzepte substaatlicher Gruppen strukturieren damit Aushandlungsprozesse kollektiver Identität in holistische Bilder der ,Anderen', die einerseits rechtlich vorteilhafte Positionen versprechen und andererseits problematische Projektionen zuschreiben.

Die drei Konzepte genießen im institutionellen Gefüge des internationalen Rechts einen Grad an Verfestigung, der nur schwer zu verändern ist. Die Regelungen des allgemeinen Völkerrechts bedingen, dass völkerrechtliche Rechtsbegriffe, sofern sie eine gewisse Konkretisierung erfahren haben, nur in Ausnahmesituationen eine Neuinterpretation oder Umdeutung erfahren können. Da die bestehenden Konzepte nur bedingt notwendigen Veränderungen unterzogen werden können, müssen neue Begriffe und Konzepte etabliert werden, um neuen Herausforderungen begegnen zu können. Die dargelegte, überlagernde Anwendbarkeit verschiedener Konzepte und Definitionen verdeutlicht, dass die unterschiedlichen völkerrechtlichen Instrumente als neue Konzepte entwickelt wurden, um veränderten Umständen und neuen Herausforderungen gerecht zu werden.

So beweist zwar die globale Indigenenbewegung, dass das Konzept der Indigenität in neuen geographischen Kontexten konzeptuelle Anknüpfungspunkte kollektiver Identität liefert und politisch machtvolle Bedeutung erlangt. Problematisch ist dabei allerdings, dass durch die Bezüge zu den bestehenden Diskursen die damit verknüpften Zuschreibungen und Projektionen weitergetragen und soziale Problemfelder überblendet werden. ${ }^{596}$ Die Konzeption der ,lokalen Gemeinschaft ${ }^{6}$ im Umweltvölkerrecht musste als Begriff neu entwickelt worden, um den Heraus-

\footnotetext{
${ }^{596}$ Klenke und Socha, „Emerging Indigeneity - Völkerrechtswissenschaft und ethnologische Praxis
} subnationaler kultureller Gemeinschaften“. 
forderungen des Umweltschutzes begegnen zu können. Eine globale Zustimmung konnte nur erreicht werden, indem die Implikationen des Anspruchs auf Selbstbestimmung des bisher vorherrschenden Konzeptes indigener Völker mit Hilfe dieser neuen Begrifflichkeit umgangen wurden. ${ }^{597}$ Die Beispiele verdeutlichen, dass die starren holistischen völkerrechtlichen Konzepte substaatlicher Gruppen nicht die notwendige Flexibilität für Uminterpretationen und Weiterentwicklungen aufweisen und somit problematische Zuschreibungen in geographischer und zeitlicher Hinsicht übertragen und weiter transportiert werden.

\section{Völkerrechtliche Strukturen und Definitionshoheit}

Die Autoren der völkerrechtlichen Gruppenkonzepte und der jeweils damit einhergehenden Zuschreibungen und Projektionen sind grundsätzlich nicht die substaatlichen Gruppen selbst, sondern die Diplomaten und Vertreter der Staaten. In den völkerrechtlichen Strukturen ist die Autorenschaft der Bilder, Projektionen und der Vorstellungen über die ,Anderen' dahingehend institutionell gefestigt, dass substaatliche Gruppen nicht unmittelbar selbst die Konzepte verhandeln, unter denen sie rechtlich eingeordnet und anerkannt werden. Die Ausgestaltung der Konzepte ,Indigenität', ,Minderheit' und ,lokale Gemeinschaft' sind kein unmittelbarer Ausdruck des eigenen Bildes dieser Gruppen.

Die zunehmende Präsenz von Vertretern indigener Völker auf internationaler Ebene begegnet dieser bisher mangelnden Repräsentation. Mittlerweile nimmt eine Vielzahl von Vertretern indigener Gemeinschaften selbst an internationalen Verhandlungen teil. Eine ihrer Kernforderungen dabei ist beispielsweise als ,Völ$\mathrm{ker}^{r}$ im Sinne des Artikel $1 \mathrm{IPbpR}$ anerkannt zu werden. Indigene verstehen sich nicht als substaatliche Gruppen, als Minderheit, untergeordnet in einem staatlichen Gefüge. Vielmehr begreifen sie sich als Völker im Sinne der europäischen Völkerrechtsarchitektur, als tragende Elemente dessen, was ein Völkerrechtssubjekt ausmacht. Diese Forderung ist jedoch nicht zwingend Ausdruck von Sezessionsbestrebungen und dem Willen nach eigener Staatsgründung, wie dies aus einer völkerrechtlichen Lesart der Selbstidentifikation als, $V_{\text {olk }}{ }^{6} \mathrm{zu}$ verstehen wäre. Vielmehr ist dies der Versuch, der Zuschreibung einer subalternen Position im staatlichen Gefüge entgegenzutreten. Es ist Ausdruck eines Selbstverständnisses der Gleichrangigkeit und Ebenbürtigkeit gegenüber dem Staatsvolk und ihren staatlichen Regierungen.

Indigene Völker artikulieren zunehmend ein Selbstverständnis, das den stereotypen Bildern widerspricht, die hinter den klassischen völkerrechtlichen Konzepten von Indigenität liegen. Dies verdeutlicht, dass die starren Konzepte den sich wandelnden Kulturen nicht gerecht werden. Die Vorstellung, man könne indigene

\footnotetext{
597 von Hahn, Traditionelles Wissen indigener und lokaler Gemeinschaften zwischen geistigen Eigentumsrecbten und der ,public domain", S. 33.
} 
Völker anhand ihrer Sprache, Kleidung, geographischer Verwurzelung, Regierungs- und Wirtschaftsform unter eine bestimmte Definition subsumieren, wird heutiger indigener Kultur, die sich in urbanen Kontexten findet, wo Indigene auch Englisch, Französisch und andere Sprachen als Muttersprache sprechen und einer Arbeit in Form eines, westlichen' Berufsalltags nachgehen, nicht gerecht.

Dahingehend wurde die Streichung eines Artikels zur Definition, indigener Völker' in der UNDRIPs von 2007 auch als positiv bewertet. ${ }^{598}$ Eine Deklaration ohne feste Definition eröffnet Spielräume, um eigene Interpretationen und Auslegungen von Indigenität verhandeln zu können. Ohne Definition anhand essentialisierender Kriterien öffnet sich ein gewisser Spielraum für die Vertreter der Gruppen im politischen Diskurs, das Bild und die Zuschreibungen indigener Identität in ihrer aktuellen Ausgestaltung selbst und auf die konkreten Umstände angepasst auszuhandeln.

Trotz positiver Entwicklungen zur Beteiligung von Vertretern der substaatlichen Gruppen bestimmen in den internationalen Prozessen der Aushandlung von Gruppenrechtskonzepten die völkerrechtlichen Strukturen, ob und wie die Beteiligung einer Vielzahl von Akteuren an diesen Diskursen stattfindet. Im Mittelpunkt stehen dabei die Staaten, so dass eine Einflussnahme auf den Bedeutungsgehalt der Konzepte für nichtstaatliche Akteure schwierig ist. Die bestehenden völkerrechtlichen Konzepte kollektiver Identität sind das Ergebnis von Verhandlungsprozessen an denen substaatliche Gruppen selbst nicht oder kaum beteiligt waren. Allerdings, so resümieren Vertreter der politischen Theorie, „sollen Verhandlungen und Übereinkünfte zwischen im Widerstreitet befindlichen Identitäten entscheiden bzw. vermitteln. “599 Die betroffene Gruppe soll in den Aushandlungsprozess ihrer Identität mit einbezogen werden und nicht nur passiver Rezipient von Zuschreibungen sein.

Dies liegt im Wesentlichen daran $[\ldots]$, dass [...] Anerkennung nur dann gewährt werden kann, wenn auch die Mehrheitsgesellschaft die Ansprüche der Minderheit als gerechtfertigt ansieht. Dazu bedarf es einer Integration jener kulturellen Differenzen, die die Minderheit als identitätsstabilisierend ansieht, in das Selbstbild der Mehrheitskultur. Ohne eine für Veränderungen offene Selbstreflexion der Mehrheitsgesellschaft kann eine solche Integration nicht gelingen. 600

Es zeigt sich hier eine notwendige Voraussetzung für einen Anerkennungsprozess. Es bedarf zur Konstituierung kollektiver Identität eines Aushandlungspro-

\footnotetext{
598 Montes und Cisneros, „The United Nations Declaration on the Rights of Indigenous Peoples: the Foundation of a New Relationship between Indigenous Peoples, States and Societies", S. 152. 599 Bedorf, Verkennende Anerkennung, S. 113. ${ }^{600}$ Ebd.
} 
zesses, in den beide Gruppen - Mehrheit und Minderheit - involviert sein müssen, um gegenseitige Anerkennung stiften zu können. Gleichzeitig sollte dieser Prozess dem Machtungleichgewicht zwischen beiden Gruppen entzogen werden, so dass die dominierende Gruppe nicht durch einseitige Zuschreibungen und Projektionen ein Bild der substaatlichen Gruppe festigen kann, welches diese herabwürdigt und verletzt.

\section{Zusammenfassung}

Kollektive Identität wird in den Kulturwissenschaften und in der politischen Theorie als ein Prozess der situativen Aushandlung und Anerkennung verstanden. Anhand sozialer Signifikanten wird in der sozialen Wirklichkeit die Identifizierung der eigenen Gruppe gegenüber den ,Anderen“ im sozialen, wirtschaftlichen und politischen Kontext konstruiert. Unterschiedliche Kulturen können demnach nicht anhand kriterialer Definitionen erfasst werden. Die Beschreibung kollektiver Identität anhand fester Merkmale führt zu Misrepräsentation, würdigt den ,Anderen' herab und ist letztlich rassistisch. Die kulturelle Identität einer Gruppe ist vielmehr Gegenstand eines konstanten Prozesses, eines Dialogs mit dem signifikanten ,Anderen', bei dem kulturelle Differenz fortlaufend neu ausgehandelt wird.

Der Prozess der Aushandlung kollektiver Identität impliziert dabei immer einen Grad verkennender Anerkennung, der sich aus Projektionen und Zuschreibungen ergibt, welche wiederum auf die Gruppe zurückwirken. Dabei können Gruppen ,wirklichen Schaden nehmen, eine wirkliche Deformation erleiden [...], wenn die Umgebung oder die Gesellschaft ein einschränkendes, herabwürdigendes oder verächtliches Bild ihrer selbst zurückspiegelt." "601

In den völkerrechtlichen Konzepten substaatlicher Gruppen manifestieren sich nicht nur besondere Rechte für ,Minderheiten', ,indigene Völker' oder ,lokale Gemeinschaften', sondern auch Vorstellungen und Bilder von diesen ,anderen“ Gruppen, die sich im Laufe ihrer historischen Entwicklung als komplexe, holistische Konstruktionen substaatlicher kollektiver Identität etabliert haben. Einerseits dienen sie als Bezugspunkte nationaler und internationaler Prozesse der Aushandlung und Anerkennung kollektiver Identität, in denen sich Gruppen gegenüber dem dominierenden Teil der Gesellschaft in einem Staat positionieren, um die mit den Konzepten einhergehenden rechtlichen Ansprüche erheben zu können. Andererseits implizieren sie in ihrer jeweiligen holistischen Ausgestaltung eine Stereotypisierung, die bei interkulturellen Konflikten substaatliche kollektive Identitäten nur mit einem unscharfen und meist falschen Bild von Zuschreibungen und Projektionen erfasst.

${ }^{601}$ Taylor, Multikulturalismus und die Politik der Anerkennung, S. 13. 


\section{Schlussfolgerungen}

Mit Hilfe der hier gezeichneten Perspektive der Anerkennung zeigt sich die gegenwärtige Praxis der Anwendung der drei Konzepte substaatlicher Gruppen ,Minderheiten', ,indigene Völker' und ,lokale Gemeinschaften'. Die Perspektive der Anerkennung dient als neuer Zugang zur Beantwortung der Ausgangsfrage der vorliegenden Arbeit: Wie lassen sich kulturell distinkte kollektive Identitäten rechtlich bestimmen, um die völkerrechtlichen Konzepte substaatlicher Gruppen zur Anwendung zu bringen? Diese Frage stellt sich angesichts der Definitionsdebatten für jedes der drei Konzepte, die bisher keinen weltweiten Konsens erreichen konnten und die Bestimmung substaatlicher Gruppen politischen Erwägungen überließ. Durch die Perspektive der Anerkennung lassen sich sowohl die Prozesse als auch der Rechtsakt der Statusverleihung rechtlich greifen und analysieren.

\section{Die Anerkennung sollte die Definitionen ersetzen}

Betrachtet man die gegenwärtige Praxis der Anwendung der Konzepte substaatlicher Gruppen, zeigt sich, dass der Anerkennung für den Rechtsstatus der Gruppen konstitutive Wirkung zukommt. Diese rechtliche Bewertung ergibt sich aus dem Vergleich der Anerkennung substaatlicher Gruppen mit der völkerrechtlichen Staatenanerkennung. Im Gegensatz zur Anerkennung von Staaten, bei der es um die Aufnahme eines neuen Völkerrechtssubjekts in den Kreis der Staatengemeinschaft geht, stehen substaatliche Gruppen gegenüber ihren Heimatstaaten in einem Über-/Unterordnungsverhältnis. Der Rechtsstatus substaatlicher Gruppen ergibt sich innerstaatlich im Rahmen der staatlichen Souveränität und gegenüber anderen Akteuren auf internationaler Ebene im Verhältnis der jeweiligen rechtlichen Beziehung zwischen ihnen. Mit einer notwendigen Anerkennung seitens der Heimatstaaten substaatlicher Gruppen oder anderer Akteure auf internationaler Ebene lassen sich somit die Rechtsbeziehungen einer Gruppe im Einzelfall bestimmen. Die Verknüpfungen auf internationaler Ebene, die sich durch unterschiedliche Anerkennungsbeziehungen zu einem komplexen Netz entwickeln können, wirken zumindest politisch auf die Anerkennung seitens des Heimatstaates zurück. Eine Anerkennung durch den Heimatstaat einer Gruppe stiftet ihr den Rechtsstatus auch gegenüber allen anderen Akteuren auf internationaler Ebene.

Die Anerkennung wirkt zudem als Pendant zur Selbstidentifikation kultureller Distinktion der Gruppen, die in den Definitionsdebatten als Schlüssel zur Bestimmung substaatlicher Gruppen gilt. Für sich genommen kann die Selbstidentifikation als einseitiger Rechtsakt nicht zu tragfähigen rechtlichen Lösungen führen, da ihrem möglichen Missbrauch kein regulatives Moment entgegen gehalten werden kann. Gegenwärtig gibt es keine Regeln, die einen Anspruch auf Anerken- 
nung begründen könnten. Die Staaten können substaatlichen Gruppen die Anerkennung und somit ihren Rechtsstatus auch verweigern.

Die gegenwärtige Praxis der Anwendung der Konzepte substaatlicher Gruppen lässt sich mit Hilfe der Perspektive der Anerkennung begreifen und kann sie als Prozesse und Rechtsakte der Statusverleihung mit rechtlich konstitutiver Wirkung einordnen, ohne auf die bisher vorgeschlagenen Definitionen zurückgreifen zu müssen.

Der Exkurs zu den kultur- und politikwissenschaftlichen Ansätzen zum Umgang mit kulturell distinkter, kollektiver Identität zeigte, dass kriteriale Definitionen in der sozialen Wirklichkeit zu Misrepräsentation führen und, wenn sie in ihrer Projektion von verkennenden Zuschreibungen Kulturen herabwürdigen, letztlich rassistisch sind. Kollektive Identität ist im Sinne des Sozialkonstruktivismus als Ausdruck eines ständigen Prozesses der Aushandlung von kultureller Differenz und Gleichheit zu verstehen, der in seinem jeweiligen sozialen, politischen und wirtschaftlichen Kontext zu beurteilen ist. Vor diesem Hintergrund sind die bestehenden völkerrechtlichen Konzepte substaatlicher Gruppen, wie sie sich insbesondere in den bisher diskutierten Definitionsansätzen konkretisieren, als holistische Bilder ,anderer' Kulturen zu verstehen. Mit den völkerrechtlichen Konzepten können rechtliche Positionen bezüglich kultureller Unterschiede nicht für konkrete einzelne Praxen ausgehandelt werden. Das Völkerrecht stellt nur die drei holistischen Konzepte zur Verfügung, an denen sich kulturell distinkte Gruppen messen lassen müssen, um einen gruppenrechtlichen Status erhalten zu können. Diese Vorstellungen kollektiver Identität sind in Form der bisher entwickelten kriterialen Definitionen verfestigt und starr, da sie sich in den verschiedenen völkerrechtlichen Rechtsquellen etabliert haben und sich die gegebenen Strukturen des Völkerrechts nur schwerlich verändern lassen. Die Zuschreibung von verkennenden Merkmalen seitens des signifikanten Gegenübers führt jedoch zu Gewalt an kollektiver Identität. Kriteriale Definitionen substaatlicher Gruppen sollten demnach in ihrer gegenwärtigen holistischen und starren Form überwunden werden.

Die interdisziplinären Ergebnisse legen somit nahe, die Rechte und den Schutz kulturell distinkter, substaatlicher Gruppen nicht anhand von Definitionen zu verrechtlichen. Mangels konsentierter Definitionen hat dieser Ansatz die gegenwärtige Praxis bisher ohnehin politischen Erwägungen überlassen. Die hier vorgestellte Perspektive der konstitutiven Anerkennung substaatlicher Gruppen kann die Anwendung der Konzepte in ihrem Prozess und statusverleihenden Rechtsakt rechtlich beschreiben, ohne auf die Definitionen zurückgreifen zu müssen. Auch wenn in der Praxis weiterhin bestimmte Schablonen am Werk sein werden, was als typische ,Minderheiten', ,indigene Völker' und ,lokale Gemeinschaften' zu verstehen sei, vermag sie es, die Akteursstrukturen und Verantwortlichkeiten zu begreifen, die für eine kritische Analyse der hintergründigen Machtverhältnisse notwendig sind. Die Perspektive der konstitutiven Anerkennung macht die bisher disku- 
tierten Definitionen nicht nur entbehrlich, sie öffnet auch den Blick auf Machtungleichgewichte.

\section{Es bedarf Regelungen zu Institutionen und Prozessen der Anerkennung}

Im Gegensatz zu den Debatten um Definitionen öffnet die Perspektive der Anerkennung den Blick auf den Prozess und die dabei beteiligten Akteure der Anwendung der Konzepte substaatlicher Gruppen. Die Anerkennung substaatlicher Gruppen ist nicht frei von machtpolitischen Kämpfen. Als analytische Perspektive öffnet sie jedoch den Blick für die Machtstrukturen, unter denen substaatliche Gruppen den politischen Gegebenheiten ihrer Heimatstaaten ausgeliefert sind. Die Heimatstaaten stehen den Gruppen meist als Anspruchsgegner gegenüber, wenn sie zweisprachige Schulen finanzieren, Kompetenzen im Steuerrecht übertragen oder die Verfahren zur Einbeziehung von Gruppen zu Fragen über genetische Ressourcen einrichten sollen. In der Praxis zeigt sich, dass die Heimatstaaten der Anerkennung substaatlicher Gruppen daher grundsätzlich defensiv gegenüberstehen. Die Beurteilung, welchen Gruppen ein rechtlicher Status zusteht und welchen nicht, ist gerade in Grenzfällen eine politisch höchst umstrittene Entscheidung.

Zur Lösung dieser Problematik schlug bereits 1996 die Vorsitzende der working group on indigenous populations Erica-Irene A. Daes die Schaffung einer unabhängigen Institution vor, ,which is fair-minded and open to the views of indigenous peoples and governments, so that there is room for the reasonable evolution and regional specificity of the concept of 'indigenous' in practice. "602 Eine von den Staaten unabhängige Instanz, die nicht unmittelbar in das bipolare Verhältnis zwischen der Gruppe und ihrem Heimatstaat eingebunden wäre, ${ }^{603}$ unterläge weniger stark den politischen Einflüssen und könnte in einem transparenten Verfahren, in dem die Gruppe und der Staat als gleichberechtigte Teilnehmer auftreten und für welches die rechtlichen Regelungen zu Fristen, Beweismitteln usw. im Vorhinein feststehen, die Fragen der Anerkennung in einem offenen Prozess aushandeln.

Als historisches Beispiel dafür kann die Mixed Commission des griechischbulgarischen Minderheitenabkommens von 1919 gelten. ${ }^{604}$ Die Mixed Commission sollte als unabhängige internationale Institution die Anerkennung individueller Gemeinden, Dörfer und Individuen ausführen. Auf die Anfrage an den Ständigen internationalen Gerichtshof, wie mit der Anerkennung und Nicht-Anerkennung ein-

\footnotetext{
602 Sub-Commission on Prevention of Discrimination and Protection of Minorities, „Working Paper by the Chairperson - Rapporteur, Mrs. Erica-Irene A. Daes on the concept of ,indigenous people“", UN Doc. E/CN.4/Sub.2/AC.4/1996/2, Abs. 74.

${ }^{603}$ Einen ähnlichen Vorschlag macht auch Roßkopf, Theorie des Selbstbestimmungsrechts und Minderheitenrechts, S. 470-472.

${ }^{604}$ Convention between Greece and Bulgaria Respecting Reciprocal Emigration, 1 LNTS 67, 27. November 1919.
} 
zelner Gemeinden als Teil der Minderheit verfahren werden solle, antwortete dieser in seiner advisory opinion:

The question whether, in deciding on the application of the Convention, a particular community does or does not conform to the conception described above [i.e. being a minority] is a question of fact which it rests with the Mixed Commission to consider having regard to all the circumstances. [...] The existence of communities is a question of fact; it is not a question of law. [...] The question whether, according to local law, a community was or was not recognized as a juridical person, may be of some importance from the point of view of the form which its possession of property took. But the situation of fact is not thereby altered and there is nothing in the provisions of the Convention respecting the property 'belonging to communities' to indicate that only those communities which have been accorded special legal recognition by the local legislation are meant. ${ }^{605}$

Der bilaterale Vertrag zwischen Bulgarien und Griechenland implizierte die Anerkennung der Existenz entsprechender Minderheiten auf ihren jeweiligen Territorien. Für die Anwendung der Minderheitendefinition, die der StIGH zuvor in seiner Entscheidung konkretisierte, ${ }^{606}$ waren jedoch nicht nationale Gesetze entscheidend, sondern die faktische Situation, die durch die Mixed Commission bewertet werden sollte, um Mitglieder der Minderheit individuell anzuerkennen.

Allerdings ist fraglich, ob Staaten der Übertragung der Anerkennungskompetenz auf eine unabhängige internationale Institution zustimmen würden. Ebenso fraglich ist es, die Anerkennungskompetenz auf eine Gruppe benachbarter Staaten oder eine regionale inter- oder supranationale Organisation zu übertragen, wie dies bei neuesten Entwicklungen zur Staatenanerkennung zu beobachten ist. ${ }^{607}$ Einerseits würde solch eine institutionelle Ordnung das Anerkennungsverfahren der oben dargestellten, gegenläufigen Akteurskonstellation und den ihr inhärenten Machtstrukturen entziehen. Andererseits stellen sich bei solch einem Vorschlag die Fragen, inwiefern solch eine Internationalisierung der Anerkennung auch Ansprüchen einer demokratischen Legimitation eines potentiellen Entscheidungsträgers genügen würde und wie eine Beteiligung der substaatlichen Gruppe und der Mehrheitsgesellschaft am Verfahren der Anerkennung aussehen könnte.

\footnotetext{
${ }^{605}$ Permanent Court of International Justice, „Greco-Bulgarian Communities“, 1930 PCIJ, Ser B, No. 17, S. 22.

${ }^{606}$ Siehe dazu „A. I. Definitionsansatz des Ständigen Internationalen Gerichtshof“ auf S. 83.

${ }^{607}$ Saxer, Die internationale Steuerung der Selbstbestimmung und der Staatsentstehung, S. $722 \mathrm{ff}$.
} 
Neben den institutionellen Zuständigkeiten stellen sich also auch Fragen nach völkerrechtlichen Vorgaben de lege ferenda für die Gestaltung der Prozesse der Anerkennung substaatlicher Gruppen. Aus den obigen Darstellungen des Vergleichs zur Staatenanerkennung und den interdisziplinären Perspektiven ergeben sich dafür bedenkenswerte Aspekte. Die Analyse der neueren Praxis der Staatenanerkennung hat gezeigt, dass neben der Bewertung des Vorliegens der Merkmale der Staatlichkeit auch weitergehende Interessen Teil der Anerkennungsprozesse werden. Es werden Vorgaben zur notwendigen demokratischen Verfasstheit, zur Verpflichtung zu menschenrechtlichen Garantien und zur Teilnahme an Verfahren der friedlichen Streitbeilegung gemacht. Diese Aspekte könnten auch für die Anerkennung substaatlicher Gruppen zur Voraussetzung gemacht werden. Denkbar wäre zudem die Vorgabe einer expliziten Regelung und Verwaltung ihrer Mitglieder. Die Notwendigkeit dieser und weiterer Aspekte hängt allerdings vom konkreten Einzelfall ab.

Aus den kultur- und politikwissenschaftlichen Perspektiven ergibt sich insbesondere die Vorgabe der Beteiligung der betroffenen Gruppen bei der Aushandlung ihrer kulturellen Identität. Um den Grad der verkennenden Anerkennung zu reduzieren und sich konstant wandelnden Kulturen gerecht werden zu können, sind darüber hinaus dauerhafte Institutionen und Prozesse eines Dialogs zwischen den Gruppen notwendig. Es bedarf eines Dialogs auf Augenhöhe, in dem die Bilder des jeweils ,Anderen“ konstant verhandelt werden können. Die Anerkennung sollte demnach den politischen Diskursen zwischen den Gruppen nicht entzogen werden. Institutionelle Strukturen der Anerkennung müssten de lege ferenda also sowohl Machtungleichgewichte umgehen, was durch internationale Organisationen gut möglich erscheint, gleichzeitig aber die Beteiligung der Minderheit und der Mehrheit in Form eines konstanten Prozesses der individuellen Aushandlung der Bilder kollektiver Identität sicherstellen. 


\section{Teil 4: Verhandlungen im WIPO Intergovernmental Committee}

Das Intergovernmental Committee der World Intellectual Property Organization zu geistigem Eigentum und genetischen Ressourcen, traditionellem Wissen und traditionellen kulturellen Ausdrucksformen ist Forum neuer Entwicklungen zu völkerrechtlichen Gruppenrechten. Als kollektiv Berechtigte neuer Rechtsinstrumente geistigen Eigentums an traditional knowledge und traditional cultural expressions werden hier für die Normierung der beneficiaries sowohl die bestehenden Konzepte der ,indigenous peoples/communities', ,local communities' und ,minorities' als auch die neuen Begrifflichkeiten ,cultural community 'und ,traditional community "verhandelt.

Diese aktuellen Entwicklungen zur Schaffung neuer Rechte für substaatliche Gruppen sind insbesondere deshalb interessant, weil neue Institutionen zur Anwendung der Gruppenrechte geschaffen werden sollen. Im Sinne der in Teil 3 vorgestellten Perspektive der Anerkennung substaatlicher Gruppen sind die hier diskutierten Institutionen Teil neuer Akteurskonstellationen und Prozesse, welche die Anwendung der Rechte substaatlicher Gruppen grundlegend verändern könnten. 


\section{A. Geschichte und Hintergrund}

Die Schaffung kollektiver Schutzinstrumente geistigen Eigentums für kulturelle Ausdrucksformen war bereits seit den 1960er Jahren, damals unter den Begriffen ,Folklore' sowie ,Ausdrucksformen von Folklore', Thema im Rahmen der UNESCO und WIPO. ${ }^{608}$ Seit 2001 wird der Schutz kultureller Ausdrucksformen nun zusammen mit den Themenkomplexen genetische Ressourcen und traditionelles Wissen im IGC verhandelt. Gemeinsamer Bezugspunkt ist das Recht des geistigen Eigentums. Die beiden letztgenannten Themenbereiche sind historisch vorrangig bei den Entwicklungen des internationalen Umweltrechts zu verorten und werden nun im IGC erstmals gemeinsam mit dem Schutz von kulturellen Ausdrucksformen und Folklore verhandelt. ${ }^{609}$

Inhaltlich lassen sich die Verhandlungen des IGC somit zweiteilen. Auf der einen Seite stehen die Verhandlungen zu genetischen Ressourcen, für die im Kontext des bestehenden Patentrechts um die Entwicklung von Datenbanken und Informationssystemen zur defensive protection sowie um die Offenlegung der Herkunft im Patentanmeldungsverfahren gerungen wird. ${ }^{610}$ Die $C B D$ verknüpfte dazu das sog. assoziierte traditionelle Wissen bisher in einem konkretisierten Verständnis mit den Regelungen zu genetischen Ressourcen. ${ }^{611}$ Auf der anderen Seite stehen die Diskussionen um traditionelles Wissen in seiner umfassenden Bedeutung, die im IGC weitergeführt werden und hier eine inhaltlich parallele Behandlung zum verwandten Bereich der traditionellen kulturellen Ausdrucksformen erfahren. ${ }^{612}$ So verweisen die commentaries der draft articles zu traditionellem Wissen häufig auf entsprechende Diskussionen im Kontext der traditionellen kulturellen

${ }^{608}$ Der Begriff „Folklore“ stand früher im Mittelpunkt der Diskussionen und wurde später durch die Ausdrücke „Expressions of Folklore“ und heute „Traditional Cultural Expressions“ abgelöst. Die Bezeichnung „Folklore“ wurde „als anachronistische Referenz an ein eingefrorenes kulturelles Archiv“ betrachtet. Rosemary J. Coombe, „Der zunehmende Geltungsbereich kultureller Besitztümer und ihrer Politik“, in: Regina Bendix, Stefan Groth, und Kilian Bizer (Hrsg.), Die Konstituierung von Cultural Property: Forschungsperspektiven, 2010, S. 254; Siehe dazu auch: WIPO IGC, „Glossary of Key Terms Related to Intellectual Property and Genetic Resources, Traditional Knowledge and Traditional Cultural Expressions" (Glossar), WI-

PO/GRTKF/IC/20/INF/13, 7. Dezember 2011, Annex, Stichwort „Traditional Cultural Expressions“. Im Folgenden wird also auch hier der Begriff „,traditionelle kulturelle Ausdrucksform" verwendet, sofern nicht auf Begrifflichkeiten in Primärquellen zurückgegriffen wird.

${ }^{609}$ Siehe dazu die Ausführungen unter „C. II. 5. Verhandlungen zu Rechten geistigen Eigentums und genetischen Ressourcen" auf S. 73.

${ }^{610}$ WIPO IGC, „Options for Future Work on Intellectual Property and Genetic Resources“, WIPO/GRTKF/IC/20/5, 10. Oktober 2011.

${ }^{611}$ Siehe dazu „C. III. Regelungen zum Schutz traditionellen Wissens“ auf S. 76.

${ }^{612}$ Die Entwicklungen zu Schutzinstrumenten von TK im weiteren Sinne und TCEs sind so eng miteinander verzahnt, dass im Folgenden gemeinsam auf beide Bereiche eingegangen werden soll. Der Fokus liegt dabei aber auf den Regelungen zu TCEs. 
Ausdrucksformen. ${ }^{613}$ Der Verhandlungsgegenstand des IGC lässt sich somit in die Themenbereiche der genetischen Ressourcen und dem assoziierten traditionellen Wissen auf der einen Seite sowie die Themen traditionelle kulturelle Ausdrucksformen und traditionelles Wissen im weiteren Sinne auf der anderen Seite strukturieren. Für die neuen Regelungen und Rechtsinstrumente bezüglich der Letztgenannten werden im IGC kollektivrechtliche Ansätze verhandelt, die hier näher untersucht werden sollen.

\section{Vorgeschichte}

Die Vorgeschichte des WIPO IGC reicht mit verschiedenen Initiativen zur Schaffung von Schutzrechten für TCEs lange vor die erste Sitzung im Jahr 2001 zurück. Sie verbindet verschiedene Entwicklungen auf nationaler Ebene einiger Staaten und in unterschiedlichen internationalen Kontexten, wo bereits Ansätze eines Kollektivrechts geistigen Eigentums an kulturellen Ausdrucksformen, Folklore und Ausdrucksformen von Folklore diskutiert wurden.

Hintergrund dieser Initiativen ist die zunehmende missbräuchliche Nutzung von kulturellen Ausdrucksformen durch Dritte. Verstärkt durch die zunehmende Technisierung und Globalisierung werden Ausdrucksformen von Folklore kopiert, verbreitet und kommerzialisiert, ohne dass dabei Rücksicht auf die sozialen, kulturellen und ökonomischen Interessen der Gemeinschaften genommen wird, aus denen sie stammen. Um diesen Verletzungshandlungen entgegenzuwirken, entwickelten sich bereits seit Ende der 1960er Jahre im Kontext des Urheberrechts nationaler Gesetzgebung in Entwicklungsländern spezielle Schutzvorschriften für Folklore. ${ }^{614}$ Vergleichbar mit den Auseinandersetzungen um traditionelles Wissen kontextualisieren sich die Spannungen jedoch vor allem im globalen Nord-Süd Konflikt. Auf der einen Seite stehen die Staaten, in denen kulturelle Ausdrucksformen zwar bestimmten Gruppen zugeordnet werden können, aber mangels individueller Autorenschaft internationalrechtlich nicht durch das System des Urheberrechts geschützt sind. Auf der anderen Seite stehen die Staaten, deren Strukturen der Produktion und Verbreitung von kulturellen Ausdrucksformen in Einklang mit den bestehenden nationalen und internationalen Rechten geistigen Eigentums profitable Kulturindustrien geschaffen haben. So nutzen westliche Medienunternehmen die kulturellen Ausdrucksformen, die in ihrer Herkunft im globalen Süden verortet werden können und sich in der public domain befinden, um

\footnotetext{
${ }^{613}$ Siehe dazu die Verweise bei den Regelungen zu beneficiaries und Scope of Protection: WIPO Generalversammlung, „Matters Concerning the Intergovernmental Committee on Intellectual Property and Genetic Resources, Traditional Knowledge and Folklore (IGC)“, WO/GA/40/7, 12. August 2011, Annex B, S. 10 und 14.

${ }^{614}$ Mit einer Aufzählung entsprechender gesetzlicher Initiativen: WIPO, „The Protection of Expressions of Folklore: The Attempts at International Level“, WIPO/ACAD/E/99/12, 1. April 1999, S. 2.
} 
damit wirtschaftliche Gewinne zu erzielen ohne die eigentlichen Träger der kulturellen Ausdrucksformen daran zu beteiligen.

\section{Nationale und internationale Entwicklungen im Kontext des Urbeberrechts}

Auf internationaler Ebene wurden Regelungen zum Schutz von Folklore erstmals 1967 auf der Konferenz in Stockholm diskutiert. In Artikel 15 (4) (a) der Berne Convention, der den Schutz von Ausdrucksformen von Folklore bezwecken soll, 615 findet sich folgende Formulierung:

In the case of unpublished works where the identity of the author is unknown, but where there is every ground to presume that he is a national of a country of the Union, it shall be a matter for legislation in that country to designate the competent authority which shall represent the author and shall be entitled to protect and enforce his rights in the countries of the Union. ${ }^{616}$

In dem von UNESCO und WIPO entwickelten Tunis Model Law von 1976, welches als Vorlage für nationale Urheberrechtsgesetzgebung dienen soll, werden in Artikel 2 (1) (iii) ,works derived from national folklore“ genannt sowie in Artikel 6 explizit Regeln für „Works of national folklore“ getroffen. ${ }^{617}$ Beide Instrumente, Artikel 15 (4) Berne Convention und das Tunis Model Law, bezeichnen dabei eine competent national authority als ausführende Instanz. Mangels einer individuellen Autorenschaft bezüglicher dieser Ausdrucksformen soll über solch eine Institution die Ausübung entsprechender Rechte gewährleistet werden.

Der Schutz von Folklore durch das Instrument des Urheberrechts gestaltet sich jedoch schwierig. Ausdrucksformen von Folklore sind ihrer Natur nach das Resultat eines nicht-individualisierten, fortschreitenden Prozesses von Nachahmung und Imitation kreativer Aktivität, die in einer kulturellen Gemeinschaft ausgeführt wird. Ziel ist es, folkloristische Ausdrucksformen zu bewahren und gleichzeitig zugänglich zu machen. Für die Anwendung des Schutzes durch das Urheberrecht bedarf es hingegen einer gewissen innovativen Schaffenshöhe für ein ,neues Werk', welches einem bestimmten Autor zugerechnet werden können

615 Ebd., S. 4; Agnès Lucas-Schloetter, „Folklore“, in: Silke v. Lewinski (Hrsg.), Indigenous heritage and intellectual property: genetic resources, traditional knowledge and folk:lore, 2008, S. 350; Sam Ricketson und Jane C. Ginsburg, International copyright and neighbouring rights: the Berne Convention and beyond, 2006, S. 513.

616 „Berne Convention for the Protection of Literary and Artistic Works“, 331 UNTS 217, 9. September 1886, Art. 15 (4) (a): „In the case of unpublished works where the identity of the author is unknown, but where there is every ground to presume that he is a national of a country of the Union, it shall be a matter for legislation in that country to designate the competent authority which shall represent the author and shall be entitled to protect and enforce his rights in the countries of the Union."

617 UNESCO/WIPO, Tunis Model Law on Copyright for Developing Countries, 1976. 
muss. ${ }^{618}$ Ziel des copyrights ist hingegen ein ökonomischer Anreiz zur Schaffung neuer Werke. Zwar sind im Tunis Model Law Reglungen getroffen worden, die diesen Problemen Rechnung tragen sollen. Das Spannungsverhältnis der unterschiedlichen Regelungsinteressen des Urheberrechts auf der einen Seite und dem Schutz von Folklore auf der anderen vermag es jedoch nicht zu lösen. ${ }^{619}$ Vor dem Hintergrund dieser grundlegenden Probleme und Zielkonflikte wurden in der nachfolgenden Entwicklung für den Schutz von Ausdrucksformen von Folklore Instrumente sui generis in den Blick genommen.

\section{Model Laws als sui generis Instrumente auf internationale Ebene}

Eine Nachricht Boliviens an den Generaldirektor der UNESCO vom 24. April 1973 initiierte die Thematisierung des Schutzes von Folklore in der Kulturorganisation der Vereinten Nationen. ${ }^{620}$ Bolivien forderte unter anderem ein Zusatzprotokoll zur Konvention vom Schutz von Tonträgern, in dem alle Rechte an kulturellen Ausdrucksformen von Kollektiven oder anonymer Herkunft traditionellen Charakters als Eigentum dem jeweiligen Heimatstaat zuerkannt werden sollten. Der Generaldirektor der UNESCO leitete diese Nachricht an das gemeinsame Gremium des Intergovernmental Copyright Committee und das Executive Committee der Bern Union weiter, welches daraufhin wieder das Sekretariat der UNESCO mit diesem Thema betraute. Diese legte schließlich 1975 eine Studie zur Desirability of providing protection for folklore at the international level vor. ${ }^{621}$

Im Jahr 1978 beschlossen auch die Governing Bodies der WIPO, dass juristische Standards zum Schutz von Folklore nötig seien. Das International Bureau der WIPO erarbeitete daraufhin einen ersten Entwurf eines sui generis Instruments nach Art

618 Zur weiteren Kritik an Instrumenten des geistigen Eigentums im Rahmen der aktuellen Debatte um Cultural Property siehe Michael Fobes Brown, Who owns native culture?, 2004; Coombe, The cultural life of intellectual properties.

${ }^{619}$ Lucas-Schloetter, „Folklore“, S. 446f., die unter anderem den Widerspruch zwischen der im Tunis Model Law angedachten ,zahlenden public domain“ und dem Interesse, Folklore frei zugänglich zu halten, darstellt.

${ }^{620}$ Republic of Bolivia, Ministry of Foreign Affairs and Religion, „Letter to the Director-General of UNESCO“, 24. April 1973, abgedruckt als Annex A: Intergovernmental Copyright Committee, „Proposal for international instrument for the protection of folklore“, 12th session 1973

IGC/XII/12, 1. Oktober 1973. Die Forderung nach einem internationalen Schutz von Folklore geht einer Anekdote nach auf die Empörung des bolivianischen Verteters über eine Reinterpretation des Anden Volksliedes „El Condore Pasa“ von Simon and Garfunkel zurück.

${ }^{621}$ Intergovernmental Committee of the Universal Copyright Convention as Revised at Paris on 24 July 1971 und International Union for the Protection of Literary and Artistic Works. Executive Committee, „Consideration of the possibility of establishing an international instrument for the protection of folklore“, B/EC/IX/11 und IGC/XR.1(1971)15, 10. Dezember 1975, Annex; Sherkin datiert die Erstellung dieses Dokuments fälschlicherweise auf 1971, Samantha Sherkin, „A Historical Study on the Preparation of the 1989 Recommendation on the Safeguarding of Traditional Culture and Folklore“, in: Peter Seitel (Hrsg.), Safeguarding traditional cultures: a global assessment, 2001, S. 44f. 
des Schutzes geistigen Eigentums gegen unberechtigte Nutzung und Entstellung. ${ }^{622}$ Nach gemeinsamen Arbeitsgruppentreffen von WIPO und UNESCO in Genf 1980 und Paris 1981 nahm ein Committee of Intergovernamental Experts 1982 in Genf die Model Provisions for National Laws on the Protection of Expressions of Folklore an. ${ }^{623}$

\section{Entwürfe multilateraler Instrumente}

Bereits während des Treffens der Governmental Experts wurden Stimmen für die Schaffung eines multilateralen Instruments laut, welches den internationalen Schutz von Ausdrucksformen von Folklore über eine reziproke, bilaterale Anerkennung hinaus sicherstellen sollte. ${ }^{624}$ Bei einem Treffen einer Expertengruppe im Dezember 1984 wurde ein entsprechender multilateraler Vertrag diskutiert, der Mitgliedstaaten zur gegenseitigen Anerkennung jeweilig geschützter Ausdrucksformen von Folklore verpflichten würde. Als Diskussionsgrundlage legten UNESCO und WIPO gemeinsam den Draft Treaty for the Protection of Expressions of Folklore Against Illicit Exploitation and other Prejudicial Actions ${ }^{625}$ vor, der grundsätzlich auf den Model Provisions von 1982 aufbaute. Die Verhandlungen verliefen jedoch ohne Erfolg. Es mangele an Erfahrung mit dem Umgang von Instrumenten zum Schutz von Ausdrucksformen von Folklore, so die offizielle Begründung. Insbesondere fehle es an notwendigen Quellen und Archiven zur Bestimmung von Ausdrucksformen von Folklore anderer Länder sowie an Mechanismen, um transnationale Konflikte bei der Bestimmung von überregionalen Ausdrucksformen beilegen zu können. ${ }^{626}$ Daraufhin lehnten 1985 die Mitgliedstaaten beim gemeinsamen Treffen der Berne Convention und des Intergovernmental Committees der Universal Copyright Convention einen multilateralen Vertrag zum Schutz von Ausdrucksformen von Folklore als verfrüht ab. ${ }^{627}$

Mehr als zehn Jahre später empfahlen das Expertenkomitee eines möglichen Zusatzprotokolls zur Berne Convention und das Expertenkomitee eines möglichen Instruments zum Schutz, der Rechte von ausübenden Künstlern und Tonträgerherstellern auf

${ }^{622}$ WIPO, „The Protection of Expressions of Folklore: The Attempts at International Level“, WIPO/ACAD/E/99/12, S. 5.

${ }^{623}$ Ebd.; United Nations Educational, Scientific, and Cultural Organization und World Intellectual Property Organization, „Model Provisions for National Laws on the Protection of Expressions of Folklore Against Illicit Exploitation and other Forms of Prejudicial Action“, 1985.

${ }^{624}$ Eine solche Anerkennung ist beispielsweise in Section 12 der Model Privisions angelegt. WIPO, "The Protection of Expressions of Folklore: The Attempts at International Level“, WIPO/ACAD/E/99/12, S. 12.

${ }^{625}$ Erneut abgedruckt in: WIPO IGC, „Final Report on National Experiences with the Legal Protection of Expressions of Folklore", WIPO/GRTKF/IC/3/10, 25. März 2002, Annex IV.

${ }^{626}$ WIPO, „The Protection of Expressions of Folklore: The Attempts at International Level“, WIPO/ACAD/E/99/12, S. 12.

${ }^{627}$ Lucas-Schloetter, „Folklore“, S. 452; WIPO, „The Protection of Expressions of Folklore: The Attempts at International Level“, WIPO/ACAD/E/99/12, S. 13. 
ihrer gemeinsamen Sitzung 1996 die Gründung eines internationalen Forums, welches sich mit dem Erhalt und Schutz von Ausdrucksformen von Folklore, Aspekten geistigen Eigentums und Folklore sowie mit der Harmonisierung von regionalen Interessen befassen solle.628 Dieser Empfehlung waren entsprechende regionale Treffen und bereits eine Einladung Thailands, ein entsprechendes Forum zu veranstalten, vorausgegangen. ${ }^{629}$ Vom 8. bis zum 10. April 1997 wurde daraufhin in Zusammenarbeit von WIPO und UNESCO das UNESCO/WIPO World Forum on the Protection of Folklore in Phuket abgehalten. Der dort angenommene Phuket Plan of Action sah vor, dass nach regionalen Beratungstreffen ein Expertenkomitee ein internationales Abkommen zum sui generis Schutz von Folklore entwickeln soll. Dieses Abkommen solle möglichst bei einem Treffen von Staatenvertretern in der zweiten Hälfte des Jahres 1998 angenommen werden. ${ }^{630}$ Ausgehend vom World Forum in Phuket ergriff WIPO daraufhin zahlreiche Initiativen: neun ,fact-finding missions' in den Jahren 1998 und 1999 zu den Bedürfnissen und Erwartungen von Trägern traditionellen Wissens ${ }^{631}$; zwei runde Tische in Genf632; sowie vier regional consultations in den Regionen Afrika ${ }^{633}$, Asien/Pazifik ${ }^{634}$, Arabische Länder ${ }^{635}$ sowie Lateinamerika und Karibik ${ }^{636}$. Drei dieser regionalen Konsultationen sprachen sich dabei für die Gründung eines eigenständigen Komitees im Rahmen von WIPO aus, welches sich mit den Themen Folklore und traditionelles Wissen befassen sollte. Daraufhin wurde in der 26. Sitzung der WIPO Generalversammlung vom 25. September bis zum 3. Oktober 2000 auf Initiative

${ }^{628}$ WIPO, Committee of Experts to a Possible Protocol to the Berne Convention und Committee of Experts on a Possible Instrument for the Protection of the Rights of Performers and Producers of Phonograms, „Report adopted by the committees“, BCP/CE/VI/16 - INR/CE/V/14, 9. Februar 1996, S. 269.

${ }^{629}$ WIPO, ,The Protection of Expressions of Folklore: The Attempts at International Level“, WIPO/ACAD/E/99/12, S. 15.

${ }^{630}$ WIPO, „The Protection of Expressions of Folklore: The Attempts at International Level“, Intellectual Property in Asia and the Pacific, Nr. 56/57 (1998). Die Vertreter des Vereinigten Königreiches und der Vereinigten Staaten von Amerika identifizierten sich nicht mit dem verabschiedeten Phuket Plan of Action.

${ }^{631}$ WIPO, Intellectual Property Needs and Expectations of Traditional Knowledge Holders, WIPO Report on Fact-finding Missions on Intellectual Property and Traditional Knowledge (1998-1999), 2001.

632 WIPO, „Roundtable on Intellectual Property and Indigenous Peoples“, WIPO/INDIP/RT/98, 23.-24. Juli 1998; WIPO, „Roundtable on Intellectual Property and Traditional Knowledge“, WIPO/IPTK/RT/99, 1.-2. November 1999.

${ }_{633}$ WIPO, „WIPO-UNESCO African Regional Consultation on the Protection of Expressions of Folklore“, WIPO-UNESCO/FOLK/AFR/99, 23.-25 März 1999.

${ }^{634}$ WIPO, ,WIPO-UNESCO Regional Consultation on the Protection of Expressions of Folklore for Countries of Asia and the Pacific", WIPO-UNESCO/FOLK/ASIA/99, 21.-23. April 1999

${ }^{635}$ WIPO, , WIPO-UNESCO Regional Consultation on the Protection of Expressions of Folklore for Arab Countries“, WIPO-UNESCO/FOLK/ARAB/99, 25.-27. Mai 1999.

${ }^{636}$ WIPO, „WIPO-UNESCO Regional Consultation on the Protection of Expressions of Folklore for Latin America and the Caribbean“, WIPO-UNESCO/FOLK/LAC/99, 14.-16. Juni 1999. 
der afrikanischen Staaten das Intergovernmental Committee on Intellectual Property and Genetic Resources, Traditional Knowledge and Folklore (IGC) ins Leben gerufen. ${ }^{637}$

\section{Mandat des Intergovernmental Committee}

Das Mandat des Intergovernmental Committee on Intellectual Property and Genetic Resources, Traditional Knowledge and Folklore, welches ihm für je 2 Jahre von der WIPO Generalversammlung gegeben wird, entwickelte sich seit Beginn der Verhandlungen von der recht offenen Formulierung

informal consultations [... on] intellectual property issues that arise in the context of: (i) access to genetic resources and benefit sharing; (ii) protection of traditional knowledge, innovations and creativity, whether or not associated with those resources; and (iii) the protection of expressions of folklore, including handicrafts ${ }^{638}$

zu einem zielgerichteteren Mandat der

text-based negotiations with the objective of reaching agreement on a text of an international legal instrument (or instruments) which will ensure the effective protection of GRs, TK and TCEs. ${ }^{639}$

Im Vergleich zu der 1997 in Phuket angedachten Konferenz von Staatenvertretern für ein von Experten ausgearbeitetes sui generis Instrument zum Schutz von Folklore für die zweite Jahreshälfte 1998 lässt sich diese Konkretisierung des Mandats über einen Zeitraum von über 10 Jahren als recht zögerlich bewerten. Der Grund für das langsame Fortschreiten der Verhandlungen erschließt sich allerdings anhand einer Gesamtbetrachtung der gegenläufigen Interessen der beteiligten Staaten. ${ }^{640}$

${ }_{637}$ WIPO Generalversammlung, ,"Twenty-Sixth (12th Extraordinary) Session Geneva, September 25 to October 3, 2000, Report", WO/GA/26/10, 3. Oktober 2000, Abs. 27-71; WIPO Generalversammlung, „Matters Concerning Intellectual Property and Genetic Resources, Traditional Knowledge and Folklore“, WO/GA/26/6, 25. August 2000, Abs. 13, 16-18; Lucas-Schloetter, „Folklore“, S. 339.

${ }^{6} 38$ WIPO Generalversammlung, „Matters Concerning Intellectual Property and Genetic Resources, Traditional Knowledge and Folklore“, WO/GA/26/6, Abs. 20.

639 WIPO Generalversammlung, „Matters Concerning the Intergovernmental Committee on Intellectual Property and Genetic Resources, Traditional Knowledge and Folklore (IGC)“, WO/GA/40/7, Abs. 16 (a).

${ }^{640}$ Nähre Ausführungen zu den komplexen Verhandlungen und eine Analyse des langsamen, aber stetigen Fortschritts im Rahmen des IGC findet sich in: Stoll und von Hahn, „Indigenous peoples, indigenous knowledge and indigenous resources in international law"; Matthias Lankau und Kilian Bizer, „Die verborgene Effektivität minimaler Resultate in internationalen Verhandlun- 
III. Verlauf der Verhandlungen des Intergovernmental Committee

Der Verlauf der Verhandlungen im IGC weist neben den halbjährlich stattfindenden regulären Sitzungen sechs Stränge von Aktivitäten auf: Fragebögen zu nationalen Erfahrungen, die Draft Provisions inklusive dreier Kommentierungsphasen, Kommentaren zur List of Key Issues, die Gap Analysis, Intersessional Workinggroups mit je einer Sitzung zu den drei Themenbereichen sowie ein Glossar, welches die wichtigsten verwendeten Begrifflichkeiten erklärt.

\section{Fragebögen zu nationalen Erfahrungen}

Bereits in der 1. Sitzung des IGC wurde das Sekretariat mit der Sammlung von Informationen zu nationalen Erfahrungen mit Rechtsinstrumenten zum Schutz von genetischen Ressourcen, traditionellem Wissen und Folklore beauftragt. ${ }^{641}$ Das Sekretariat erstellte daraufhin verschiedene Fragebögen zu bestehenden nationalen Schutzmechanismen geistigen Eigentums für traditionelles Wissen, zu nationalen Regelungen der Vertragsgestaltung im Bereich des geistigen Eigentums, zum Zugang, benefit sharing und den Voraussetzungen der Offenlegung bei genetischen Ressourcen und traditionellem Wissen im Patentverfahren, zu Datenbanken von traditionellem Wissen und genetischen Ressourcen sowie zu nationalen Erfahrungen mit Rechtsschutzinstrumenten für Ausdrucksformen von Folklore. ${ }^{642}$ Diese Fragebögen richteten sich an die Mitgliedstaaten und die anderen Mitglieder des IGC: Internationale Organisationen, Vertreter von indigenen und lokalen Gemeinschaften sowie an betroffene Behörden, Forschungseinrichtungen und Experten im Bereich des geistigen Eigentums.

\section{Die Draft Provisions (Entwicklungen bis zur 10. Sitzung)}

In der 6. Sitzung des IGC im März 2004 wurde beschlossen, dass das Sekretariat auf Grundlage dieser gesammelten Informationen einen Überblick zu den allgemeinen Grundsätzen sowie den politischen und rechtlichen Spielräumen für die Themenbereiche TK und TCEs erarbeiten solle. ${ }^{643}$ Diese Dokumente wurden

gen: Der Fall der WIPO“, in: Regina Bendix, Kilian Bizer, und Stefan Groth (Hrsg.), Die Konstituierung von Cultural Property: Forscbungsperspektiven, 2010, S. 197-220.

${ }^{641}$ Diese Beschlüsse wurden bezugnehmend auf das vom Sekretariat vorbereitete Grundlagendokument getroffen. WIPO IGC, „Matters Concerning Intellectual Property and Genetic Resources, Traditional Knowledge and Folklore - An Overview", WIPO/GRTKF/IC/1/3, 16. März 2001; WIPO IGC, „First Session, April 30 to May 3, 2001, Report“, WIPO/GRTKF/IC/1/13, 23. Mai 2001, Abs. 128, 155, 175.

642 WIPO, „Key Issues, Gap Analysis and Consultations“, zugegriffen 14. Januar 2017, http://www.wipo.int/tk/en/igc/consultations.html.

${ }^{643}$ WIPO IGC, „Sixth Session, March 15 to 19, 2004, Report“, WIPO/GRTKF/IC/6/14, 14. April 2004, Abs. 66, 109; Dabei wurde auf die zuvor vom Sekretariat entwickelten Dokumente zu rechtlichen und politischen Möglichkeiten verwiesen: WIPO IGC, „Traditional Cultural Expressions/Expressions of Folklore Legal and Policy Options“, WIPO/GRTKF/IC/6/3, 
auf der folgenden Sitzung im November 2004 vorgestellt ${ }^{644}$ und in drei Phasen der Kommentierung vor der 8., der 10. sowie der 16. Sitzung von Seiten der Mitgliedstaaten und den anderen Mitgliedern des IGC entsprechend weiterentwickelt. ${ }^{645}$ Die Entwürfe veränderten sich so von einem ersten Overview of Policy Objectives and Core Principles in der 7. Sitzung durch die erste Kommentierungsphase in die Revised Objectives and Principles der 8. Sitzung. Die Diskussionen während der 8. und 9. sowie die schriftlichen Beiträge der zweiten Kommentierungsphase in Vorbereitung zur 10. Sitzungen wurden gesammelt, jedoch nicht in die entsprechenden Dokumente eingearbeitet. ${ }^{646}$

\section{Die 10 Schlüsselfragen und die Gap Analysis}

Während der 10. Sitzung erarbeitete das IGC zehn Schlüsselfragen (Key Issues) zum Schutz von TK/TCEs, die nach einer schriftlichen Kommentierungsphase eingehend während der 11. Sitzung im Juli 2007 diskutiert und schließlich zusammenfassend vom Sekretariat aufgearbeitet wurden. ${ }^{647}$ Während der 12. Sitzung im Februar 2008 wurde das Sekretariat beauftragt, auf Grundlage der bisherigen Erkenntnisse bestehende Regelungslücken des Schutzes von TK/TCEs

1. Dezember 2003; WIPO IGC, „Traditional Knowledge: Policy and Legal Options“, WIPO/GRTKF/IC/6/4, 12. Dezember 2003.

${ }^{644}$ WIPO IGC, „The Protection of Traditional Cultural Expressions/Expressions of Folklore:

Overview of Policy Objectives and Core Principles“, WIPO/GRTKF/IC/7/3, 20. August 2004; WIPO IGC, „Protection of Traditional Knowledge: Overview of Policy Objectives and Core Principles“, WIPO/GRTKF/IC/7/5, 20. August 2004.

645 WIPO, „Draft Provisions/Articles for the Protection of Traditional Knowledge and Traditional Cultural Expressions, and IP \& Genetic Resources“, zugegriffen 14. Januar 2017, http://www.wipo.int/tk/en/igc/draft_provisions.html.

${ }^{646}$ Obwohl in den Dokumenten keine inhaltlichen Änderungen vorgenommen wurden, wurden sie in der 10. Sitzung als Draft Objectives and Principles tituliert. In den folgenden Sitzung kehrte man aber zur Bezeichnung Revised Objectives and Prinicples zurück. WIPO IGC, „The Protection of Traditional Cultural Expressions/Expressions of Folklore: Draft Objectives and Principles“, WIPO/GRTKF/IC/10/4, 2. Oktober 2006, Abs. 7-9; WIPO IGC, „The Protection of Traditional Knowledge: Draft Objectives and Principles“, WIPO/GRTKF/IC/10/5, 2. Oktober 2006, Abs. 7-9.

${ }^{647}$ WIPO IGC, „Tenth Session, November 30 to December 8, 2006, Report“", WIPO/GRTKF/IC/10/7 Prov.2, 25. April 2007, Abs. 254; WIPO IGC, „Eleventh Session, July 3 to July 12, 2007, Report", WIPO/GRTKF/IC/11/15, 20. März 2008, Abs. 70-496; WIPO IGC, „The Protection of Traditional Cultural Expression/Expression of Folklore: Factual Extraction“, WIPO/GRTKF/IC/12/4(B), 31. Januar 2008; WIPO IGC, „The Protection of Traditional Knowledge: Factual Extraction“, WIPO/GRTKF/IC/12/5(B), 18. Februar 2008; WIPO IGC, „Protection of Traditional Cultural Expressions and Traditional Knowledge - Key Issues“, zugegriffen 14. Januar 2017, http://www.wipo.int/tk/en/igc/issues.html. 
zu analysieren. ${ }^{648}$ Ein erster Entwurf dieser Gap Analysis wurde durch Kommentare ergänzt und schließlich zur 13. Sitzung vorgestellt. ${ }^{649}$

\section{Die Draft Privisions (Weiterentwicklung ab der 15. Sitzung)}

Auf der 15. Sitzung wurde beschlossen, die Revised Objectives and Principles der 9. Sitzung wieder aufzunehmen. ${ }^{650}$ Unter Berücksichtigung der Kommentare der 15. Sitzung und weiterer schriftlicher Beiträge zu einer Vorabversion im Zuge der dritten Kommentierungsphase überarbeitete dann das Sekretariat die Revised Objectives and Principles. ${ }^{651}$ Für den Themenbereich TCEs wurden während der 16. Sitzung weitere Editierungsvorschläge und Kommentare eingebracht sowie schriftliche Beiträge im Anschluss der Sitzung gesammelt. Zum Thema TK wurde entsprechend auf der 16. und 17. Sitzung verfahren. Diese Dokumente bildeten die Grundlage für die folgenden Expertentreffen der Intersessional Workinggroups. ${ }^{652}$

\section{Die Intersessional Workinggroups}

Bereits während der 12. Sitzung wurde von der afrikanischen Gruppe der Vorschlag eingebracht, dass zwischen den Sitzungen Expertentreffen die rechtlichen Detailfragen klären sollten. ${ }^{653}$ Diese Intersessional Workinggroups wurden nach einem entsprechenden Beschluss der 13. Sitzung im Zuge der Mandatsverlängerung von der WIPO Generalversammlung eingerichtet. ${ }^{654}$ So leisteten die Ar-

${ }^{648}$ WIPO IGC, „,Twelfth Session, February 25 to 29, 2008, Report“, WIPO/GRTKF/IC/12/9, 21. November 2008, Abs. 257.

${ }^{649}$ WIPO IGC, „The Protection of Traditional Cultural Expressions: Draft Gap Analysis“, WIPO/GRTKF/IC/13/4(B) REV., 11. Oktober 2008; WIPO IGC, „The Protection of Traditional Knowledge: Draft Gap Analysis: Revision“, WIPO/GRTKF/IC/13/5(B) REV., 11. Oktober 2008; Eine Zusammenstellung der eingereichten schriftlichen Kommentare findet sich auf der Website des IGC: WIPO IGC, „Protection of Traditional Cultural Expressions and Traditional Knowledge - Gap Analyses“, zugegriffen 14. Januar 2017, http://www.wipo.int/tk/en/igc/gap-analyses.html.

${ }^{650}$ WIPO IGC, „Fifteenth Session, December 7 to 11, 2009, Report“, WIPO/GRTKF/IC/15/7, 14. Mai 2010, Abs. 124, 137.

${ }^{651}$ WIPO IGC, „The Protection of Traditional Cultural Expressions/ Expressions of Folklore: Revised Objectives and Principles“, WIPO/GRTKF/IC/16/4, 22. März 2010; WIPO IGC, „The Protection of Traditional Knowledge: Revised Objectives and Principles“, WIPO/GRTKF/IC/16/5, 22. März 2010.

${ }^{652}$ WIPO IGC, „The Protection of Traditional Cultural Expressions/Expressions of Folklore: Revised Objectives and Principles“, WIPO/GRTKF/IC/17/4, 21. September 2010, Abs. 2; WIPO IGC, „The Protection of Traditional Knowledge: Revised Objectives and Principles“, WIPO/GRTKF/IC/18/5, 10. Januar 2011, Abs. 1.

${ }_{653}$ WIPO IGC, ,Twelfth Session, February 25 to 29, 2008, Report“, WIPO/GRTKF/IC/12/9, Abs. 175, 257 (iv).

${ }^{654}$ WIPO Generalversammlung, ,, Thirty-Eighth (19th Ordinary) Session, Geneva, September 22 to October 1, 2009, Report“, WO/GA/38/20, 1. Oktober 2009, Abs. 217 (b). 
beitsgruppen zwischen der 16. und 18. Sitzung, mit je einer Sitzung zu den drei Themenbereichen GR, TK und TCEs, juristische und technische Beratung.

\section{Das Glossar}

Des Weiteren wurde während der 16. Sitzung das Sekretariat beauftragt, ein Glossar zu Begrifflichkeiten in Bezug zu geistigem Eigentum und genetische Ressourcen zu erstellen. ${ }^{65}$ Entsprechende Glossare wurden auf der 17. Sitzung auch für die Themen traditionelles Wissen und traditionelle kulturelle Ausdrucksformen beim Sekretariat in Auftrag gegeben. ${ }^{656}$ Auf der 19. Sitzung wurde das Sekretariat schließlich beauftragt, aus den drei Glossaren ein umfassendes Dokument zu erstellen, welches die Begrifflichkeiten und Definitionen der drei Themenbereiche erklärt. ${ }^{657}$

\section{Die Draft Provisions (Überarbeitung durch die Intersessional Workinggroups)}

Im Zuge der Intersessional Workinggroups zwischen der 16. und 18. Sitzung wurden aus den Revised Objectives and Principles von den Experten Vorschläge von draft articles für die Themenbereiche TK und TCE erarbeitet. Für die Verhandlungen zu GRs veränderte sich der Status der Dokumente von der Revised List of Options im Zuge der Intersessional Workinggroup zu Draft Objectives and Principles in Verbindung mit einem Dokument zu Options for Future Work on Intellectual Property and Genetic Resources. Diese Dokumente wurden auf der 18. und 19. Sitzung weitergehend beraten. Schließlich wurde für das folgende Biennium 2012/13 ein intensiver Zeitplan mit jeweilig individuellen Sitzungen zu den drei Themenbereichen beschlossen. 658

\footnotetext{
655 WIPO IGC, „Sixteenth Session, May 3 to May 7, 2010, Draft Report“, WIPO/GRTKF/IC/16/8 Prov.2, 30. September 2010, Abs. 252.

656 WIPO IGC, „Seventeenth Session, December 6 to 10, 2010, Report“, WIPO/GRTKF/IC/17/12, 6. Juni 2011, Abs. 338, 349.

${ }_{657}$ WIPO IGC, „Nineteenth Session, July 18 to 22, 2011, Report“, WIPO/GRTKF/IC/19/12, 23. Februar 2012, Abs. 174. 658 Ebd., Abs. 563.
} 
IV. Tabellarische Darstellung der Verhandlungen des WIPO IGC

\begin{tabular}{|c|c|c|}
\hline IGC & Daten & Inhalt \\
\hline & $25.9-3.10 .2000$ & $\begin{array}{l}\text { WIPO GA gründet IGC: WIPO, WO/GA/25/10, } \\
27-71 \text {, Item } 15 \text {, WO/GA } / 26 / 6,13,16-18\end{array}$ \\
\hline 1 & Dez 2001 & $\begin{array}{l}\text { Questionaire on national experience with the } \\
\text { legal protection of expressions of folklore } \\
\text { (WIPO/GRTKF/IC/2/7) }\end{array}$ \\
\hline 6 & Mär 2004 & Die Draft Provisions werden in Auftrag gegeben \\
\hline 7 & Nov 2004 & $\begin{array}{l}\text { Review der Draft Provisions } \\
\text { (WIPO/GRTKF/IC/7/3 (TCEs) and } \\
\text { WIPO/GRTKF/IC/7/5 (TK)) }\end{array}$ \\
\hline $7-8$ & $\begin{array}{l}\text { Nov } 04-\text { Feb } \\
05\end{array}$ & First Commenting Process on Draft Provisions \\
\hline $9-10$ & Apr $06-$ Dez 06 & Second Commenting Process on Draft Provisions \\
\hline 10 & Dez $06-$ Jul 07 & Identifizierung von List of Key Issues \\
\hline $10-11$ & & $\begin{array}{l}\text { Commentary Process for these } 10 \text { Key Issues } \\
\text { ( } 25 \text { Antworten); Review by the committee } \\
\text { (WIPO/GRTKF/IC/12/4(b) and } \\
\text { WIPO/GRTKF/IC/12/5(b)) }\end{array}$ \\
\hline 13 & Okt 2008 & $\begin{array}{l}\text { Two (TCEs/EoF \& TK) Gap Analysis } \\
\text { (WIPO/GRTKF/IC/13/4(b) Rev. and } \\
\text { WIPO/GRTKF/IC/13/5(b) Rev.) auf Grundlage } \\
\text { von Consultation Drafts mit darauf folgendem } \\
\text { Intersessional Review Process }\end{array}$ \\
\hline $15-16$ & Dez $09-$ Feb 10 & $\begin{array}{l}\text { Third Commenting Process on Draft Provisions } \\
\text { (Intersessional Written Commenting Process) }\end{array}$ \\
\hline \multirow[t]{2}{*}{16} & Mär 2010 & Einrichtung der Intersessional Workinggroups \\
\hline & Jul 10 - Mär 11 & $\begin{array}{l}\text { Intersessional Workiggroups zu TCE/EoF, TK und } \\
\text { GR }\end{array}$ \\
\hline $17-19$ & Dez $10-$ Jul 11 & $\begin{array}{l}\text { Diskussion der Ergebnisse der Intersessional Work- } \\
\text { inggroups }\end{array}$ \\
\hline $20-22$ & Feb - Jul 2012 & 3 Themensitzungen innerhalb von 6 Monaten \\
\hline
\end{tabular}




\section{Positionen im IGC}

In den Äußerungen während der Verhandlungen des IGC, den Sitzungen der WIPO Generalversammlung zu diesem Themenbereich sowie den verschiedenen schriftlichen Kommentaren zu den einzelnen Aktivitäten im Rahmen der Verhandlungen zeichnen sich die Verhandlungspositionen einzelner Mitgliedstaaten ab. Das IGC strukturiert sich dabei, wie jede Sonderorganisation der Vereinten Nationen, in die informellen Zusammenschlüsse der Staatengruppen, die sich auf Grundlage historischer, wirtschaftlicher und sozialer Gemeinsamkeiten geographisch einordnen lassen. Vor dem Hintergrund dieser Gruppenstrukturen entscheiden sich im UN-System insbesondere Wahlen zu bestimmten Positionen sowie gemeinsame Verhandlungspositionen. ${ }^{659}$ In der WIPO dienen die Sprecher der Gruppen auch der besseren Kommunikation zwischen dem Sekretariat und dem Plenum. ${ }^{660}$

Die Gruppenbildung in der WIPO gestaltete sich schwieriger als in anderen internationalen Organisationen, da sich gemeinsame Interessen im Themenbereich geistiges Eigentum insbesondere im Kontext von Entwicklung nicht durch bloße geographische Nähe ausmachen lassen. Vielmehr ergibt sich durch überlagernde Mitgliedschaften in der OSZE und in verschiedenen Freihandelsabkommen mit TRIPS + Verpflichtungen ein komplexes Feld verschiedener Interessen, die eine Koordinierung unter den Entwicklungsländern in der WIPO schwierig machen. ${ }^{661}$ Dennoch lässt sich das $I G C$ vereinfacht in folgende Gruppen strukturieren: die Gruppe der lateinamerikanischen und karibischen Staaten (Group of Latin American and Caribbean States, GRULAC), die afrikanische Gruppe, die Gruppe B der Industriestaaten, die Asien und Parifik. Gruppe, die Gruppe der zentral europäischen und baltischen Staaten, die Gruppe der osteuropäischen und zentralasiatischen Staaten sowie China. ${ }^{662}$ Außerdem hat sich im Verlauf der Verhandlungen eine überregionale Gruppe von like-

${ }^{659}$ Justin S. Gruenberg, „An Analysis of United Nations Security Council Resolutions: Are All Countries Treated Equally?", Case Western Reserve Journal of International Law, Vol. 41, Nr. 2-3 (2009), S. 477ff.; Ahmed Abdel Latif, „Developing Country Coordination in International Intellectual Property Standard-setting“, Trade-related Agenda, Development and Equity (T.R.A.D.E.), Working Papers 24, South Centre, 2005, S. 32.

${ }^{660}$ Latif, „Developing Country Coordination in International Intellectual Property Standard-setting“, S. 32 .

${ }^{661}$ Ebd., S. $31 \mathrm{ff}$.

${ }^{662}$ Ebd., S. 32; Centre for International Environmental Law (CIEL), A Citizen's Guide to WIPO, 2007, S. 32; Außerdem nehmen Palästina als Beobachter sowie der Heilige Stuhl an den Verhandlungen teil. Letzterer machte seine Position in einem eigenen Dokument zu Beginn der Verhandlungen deutlich: WIPO IGC, „Document of the Holy See on Intellectual Property and Genetic Resources, Traditional Knowledge and Folklore“, WIPO/GRTKF/IC/1/7, 26. April 2001. 
minded states gefunden, die ähnlich zur gleichnamigen Gruppe in der CBD gemeinsame Positionen vertritt. 663

\section{Die Gruppe lateinamerikanischer und karibischer Staaten}

Die GRULAC brachte bereits zur 26. Sitzung der WIPO Generalversammlung ein Positionspapier ein, in dem sie ihren progressiven Ansatz deutlich machte und lieferte somit die Diskussionsgrundlage für die erste Sitzung des IGC. GRULAC vertritt darin, dass technisches Wissen nicht nur in Form der bisher durch Instrumente geistigen Eigentums geschützten Verfahren entstehe. Vielmehr werden solches Wissen, Innovationen und Praktiken auch von indigenen und lokalen Gemeinschaften geschaffen. ${ }^{664} \mathrm{Im}$ Bereich von Folklore beispielsweise sei der Schutz vor Reproduktion von kulturellen Ausdrucksformen durch das Urheberrecht schwer oder gar unmöglich zu realisieren. Dies liegt unter anderem daran, dass der Urheber im Sinne des Urheberrechts nicht zu bestimmen sei bzw. nur konkrete Ausdrucksformen, nicht aber bestimme Konzepte, Ideen oder Stile geschützt seien. ${ }^{665}$ Infolge dessen, so GRULAC, würden Interessen der Gemeinschaften, von denen diese Ausdrucksformen stammen, durch unerlaubte Nutzungen in Form von Nachahmung oder Vervielfältigung und kommerziellen Missbrauch verletzt. Hingegen stünden verschiedene Mechanismen wie zum Beispiel eine domaine public payant, Regeln des unlauteren Wettbewerbs oder Regeln der ungerechtfertigten Bereicherung zur Verfügung, um für einen Interessenausgleich zu sorgen. ${ }^{666}$

In ihrem Positionspapier macht GRULAC auch ihr Verständnis von „,traditionellem "Wissen im weiteren Sinne" deutlich, welches neben TK im engeren Sinne auch Innovationen, Marken, Folklore, Artefakte sowie bestimmte Designs umfassen soll und zeigt konkrete Vorschläge auf, wie Defizite von bestehenden Schutzinstrumenten für dieses Wissen ausgeglichen werden könnten. ${ }^{667}$

Die GRULAC regt schließlich an, das bestehende System geistigen Eigentums zu überarbeiten, um damit nicht nur den Interessen der Mitgliedstaaten und der Öffentlichkeit im Ganzen nachzukommen, sondern explizit auch den Interessen von indigenen und lokalen Gemeinschaften gerecht zu werden. ${ }^{668}$ Die Forderung

\footnotetext{
${ }^{663}$ Beiden Gruppen der like-minded states gehören Kolumbien, Indien, Indonesien, Malaysia, Peru und Südafrika an.

${ }^{664}$ WIPO IGC, „Traditional Knowledge and the Need to Give it Adequate Intellectual Property Protection. WIPO Committee on the Relationship between Intellectual Property, Genetic Resources and Traditional Knowledge (documents submitted by the Group of Countries of Latin America and the Caribbean (GRULAC)“, WIPO/GRTKF/IC/1/5, 16. März 2001, Annex I, S. 1.

${ }^{665} \mathrm{Ebd}$.

${ }^{666}$ Ebd., Annex I, S. 2.

${ }^{667}$ Ebd., Annex I, S. 6f.

${ }^{668}$ Ebd., Annex I, S. 9; Annex II, S. 5.
} 
nach einem kollektivrechtlichen Ansatz wird von Seiten $\operatorname{der}$ GRULAC als eine Kernforderung in die Verhandlungen eingebracht.

\section{Die afrikanische Gruppe}

Auch die African Group machte mit einem Positionspapier beim ersten Treffen des $I G C$ ihre Vorstellungen deutlich. ${ }^{609}$ Darin ist, anders als in dem Positionspaper der GRULAC, welches den problematischen Umgang mit TK und TCEs als Ausgangspunkt wählt, das gegenwärtige System geistigen Eigentums zentraler Bezugspunkt. Es werden die konzeptionellen Probleme geistigen Eigentums, TKs und der Ausdrucksformen von Folklore dargestellt, verglichen und schließlich daraus der als notwendig betrachtete Änderungsbedarf und die damit einhergehenden Erwartungen an das $I G C$ formuliert.

Explizit wird von der African Group eine auf unterschiedlichen Trägern beruhende Differenzierung zwischen, indigenous knowledge' und ,traditional knowledge' deutlich gemacht. ${ }^{670}$,Indigenes Wissen ${ }^{6}$ kann verstanden werden als von einer Gruppe getragen, die sich selbst als indigen an einem Ort versteht. Beruhend auf einer Kombination von kultureller Unterschiedlichkeit und einer im Verhältnis zu einer neueren Bevölkerung vorausgehenden territorialen Verbindung sollen ,indigene Gruppen' gegenüber der dominierenden Kultur unterschieden werden können. ${ }^{671} T K$ hingegen sei solches Wissen, welches von Mitgliedern einer bestimmten Kultur getragen und in einer der Kultur eigenen Art der Aneignung oder Forschung entwickelt wurde und wird und dabei in Bezug zu Kultur oder Umwelt der Gruppe stehe. ${ }^{672}$ Indigenes Wissen kann somit TK sein, während TK nicht notwendigerweise auch indigenes Wissen sein muss. Der Unterschied zwischen TK und indigenous knowledge' liegt somit darin, dass letzteres von einer Gruppe getragen wird, die ihre Geschichte an einem bestimmten Ort auf vorkoloniale Zeiten zurückführen kann. Der allgemeine Begriff TK hingegen umfasst die Gesamtheit all desjenigen Wissens, welches explizit oder implizit die sozio-ökonomische und ökologische Lebensführung ausmacht.

Dieses traditionelle Wissen, so die Position der African Group, stehe normalerweise in kollektivem Eigentum einer Gesellschaft, da es durch Beiträge vieler Mitglieder über Generationen hinweg bereichert und angepasst werde und sich somit konkret in einen sozio-ökologischen Kontext von wirtschaftlichen, kulturellen und religiösen Gegebenheiten einfüge. ${ }^{673}$ Das steigende Interesse an TK und me-

\footnotetext{
${ }^{669}$ WIPO IGC, „Proposal Presented by the African Group to the First Meeting of the Intergovernmental Committee on Intellectual Property and Genetic Resources, Traditional Knowledge and Folklore (submitted by the African Group)“, WIPO/GRTKF/IC/1/10, 1. Mai 2001.

${ }^{670}$ Ebd., Annex, S. 1.

671 Ebd.

672 Ebd.

${ }^{673}$ Ebd., Annex, S. 2.
} 
dizinalem Wissen der letzten Jahre hat deren Wichtigkeit für neue Pharmaprodukte steigen lassen und somit einen erheblichen Beitrag in diesem Sektor geleistet. Dennoch mangelt es oft an der Anerkennung und dem Schutz der indigenen und lokalen Gemeinschaften von denen dieses Wissen stamme sowie an einer angemessenen Beteiligung an den Gewinnen, die aus der kommerziellen Nutzung dieser neuen Produkte erzielt würden. Beispielhaft wird im Positionspapier der African Group ausgeführt, wie die Paris Convention, der CBD, das TRIPs Abkommen, die International Convention for the Protection of New Varieties of Plants (UPOV) sowie das International Undertaking on Plant Genetic Resources der FAO jeweils Interpretationsmöglichkeiten bieten, die einen kollektivrechtlichen Schutz für indigene und lokale Völker zulassen würden. Schließlich wird das Model Law ${ }^{674}$ der Organization of African Unity $(O A U)$ zu Rechten lokaler Gemeinschaften, Landwirten und Züchtern und zur Regelung des Zugangs zu biologischen Ressourcen vorgestellt, unter dem Gemeinschaften das Recht zugesprochen wird, den Zugang zu ihren Ressourcen und ihrem Wissen untersagen zu können, sollte ein solcher die Integrität ihres Natur- oder Kulturerbes schädigen. Des Weiteren wird der Staat verpflichtet, 50 Prozent der Gewinne, die durch die Nutzung dieser Ressourcen oder das Wissen entstehen, an die Gemeinschaften zurückzuführen. ${ }^{675}$

Im Bereich des Schutzes von Ausdrucksformen von Folklore wird mit Bezügen zu verschiedenen nationalen Regelungen deutlich gemacht, wie über das Urheberrecht jeweils staatliche Instanzen die Nutzung von Folklore gegen Entgelt gestatten können. So liegen beispielsweise die Rechte an Folklore in Ghana beim Staat, bei dem für bestimmte Nutzungen gegen Entgelt eine Lizenz erworben werden kann. In Nigeria wurde ein Rat für Urheberrecht eingerichtet, der den Schutz von Folklore überwacht. In Tunesien bedarf die kommerzielle Verwertung von Ausdrucksformen von Folklore der ausdrücklichen Zustimmung durch das Kultusministerium. ${ }^{676}$

In der Position der African Group wird somit ebenfalls ein kollektivrechtlicher Ansatz deutlich, der lokale und indigene Gemeinschaften als Berechtigte an einem access and benefit sharing an traditionellem Wissen und traditionellen kulturellen Ausdrucksformen beteiligen möchte. Gleichzeitig wird mehrfach der Vorschlag geäuBert, den Staat als ausführenden Akteur, als competent authority, zur Ausübung entsprechender Rechte hinzuzuziehen.

\footnotetext{
${ }^{674}$ Organisation of the African Unity, „Model Legislation for the Recognition and Protection of the Rights of Local Communities, Farmers and Breeders, and for the Regulation of Access to Biological Resources", gebilligt im Rahmen der 74. Sitzung des Ministerrates der OAU vom 5.-8. Juli 2001, CM/Dec.44 (LXXIV).

675 WIPO IGC, „Proposal Presented by the African Group“, WIPO/GRTKF/IC/1/10, Annex, S. 5. 676 Ebd.
} 


\section{Die Gruppe B}

Im Gegensatz zu den progressiven Positionen der GRULAC und der afrikanischen Gruppe zeigt die Gruppe B eine zurückhaltende und teilweise verzögernde Teilnahme an den Verhandlungen. So machte die EU in ihrem Positionspapier zum Schutz von Folklore für die Verhandlungsperspektive folgendes deutlich:

When looking ahead, we should be guided by:

- the conclusions of previous WIPO discussions;

- the replies to the Questionnaire;

- the need to draw a clear line between intellectual property

protection and the public domain;

- the need not to dilute intellectual property protection. ${ }^{677}$

Ziel der Beteiligung der Gruppe B ist es demnach, das bestehende System geistigen Eigentums gegen eine Aufweichung zu verteidigen und gleichzeitig neuen Schutzrechten für immaterielle Werte entgegenzutreten, die bislang in der public domain liegen. Die Mitglieder der Gruppe B beteiligen sich zwar aktiv an den Verhandlungen zu TK und TCEs und partizipieren an den Aktivitäten des IGC wie den Fragebögen und weiteren Initiativen zur Sammlung von Informationen. Sie brachten aber im Vergleich zur Gruppe GRULAC und der afrikanischen Gruppe weniger konstruktive Vorschläge und Arbeitsdokumente ein. Beispielhaft für die Perspektive und den diplomatischen Beitrag der Staaten der Gruppe B für die Themenbereiche TK und TCE ist folgendes opening statement der EU in der 18. Sitzung im Jahr 2011:

It stood committed to participating in a constructive manner at the meeting, and would intervene on specific issues of the draft articles at the appropriate moment. It also looked forward to commenting on the objectives and principles of the text. However, it recalled that it was strongly committed to a nonbinding approach. It was confident that the meeting would pave the way for reaching consensus among the diverging positions. ${ }^{678}$

Die wenig konstruktive Beteiligung der Vertreter der Gruppe B zeigt deutlich die ablehnende Haltung der westlichen Staaten zur Schaffung neuer Rechte geistigen Eigentums an traditionellen kulturellen Ausdrucksformen und traditionellem Wissen. Die formelle Beteiligung im diplomatischen Austausch der Standpunkte zu den verhandelten Inhalten lässt in den Nachfragen und den weiteren

\footnotetext{
677 WIPO IGC, „Expressions of Folklore, Document submitted by the European Community and its Member States", WIPO/GRTKF/IC/3/11, 16. Mai 2002, Annex, S. 3.

678 WIPO IGC, „Eighteenth Session, May 9 to 13, 2011, Report“, WIPO/GRTKF/IC/18/11, 29. Juli 2011, Abs. 40.
} 
Initiativen zu Begriffsklärungen und Informationssammlung das Ziel einer Verhandlungsverzögerung erkennen.

Intensiver gestalten sich die Aktivitäten der Gruppe B zum Themenkomplex genetische Ressourcen. Dies zeigt sich beispielsweise in der gemeinsamen Vorlage Australiens, Kanadas, Japans, Norwegens, Neuseelands und der USA in der 16. Sitzung. ${ }^{679}$ Darin wurden grundlegende Prinzipien entsprechend den Schutzzielen formuliert, welche grundlegende Beachtung im weiteren Verhandlungsverlauf gefunden haben. ${ }^{680}$

\section{Die Asien und Pazifik Gruppe und China}

Zur zweiten Sitzung des IGC reichten die Asian Group und China ein gemeinsames Positionspapier ein, aus dem ihre Erwartungen und Perspektiven zum IGC deutlich werden. ${ }^{681}$ Darin kommt zum Ausdruck, dass sie die WIPO für das am besten geeignete Forum zur Entwicklung einer international anerkannten Lösung zu Fragen des geistigen Eigentums im Kontext von genetischen Ressourcen, traditionellem Wissen und Folklore halten. Allerdings regen sie für jeden der drei Themenblöcke an, dass auf nationaler Ebene entsprechende Konfliktfälle gelöst werden sollten sowie die Geltung von anderen internationalen Instrumenten wie beispielsweise der $C B D$ nicht zu beeinträchtigen seien. Schließlich sollen im Rahmen des IGC von Seiten der WIPO zunächst umfassende Informationen zusammengetragen und ausgewertet werden, um im Rahmen der bestehenden Instrumente einen ausreichenden Schutz zu gewährleisten.

Eine detaillierte Darstellung der Probleme und des eigenen Verständnisses der diskutierten Themen finden ebenso wenig statt wie explizite Vorschläge zu konkreten Regelungen oder gangbaren Kompromissen.

\section{Balkan und osteuropäische Staaten sowie zentraleuropäische und baltische Staaten}

Die Balkan- und osteuropäischen Staaten treten ebenso wie die zentraleuropäischen und baltischen Staaten während der Verhandlungen kaum in Erscheinung. Beide Gruppen zeigen keine progressive Unterstützung zu den verhandelten Themen und reihen sich so in die Verhandlungsperspektive der Gruppe B und der asiatischen Staaten ein. Die gemeinsame Position zeigt sich beispielsweise in dem während der 15. Sitzung eingereichten gemeinsamen Papier zur Arbeitsweise der Intersessional Workinggroups von Gruppe B und der zentraleuropäischen und baltischen

\footnotetext{
${ }^{679}$ WIPO IGC, „Submission by Australia, Canada, New Zealand, Norway and the United States of America“, WIPO/GRTKF/IC/16/7, 6. Mai 2010.

680 WIPO IGC, „Draft Objectives and Principles Relating to Intellectual Property and Genetic Resources prepared at IWG 3“, WIPO/GRTKF/IC/18/9, 17. März 2011, Abs. 2.

681 WIPO IGC, „Position Paper of the Asian Group and China (submitted by the Asian Group and China)“, WIPO/GRTKF/IC/2/10, 3. Dezember 2001.
} 
Staaten. ${ }^{682}$ Abgesehen von solchen Kooperationen halten sich die beiden Staatengruppen grundsätzlich zurück.

\section{Like-minded countries}

Im Zuge der Verhandlungen formierte sich eine überregionale Gruppierung der like-mindend countries, die mit konstruktiven Vorschlägen, ähnlich den Beiträgen der GRULAC und der afrikanischen Gruppe, versucht, den Verhandlungsverlauf zu beschleunigen. ${ }^{683}$ Diese Gruppe setzt sich dabei aus jeweils wenigen Staaten der GRULAC, der afrikanischen Gruppe und der asiatischen Gruppe zusammen. ${ }^{684}$ Aus ihren Vorlagen zu den drei Themenbereichen ${ }^{685}$ und ihren allgemeinen Vorschlägen zu den Verhandlungen spricht ein progressiver und konstruktiver Ansatz. In ihren Bali Empfehlungen fordern sie das IGC dazu auf, der WIPO Generalversammlung zu empfehlen:

1. to convene a Diplomatic Conference in 2013;

2. to renew the mandate of the IGC to continue its work and undertake text-based negotiations with the objective of reaching agreement on a text/s of an international legal instrument (or instruments) which would be submitted to the Diplomatic Conference in 2013;

3. to convene adequate number of Special Sessions of the IGC during the biennium, in addition to the four regular sessions of the IGC, to facilitate timely finalization of the text/s. To this end, a detailed work program, including a schedule of special

\footnotetext{
682 WIPO IGC, „Proposal of Group B and Group of Central European and Baltic States for Intersessional Working Groups for the Intergovernmental Committee“, WIPO/GRTKF/IC/15/6, 11. Dezember 2009.

${ }^{683}$ Zum ersten mal trat die Gruppe der like-minded countries auf der 15. Sitzung in Erscheinung. WIPO IGC, „Fifteenth Session, December 7 to 11, 2009, Report“, WIPO/GRTKF/IC/15/7, 14. Mai 2010, Abs. 40.

${ }^{684}$ Diese Gruppe besteht aus Algeria, Angola, Bangladesh, Colombia, Egypt, India, Indonesia, Malaysia, Myanmar, Namibia, Pakistan, Peru, South Africa, Tanzania, Thailand, and Zimbabwe. Siehe WIPO IGC, „Recommendations of the Second Session of Like Minded Countries Meeting on the Protection of Genetic Resources, Traditional Knowledge and Folklore“, WIPO/GRTKF/IC/19/8, 8. Juli 2011.

${ }^{685}$ WIPO IGC, „Like-Minded Countries Contribution to the Draft Articles on the Protection of Traditional Cultural Expressions“, WIPO/GRTKF/IC/19/9, 18. Juli 2011; WIPO IGC, „Like-Minded Countries Contribution to the Draft Articles on the Protection of Traditional Knowledge“, WIPO/GRTKF/IC/19/10, 18. Juli 2011; WIPO IGC, „Like-Minded Countries Contribution to the Objectives and Principles on the Protection of Genetic Resources and Preliminary Draft Articles on the Protection of Genetic Resources“, WIPO/GRTKF/IC/19/11, 18. Juli 2011.
} 
sessions of the IGC, shall be adopted by the General Assembly. ${ }^{686}$

Die like-minded countries treten für eine Intensivierung der Arbeit des IGCs mit zusätzlichen Sitzungen und dem Ziel einer Diplomatic Conference zur Verabschiedung eines rechtsverbindlichen Instruments innerhalb von zwei Jahren ein. Dabei zeigt gerade die Forderung nach einem Abschluss der Verhandlungen bis 2013, dass die like-minded countries den Druck der Verhandlungen erhöhen wollen und dabei auch ein Scheitern des IGC in Kauf nehmen. Ein Scheitern des IGC würde ihre Verhandlungsposition im Gefüge weiterer internationaler Foren verwandter Themenbereiche in der CBD und der WTO stärken, da ein Verweis auf die laufenden Verhandlungen im IGC nicht mehr möglich wäre. ${ }^{687}$

\section{Positionen der Vertreter von Nichtregierungsorganisationen und indigener Völker}

Neben den WIPO Mitgliedstaaten sind über 200 NGOs zu den Verhandlungen im IGC zugelassen, von denen ein Großteil indigene Völker und lokale Gemeinschaften repräsentieren. ${ }^{688}$ In Anbetracht des Verhandlungsgegenstandes wird die Beteiligung indigener und lokaler Gemeinschaften als wichtig und notwendig empfunden, so dass neben regionalen workshops die Beteiligung indigener und lokaler Gruppen durch einen Fonds unterstützt wird, der ihnen die Reise nach Genf ermöglicht. ${ }^{689}$ Neben einem Rederecht während der Sitzungen und der Beteiligung in den Expertentreffen tragen die Vertreter indigener und lokaler Gruppen seit der 8. Sitzung in Form eines eigenen halbtägigen Panels zu Beginn jeder Sitzung zu den Verhandlungen bei. ${ }^{690}$ In ihren Beiträgen machen sie dabei ihre progressive Position zur Schaffung neuer Instrumente geistigen Eigentums zum Schutz von GR/TK/TCEs deutlich.

686 WIPO IGC, „Recommendations of the Second Session of Like Minded Countries Meeting on the Protection of Genetic Resources, Traditional Knowledge and Folklore“, WIPO/GRTKF/IC/19/8, 8. Juli 2011, Annex.

687 Groth, Negotiating Tradition - The Pragmatics of International Deliberations on Cultural Property, S. 48.

${ }^{688}$ Eine Liste aller akkreditierten Organisationen findet sich unter: WIPO IGC, „Participating in the IGC“, zugegriffen 14. Januar 2017, http://www.wipo.int/tk/en/igc/participation.html; Siehe auch: Groth, Negotiating Tradition - The Pragmatics of International Deliberations on Cultural Property, S. $50 \mathrm{f}$.

689 WIPO IGC, „Participation of Local and Indigenous Communities in the Work of the Committee“, WIPO/GRTKF/IC/4/12, 20. Oktober 2002, Abs. 3, 7, 11; Die Entscheidung zur Einrichtung des Fonds fiel in der 9. Sitzung: WIPO IGC, „Decisions of the Ninth Session of the Committee“, WIPO/GRTKF/IC/9/DECISIONS, 8. April 2006, Abs. 4f; WIPO Generalversammlung, „Establishment of the WIPO Voluntary Fund for Accredited Indigenous and Local Communities", Annex zu WO/GA/32/6, geändert durch WO/GA/39/11.

${ }^{690}$ WIPO IGC, „Decisions adopted by the Committee at it's Seventh Session, Geneva, November 1 to 5, 2004“, WIPO/GRTKF/IC/7/Decisions, 5. November 2004, Abs. 3 (iv). 
Die Beteiligung von Vertretern indigener und lokaler Gemeinschaften in multilateralen Verhandlungen im Rahmen internationaler Organisationen ist im Völkerrecht immer noch die Ausnahme. Hier hat jedoch die Thematik eine Beteiligung dieser Gruppen nahe gelegt. Die Art und Weise der Einbeziehung nichtstaatlicher Vertreter führte dennoch - nicht nur mangels Stimmrechte - zu Ernüchterung. So erklärten die indigenen und lokalen Gemeinschaften zur 20. Sitzung die Verweigerung der weiteren Teilnahme, da die Staatenvertreter ihren Vorstellungen einer angemessenen Beteiligung ihrer Gruppen noch nicht nachgekommen seien. ${ }^{691}$ In ihrer Presseerklärung machten die Vertreter indigener Völker dies folgendermaßen deutlich:

Distinguished delegates: we, the Indigenous Peoples, are the titleholders, proprietors and ancestral owners of traditional knowledge that is inalienable, nonforfeitable and inherent to the genetic resources that we have conserved and utilized in a sustainable manner within our territories. For this reason, we appeal to the States to acknowledge that the discussion on intellectual property rights and genetic resources should include Indigenous Peoples on equal terms with the States since the work will directly impact our lives, our lands, our territories and resources, and will reach to the very heart of our cultures, which are the inheritance of future generations.

Therefore, the Indigenous Peoples present at IGC 20 have reflected seriously on our role in this process and have decided, unanimously, to withdraw our active participation in the work developed by this Committee until the States change the rules of procedure to permit our full and equitable participation at all levels of the IGC and until the instruments recognize and are consistent with the existing international frameworks for the rights and interests of Indigenous Peoples within the scope of the IGC. ${ }^{692}$

Diese Aussage offenbart die Frustration über die mangelnde gleichberechtigte Beteiligung indigener und lokaler Gemeinschaften. Zudem wird eine intensive Auseinandersetzung mit den inhaltlichen Beiträgen indigener und lokaler Gruppen gefordert, die von den Staatenvertretern des IGC nicht nur formell zur Kenntnis genommen werden sollen. Es zeigt sich, dass die offiziell immer wieder betonte Teilnahme indigener und lokaler Gemeinschaften an den Verhand-

\footnotetext{
${ }^{691}$ Catherina Saez, „Indigenous Peoples Walk Out Of WIPO Committee On Genetic Resources“, Intellectual Property Watch, 22. Februar 2012.

${ }^{692}$ „IPs to withdraw from active participation in World Intellectual Property Organization (WIPO) Inter Governmental Committee“, Asia Indigenous Peoples Pact, 21. Februar 2012.
} 
lungen im $I G C$ in der Praxis nur geringfügig über einen Beobachterstatus hinausgeht.

\section{Zusammenfassung des Verlaufs der Verhandlungen und Positionen}

Die Verhandlungen im IGC zu neuen kollektiven Instrumenten geistigen Eigentums an GR/TK/TCEs zeigen die widerstreitenden Positionen zwischen dem globalen Süden und den westlichen Staaten. Die Staaten des globalen Südens bemühen sich progressiv um einen Abschluss der Verhandlungen, während die westlichen Staaten durch Anfragen zur weiteren Begriffsklärung und neuen Initiativen von Fragebögen und fact-finding missions den Beratungsprozess verlängern. Ob die Verhandlungen in Anbetracht dieser entgegengesetzten Verhandlungspositionen mit kleinsten Schritten minimaler Ergebnisse in naher Zukunft zu einem Kompromiss finden werden, bleibt abzuwarten. ${ }^{693}$ Allein der Verlauf der Verhandlungen sowie die verschiedenen Initiativen, die vom Sekretariat betrieben werden, bieten jedoch die Möglichkeit, die Thematik im öffentlichen Diskurs zu verbreiten und inhaltlich zu vertiefen. So ergibt sich durch die Beteiligung von Vertretern von Nichtregierungsorganisationen und indigenen Völkern ein Bewusstsein der Problematik, welches entsprechende Debatten auf nationaler und lokaler Ebene initiiert. Des Weiteren ist die Thematisierung des Zusammenhangs von geistigem Eigentum und GR/TK/TCEs Anstoß für weitere wissenschaftliche Untersuchungen und führt so zu alternativen Lösungsansätzen im Rahmen der bereits bestehenden Instrumente. ${ }^{694}$

\section{Subject Matter of Protection}

Die Verhandlungen des IGCs haben trotz der defensiven Beteiligung der westlichen Staaten in den letzten zehn Jahren erste Entwürfe von draft articles zum Schutz von TCEs und TK hervorgebracht. Auch wenn diese Entwürfe fortlaufend Gegenstand weiterer Verhandlungen sind und eine umfassende Zustimmung bisher noch nicht gegeben ist, lassen sich daraus die zugrunde liegenden Konzepte und Ideen neuer Rechte geistigen Eigentums erkennen. Eine Beschreibung der geschützten Werte TKs/TCEs findet sich in den Entwürfen jeweils in Artikel 1 der draft articles in der Beschreibung der Subject Matter of Protection.

\footnotetext{
${ }^{693}$ Lankau und Bizer, „Die verborgene Effektivität minimaler Resultate in internationalen Verhandlungen: Der Fall der WIPO“.

${ }^{694}$ Siehe dazu beispielsweise die Studie von Dagne: Teshager Worku Dagne, Intellectual Property, Traditional Knowledge and Biodiversity in the Global Economy: The Potential of Geographical Indications for Protecting Traditional Knowledge-Based Agricultural Products, 2012.
} 


\section{Traditional Cultural Expressions}

Die draft articles beschreiben traditionelle kulturelle Ausdrucksformen in Artikel 1 als jegliche gegenständlichen oder nichtgegenständlichen Formen, in denen sich traditionelle Kultur oder Wissen verkörpern, die von Generation zu Generation weitergegeben werden und nennt folgende Beispiele:

(a) phonetic or verbal expressions, such as stories, epics, legends, poetry, riddles and other narratives; words, [signs,] names, [and symbols];

(b) musical or sound expressions, such as songs, [rhythms,] and instrumental music, the sounds which are the expression of rituals;

(c) expressions by action, such as dances, plays, ceremonies, rituals, rituals in sacred places and peregrinations, [sports and [traditional]] games, puppet performances, and other performances, whether fixed or unfixed;

(d) tangible expressions, such as material expressions of art, [handicrafts,] [works of mas,] [architecture,] and tangible [spiritual forms], and sacred places. ${ }^{695}$

Das Verständnis von traditionellen kulturellen Ausdrucksformen bezieht sich somit auf gegenständliche oder nichtgegenständliche Formen von Lauten und Sprache, Musik, Bewegungen, Tänze, Schauspiel, Rituale, Kunst und auch Orte. Absatz 2 macht deutlich, dass sich der rechtliche Schutz jedoch nur auf folgende Aspekte der TCEs beschränken soll:

(a) the products of [creative intellectual activity,] including communal creativity;

(b) indicative of [authenticity/being genuine] of the cultural and social identity and cultural heritage of indigenous peoples and communities and traditional and other cultural communities; and (c) maintained, used or developed by nations, states, indigenous peoples and communities and traditional and other cultural communities, or by individuals having the right or responsibility to do so in accordance with the customary land tenure system or law/customary normative systems or traditional/ancestral practices of those indigenous peoples and communities and traditional and other cultural communities, or has an affiliation with an indigenous/traditional community. ${ }^{6} 96$

\footnotetext{
${ }^{695}$ WIPO IGC, „The Protection of Traditional Cultural Expressions: Draft Articles“, WIPO/GRTKF/IC/19/4, 17. Mai 2011, Art. 1. ${ }^{696}$ Ebd.
} 
Rechtlich geschützte TCEs beschränken sich somit auf die Produkte kreativer geistiger Praxen, die bezeichnend und authentisch für die kulturelle und soziale Identität und das kulturelle Erbe des indigenen Volkes, der Gemeinschaft oder kulturellen Gemeinschaft sind und von dieser Gruppe oder von ihren Mitgliedern bewahrt und genutzt werden oder entwickelt wurden. Absatz 3 stellt des Weiteren klar, dass eine konkrete Auswahl der Begriffe, welche die geschützten Formen bezeichnen, auf nationaler, regionaler oder sub-regionaler Ebene näher bestimmt werden soll. ${ }^{697}$

In der Ausgestaltung des Schutzumfangs ist in den Entwürfen eine gesonderte Ausgestaltung für geheime TCEs vorgesehen, nach welcher den Trägern angemessene und effektive rechtliche und praktische Mittel zur Verfügung gestellt werden sollen ,to prevent any unauthorized fixation, disclosure, use, or other exploitation" von geheimen TCEs. ${ }^{698}$ In der sonstigen Ausgestaltung des Schutzumfangs wird weiterhin deutlich, dass ein Ansatz vergleichbar dem anglo-amerikanischen Urheberrecht angedacht ist, nachdem eine Differenzierung von ökonomischen Rechten und moral rights, also Schutzrechten in Bezug auf die Integrität des Schutzgutes und ihrer Träger, vorgenommen wird. „The economic and moral interests of the beneficiaries of traditional cultural expressions, as defined in Articles 1 and 2, [should] [shall] be safeguarded in a reasonable and balanced manner." ${ }^{\circ 699}$ Der dritte von drei Formulierungsvorschlägen formuliert dies folgendermaßen:

Adequate and effective [legal or practical] measures should be provided to:

1) prevent the [unauthorized fixation, disclosure, use or other exploitation] disclosure of secret traditional cultural expressions;

2) acknowledge the beneficiaries unless this turns out to be impossible;

3) protect against the offensive use of traditional cultural expressions which would be prejudicial to the reputation of the beneficiaries or the integrity of traditional cultural expressions;

4) protect against the use of the non-authentic traditional cultural expressions in trade that suggests a connection that does not exist with beneficiaries; and

5) [where appropriate, ] provide equitable remuneration to the beneficiaries for [[enable] ensure the beneficiaries shall have exclusive and inalienable collective rights to authorize] the following uses of traditional cultural expressions:

\footnotetext{
${ }^{697}$ Ebd.

${ }^{698}$ Ebd., Art. 3 A.

${ }^{699}$ Ebd., Art. 3 B, Alternative 2.
} 
fixation, reproduction, public performance, translation or adaptation, making available or communicating to the public ${ }^{700}$

Neben einem Schutz für geheime TCEs geht es vor allem um den Schutz der Integrität der Ausdrucksformen durch eine Anerkennung der Herkunft, einen Schutz gegen Entstellung und unlautere Verwendung in Produkten, die eine Authentizität vortäuschen. Gleichzeitig soll die Lizenzierung für Nutzungen in Form von Fixierung, Weiterverarbeitung, öffentlichen Aufführungen oder Verbreitung in der Öffentlichkeit gegen eine entsprechende Entlohnung der Träger der TCEs möglich sein. Die Entwürfe sehen auch Regelungen für Ausnahmen und Schranken dieser Rechte vor, so dass beispielsweise gruppenintern keine Beschränkungen der Nutzungen gelten sollen und im Rahmen nationalgesetzlicher Ausgestaltung weitere Ausnahmeregelungen für Archive oder sonstige Regelungen in Übereinstimmung mit dem internationalen Urheberrecht vorgesehen werden können. ${ }^{701}$ Ebenso urheberrechtsähnlich zeigt sich die Ausgestaltung in zeitlicher Hinsicht. So soll für die ökonomischen Rechte eine Begrenzung möglich sein. Für die Rechte bezüglich der kulturellen Integrität sollen jedoch keine Beschränkungen gelten. Die konstitutiven Unterschiede zum Urheberrecht liegen darin, dass die Rechte an TCEs ohne konkrete Fixierung eines Werkes oder vorherige Registrierung gelten sollen. ${ }^{702}$

\section{Traditional Knowledge}

Die Verhandlungen im IGC zum Thema traditional knowledge erwachsen aus einer langjährigen Geschichte, die bisher hauptsächlich im Kontext des internationalen Umweltrechts Anknüpfung gefunden hat, dort aber immer auf den konkreten Bezug zu genetischen Ressourcen beschränkt war. Im IGC findet sich ein umfassenderes Verständnis von TK, welches in Option 2 von Artikel 1 der gegenwärtigen Entwürfe folgendermaßen skizziert wird:

(a) Traditional knowledge is dynamic and evolving. It is the result of the [intellectual activities] in [diverse traditional contexts], including scientific knowledge, skills, competencies, innovations, practices and teachings in a collective framework including codified knowledge systems, continuously developed, evolved and widely used, following any changes in the environment, geographical conditions and other factors [of [indigenous peoples or [and] local communities]];

\footnotetext{
${ }^{700}$ Ebd., Art. 3, Alternative 3.

701 Ebd., Art. 5.

702 Ebd., Art. 7.
} 
Indigenous knowledge of indigenous peoples and indigenous nations must be protected under the principles of the right to self-determination and the right to development.

(b) Traditional knowledge is part of a collective, ancestral, territorial, spiritual, cultural, intellectual and material heritage;

(c) Traditional knowledge is transmitted from generation to generation in diverse forms and is inalienable, indivisible and imprescriptible;

(d) Traditional knowledge is intrinsically linked to biodiversity natural resources and sustains cultural, social and human diversity embodied in traditional lifestyles. ${ }^{703}$

Die Bezüge zu Rechten indigener Völker, dem Recht auf Selbstbestimmung sowie die Bezüge zur biologischen Vielfalt natürlicher Ressourcen verdeutlichen den Hintergrund des Konzepts ,traditionelles Wissen'. Es wird beschrieben als unterschiedliche Arten von Wissensbeständen in traditionellen Kontexten mit einer historischen, intergenerationellen Verwurzelung, welche sich in kollektiver Trägerschaft einer indigenen oder lokalen Gemeinschaft befinden. Das Verständnis von TK im IGC geht damit über die im Umweltrecht bisher normativ verankerte Konkretisierung in Bezug auf genetische Ressourcen weit hinaus.

\section{Zusammenfassung}

Die draft articles neuer Regelungen geistigen Eigentums zu TK/TCEs machen in ihrem jeweils ersten Artikel die unterschiedlichen Gegenstände der Subject Matter of Protection deutlich. In den Verhandlungen werden zudem die beiden Themenbereiche TK und TCE inhaltlich verknüpft, so dass die Struktur und Ausgestaltung der Entwürfe zu den weiteren Fragen der Rechtsdurchsetzung, der Rechtsausübung, Ausnahmen und Grenzen sowie die zeitliche Anwendbarkeit parallel verläuft. Diese Aspekte sollen im Folgenden daher zusammengefasst dargestellt werden.

\section{Durchsetzungsmechanismen}

Die Rechte an TK/TCEs sollen als Rechte geistigen Eigentums privatrechtlich durchgesetzt werden können und auch strafrechtlich abgesichert werden. ${ }^{704}$ Die Rechtsdurchsetzung soll in dem Staat durchgeführt werden, in dem Ansprüche auf Schutz von TK/TCE geltend gemacht werden. Des Weiteren sollen für Streitigkeiten zwischen Berechtigten und Nutzern national und international

\footnotetext{
${ }^{703}$ WIPO IGC, „The Protection of Traditional Knowledge: Draft Articles“, WIPO/GRTKF/IC/19/5, 20. Mai 2011, Annex, S. 1.

${ }^{704}$ WIPO IGC, „The Protection of Traditional Cultural Expressions: Draft Articles“, WIPO/GRTKF/IC/19/4, Art. 8.
} 
anerkannte unabhängige alternative Streitschlichtungsinstanzen zur Verfügung stehen. Im Kern sollen die Staaten sicherstellen, dass

accessible, appropriate and adequate enforcement and disputeresolution mechanisms, border-measures, sanctions and remedies including criminal and civil remedies, should be available in cases of breach of the protection for traditional cultural expressions. ${ }^{705}$

Des Weiteren ist im Rahmen der Regelungen zum Management of Rights zur Unterstützung der Berechtigten die Einrichtung einer national competent authority (NCA), also einer Regierungsbehörde im Heimatstaat der Berechtigten oder eine Institution auf regionaler oder lokaler Ebene, vorgesehen. ${ }^{706}$ Die Berechtigten sollen die NCA zum Handeln ermächtigen dürfen, beziehungsweise soll die NCA auf Anfrage und im Namen der Berechtigten handeln, sofern diese die Unterstützung der Behörde in der Ausübung ihrer Rechte wünschen. Gegenüber den Nutzern von TCEs soll sie dann zuständig sein für folgende Aufgaben:

(a) Grant licenses only after appropriate consultation and with the prior informed consent or approval and involvement of the beneficiaries in accordance with their traditional decision-making and governance processes;

(b) Collect monetary or non-monetary benefits from the use of the traditional cultural expressions providing such benefits shall/should be provided directly by the competent authority to the beneficiaries concerned or utilized for their benefits..$^{707}$

Voraussetzung für die Lizenzierung, so beschreibt dies lit (c) des Entwurfs, ist eine vorausgehende Konsultation und prior informed consent oder eine Einbeziehung der Berechtigten in die Entscheidung unter Beachtung nationaler Regelungen und gewohnheitsrechtlicher Regelungen sowie der traditionellen Entscheidungsfindungsmechanismen der Gruppe. Weiterhin ist angedacht, dass

(d) any monetary [or] and non-monetary benefits collected by the competent authority for the use of the traditional cultural expressions shall/should be provided directly by the designated competent authority to the beneficiaries concerned or utilized

\footnotetext{
705 Ebd., Art. 8, Option 2; Siehe zur vergleichbaren Regelung: WIPO IGC, „The Protection of Traditional Knowledge: Draft Articles“, WIPO/GRTKF/IC/19/5, Art. 4.

706 Für TCEs siehe: WIPO IGC, ,The Protection of Traditional Cultural Expressions: Draft Articles“, WIPO/GRTKF/IC/19/4, Art. 4; Für TK siehe WIPO IGC, ,The Protection of Traditional Knowledge: Draft Articles“, WIPO/GRTKF/IC/19/5, Art. 5.

707 WIPO IGC, „,The Protection of Traditional Cultural Expressions: Draft Articles“, WIPO/GRTKF/IC/19/4, Art. 4, Abs. 1.
} 
[for their benefits] for the direct benefit of relevant beneficiaries and the preservation of traditional cultural expressions. ${ }^{708}$

Die NCA nimmt also neben der Lizenzvergabe auch die Verwaltung der finanziellen und nicht-finanziellen Vorteile im Sinne der Berechtigten wahr. Zusätzlich soll die NCA Aufklärungsarbeit bei den Berechtigten leisten und diese insbesondere bei eigenen Verhandlungen mit Nutzern umfassend unterstützen sowie die Nutzung der TCEs überwachen. ${ }^{709}$

Im Kontext TK soll die NCA vergleichbare Aufgaben wahrnehmen und so unter anderem Informationen für die Berechtigten zur Verfügung stellen, sicherstellen, ob die Regelungen zum prior informed consent beachtet wurden, die faire und gerechte Vorteilsteilhabe überwachen, die Rechtsausübung unterstützen oder übernehmen sowie Verletzungen feststellen. ${ }^{710}$

\section{Zusammenfassung}

Die Initiativen des globalen Südens zum Schutz traditioneller kultureller Ausdrucksformen durch Instrumente geistigen Eigentums führten nach einer über 35-jährigen Vorgeschichte zu den gegenwärtigen Verhandlungen im IGC der WIPO. Sie werden dort seit 2001 zusammen mit den Themenbereichen TK und GR diskutiert. Dabei zeichnen sich die gegenüberstehenden Verhandlungspositionen deutlich ab: Auf der einen Seite bemühen sich die GRULAC, die afrikanische Gruppe sowie die überregionale Gruppe der like-minded countries um neue Schutzinstrumente für kulturelle Ausdrucksformen und traditionelles Wissen, da es in ihrem Interesse ist, diese Werte vor der zunehmenden nichtberechtigten Nutzung und Kommerzialisierung zu schützen und Regelungen einer gerechten Vorteilsteilhabe zu etablieren. Auf der anderen Seite stehen die westlichen Staaten, welche die Verhandlungen verlangsamen, um keine Kompromisse schließen zu müssen. Gleichzeitig binden sie die Verhandlungen zu GR/TK/TCEs in der WIPO und entziehen diesen Themenbereich somit den Diskussionen in anderen Foren, insbesondere der WTO, der UNESCO und der CBD. Das bestehende System der Rechte geistigen Eigentums arbeitet in ihrem Interesse, da es technisches Wissen durch das Patentrecht sowie verkörperte geistige Leistungen durch das Urheberrecht schützt und in dieser Form den in ihren Staaten etablierten Wirtschaftsbereichen der Pharma- und Medienindustrie nützt. Andere geistige Leistungen, die über große Zeiträume hinweg in den Ländern des globalen Südens bewahrt und entwickelt wurden, werden hingegen rechtlich nicht geschützt

\footnotetext{
708 Ebd.

709 Ebd., Art. 4, Abs. 2.

710 WIPO IGC, „The Protection of Traditional Knowledge: Draft Articles“, WIPO/GRTKF/IC/19/5, Art. 5.
} 
und bleiben in der public domain ohne zusätzliche Kosten einer Lizenzierung verfügbar.

Die Entwicklungen zum rechtlichen Schutz traditionellen Wissens und traditioneller kultureller Ausdrucksformen wie sie sich gegenwärtig in den draft articles des WIPO IGC abzeichnen, weisen erhebliche Parallelen zum Urheberrecht auf. Insbesondere die privatrechtliche Ausgestaltung der Lizenzierung von Nutzungsrechten Dritter sowie die Unterteilung in economic und moral rights machen dies deutlich. Für TK/TCEs ergeben sich jedoch im Vergleich zum copyright Unterschiede in der kollektivrechtlichen Ausgestaltung der Berechtigten und der explizit nicht notwendigen Fixierung des Schutzgegenstandes. Diese Neuerungen sollen die bisherigen Probleme bei der Anwendung des Urheberrechts zum Schutz traditioneller kultureller Ausdrucksformen überwinden und damit neue Möglichkeiten eröffnen, einen umfassenderen rechtlichen Schutz für TK/TCEs gewähren zu können. 


\section{B. Die beneficiaries neuer Instrumente zum Schutz von TK/TCEs}

Eines der kennzeichnenden Merkmale neuer Rechtsinstrumente geistigen Eigentums an traditionellem Wissen und traditionellen kulturellen Ausdrucksformen, wie sie im WIPO IGC diskutiert werden, ist die Ausgestaltung als Kollektivrecht. Ausgehend von der Prämisse, dass die kulturellen Wurzeln von TK/TCEs von Kollektiven getragen werden, sollen diese Kollektive entsprechend als Berechtigte anerkannt und normiert werden. In Anbetracht der Komplikationen der rechtstechnischen Ausübung einer kollektiven Rechtsträgerschaft differenzieren die draft articles zwischen beneficiaries (Artikel 2) und dem management of rights (Artikel 4). Es zeigen sich in den Verhandlungen zur Frage der beneficiaries die hintergründigen Vorstellungen, wie sich die verhandelnden Staatenvertreter aus völkerrechtlicher Perspektive das Neben- und Miteinander kulturell distinkter Gruppen in ihren Heimatländern vorstellen.

\section{Die draft articles}

Die draft articles normierten in unterschiedlichen Fassungen verschiedene Begrif$\mathrm{fe}$, welche die beneficiaries bestimmen sollten. Sie bezogen sich sowohl auf die etablierten Konzepte der ,Minderheiten' und ,indigenen Völker' sowie auf das Begriffspaar der, indigenen und lokalen Gemeinschaften' aus dem Umweltvölkerrecht. Des Weiteren wurde die Verwendung neuer Begriffe verhandelt. Die Beschreibung der beneficiaries in Artikel 2 der Entwürfe zu Rechten und TCEs wurde zum Ende des fünften Bianniums 2011 in folgenden drei Optionen diskutiert:

\section{Option 1}

Beneficiaries of protection for traditional cultural expressions, as defined in Article 1, are indigenous peoples/communities and local communities, who develop, use, hold and maintain the cultural expressions.

Option 2

Beneficiaries of protection of traditional cultural expressions, as defined in Article 1, are the holders of traditional cultural expressions which may include:

(a) Indigenous communities; (b) local communities; (c) traditional communities; (d) cultural communities; (e) families; (f) nations; (g) individuals within the categories listed above; and (h) where traditional cultural expressions are not specifically attributable to or confined to an indigenous or local community or it is not 
possible to identify the community that generated it, any national entity determined by domestic law.

\section{Option 3}

Beneficiaries of protection for traditional cultural expressions, as defined under Article 1, are indigenous peoples, local and traditional communities, including small-island states. ${ }^{711}$

Artikel 2. der draft articles zu traditionellem Wissen formulierte Anfang 2012 folgende zwei Optionen:

\section{Option 1}

Beneficiaries of protection of traditional knowledge, as defined in Article 1, are indigenous peoples/communities and local communities.

\section{Option 2}

Beneficiaries of protection of traditional knowledge, as defined in Article 1, may include:

(a) indigenous peoples/communities; (b) local communities; (c) traditional communities; (d) families; (e) nations; (f) individuals within the categories listed above; and (g) where traditional knowledge is not specifically attributable or confined to an indigenous peoples or local community, or it is not possible to identify the community that generated it, any national entity determined by domestic law. ${ }^{712}$

Die Entwürfe zur Normierung der beneficiaries reichten von Individuen über Familien und Stämme, kulturelle, traditionelle, lokale und indigene Gemeinschaften sowie indigene Völker bis hin zu Nationen. In den Verhandlungen wurde auch die Verwendung des Begriffs minority vorgeschlagen. ${ }^{713}$ Konkrete Definitionen zu den Bedeutungen dieser Begriffe wurden jedoch nicht in die draft articles eingebracht. Und auch die Kommentierungen mit weiteren Ausführungen und Bezügen zu Verhandlungspositionen der draft articles und den frühe-

711 WIPO Generalversammlung, ,Matters Concerning the Intergovernmental Committee on Intellectual Property and Genetic Resources, Traditional Knowledge and Folklore (IGC)“, WO/GA/40/7, Annex A, Art. 2.

712 WIPO IGC, „The Protection of Traditional Knowledge: Draft Articles“, WIPO/GRTKF/IC/21/4, 18. Januar 2012, Art. 2.

713 So brachte beispielsweise China während der 17. Sitzung einen entsprechenden Vorschlag ein. WIPO IGC, „Seventeenth Session, December 6 to 10, Report“, WIPO/GRTKF/IC/17/12, Abs. 109; Auch in früheren Versionen der draft articles fand der Begriff Verwendung. WIPO IGC, ,The Protection of Traditional Cultural Expressions: Draft Articles“, WIPO/GRTKF/IC/19/4, Art. 2. Die Bezugnahme zum Konzept minorities geschieht allerdings nur vereinzelt, so dass dessen Verwendung im Folgenden nicht im Detail nachgegangen werden soll. 
ren revised objectives and principles, die den einzelnen Artikeln jeweils von Seiten des Sekretariats beigefügt wurden, enthalten keine näheren Definitionen der verwendeten Terminologie.

Allerdings erläutert das vom IGC Sekretariat erstellte Glossar die Bedeutung und Definitionen einiger der verwendeten Begriffe. Der Glossar wurde während der 16. Sitzung für den Themenbereich der TCEs in Auftrag gegeben, um als Informationsgrundlage zu den meistgenutzten Begriffen zu dienen. Entsprechende Leitfäden wurden auch für TK und GR entwickelt und schließlich nach der 20. Sitzung in einem kohärenten Dokument zusammengeführt, welches nun das Glossar für den gesamten Verhandlungsgegenstand des IGCs bildet. ${ }^{714}$

Neben den völkerrechtlich bereits geprägten Konzepten ,indigenous peoples ' und ,indigenous and local communities' sowie den selbsterklärenden Bezeichnungen individuals und families werden die neuen Begriffe der ,traditional" und ,cultural community" verwendet. ${ }^{715}$ Streitig verhandelt wurde die Bedeutung des Begriffs nation sowie die damit einhergehende Rolle des Staates bei der näheren Bestimmung der Berechtigten. ${ }^{716}$ Im Folgenden soll zunächst der Gebrauch der etablierten Begrifflichkeiten sowie die Diskussion um die Bedeutung des Begriffs nation skizziert werden, bevor die neuen Begrifflichkeiten verortet werden können.

\section{Verwendung etablierter Konzepte substaatlicher Gruppen}

Im Zentrum der Debatten zur Bestimmung der Berechtigten neuer Gruppenrechtsinstrumente für TK und TCEs stehen die bereits etablierten Konzepte der ,indigenen Völker'sowie der, indigenen und lokalen Gemeinschaften‘. Dies zeigt

714 WIPO IGC, „Nineteenth Session, July 18 to 22, 2011, Report“, WIPO/GRTKF/IC/19/12, Abs. 174; WIPO IGC, „Glossar“, WIPO/GRTKF/IC/20/INF/13.

715 Der Vorschlag zur Einbeziehung von Individuen und Familien stammt von der arabischen Gruppe. WIPO IGC, „Eighteenth Session, May 9 to 13, 2011, Report“,

WIPO/GRTKF/IC/18/11. Er findet jedoch nur vereinzelt Zustimmung. Die Kommentierung der Revised Objectives and Principles führt dazu aus: „As discussed in relation to Article 1, these provisions are intended primarily to benefit communities, including in cases where a TCE/EoF is created or developed by an individual member of a community. The essential characteristics of 'traditional' creations are that they contain motifs, a style or other items that are characteristic of and identify a tradition and a community that still bears and practices it. Thus, even where an individual has developed a tradition-based creation within his or her customary context, it is regarded from a community perspective as the product of social and communal creative processes. The creation is, therefore, not 'owned' by the individual but 'controlled' by the community, according to indigenous and customary legal systems and practices. This is what marks such a creation as 'traditional'. "WIPO IGC, „The Protection of Traditional Cultural Expressions/Expressions of Folklore: Revised Objectives and Principles", WIPO/GRTKF/IC/17/4, Art. 2, Commentary, S. 22; Obwohl Familien und Clans auch mehrere Hundert Mitglieder umfassen können, findet die Einbeziehung dieser als Berechtigte keine verbreitete Zustimmung.

716 WIPO Generalversammlung, „Matters Concerning the Intergovernmental Committee on Intellectual Property and Genetic Resources, Traditional Knowledge and Folklore (IGC)“, WO/GA/40/7, Annex A, Art. 2 Commentary as prepared by the facilitator, S. 8. 
sich sowohl im Vergleich der unterschiedlichen Versionen der draft articles als auch in den Debatten während Sitzungen des IGC. Der Bericht des IGC zur WIPO Generalversammlung nach der 22. Sitzung bezieht sich für die beneficiaries von TCEs nur noch auf die bereits etablierten Konzepte, indigenous peoples' und ,local communities', nachdem in früheren Fassungen auch eine Reihe andere Begriffe eine Rolle gespielt hat. ${ }^{717}$ In Anbetracht der mangelnden positivrechtlichen Definitionen dieser Konzepte wird im Zuge der Verhandlungen auch deren Bedeutung weiterführend verhandelt.

\section{1. ,Indigenous Peoples/Communities‘}

Bei der Verwendung des Konzepts, indigene Völker ${ }^{\varsigma}$ treten die bekannten Probleme der mangelnden allgemein anerkannten Definition sowie der politischen Implikationen des Anspruchs auf Selbstbestimmung zu Tage. Im Glossar wird deutlich gemacht, dass

[t]he term 'indigenous peoples' has been the subject of considerable discussion and study. There is no universal, standard definition of 'indigenous peoples'. ${ }^{718}$

Außerdem äußerten Staatenvertreter während der Verhandlungen, dass sie zwar die Anliegen indigener Völker unterstützen, dass das Konzept der ,indigenen Völker' in ihren Ländern jedoch keine Anwendung fände. ${ }^{719}$ Die Problematik fasste die Delegation aus Oman folgendermaßen zusammen:

The Delegation of Oman did not think that the term 'indigenous peoples' was applicable to all countries. There were some countries in which there were no Indigenous peoples as such.

Therefore, it suggested using terminology which would take into account the various and diverse situations. It believed that there

\footnotetext{
717 WIPO Generalversammlung, „Matters Concerning the Intergovernmental Committee on Intellectual Property and Genetic Resources, Traditional Knowledge and Folklore (IGC)“, WO/GA/41/15, 1. August 2012, Annex C, S. 7.

718 WIPO IGC, „Glossar“, WIPO/GRTKF/IC/20/INF/13, Annex, Stichwort „Indigenous Peoples".

${ }^{719}$ So äußerte sich der Verteter Chinas beispielsweise folgendermaßen: „The Delegation of China expressed its support, and endorsement to the reasonable appeals of the Indigenous people in respect of TK and GRs although the concept of ,indigenous people $e^{6}$ was not applicable in China.“ WIPO IGC, „Eighteenth Session, May 9 to 13, 2011, Report“, WIPO/GRTKF/IC/18/11, Abs. 108; Ähnlich äußerte sich die Delegation Ägyptens: „The Delegation of Egypt stated that, despite the fact that Egypt did not have indigenous nations as such, it supported the idea of protection being given to the rights of indigenous peoples to protect and preserve their culture and cultural heritage. [...] Among the definitions, there had to be a reference to,indigenous peoples and indigenous communities“" WIPO IGC, „Seventeenth Session, December 6 to 10, 2010, Report“",WIPO/GRTKF/IC/17/12, Abs. 114; Auch Marokko äußerte sich entsprechend. Ebd., Abs. 122.
} 
were only two terms which could be used: 'peoples' and 'nations'. It believed that those would be satisfactory to all parties concerned. ${ }^{720}$

Vor dem Hintergrund der politischen Implikationen des Begriffs ,indigene Völker' und seiner geographisch differenzierten Anwendung von Seiten der Staaten wurden die alternativen Begriffe, indigenous nations' und ,indigenous communities" vorgeschlagen. So äußerte beispielsweise der Vertreter Bangladeshs im Namen der asiatischen Gruppe ,its preference for a definition of 'beneficiaries' in Article 2 that would encompass indigenous communities, among others [sic!]. The Delegation had difficulties in accepting a term that would restrict the scope of beneficiaries. "721 Die Kommentierung zu Artikel 2 der draft articles fasst den Verhandlungsstand folgendermaßen zusammen:

The proponents of the more limited definition of beneficiaries reflected in Option 1 had different views on whether to refer to 'indigenous peoples' or 'indigenous communities'. As a placeholder, acknowledging that it was a matter that required further work to resolve, the facilitator referred to indigenous peoples/communities' in Option $1 . .^{722}$

Zur Begriffsklärung weist das Glossar für den Begriff ,indigenous peoples 'zunächst auf die Arbeitsdefinition des UN Sonderberichterstatters José Martínez Cobo und seine allgemeine Anerkennung auch bei Vertretern indigener Völker hin. ${ }^{723}$ Des Weiteren wird ausgeführt, dass der Begriff ebenfalls im UNEP Glossary of Biodiversity Terms, bei der Weltbank, dem Indigenous Department der ILO und in der kanadischen Verfassung Verwendung fände. Letztgenannte normiere beispielsweise folgende Definition:

Aboriginal Peoples of Canada includes the Indian, Inuit and Metis peoples of Canada. The 1996 Canadian Royal Commission on Aboriginal People self-defined their focus group as: ,... organic political and cultural entities that stem historically from the original peoples of North America $[\ldots]^{6} .^{724}$

\footnotetext{
720 WIPO IGC, „Eighteenth Session, May 9 to 13, 2011, Report“, WIPO/GRTKF/IC/18/11, Abs. 111.

721 WIPO IGC, „Seventeenth Session, December 6 to 10, 2010, Report“, WIPO/GRTKF/IC/17/12, Abs. 31.

722 WIPO Generalversammlung, „Matters Concerning the Intergovernmental Committee on Intellectual Property and Genetic Resources, Traditional Knowledge and Folklore (IGC)“, WO/GA/40/7, Annex A, Art. 2 Commentary as prepared by the facilitator, S. 8.

${ }^{723}$ WIPO IGC, „Glossar“", WIPO/GRTKF/IC/20/INF/13, Annex, Stichwort „Indigenous Peoples“.

${ }^{724}$ Ebd.
} 
Zur Beschreibung des Begriffs ,indigenous peoples 'wird im Glossar somit das klassische Bild indigener Völker gezeichnet, ohne dabei die neueren Entwicklungen zur Anwendung in einem modernen Verständnis und seine Verwendung in Afrika und Asien deutlich zu machen. Auch die Äußerungen von Staatenvertretern, in denen diese sich gegen die Anwendbarkeit des Konzepts in ihren Staaten aussprechen, zeigen, dass sie von einer auf die typischen Siedlerstaaten beschränkten Bedeutung ausgehen. Gleichzeitig äußern die Staatenvertreter ihre Unterstützung für die Anliegen indigener Völker. Sie entwickeln somit eine Rhetorik, die ihre Position mit den Anliegen der Indigenen verknüpft und eine gemeinsame Verhandlungsposition darstellen soll. Eine Anerkennung des Konzepts für das eigene Land bleibt jedoch aus.

\section{2. ,Local Communities'}

Die Verwendung des Begriffs, local community', wie er sich im Umweltrechtskontext als Alternative zum Konzept der, indigenen Völker' entwickelt hat, erfährt in den Verhandlungen des IGC teilweise eine Öffnung und Neuausrichtung. Dazu löste er sich aus seiner Verwendung als stehendes Begriffspaar von den ,indigenous communities ' und wird zusammen mit diesem in unterschiedlichen Variationen und als alleinstehender Begriff verwendet. Ausgangspunkt einer Begriffsbestimmung im IGC ist dennoch seine Verwendung im Umweltrecht. So beschreibt die erste Fassung des Glossars zu TCEs den Begriff, local community' in seiner Verwendung in der CBD. Dort ist er definiert

as the human population in a distinct ecological area who depend directly on its biodiversity and ecosystem goods and services for all or part of their livelihood and who have developed or acquired traditional knowledge as a result of this dependence, including farmers, fisherfolk, pastoralists, forest dwellers and others. ${ }^{725}$

In dieser Beschreibung des Begriffs wird die Beziehung zu umweltrechtlichen Regelungen deutlich und beschreibt die Gruppen mit typischen Berufsgruppen: Bauern, Fischer, Viehhalter, Waldbewohner.

Zum Begriffspaar der ,indigenous and local communities 'wird im Glossar neben der Feststellung, dass keine universelle, standardisierte Definition existiere, auf seine Verwendung in $\operatorname{der} C B D$, in recognition of communities that have a long association with the lands and waters that they have traditionally lived on or used" sowie auf den Gebrauch im Nagoya Protokoll, dem FAO International Treaty on Plant Genetic

725 WIPO IGC, „Glossary of Key Terms Related to Intellectual Property and Traditional Cultural Expressions“, WIPO/GRTKF/IC/19/INF/7, 17. Mai 2011, Annex, Stichwort „Local Community“. 
Resources for Food and Agriculture und dem Swakopmund Protocol verwiesen. ${ }^{726}$ Ebenso wird festgehalten, dass

Article 1 of the Decision 391 on Access to Genetic Resources of Andean Community defines 'native, Afro-American or local community' as 'a human group whose social, cultural and economic conditions distinguish it from other sectors of the national community, that is governed totally or partially by its own customs or traditions or by special legislation and that, irrespective of its legal status, conserves its own social, economic, cultural and political institutions or a part of them.'

Article 7.III of the Brazilian Provisional Act No. 2,186-16, dated August 23, 2001, defines 'local community' as a 'human group, including descendants of Quilombo communities, differentiated by its cultural conditions, which is, traditionally, organized along successive generations and with its own customs, and preserves its social and economic institutions. ${ }^{727}$

Zur Bestimmung des Begriffs wird zunächst auf seine Verwendung im umweltrechtlichen Kontext auf internationaler Ebene verwiesen. Außerdem wird auf die nationalen Formen der Ausgestaltung verwiesen, die auf eine kulturelle Distinktion in Bezug auf Gebräuche, Tradition und eigene soziale, wirtschaftliche, ökonomische und kulturelle Institutionen abstellen. Eine weitergehende Prägung oder Auslegung wird jedoch nicht vorgenommen und somit die Kontextualisierung des Begriffspaares, indigene und lokale Gemeinschaften' im Bereich der nachhaltigen Lebensweise, der biologischen Vielfalt und dem traditionellen Wissen in Bezug auf genetische Ressourcen bestätigt.

Von Seiten der Staatenvertreter werden neue Impulse in die Diskussion eingebracht. So zeigt die Verwendung des Begriffs, local community" in den Vorlagen zu TK und TCE der Gruppe der like-minded countries einerseits eine allgemeine, über das Umweltrecht hinausgehende Offenheit sowie andererseits Verweise zur näheren Konkretisierung auf nationaler Ebene.

For the purposes of this article, the term 'local communities' shall include any classification of social and cultural identity of a member state as defined by domestic law. ${ }^{728}$

\footnotetext{
726 WIPO IGC, „Glossar“, WIPO/GRTKF/IC/20/INF/13, Annex, Stichwort „Indigenous and Local Community“.

727 Ebd.

728 Ebd.
} 
Ähnlich äußerte sich der Vertreter Chinas mit seiner Forderung, ,there should be sufficient flexibilities in the meaning of 'local communities" ". ${ }^{729}$ Und auch der Kommentar zu den draft articles stellt fest, dass

[t]here were also different views on whether to also refer to 'traditional' or 'cultural' communities. The facilitator left these out of the draft, on the understanding that further work was needed on the definition of these terms, as well as concerning the term 'local communities' and what it encompasses. ${ }^{730}$

Trotz der bisherigen Prägung des Konzepts, local community 'im Umweltvölkerrecht wird in den Verhandlungen im IGC somit von einem offenen Verständnis ausgegangen, welches über den Kontext der Nachhaltigkeit und Biodiversität hinausgeht.

III. Diskussionen um die Berechtigung der ,Nation` und die Rolle des Staates

Die Diskussionen in den Sitzungen des IGC zur Frage der beneficiaries befassten sich neben etablierten Konzepten substaatlicher Gruppen auch damit, ob ,nations' als Berechtigte genannt werden sollten. Für Staaten, die vertreten in ihrer Zusammensetzung aus nur einer homogenen Gruppe zu bestehen, ergäbe sich damit eine Interpretationsmöglichkeit, nach der die Berechtigten eines entsprechenden Instruments mit dem Staatsvolk gleichzusetzen wären. Die Normierung der Rechte an TK und TCEs in Bezug auf Staaten als Berechtigte führte jedoch zu Kritik. So äußerte beispielsweise die Delegation der USA ihre Bedenken,

because it was troubled by the word 'nation'. It agreed with the representative of CISA [einer NGO, die sich dazu entsprechend geäußert hat] that if 'nation' meant Indigenous people or nation of Indigenous people, it would not be troubled. But "nation" might mean 'Nation State'. It was cognizant of the point made by the Delegation of Barbados, and it supported the idea that small nations and small island nations might constitute communities. ${ }^{731}$

\footnotetext{
${ }^{729}$ WIPO IGC, „Eighteenth Session, May 9 to 13, 2011, Report“, WIPO/GRTKF/IC/18/11, Abs. 109.

730 WIPO Generalversammlung, „Matters Concerning the Intergovernmental Committee on Intellectual Property and Genetic Resources, Traditional Knowledge and Folklore (IGC)“, WO/GA/40/7, Annex A, Art. 2, Commentary as prepared by the facilitator, S. 8.

731 WIPO IGC, „Eighteenth Session, May 9 to 13, 2011, Report“", WIPO/GRTKF/IC/18/11, Abs. 93.
} 
Hintergrund dieser Aussage war die Forderung Barbados', unterstützt von anderen Kleinststaaten, ${ }^{732}$ den Begriff ,nation‘ als Berechtigte aufzunehmen.

Barbados stated that it did not have identified Indigenous people and the society was not categorized according to communities. TK belonged to the Barbadian society as a whole. The only word in that article which would accommodate its interests was the word 'nations'. Therefore, it supported the inclusion of 'nations' as a beneficiary. ${ }^{733}$

Neben den Kleinst- und Inselstaaten sind es allerdings vorrangig die Vertreter afrikanischer und asiatischer Staaten, die für die Verwendung des Begriffs ,nation' eintreten. Die Delegation Südafrikas setzte sich beispielsweise in der 17. Sitzung im Namen der Afrikanischen Gruppe dafür ein, den ersten Teil des Artikel 2 auf ,indigenous peoples, communities and nations“ zu kürzen, wobei „communities“ in einer Fußnote weitere Konkretisierung erfahren sollte. ${ }^{734}$ Algerien unterstützt den Wortlaut der zweiten Intersessional Workinggroup (,indigenous peoples, local communities [and nations]") mit der Verwendung des Begriffs ,nation', da dieser flexibel genug sei, um unterschiedliche Situationen in verschiedenen Ländern abzubilden. ${ }^{735} \mathrm{Im}$ Kommentar zu den revised objectives and principles ist vermerkt, dass ,the African Group has stated that principles for the protection of TCEs/EoF should 'Recognize the role of the State in the preservation and protection of traditional knowledge and expressions of folklore" .736 Auch China erkennt zwar einerseits die Ansprüche indigener Völker an, macht aber andererseits deutlich, dass das Konzept ,indigener Völker' in China nicht anwendbar sei. Die Dokumente müssten somit die Begriffe ,Nationen', ,Staat' und Konzepte, wie beispielsweise ,Minderheiten' enthalten. ${ }^{737}$

Ebenso unterstützen Indonesien und Sri Lanka explizit die Forderung, den Begriff ,nations' zu verwenden. ${ }^{738}$ Die Delegation Thailands machte ebenfalls deutlich, dass sie diese Formulierung unterstützt:

\footnotetext{
732 Ebd., Trinidad und Tobago: Abs. 109; Ebd., Saint Kitts and Nevis: Abs. 114.

733 WIPO IGC, „Eighteenth Session, May 9 to 13, 2011, Report“, WIPO/GRTKF/IC/18/11, Abs. 91.

734 WIPO IGC, ,Seventeenth Session, December 6 to 10, 2010, Report“", WIPO/GRTKF/IC/17/12, Abs. 106.

735 WIPO IGC, „Eighteenth Session, May 9 to 13, 2011, Report“, WIPO/GRTKF/IC/18/11, Abs. 82

736 WIPO IGC, „The Protection of Traditional Cultural Expressions/Expressions of Folklore: Revised Objectives and Principles", WIPO/GRTKF/IC/17/4, S. 21.

737 WIPO IGC, „Seventeenth Session, December 6 to 10, 2010, Report“, WIPO/GRTKF/IC/17/12, Abs. 109; WIPO IGC, „Eighteenth Session, May 9 to 13, 2011, Report“, WIPO/GRTKF/IC/18/11, Abs. 108.

738 WIPO IGC, „Eighteenth Session, May 9 to 13, 2011, Report“, WIPO/GRTKF/IC/18/11, Indonesien: Abs. 76; Sri Lanka: Abs. 81.
} 
[I] t had opened the door to include other communities, including nations which meant nations and communities. In certain countries, circumstances, and developments had transcended the TK of local communities into that of nation communities. It subscribed to the proposal made by the Delegation of Indonesia to make sure that the word 'nations' was included. ${ }^{739}$

Schließlich findet sich in den revised objectives and principles der Vorschlag Indonesiens, dass die Definition der Berechtigten die folgenden Elemente enthalten solle:

(i) other than traditional/indigenous communities as parties who maintained and developed TCE/EoF, governments also needed to play a role in facilitating TCE/EoF protection in case there were other communities who had potential benefits for the utilization of TCE/EoF;

(ii) in cases where the owner of TCE/EoF could not be identified, the beneficiary of TCE/EoF protection should be the government, such as the local government, and the TCE/EoF would be used for the sake of community's interests;

(iii) the owner of $\mathrm{TCE} / \mathrm{EoF}$ eligible to benefit from the protection should be the TCE/EoF owner who had been identified by the local government; [...]

(v) a state could play a certain role in facilitating the protection of the community and it could be extended further as a right holder only if it benefited the communities. ${ }^{740}$

Die Asiatische Gruppe setzt sich somit ausdrücklich für eine Ausgestaltung ein, in der die Nation als Berechtigter anerkennt wird und der Staat eine entscheidende Rolle bei der Auswahl substaatlicher Gruppen als Berechtigte spielen soll.

Uneinheitlich sind die Aussagen der GRULAC zu diesem Thema. Neben den Kleinst- und Inselstaaten spricht sich beispielsweise Bolivien für die Verwendung des Begriffs ,nation“ aus und schlägt folgende Formulierung vor: „Beneficiaries of protection include indigenous peoples, local communities and nations, in accordance with national legislation. " 741 Kolumbien hingegen ,did not agree to include either ,nations` or ,States'،"742 Ecuador schlägt vor, die Begrifflichkeit ,nations‘ im

\footnotetext{
739 WIPO IGC, „Eighteenth Session, May 9 to 13, 2011, Report“, WIPO/GRTKF/IC/18/11, Abs. 101.

740 WIPO IGC, „The Protection of Traditional Cultural Expressions/Expressions of Folklore: Revised Objectives and Principles", WIPO/GRTKF/IC/17/4, S. 22.

741 WIPO IGC, „Eighteenth Session, May 9 to 13, 2011, Report“, WIPO/GRTKF/IC/18/11, Abs. 89.

742 Ebd., Abs. 94.
} 
Glossar durch das Sekretariat verdeutlichen zu lassen. ${ }^{743}$ Uruguay weist darauf hin, dass die Verwendung der Begriffe, nation' und ,states' notwendig sei, um keine Staaten auszuschließen, die, wie beispielsweise Uruguay selbst, keine indigenen Völker beheimateten. ${ }^{744}$

Die Staaten der Gruppe B hielten sich bei dieser Diskussion mit konkreten Forderungen zurück. Es äußerte sich die Delegation Australiens, dass indigene Gemeinschaften die Hauptberechtigten sein sollten und das ,indigenous and local communities could be inclusive of nations in some circumstances." ${ }^{\text {"7 }} 45$

Neben der eingangs dargestellten Kritik der USA widersprechen auch die Vertreter der NGOs einer Stärkung der Rolle der Staaten. Empört äußerte sich beispielsweise der Vertreter der Tupaj Amary: ,[T] he text was unacceptable. The ideas, notions and concepts such as ,nations' had never been raised before [by] any legal specialist.“" 746 Des Weiteren wird kritisiert, dass Rechte indigener Völker dem nationalen Recht unterstellt werden sollen. ${ }^{747}$

Zusammenfassend lässt sich feststellen, dass die Normierung von nations als Berechtigte neuer Rechte an TK und TCEs sowie die damit verknüpfte Debatte zur Rolle des Staates bei der näheren Bestimmung der Berechtigten auf nationaler Ebene insbesondere von der asiatischen und der afrikanischen Gruppe sowie von den Kleinst- und Inselstaaten unterstützt wird. Mitglieder von Teilen der GRULAC, der Gruppe B und die Vertreter indigener Gemeinschaften widersprechen diesen Forderungen. Ein Kompromiss zeichnet sich für die Kleinst- und Inselstaaten ab, die mit dem Argument überzeugen können, ihre geringe Bevölkerungszahl lasse sich nicht weiter in kulturell distinkte Subgruppen unterteilen. So findet die Bezeichnung nation in Option 3 der draft articles keine Verwendung, während Kleinstund Inselstaaten explizit genannt werden. ${ }^{748}$ Diese Auslegung wird auch aus den Verhandlungen deutlich, sodass Kleinst- und Inselstaaten durch die Gruppe ihres Staatsvolks als Nation als Berechtigte neuer Instrumente für TK/TCEs gelten können, während dies für größere Staaten weiterhin umstritten bleibt.

\footnotetext{
743 Ebd., Abs. 99.

744 WIPO IGC, „Nineteenth Session, July 18 to 22, 2011, Report“, WIPO/GRTKF/IC/19/12, Abs. 79.

745 WIPO IGC, „Eighteenth Session, May 9 to 13, 2011, Report“, WIPO/GRTKF/IC/18/11, Abs. 77.

746 Ebd., Abs. 110.

747 WIPO IGC, „Seventeenth Session, December 6 to 10, 2010, Report“, WIPO/GRTKF/IC/17/12, Abs. 37.

748 WIPO Generalversammlung, „,Matters Concerning the Intergovernmental Committee on Intellectual Property and Genetic Resources, Traditional Knowledge and Folklore (IGC)“, WO/GA/40/7, Annex A, Art. 2, Option 3.
} 
IV. Neue Konzepte: ,Traditional Communities' und ,Cultural Communities`

Neben den Konzepten der ,indigenen Völker', der ,indigenen und lokalen Gemeinschaften' und der ,Nation' wurden auch neue Begrifflichkeiten im Verlauf der Verhandlungen diskutiert und zeitweilig in den draft articles verwendet: ,traditional communities' und, cultural communities'.

Der Begriff ,traditional community 'wird im Glossar nicht mit einem eigenen Eintrag definiert. Nur im Kontext der Erklärung der ,indigenous and local communities" wird auf seine Verwendung im Swakopmund Protocol on the Protection of Traditional Knowledge and Expressions of Folklore hingewiesen. Außerdem findet er in der Beschreibung des Konzepts traditional knowledge Verwendung, wo er als Oberbegriff für ,indigenous and local communities ' dient. ${ }^{749} \mathrm{Im}$ Kommentar der Vermittlerin zu den draft articles zum Schutz von TK wird vorgeschlagen, den Begriff als vom Konzept der , local community " umfasst zu verstehen und entsprechend aus den Entwürfen zu streichen. ${ }^{750}$ Aus den Verhandlungen des IGC ergibt sich insgesamt keine weiterführende Klärung der Bedeutung des Konzepts ,traditional community!

Hingegen hat der Begriff , cultural communities 'im Glossar und in den Kommentaren zu den draft articles eine nähere Beschreibung erfahren. Im Glossar wird unter Bezugnahme auf einen Vorschlag einer niederländischen Expertengruppe folgendes ausgeführt:

'Cultural community' has been defined as a tightly knit social unit whose members experience strong feelings of unity and solidarity and which is distinguished from other communities by its own culture or cultural design, or by a variant of the generic culture. ${ }^{751}$

Eine ,cultural community' bestimmt sich demnach durch ein Zusammengehörigkeitsgefühl ihrer Mitglieder und durch eine kulturelle Distinktion gegenüber anderen Gemeinschaften. Diese Beschreibung des Begriffs ,cultural community ‘ normiert damit den Kerngehalt ähnlich dem der Definitionsansätze der etablierten Konzepte. ${ }^{752}$ Sie stellt auf die Elemente der Zusammengehörigkeit in einer kulturellen Distinktion gegenüber Anderen ab, ohne weitere einschränkende

${ }^{749}$ WIPO IGC, „Glossar“, WIPO/GRTKF/IC/20/INF/13, Annex, Stichwort „Traditional Knowledge".

750 WIPO Generalversammlung, „Matters Concerning the Intergovernmental Committee on Intellectual Property and Genetic Resources, Traditional Knowledge and Folklore (IGC)“, WO/GA/41/15, Annex B, S. 31.

751 WIPO IGC, „Glossar“, WIPO/GRTKF/IC/20/INF/13, Annex, Stichwort „Cultural Community“. Eine Fußnote verweist dazu auf: UNESCO, International Meeting of Experts on Intangible Cultural Heritage, „Draft Glossary proposed by a group of Dutch experts convened by the bureau of the Netherlands National Commission for UNESCO“, TER/CH/2002/WD/4, 6. Juni 2002.

752 Siehe dazu: „D. Selbstidentifikation kultureller Distinktion als Kern der Definitionsansätze“ auf S. 114. 
Voraussetzungen oder Kategorisierungen festzuschreiben. Es fehlt hingegen der Aspekt einer Selbstidentifikation oder eines Ausdrucks der Zusammengehörigkeit.

Es zeigt sich weiterhin eine Verbindung des Konzepts ,cultural community mit den Debatten zur Rolle der Nation und des Staates. So findet sich unter dem Stichwort ,nation 'im Glossar folgender Absatz:

The term 'cultural communities' is intended to be broad enough to include the nationals of an entire country, a 'nation', in cases where traditional cultural expressions are regarded as 'national folklore' and belonging to all of the people of a particular country. This complements and accords with the practice in other policy areas. ${ }^{753}$

Eine ähnliche Interpretation findet sich in der Kommentierung der Revised Objectives and Principles:

The term 'cultural communities' is intended to be broad enough to include also the nationals of an entire country, a 'nation', in cases where TCEs/EoF are regarded as 'national folklore' and belonging to all of the people of a particular country. This complements and accords with the practice in other policy areas. Therefore, a national law could, for example, state that all nationals are the beneficiaries of protection. ${ }^{754}$

Trotz dieser vielschichtigen Bedeutung des Konzepts ,cultural community; die über eine Beschreibung substaatlicher Gruppen hinausgeht, wird der Begriff nicht als Alternative für den Begriff, nation'verwendet. Vielmehr findet er in seiner Unbestimmtheit und Offenheit Zustimmung, die sowohl substaatliche Gruppen als auch die gesamte Bevölkerung eines Staates umfassen kann. ${ }^{755}$ Diese Auslegung wird unterstützt durch die Beiträge der Staaten, die sowohl den Begriff, nation' in die Entwürfe einfügen möchten, als auch den Begriff ,cultural community " unterstützen. ${ }^{756}$ Durch diese kumulative Verwendung beider Begriffe widersprechen sie implizit einer begrifflichen Alternativität beider Begriffe. Die Vermittlerin Kim Connelly-Stone vermerkt darüber hinaus, dass der Begriff ,cultural

\footnotetext{
753 WIPO IGC, „Glossary of Key Terms Related to Intellectual Property and Traditional Cultural Expressions“, WIPO/GRTKF/IC/19/INF/7, Annex, Stichwort „Nation“.

754 WIPO IGC, „The Protection of Traditional Cultural Expressions/Expressions of Folklore: Revised Objectives and Principles“, WIPO/GRTKF/IC/17/4, Annex, Art. 2, Commentary, S. 21.

755 WIPO IGC, „Nineteenth Session, July 18 to 22, 2011, Report“, WIPO/GRTKF/IC/19/12, Mexico: Abs. 80; EU: Abs. 92.

756 WIPO IGC, „Seventeenth Session, December 6 to 10, 2010, Report“, WIPO/GRTKF/IC/17/12, Abs. 66.
} 
community 'Gemeinschaften in der Diaspora beschreiben könnte und öffnet somit einen weiteren Bedeutungshorizont. ${ }^{757}$

,Cultural communities' können somit neben substaatlichen Gruppen auch die Gemeinschaft einer Nationalkultur oder Träger einer , nationalen Folklore' beschreiben und dadurch die Gruppe aller Staatsbürger umfassen. Vor dem Hintergrund der Diskussion um die Einbeziehung der ,Nation' als Berechtigte eines Instruments an TK/TCEs,${ }^{758}$ lässt sich eine weitergehende Konkretisierung dahingehend vollziehen, dass nur das Staatsvolk von Kleinst- und Inselstaaten mit einer geringen Bevölkerungsgröße als ,cultural community 'bezeichnet werden kann. Für die Anwendbarkeit neuer Rechtsinstrumente auf größere Staaten als Berechtigte zeigten sich während der Verhandlungen im IGC divergierende Meinungen.

Für eine Bestimmung des rechtlichen Verhältnisses zwischen dem neuen Konzept ,cultural community ' und den bestehenden Konzepten substaatlicher Gruppen ergibt sich aus der Unbestimmtheit des neuen Begriffs eine Vielzahl verschiedener Auslegungsmöglichkeiten. ${ }^{759}$ Versteht man den Begriff ,cultural community ' in seiner offenen Bedeutung im Sinne des IGC-Glossars, so würden alle bereits bestehenden substaatlichen Gruppen auch als ,cultural community' anerkannt werden können. Die Beschreibung dieses Konzept als „,social unit whose members experience strong feelings of unity and solidarity and which is distinguished from other communities by its own culture or cultural design, or by a variant of the generic culture. “760 ist so offen gestaltet, dass Gruppen von Minderheiten, indigenen Völkern sowie lokalen Gemeinschaften ebenfalls als ,cultural community' anerkannt und entsprechende Rechte an TK/TCE beanspruchen könnten. Die Definition von Gruppen über das Gefühl der Zusammengehörigkeit und eine kulturelle Distinktion wird auch vom Kern aller Definitionsansätze der etablierten Konzepte substaatlicher Gruppen umfasst, ${ }^{761}$ so dass sich eine überlagernde Anwendbarkeit mit den bestehenden Konzepten substaatlicher Gruppen rechtlich vertreten lässt. Ebenso erscheint es in Anbetracht dieses offenen Konzepts als möglich, dass neue Gruppen im Zuge der Entstehung kollektiver Identität als ,cultural community ‘ anerkannt werden.

Sollte sich eine Interpretation des neuen Konzepts, cultural community' im Sinne einer zwingenden Parallelität zur Nation oder zum gesamten Staatsvolk etablieren,

${ }^{757}$ WIPO IGC, „Nineteenth Session, July 18 to 22, 2011, Report“, WIPO/GRTKF/IC/19/12, Annex II, S. 3.

${ }^{758}$ Siehe dazu „B. III. Diskussionen um die Berechtigung der ,Nation“ und die Rolle des Staates“ auf S. 218.

${ }^{759} \mathrm{Zu}$ den Hintergründen der Analyse des rechtlichen Verhältnisses der unterschiedlichen Konzepte zueinander siehe: „E. Das rechtliche Verhältnis der Konzepte zueinander“ auf S. 116.

760 WIPO IGC, „Glossar“, WIPO/GRTKF/IC/20/INF/13, Annex, Stichwort „Cultural Community".

${ }^{761}$ Siehe dazu: „D. Selbstidentifikation kultureller Distinktion als Kern der Definitionsansätze“ auf S. 114. 
ergäbe sich eine andere rechtliche Bewertung. Minderheiten, dies bestimmt bereits die Begrifflichkeit, könnten in ihrer numerischen Unterlegenheit nicht die Gruppe eines gesamten Staatsvolks ausmachen und somit auch nicht als ,cultural community" in diesem Sinne verstanden werden. ${ }^{762}$ Ähnliches ergäbe sich für lokale Gemeinschaften. Zwar lässt die gleichlautende Bezeichnung als ,community" eine überlagernde Anwendung möglich erscheinen. Eine Interpretation der Bedeutung , cultural community" als vergleichbar mit dem über eine Nationalkultur geeinten Staat widerspräche den Implikationen der Bezeichnung , local community', die sich gerade durch ihre lokale Verwurzelung im Vergleich zu anderen Gruppen im Staat auszeichnet. In Staaten, in denen sich das Staatsvolk aus indigenen Völkern konstituiert, wäre ein Gleichlauf zur Anwendbarkeit des Begriffs ,cultural community" vertretbar. Auch wenn der Begriff, indigenes Volk' keine Aussage über ihre Rolle im Verhältnis zum Staatsvolk aussagt, finden sich wenige Staaten, in denen indigene Völker im Sinne eines Staatsvolks unter die Bezeichnung einer ,cultural community fallen würden.

\section{Zusammenfassung}

Die Debatten um die Beschreibung der beneficiaries eines Rechts an TK/TCEs im $I G C$ beziehen sich vorrangig auf bereits in anderen internationalen Instrumenten verwendete Konzepte substaatlicher Gruppen und befassen sich nur bedingt mit Definitionsansätzen dieser Begrifflichkeiten. Es geht weniger um ein Aushandeln konkreter Definitionen als vielmehr um ein Verhandeln mit komplexen Rechtsbegriffen, deren Bedeutungen in anderen Bereichen nähere Bestimmung erfahren haben und weiterhin einem konstanten Diskurs der Konkretisierung unterliegen. Im IGC wurden bisher unterschiedliche Ansätze zur Regelung der beneficiaries verhandelt, die von einzelnen Individuen über verschiedene Begriffe, die eine Gruppe beschreiben, bis hin zum Staat reichen. Im Zentrum der Debatten stehen insbesondere die Konzepte, indigenous Peoples;, indigenous and local communities', ,cultural communities', minorities' und ,nations'sowie Individuen und Familien.

Die Debatten um Definitionen der neuen Begrifflichkeiten der ,traditional communities' und ,cultural communities" sind nur bedingt vorangeschritten, sodass in den Kommentaren zu den Entwürfen der Hinweis auf eine noch ausstehende, tiefer gehende Klärung gemacht wird. ${ }^{763}$ In den draft articles zu TCEs bezieht sich Artikel

\footnotetext{
762 Aussagen der chinesischen Delegation machten jedoch deutlich, dass ein Gruppenrecht an GR/TK/TCE auch auf Minderheiten Anwendung finden soll.

763 WIPO Generalversammlung, „Matters Concerning the Intergovernmental Committee on Intellectual Property and Genetic Resources, Traditional Knowledge and Folklore (IGC)“, $\mathrm{WO} / \mathrm{GA} / 40 / 7$, Annex A, S. 8.
} 
2 nur noch auf die bereits etablierten Konzepte zur Bestimmung der beneficiaries. ${ }^{764}$ Weiterhin wird deutlich, dass die asiatischen, afrikanischen sowie die Kleinst- und Inselstaaten auf eine Begrifflichkeit drängen, die in ihrer Interpretation die Gruppe aller Staatsangehörigen als beneficiaries einschließt. Die Verhandlungen drehen sich dabei insbesondere um die Verwendung des Begriffs ,nation' oder eine entsprechende Interpretation des neuen Konzepts ,cultural community: Ein Konsens zeichnet sich dahingehend ab, dass die Kleinst- und Inselstaaten jeweils mit ihrem gesamten Staatsvolk als Berechtigte neuer Instrumente für TK/TCEs agieren dürfen. Entgegen den Forderungen afrikanischer und asiatischer Staaten zeichnet sich für diese in Anbetracht ihrer durch die koloniale Grenzziehung zumeist multiethnische und -kulturelle Bevölkerungszusammensetzung keine entsprechende $\mathrm{Zu}$ stimmung ab.

Eine rechtliche Bewertung der kumulativen Verwendung der unterschiedlichen Konzepte, die sich gegenwärtig in den Verhandlungen des IGC abzeichnet, spricht dafür, dass neue Instrumente an TK/TCEs globale Verbreitung finden können. Im Kontext der globalen Indigenenbewegung und auch im internationalen Umweltrecht haben sich indigene Völker und lokale Gemeinschaften insoweit etabliert, als dass diese Gruppen entsprechende Rechte geltend machen werden können.

${ }^{764}$ WIPO Generalversammlung, „Matters Concerning the Intergovernmental Committee on Intellectual Property and Genetic Resources, Traditional Knowledge and Folklore (IGC)“, WO/GA/41/15, Annex C, S. 7. 


\section{Die Anerkennung von Berechtigten: die NCA und neue Akteursverhältnisse}

Die Verhandlungen zur Beschreibung und Bestimmung der beneficiaries der neuen Rechtsinstrumente zum Schutz von TK/TCEs konnten, wie auch die Definitionendebatten der bereits etablierten Konzepte substaatlicher Gruppen, keinen Konsens einer juristischen Definitionen für die Rechtsträger finden. Mangels einer subsumierbaren, abstrakt-generellen Definition ergibt sich somit hier eine vergleichbare Problemlage wie bei den etablierten Konzepten substaatlicher Gruppen. Diese werden hier meist sogar selbst als Rechtsträger in den aktuellen Entwürfen verhandelt. Die neuen Gruppenrechtsinstrumente zum Schutz und zur Förderung traditionellen Wissens und traditioneller kultureller Ausdrucksformen beziehen sich ebenso wie die bekannten drei Konzepte substaatlicher Gruppen auf die kulturelle Identität einer Gruppe. Daher lässt sich die in Teil 3 vorgestellte Perspektive der konstitutiven Anerkennung zur Bestimmung von substaatlichen Gruppen auch auf den Kontext der im IGC verhandelten Rechtsinstrumente übertragen.

Eine entscheidende Rolle kann in diesem Zusammenhang der in den Regelungen des management of rights angedachten national competent authority zukommen. ${ }^{765}$ Eine solche Institution soll jeweils als staatliche oder zwischenstaatliche Behörde geschaffen werden, um Ansprüche und Rechte der berechtigten Gruppen an GR/TK/TCEs gegenüber etwaigen Nutzern im Auftrag der Berechtigten zu vertreten. ${ }^{766}$ Die NCA würde sich als eine zentrale Institution und Bezugspunkt für substaatliche Gruppen in Rechtsfragen zum Schutz von TK/TCEs etablieren, der gleichzeitig eine entscheidende Rolle bei Fragen der Anerkennung zukommen würde. Als zentraler Akteur würden hier die innerstaatlich bisher zumeist nicht geregelten Fragen der Anerkennung zusammenlaufen können. Die bisherigen Formulierungen der Entwürfe, dass also die NCA ,auf Anfrage und im Auftrag der Berechtigten“ und erst durch eine gegebene „Ermächtigung zum Handeln“ aktiv werden solle, bringen diese neue Institution in eine Schlüsselposition des Dialogs der Anerkennung kollektiver Identitäten. Übernimmt die NCA die Verwaltung der Rechte einer Gruppe, erkennt sie diese als etablierte substaatliche Gruppen im Sinne der neuen Instrumente der Rechte an TK/TCEs an. Als staatliche Institution würde dies die Anerkennung seitens des Heimatstaates implizieren und den Rechtsstatus substaatlicher Gruppen national und international garantieren.

\footnotetext{
765 WIPO IGC, „The Protection of Traditional Cultural Expressions: Draft Articles“,

WIPO/GRTKF/IC/22/4, 27. April 2012, Art. 4; WIPO IGC, „The Protection of Traditional Knowledge: Draft Articles“, WIPO/GRTKF/IC/21/4, Art. 5.

766 Siehe dazu „A. VII. Durchsetzungsmechanismen“, auf S. 207.
} 
Mit der Einrichtung einer NCA würde sich potentiell ein Dreiecksverhältnis institutionalisieren und somit eine neue Akteurskonstellation zwischen berechtigter Gruppe, Staat und Nutzer schaffen. Als urheberrechtsähnliches Instrument geistigen Eigentums etablierten die neuen Rechte an TK/TCEs - anders als die völkerrechtlichen Instrumente $\mathrm{zu}$ den bereits bestehenden Konzepten - kein rechtliches Verhältnis zwischen der Gruppe und ihrer Staatsregierung. Vielmehr konstituierten neue Rechte an TK/TCEs die Grundlage möglicher Beziehungen zwischen den berechtigten Gruppen unmittelbar gegenüber Dritten, also Privaten oder öffentlich-rechtlichen Akteuren als Nutzer von TK/TCEs in Drittstaaten. Es stünden somit die Heimatstaaten der Gruppen in Form der NCA an der Seite der Berechtigten, um gemeinsam die Rechte an TK/TCEs gegenüber in- und ausländischen Nutzern zu vertreten. Vor dem geopolitischen Hintergrund der Konfliktsituationen in diesem Kontext ergäbe sich eine Stärkung substaatlicher Gruppen aus dem globalen Süden in Form einer Unterstützung durch ihre Heimatstaaten gegenüber den Nutzern im, Westen'.

Des Weiteren veränderte sich durch die Schaffung neuer Rechte an TK/TCEs für substaatliche Gruppen das sonst bipolare Verhältnis zwischen indigenen Völkern und lokalen Gemeinschaften gegenüber ihren Heimatstaaten. Die Staaten hätten ein Interesse daran, substaatliche Gruppen als Berechtigte TKs/TCEs in ihrem Staat anzuerkennen, um gemeinsam mit diesen Gruppen Ansprüche gegenüber Nutzern in Drittstaaten geltend machen zu können. Die bestehenden völkerrechtlichen Instrumente bezüglich substaatlicher Gruppen normieren bisher ausschließlich staatliche Verpflichtungen gegenüber Minderheiten, indigenen Völkern oder lokalen Gemeinschaften, seien dies Regelungen zum Minderheitenschutz, zur Gewährung etwaiger Sonderrechte, zur Übertragung von Hoheitsrechten, der Verpflichtungen zur Beteiligung bei umweltrelevanten Entscheidungen oder die Einhaltung erhöhter Beteiligungsrechte des prior informed consent bei der Freigabe genetischer Ressourcen. Anders als bei den bestehenden Regelungen für substaatliche Kollektive, wo die Staaten gegenüber diesen Gruppen unterschiedliche Verpflichtungen eingehen, wenn sie Gruppen als ,Minderheit', ,indigenes Volk ${ }^{\star}$ oder ,lokale Gemeinschaft ${ }^{`}$ anerkennen, würde die Anerkennung substaatlicher Gruppen als Berechtigte an TK/TCEs nun vorrangig Rechte gegenüber Nutzern in Drittstaaten schaffen. Die neuen Regelungen zum Schutz von TK/TCEs bewirken somit ein grundsätzlich neues Akteursverhältnis und eine Konstellation von politischen Interessen, bei der die Anerkennung substaatlicher Gruppen als Berechtigte für die Regierungen von Vorteil wäre.

Gleichzeitig konstituierte die angedachte Schaffung einer NCA als Regierungsbehörde eine Konzentration staatlicher Macht in Bezug auf die Anerkennungskompetenz und die Rechtsausübung der Rechte an TK/TCEs gegenüber substaatlichen Gruppen. Prozesse der Anerkennung würden somit zunehmend verrechtlicht und wären nicht mehr den jeweiligen politischen Gegebenheiten ausgeliefert an denen bislang eine Vielzahl nationaler und internationaler staatli- 
cher und nicht-staatlicher Akteure beteiligt sind. Durch die Zentralisierung des management of rights für TK/TCEs würden substaatliche Gruppen allerdings in ihrer Rechtsausübung unter die Kontrolle des Staates gestellt werden. Bei widerstreitenden Interessen zwischen Gruppe und Staat wäre Letzterem durch die NCA ein unmittelbarer Einfluss auf die konkrete Rechtsausübung garantiert und er könnte als Bevollmächtigter gegenüber Nutzern im Ausland auch im Sinne eigener Interessen handeln. 


\section{Zusammenfassung}

Die Verhandlungen des WIPO IGC zu neuen Schutzinstrumenten geistigen Eigentums und GR/TK/TCEs verknüpfen die Themenkomplexe der Regelungen zu GR und assoziiertem TK auf der einen Seite und TCEs und TK im weiteren Sinne auf der anderen. Während Fragen zu GR in ihrer jüngeren Geschichte bisher hauptsächlich im Kontext des internationalen Umweltrechts verhandelt wurden, setzen die Verhandlungen zu TK/TCEs eine über 30-jährige Geschichte der Bestrebungen zur Einführung von Schutzinstrumenten geistigen Eigentums für kollektiv-generierte kulturelle Ausdrucksformen fort. Eines der Kernprobleme der Verhandlungen im IGC ist die rechtliche Bestimmung der Berechtigten möglicher neuer Instrumente an TK/TCEs, um sicherzustellen, dass die eigentlichen Träger des Wissens und der Kultur als beneficiaries normiert werden.

Die Analyse des Verhandlungsgegenstands der beneficiaries des WIPO IGCs hat gezeigt, dass unter den gegenwärtig verwendeten Begriffen für neue Instrumente zum Schutz von TK/TCEs hauptsächlich an bestehende Konzepte substaatlicher Gruppen angeknüpft wird und nur teilweise neue Begrifflichkeiten Anwendung finden. Es sollen indigenous peoples/communities und local communities als Berechtigte ihres traditionellen Wissens und ihrer traditionellen kulturellen Ausdrucksformen fungieren. Gleichzeitig soll eine national competent authority die Rechteausübung übernehmen können, die als zentrale Regierungsinstitution eine entscheidende Rolle im konstitutiven Prozess der Anerkennung von Gruppen als Berechtigte erlangen würde.

Die neuen Instrumente geistigen Eigentums an TK/TCEs würden ein urheberrechtsähnliches Verhältnis zwischen Berechtigten und Nutzern schaffen, welches zusammen mit der NCA ein neues Drei-Akteurs-Verhältnis bilden würde. In diesem Gefüge wäre es - anders als bei den bestehenden Instrumenten zum Schutz substaatlicher Gruppen - im Interesse des Staates, Gruppen als Berechtigte und Träger von TK/TCEs anzuerkennen. Gleichzeitig wird die eigentliche Ausübung der Rechte der Gruppen durch die NCA institutionell an den Staat gebunden, was seine Rolle bei möglichen Interessenkonflikten gegenüber den substaatlichen Gruppen oder Nutzern im Ausland stärkt. Vor dem Hintergrund der rechtlich möglichen überlagernden Anwendbarkeit der unterschiedlichen Konzepte substaatlicher Gruppen könnte die Anerkennung von Gruppen im Kontext der Rechte an TK/TCE Einfluss auf die Anerkennung der Gruppen in anderen Kontexten nehmen. Insofern könnte die zunehmende Konstituierung und Anerkennung substaatlicher, kollektiver Identitäten als etablierte Gruppen im Kontext der Rechte an TK/TCE auch die Anerkennung dieser Gruppen als ,Minderheiten', indigene Völker' oder ,lokale Gemeinschaften' positiv beeinflussen. 


\section{Ergebnis}

Angesichts der kulturellen Heterogenität der Weltbevölkerung bedarf es Regelungen, welche die Beziehungen zwischen unterschiedlichen Gruppen regeln. Zusätzlich zur internationalen Ordnung der Staaten entwickelten sich dazu im Völkerrecht für substaatliche Gruppen drei verschiedene Konzepte: ,Minderheiten', indigene Völker' und ,lokale Gemeinschaften'. Problematisch ist dabei seit jeher die Formulierung von Definitionen, die in rechtlicher Art und Weise beschreiben, welche Gruppen mit dem jeweiligen völkerrechtlichen Konzept gemeint sein sollen. Bisher konnte keiner der Vorschläge einer Definition allgemeine Zustimmung finden.

Die hier vorgelegte Analyse der drei Konzepte hat gezeigt, dass alle bisherigen Definitionsansätze substaatlicher Gruppen im Kern auf eine ,Selbstidentifikation kultureller Distinktion' der Gruppe rekurrieren: Demnach wird als Ausgangspunkt immer eine von der Gruppe geäußerte Identifizierung ihrer eigenen, von der Mehrheit unterscheidbaren Kultur angenommen. Aus einer rechtlichsystematischen Perspektive ergibt sich in Anbetracht dieses gemeinsamen Kerns in allen drei Konzepten, dass sie überlagernd auf dieselbe Gruppe anwendbar sein könnten, sofern diese die jeweils zusätzlich notwendigen Merkmale aufweisen. Exemplarisch lässt sich dies folgendermaßen skizzieren: Indigene Völker und lokale Gemeinschaften können ,Minderheiten' sein, sofern sie sich im Verhältnis zur Mehrheitsgesellschaft in der Unterzahl befinden. Minderheiten und lokale 
Gemeinschaft könnten Ansprüche auf Selbstbestimmung als ,indigene Völker geltend machen, sofern sie Verbindungen zu präkolonialen Gesellschaften aufweisen oder ihre gegenwärtige politische Position mit der Marginalisierung kolonisierter Völker vergleichbar ist. Minderheiten und indigene Völker könnten als, lokale Gemeinschaften' im Sinne des Umweltvölkerrechts gelten, sofern sie subsistenzwirtschaftlich und biodiversitätsfördernd leben.

Der Blick auf die Praxis zeigt nun, dass mangels juristischer Definitionen substaatliche Gruppen nach einer Selbstidentifikation als ,Minderheit', indigenes Volk' oder ,lokale Gemeinschaft ${ }^{`}$ entsprechend im Sinne eines der völkerrechtlichen Konzepte anerkannt werden. Diese Anerkennung wurde in Teil 3 der vorliegenden Arbeit als neue Perspektive zum Verständnis der Anwendung der Konzepte substaatlicher Gruppen vorgestellt. Im Vergleich zur Staatenanerkennung kommt der Anerkennung substaatlicher Gruppen jedoch konstitutive Wirkung zu. Sie bildet das Pendant zur vorausgehenden Selbstidentifikation im Sinne eines der Konzepte. Für eine rechtliche Beschreibung der Anwendung der Konzepte muss folglich nicht mehr auf die bisher diskutierten Definitionsansätze zurückgegriffen werden. Aus der Perspektive der Anerkennung wird deutlich, dass trotz der Vielzahl von beteiligten Akteuren letztlich die Heimatstaaten die Macht der Anerkennung inne haben, was die den Konzepten substaatlicher Gruppen zugrunde liegende Idee - insbesondere ihre Funktion als Schutzinstrument gegenüber der Regierung des Heimatstaates - ad absurdum führt.

Betrachtet man die Prozesse der Anerkennung aus interdisziplinärer Perspektive, so zeigt sich, dass das Völkerrecht mit seinen drei Konzepten substaatlicher Gruppen nicht die notwendige Flexibilität aufbringt, um der vorfindlichen kulturellen Vielfalt kollektiver Identitäten und ihrem stetigen Wandel gerecht zu werden. Um kulturellen Praxen einen gruppenrechtlichen Schutz gewähren zu können, müssen sich Gruppen mit ihrer kollektiven Identität an den holistischen Vorstellungen eines der Konzepte messen lassen, um gegebenenfalls als ,Minderheit', ,indigenes Volk' oder , lokale Gemeinschaft' anerkannt zu werden. Angesichts der dabei wirkenden Zuschreibungen unterliegen die Gruppen einem einseitigen Prozess, verkennender Anerkennung, auf den sie im Rahmen der gegenwärtigen völkerrechtlichen Strukturen nur unzureichend Einfluss nehmen können. Insbesondere mangelt es unter den bestehenden Regeln an effektiven Formen, die Gruppen am Prozess der Aushandlungen zu beteiligen. Dies gilt sowohl bezüglich der Gestaltung der normativen Konzepte, als auch der eigentlichen Anerkennung substaatlicher kollektiver Identitäten als ,Minderheit', indigenes Volk ${ }^{6}$ oder , lokale Gemeinschaft'. Zusammenfassend legen die interdisziplinären Einsichten nahe, dass die völkerrechtlichen Konzepte de lege ferenda eine differenzierte Anwendung bezüglich einzelner Praxen ermöglichen sowie substaatliche Gruppen im Prozess der Gestaltung und ihrer Anerkennung unter den Konzepten beteiligen sollten. Diesen Anforderungen müsste auch eine dazu eingesetzte internationale Institution genügen. 
Die aktuellen Diskussionen zu völkerrechtlichen Gruppenrechten finden in den gegenwärtigen Verhandlungen des WIPO IGC statt. Im Zuge der Entwicklung neuer gruppenrechtlicher Instrumente zum Schutz von TK und TCEs wird dabei vornehmlich auf die bestehenden Konzepte der ,indigenous peoples/communities ‘ und ,local communities' zurückgegriffen. Es ist angedacht, dass eine national competent authority substaatliche Gruppen bei der Geltendmachung ihrer Rechte unterstützt. Es entstünde dadurch eine erweiterte Akteurskonstellation, in welcher der Staat gemeinsam mit seinen substaatlichen Gruppen gegenüber potentiellen Nutzern auftreten würde. Im Vergleich zur strukturellen Gestaltung aller bisherigen Konzepte für substaatliche Gruppen - in denen der Staat Sonderrechte für Minderheiten gewähren, Kompetenzen an indigene Völker übertragen oder Zustimmung lokaler Gemeinschaften zum bioprospecting erhalten muss - läge somit eine Anerkennung kollektiver Identitäten im Interesse der Staaten.

Auch in Anbetracht der aktuellen Entwicklungen im WIPO IGC gilt es, nicht bei den kriterialen Definitionsansätzen substaatlicher Gruppen stehen zu bleiben, sondern den Prozess der konstitutiven Anerkennung in den Blick zu nehmen. Nur so können die hintergründigen Machtstrukturen offen gelegt, die notwendigen Voraussetzungen für einen angemessenen Anerkennungsprozess erarbeitet und eine individuelle Aushandlung gegenseitiger Anerkennung kultureller Identitäten auf Augenhöhe möglich werden. 



\section{Zusammenfassung in Thesen}

Die Instrumente des internationalen Minderheitenschutzes sollen die kulturelle Integrität von Minderheitengruppen bewahren und diese vor kultureller Assimilation durch die Mehrheitsgesellschaft schützen. Unter dem Begriff der ,Minderheit' wird eine im Verhältnis zur Mehrheitsgesellschaft eines Staates numerisch kleinere und ethnisch, sprachlich, religiös oder anderweitig kulturell distinkte Gruppe verstanden, die diese Distinktion durch einen ,Ausdruck des Gefühls der Zusammengehörigkeit' deutlich macht. Einige internationalrechtliche Instrumente in Europa formulieren zusätzlich die Voraussetzungen der Staatsangehörigkeit des Heimatstaates sowie eine historisch-geographische Verwurzelung in der Region, in der sie lebt.

Vertreter indigener Gruppen artikulieren seit den 1970er Jahren auf internationaler Ebene einen umfassenden Anspruch auf Selbstbestimmung, der in noch umfassenderer Weise einer kulturellen Assimilation entgegen wirken soll. Der Anspruch indigener Völker auf Selbstbestimmung wird mittlerweile nicht mehr im Sinne einer eigenen Staatswerdung, sondern im Kontext der Menschenrechte interpretiert und umfasst eine weitgehende Autonomie indigener Gruppen im Rahmen der Grenzen ihrer Heimatstaaten. Als ,indigene Völker' werden kulturell distinkte Gruppen mit einer historischen Verbindung zu präkolonialen Gesellschaften verstanden, die sich über eine gemeinsame Zusammengehörigkeit 
identifizieren und ihre Kultur zukünftigen Generationen weitergeben möchten. Die Selbstidentifikation als ,indigen' soll dabei der Ausgangspunkt einer Anwendung des Indigenitätskonzepts sein. Einige Interpretationen des Begriffs ,indigene Völker' rekurrieren speziell auf eine Lebenssituation der Marginalisierung und Unterdrückung durch eine dominierende Mehrheitsgesellschaft. Der historische Kontext der Kolonisierung wird dabei in den Hintergrund geschoben. In Form dieser Uminterpretation des Begriffs werden Ansprüche indigener Gruppen auch in Asien und Afrika artikuliert, wo das historische Differenzierungsmoment der Verbindung zu präkolonialen Gesellschaften keine tragfähigen Ergebnisse liefert.

Das Konzept der ,lokalen Gemeinschaft ${ }^{`}$ findet sich im internationalen Umweltrecht und bezeichnet substaatliche Gruppen, welche durch die Nutzung ihres traditionellen Wissens in nachhaltiger Art und Weise, meist in entlegenen Gebieten, subsistenzwirtschaftlich leben. Es fußt auf den internationalrechtlichen Entwicklungen der farmer's rights und indigener Völker. Die umweltvölkerrechtlichen Instrumente schreiben ,lokalen Gemeinschaften' eine Abhängigkeit von ihrer Umwelt sowie eine nachhaltige Lebensweise in enger Verbindung mit ihrem Ökosystem zu und erkennen gleichzeitig ihren entscheidenden Beitrag zum Schutz biologischer Vielfalt an. Insbesondere die CBD garantiert diesen Gruppen unter dem Begriffspaar der, indigenen und lokalen Gemeinschaft ${ }^{`}$ ein System des access and benefit sharing mit Zustimmungsvorbehalten eines prior informed consent zum bioprospecting, welches ihre genetischen Ressourcen und ihr assoziiertes traditionelles Wissen umfasst.

Für die drei völkerrechtlichen Konzepte substaatlicher Gruppen rekurrieren die jeweiligen Ansätze von rechtlichen Definitionen der Gruppen in ihrem Kern auf die Selbstidentifikation kultureller Distinktion. Ian Brownlie formulierte diese These, indem er sagte:

The heterogeneous terminology which has been used over the years - the references to nationalities, peoples, minorities and indigenous peoples - involves essentially the same idea. ${ }^{767}$

Zusätzlich zu dieser, selben Idee, die den Kern jeder der drei Konzepte substaatlicher Gruppen ausmachen soll, zeigen sich historisch gewachsene Prägungen der unterschiedlichen Konzepte. Dies sind insbesondere die Merkmale der numerischen Minderzahl gegenüber der Mehrheit, die Staatsbürgerschaft des Heimatstaats, die Verbindung zu präkolonialen Gesellschaften oder eine histori-

\footnotetext{
${ }^{767}$ Brownlie, „The Rights of Peoples in Modern International Law“, S. 5.
} 
sche und gegenwärtige Marginalisierung sowie die biodiversitätsfördernde, subsistenzwirtschaftliche Lebensweise.

Die Konzepte sind kumulativ auf dieselbe Gruppe anwendbar, soweit die Merkmale der anderen positivrechtlich verankerten Definitionen gegeben sind. So können Gruppen von indigenen Völkern oder lokalen Gemeinschaften gleichzeitig ,Minderheiten' sein, sofern sie in ihrem Heimatstaat numerisch in der Minderzahl sind. Sollten Minderheiten oder Indigene in ihrer kulturellen Distinktion traditionelles Wissen in Bezug zu einer nachhaltigen subsistenzwirtschaftlichen Lebensweise aufweisen, fielen sie unter die Bezeichnung der ,indigenen und lokalen Gemeinschaft'. Nach den Anfängen der internationalen Indigenenbewegung in den 1970er Jahren in den typischen Siedlerstaaten zeigen die aktuellen Entwicklungen, dass lokale Gemeinschaften und Minderheiten auch in Asien und Afrika aufgrund ihrer Marginalisierung und Unterdrückung als ,indigene Gemeinschaften`anerkannt werden.

Mangels allgemein konsentierter, abstrakt-genereller Definitionen werden die Gruppen entsprechend ihrer Selbstidentifikation als ,Minderheit', indigenes Volk' oder ,lokale Gemeinschaft ${ }^{6}$ anerkannt. Die Anerkennung substaatlicher Gruppen wird hier als neue Perspektive eines rechtlichen Verständnisses der Anwendung der Konzepte substaatlicher Gruppen vorgeschlagen. Anerkennung ist für den rechtlichen Status der Gruppen konstitutiv, denn anders als bei der Staatenanerkennung stehen substaatliche Gruppen in einem Über/Unterordnungsverhältnis zu ihren Heimatstaaten. Die Anerkennung bildet das Pendant zur Selbstidentifikation substaatlicher Gruppen. Die Anerkennung substaatlicher Gruppen ist völkerrechtlich nicht geregelt und wird national auf unterschiedlichste Weise ausgestaltet. Die beispielhaft dargestellten Anerkennungsverfahren offenbaren eine Bandbreite von impliziten Anerkennungen im Politischen bis hin zu expliziten Regelungen, die der Anerkennung einen institutionalisierten Rahmen geben und konkrete Vorgaben machen. Neben den Heimatstaaten der Gruppen spielen dabei Drittstaaten, internationale Organisationen, Nichtregierungsorganisationen oder private Akteure eine Rolle. Die Perspektive der Anerkennung öffnet den Blick auf die Machtstrukturen im Prozess der Anwendung der Konzepte substaatlicher Gruppen. Neben der Perspektive der konstitutiven Anerkennung werden die Definitionsdebatten zur Bestimmung des Rechtsstatus' substaatlicher Gruppen entbehrlich.

Definitionsansätze und Anerkennung kollektiver Identitäten sind ebenfalls Gegenstand der kulturwissenschaftlichen Forschung und der politischen Theorie, die sich mit kulturell distinkten Gruppen in der sozialen Wirklichkeit befassen. In der Kulturwissenschaft werden Minderheiten und indigene Völker nicht mehr mit essentialisierenden, kriterialen Definitionen erfasst, wie dies positivistische 
Definitionsansätze versuchen. Vielmehr werden kulturell distinkte Gruppen in ihrer Identifizierung im Prozess der situativen Aushandlung ihrer kollektiven Identität verstanden. In der politischen Theorie impliziert die Anerkennung substaatlicher Gruppen immer auch die Zuschreibung konkreter Eigenschaften von Seiten der dominierenden Mehrheitsgesellschaft. Dabei können substaatliche Gruppen durch die Projektionen, welche die Mehrheit in ihrem Bild von ,den Anderen“ auf diese zurückspiegelt, Schaden nehmen, wenn sie Objekt verkennender und herabwürdigender Zuschreibungen sind.

Das Völkerrecht verankert die Bilder von ,den Anderen“ in Form der drei positivrechtlich normierten Konzepte substaatlicher Gruppen und konstituiert darin starre holistische Vorstellungen kollektiver Identitäten, die der sozialen Wirklichkeit nicht gerecht werden. Die Bestimmung substaatlicher Gruppen sollte daher im Sinne der hier vorgeschlagenen Perspektive der Anerkennung über Prozesse und Institutionen und nicht über kriteriale Definitionen geregelt werden.

Der gegenwärtig mangelnde völkerrechtliche Rahmen für Anerkennungsprozesse eröffnet den Heimatstaaten einen erheblichen Entscheidungsspielraum und reproduziert so das Machtgefüge, gegen welches die Gruppenrechte eigentlich einen Ausgleich schaffen sollen. Für einen Anerkennungsprozess zum Schutz substaatlicher Gruppen bedarf es de lege ferenda Institutionen, in denen Gruppen in einem transparenten Verfahren die Fragen der Anerkennung aushandeln können und in dem letztlich eine von den beteiligten Parteien unabhängige Instanz über den Rechtsstatus der Gruppe entscheidet. Für eine individuelle Ausgestaltung von Gruppenrechtsinstrumenten sind de lege ferenda differenzierbare Konzepte notwendig, welche eine auf konkrete Praxen bezogene, kollektive Identität berücksichtigen könnten, um so die verkennenden und deformierenden Einflüsse im Prozess der Aushandlung vermeiden zu können. Die Aushandlung von Konzepten und die Anerkennung einzelner Gruppen bedürften der Beteiligung sowohl der substaatlichen Gruppen als auch der Mehrheit, um den Grad der Verkennung zu reduzieren; eine Vorgabe, der auch eine internationale Institution der Anerkennung genügen müsste.

Im WIPO IGC zu GR/TK/TCEs werden die Themenbereiche GR und assoziiertes $T K$ des internationalen Umweltrechts mit den jahrzehntelangen Debatten zum Schutz von TCEs und TK im weiteren Sinne verknüpft. Dabei stehen die asiatische und afrikanische Gruppe sowie die Formation der like-minded states mit ihrer Forderung nach neuen kollektivrechtlichen Schutzinstrumenten für TK/TCEs den westlichen Staaten der Gruppe B gegenüber, welche die Verhandlungen seit Beginn verzögern. Zur Normierung der beneficiaries dieser neuen Rechte geistigen Eigentums werden neben der Entwicklung der neuen Begriffe 
,cultural community 'und ,traditional community 'vorrangig die bestehenden Konzepte ,indigenous peoples/communities' und ,local communities'verhandelt. Zudem wird streitig über die Einbeziehung von Nationen in den Kreis der Berechtigten diskutiert, wohingehend sich ein Kompromiss für Kleinst- und Inselstaaten abzeichnet, die in Form ihrer Staatsvölker als beneficiaries Anerkennung finden würden. Juristisch anwendbare Definitionen der neuen und bestehenden Konzepte substaatlicher Gruppen werden hingegen nicht diskutiert, sodass es zur Umsetzung neuer kollektivrechtlicher Instrumente geistigen Eigentums ebenfalls einer Anerkennung von Gruppen als beneficiaries bedarf.

Für die Praxis der Anerkennung zeichnet sich mit der Schaffung einer national competent authority, wie sie gegenwärtig vom IGC angedacht wird, ein neuer institutioneller Rahmen ab. Eine Regierungsbehörde des Heimatstaates der Gruppe würde demnach Ansprüche der beneficiaries gegenüber potentiellen Nutzern, die sich zumeist im ,westlichen' Ausland befinden werden, geltend machen dürfen. Dies würde ein neues Beziehungsgefüge zwischen den beteiligten Akteuren konstituieren: Der Staat würde in Form der national competent authority nicht mehr der Gruppe gegenüber stehen, wie dies bei allen bisherigen Rechten substaatlicher Gruppen der Fall ist, sondern mit ihr zusammen gegenüber Dritten auftreten. 



\section{Bibliographie}

\section{A. Primärquellen: Verträge, Gesetzgebung, Sitzungsunterlagen, Dokumente, Berichte, Entscheidungen, Nachrichten}

Ad hoc Expert Group Meeting of Local Community Representatives, „Identification of Common Characteristics of Local Communities“, UNEP/CBD/AHEG/LCR/INF/1, 27. Juni 2011.

Ad hoc open ended working group on Article 8(j) and Related Provisions of the Convention on Biological Diversity. „Composite report on the status and trends regarding the knowledge, innovations and practices of indigenous and local communities - Regional report: North America“, $\mathrm{UNEP} / \mathrm{CBD} / \mathrm{WG} 8 \mathrm{~J} / 3 / \mathrm{INF} / 8,7$. Oktober 2003.

-. „Composite report on the status and trends regarding the knowledge, innovations and practices of indigenous and local communities - Regional Report: Central America“, UNEP/CBD/WG8J/3/INF/6, 30. September 2003.

. „Participatory Mechanisms for Indigenous and Local Communities in the Work of the Convention“, UNEP/CBD/WG8J/7/9, 8. Juli 2011. 
- „Report of the Expert Group Meeting of Local Community Representatives within the Context of Article 8(j) and Related Provisions of the Convention on Biological Diversity“, UNEP/CBD/WG8J/7/8/ADD1, 4. September 2011.

Advisory Committee on the Framework Convention for the Protection of $\mathrm{Na}$ tional Minorities. „Opinion on Germany“, ACFC/INF/OP/I (2002) 008, 2002, 1. März 2002.

—. „Opinion on Finland“, ACFC/INF/OP/I (2001) 002, 22. September 2000.

. „Dritte Stellungnahme zu Deutschland Verabschiedet am 27. Mai 2010 sowie Stellungnahme Deutschlands zum Dritten Bericht des Beratenden Ausschusses für das Rahmenübereinkommen zum Schutz nationaler Minderheiten (2. Dezember 2010)“, ACFC/OP/III (2010) 003, 27. Mai 2010 .

African Commission on Human and People's Rights, International Work Group for Indigenous Affairs. Indigenous Peoples in Africa: The forgotten Peoples?, 2006.

- Advisory Opinion of the African Commission on Human and Peoples' Rights on the United Nations Declaration on the Rights of Indigenous Peoples, 2010, angenommen im Rahmen der 41st ordinary Session of the African Commission on Human and Peoples' Rights, May 2007, Accra, Ghana.

African Commission's Working Group of Experts on Indigenous Populations/ Communities. „Report of the African Commission's Working Group of Experts on Indigenous Populations/Communities", DOC/OS(XXXIV)/345, 20. November 2003.

Asia Indigenous Peoples Pact. „IPs to withdraw from active participation in World Intellectual Property Organization (WIPO) Inter Governmental Committee“, 21. Februar 2012. Zugegriffen 14. Januar 2017. http:/ / aippnet.org/ipss-decide-to-withdraw-from-active-participation-inworld-intellectual-property-organization-wipo-inter-governmentalcommittee/.

Asian Development Bank. „The Bank’s Policy on Indigenous Peoples“, April 1998. Zugegriffen 13. Januar 2017. https://www.adb.org/documents/policy-indigenous-peoples.

Association of Southeast Asian Nations. „ASEAN Framework Agreement on Access to Biological and Genetic Resources (Draft Text)“, 24. Februar 2000. Zugegriffen am 24. Januar 2017. http://www.mabs.jp/countries/others/pdf/321e.pdf.

Bering Sea Tribunal of Arbitration. „Bering Sea Fur Seals Fisheries Arbitration (Great Britain v. United States)“. Moore's International Arbitration, 1893. „Berne Convention for the Protection of Literary and Artistic Works“, 9. September 1886. 331 UNTS 217. 
British Broadcasting Corporation. „France sends Roma back to Romania“, 20. August 2010. Zugegriffen 14. Januar 2017. http://www.bbc.co.uk/news/world-europe-11020429. . „France-Turkey Armenia ,genocide“ row reflected in media“, 24. Januar 2012. Zugegriffen 14. Januar 2017. http://www.bbc.co.uk/news/worldeurope-16702453.

Bund der Polen in Deutschland e.V. Zugegriffen 8. Januar 2017. http://www.zpwn.org.

Bundesamt für Kultur, Schweizerische Eidgenossenschaft. „Anerkennung als nationale Minderheit“, 14. August 2007. Zugegriffen 7. Januar 2017. http://archive.li/nAQQp.

Bundesministerium des Innern. „Stellungnahme der Bundesrepublik Deutschland zu der Stellungnahme des Beratenden Ausschusses zu dem Bericht über die Umsetzung des Rahmen Übereinkommens zum Schutz nationaler Minderheiten in der Bundesrepublik Deutschland“, Juli 2002. Zugegriffen 15. Januar 2017. http://www.bmi.bund.de/cae/servlet/contentblob/151718/publicationF ile/13224/.

Bundesrat der Schweizerischen Eidgenossenschaft, „Botschaft über das Rahmenübereinkommen des Europarates zum Schutz nationaler Minderheiten“, BBl 1998 II, 1293, 19. November 1997.

„Cartagena Protocol on Biosafety to the Convention on Biological Diversity“, 2226 UNTS 208, 29. Januar 2000.

Commission of the Cartagena Agreement („Andean Community“), „Decision 391 - Common Regime on Access to Genetic Resources“, Official Gazette of the Cartagena Agreement No. 213, 2. Juli 1996.

Commission on Human Rights, „Study of the Legal Validity of the Undertaking concerning Minorities“, UN Doc. E/CN.4/367, 7. April 1950.

- . "Ninth Session, Summary Record of the 371st Meeting“, UN Doc. E/CN.4/SR.371, 5. Oktober 1953.

,Advisory Services in the Field of Human Rights, Report on the United Nations Seminar on the effect of racism and racial discrimination on the social and economic relations between indigenous peoples and States", UN Doc. E/CN.4/1989/22, 8. Februar 1989.

—. „Fourty eighth Session, Summary Record of the 17th Meeting“", UN Doc. E/CN.4/1992/SR.17, 12. Februar 1992.

„Forty-eighth session, Summary Record of the 38th Meeting“, UN Doc. E/CN.4/1992/SR.38, 10. April 1992.

-. „Summary Record of the 54th Meeting“, UN Doc. E/CN.4/1992/SR.54, 16. April 1992.

- . ,Rights of Persons belonging to National or Ethnic, Religious and Linguistic Minorities“, UN Doc. E/CN.4/1993/85, 10. Februar 1993. 
-. „Report of the Sub-Commission on the Prevention of Discrimination and Protection of Minorities on its Forty-Sixth Session“, UN Doc. E/CN.4/1995/2“, 28. Oktober 1994. . „Report on the Sixty-First Session“, UN Doc. E/CN.4/2005/135, 16. Dezember 2005.

Conference for Security and Co-operation in Europe. „Helsinki Document 1992, The Challenges of Change“, 9. Juli 1992. Zugegriffen 20. Januar 2017. http://www.osce.org/mc/39530.

Conference of the Parties to the Convention on Biological Diversity. „The implications of the international treaty on plant genetic resources for food and agriculture on the issues under Article 8(j) and related provisions“, $\mathrm{UNEP} / \mathrm{CBD} / \mathrm{COP} / 7 / \mathrm{INF} / 18,17$. Dezember 2003. , „Nagoya Protocol on Access to Genetic Resources and the Fair and Equitable Sharing of Benefits Arising from their Utilization to the Convention on Biological Diversity" (Nagoya Protocol on Access and Benefit-Sharing), UNEP/CBD/COP/DEC/X/1, 29. Oktober 2010.

- „Decision adopted by the Conference of the Parties to the Convention on Biological Diversity at its Tenth Meeting, X/43. Multiyear programme of work on the implementation of Article 8(j) and related provisions of the Convention on Biological Diversity“", $\mathrm{UNEP} / \mathrm{CBD} / \mathrm{COP} / \mathrm{DEC} / \mathrm{X} / 43$, 29. Oktober 2010. . „Report of the Seventh Meeting of the Ad Hoc Open-Ended Inter Sessional Working Group on Article 8(j) and Related Provisions of the Convention on Biological Diversity“", UNEP/CBD/COP/11/7, 24. November 2011.

„Convention For The Regulation Of Whaling“, 155 LNTS 349, 24. September 1931.

„Convention on Biological Diversity“, 1760 UNTS 79, 5. Juni 1992.

„Convention on the Prevention and Punishment of the Crime of Genocide“, 78 UNTS 277, 9. Dezember 1948.

Council of Scientific and Industrial Research. ,'Traditional Knowledge Digital Library“. Zugegriffen 24. Januar 2017.

http://www.csir.res.in/External/Utilities/Frames/career/main_page1.as $\mathrm{p} ? \mathrm{a}=$ tkdl_topframe.htm\&b=tkdl_left.htm\&c=../../../Heads $/$ TKDL $/$ mai n.htm.

Department of the Interior, Bureau of Indian Affairs. „Procedures for establishing that an American Indian Group exists as an Indian Tribe“, 59 FR 9293, 25. Februar 1994, 25 C.F.R. 83.7, „Mandatory criteria for Federal acknowledgment".

. „Final Determination for Federal Acknowledgment of the Shinnecock Indian Nation“, 75 FR 34760, 18. Juni 2010.

. „Indian Entities Recognized and Eligible To Receive Services From the United States Bureau of Indian Affairs“, 81 FR 26826, 4. Mai 2016. 
„Deutsche Reichstagsakten unter Kaiser Karl V. Der Reichstag zu Augsburg 1555“, abgedruck in: Aulinger, Rosemarie, Erwein H. Eltz, Ursula Machoczek, Eike Wolgast, und Bayerische Akademie der Wissenschaften, (Hrsg.) Deutsche Reichstagsakten, Jüngere Reihe Bd. 20, Teilbd. 4, 2009.

Dokumentations- und Kulturzentrum Deutscher Sinti und Roma. „Bürgerrechtsbewegung“. Zugegriffen 7. Januar 2017.

http://www.sintiundroma.de/no_cache/sinti-roma/buergerrechts bewegung.html?sword_list $\% 5 \mathrm{~B} 0 \% 5 \mathrm{D}=$ bürgerrechtsbewegung.

Economic and Social Council. „1982/34 Study of the Problem of Discrimination against Indigenous Populations“, E/1982/82, 7. Mai 1982.

—. „2000/22 Establishment of a Permanent Forum on Indigenous Issues“, UN Doc. E/2000/Inf/2/Add.2, 15. August 2000.

Economic and Social Council, Permanent Forum on Indigenous Issues. „,1589 (L) The problem of indigenous populations“, UN Doc. E/5044, 21. Mai 1971.

. „Attendance at the ninth session of the Permanent Forum on Indigenous Issues“, UN Doc. E/C.19/2011/INF/1, 2. Juni 2011.

EurActiv. „Slovakia-Hungary row over citizenship law“, 27. Januar 2011. Zugegriffen am 24. Januar 2017. http://www.euractiv.com/section/languagesculture/news/slovakia-hungary-row-over-citizenship-law/.

Europäische Gemeinschaft. „Declaration on the 'Guidelines on the Recognition of New States in Eastern Europe and in the Soviet Union"“, 31 ILM 1485, 16. Dezember 1991.

Europäischer Gerichtshof für Menschenrechte. „Noack and Others v. Germany (46346/99)“, Reports of Judgments and Decisions / Recueil des Arrêts et Décisions, 2000-VI, 25. Mai 2000.

- . „Case of Gorzelik and other v. Poland (44158/98), Grand Chamber“, Reports of Judgments and Decisions / Recueil des Arrêts et Décisions, 2004-I, 17. Februar 2004.

„European Charter for Regional or Minority Languages“ (Europäische Charta der Regional- und Minderheitensprachen), CETS 148, 5. November 1992.

Expert Group Meeting of Local Community Representatives within the Context of Article 8(j) and Related Provisions of the Convention on Biological Diversity. „Guidance for the Discussions Concerning Local Communities within the Context of the Convention on Biological Diversity“", UNEP/CBD/AHEG/LCR/2, 7. Juli 2011.

Food and Agricultural Organization. „Resolution 5/89, Farmers’ Rights“, Report of the Conference of FAO, 25nd Session, Rom 11.-29. November 1989, V. C 89/REP, 29. November 1989.

,International Treaty on Plant Genetic Resources for Food and Agriculture“, 2400 UNTS 379, 3. November 2001. 
„Framework Convention for the Protection Of National Minorities“ (Rahmenübereinkommen zum Schutz nationaler Minderheiten, FCNM), 2151 UNTS 243, 1. Februar 1995.

Frankfurter Allgemeine Zeitung. „Interesse an ungarischen Pässen“, 15. Februar 2011.

Hakim, Danny. „U.S. Recognizes Shinnecock Tribe on Long Island“, New York Times, 15. Juni 2010. Zugegriffen am 21. Januar 2017. http:/ /www.nytimes.com/2010/06/16/nyregion/16shinnecock.html?hp.

Human Rights Committee. „General Comment No. 23: The rights of minorities (Art. 27)“, CCPR/C/21/Rev.1/Add.5, 8. April 1994.

-. „Rules of Procedure of the Human Rights Committee“, CCPR/C/3/Rev.3, 24. Mai 1994.

Human Rights Council. „Institution-Building of the United Nations Human Rights Council“, UN Doc. A/HRC/RES/5/1, 18. Juni 2007.

. „Forum on Minority Issues“, UN Doc. A/HRC/RES/6/15, 28. September 2007.

Instituto do Patrimônio Histórico e Artístico Nacional. „Banco de Dados de Bens Culturais Imateriais Registrados“. Zugegriffen 13. Januar 2017. http://portal.iphan.gov.br/pagina/detalhes/228.

Instituto Nacional de Defensa de la Competencia y de la Protección de la Propiedad Intelectual. „Registro de Conocimientos Colectivos de Pueblos Indígenas“. Zugegriffen 13. Januar 2017. http://servicio.indecopi.gob.pe/portalctpi/index.jsp.

Interior Alliance Indigenous Nations. Brief an die World Trade Organization, Mr. Dariusz Rosati, „United States - Preliminary Determinations with Respect to Certain Softwood Lumber from Canada, WT/DS236“, 15. April 2002. Zugegriffen am 20. Januar 2017. http:/ / www.turtleisland.org/news/ia-wto.pdf.

International Centre for Trade and Sustainable Development. „WTO Members Comment on Indigenous Amicus Brief in Lumber Dispute“, 16. Mai 2002. Zugegriffen 13. Januar 2017. http://ictsd.org/i/news/biores/9174/.

„International Convention on the Elimination of All Forms of Racial Discrimination" (Internationales Übereinkommen zur Beseitigung jeder Form von Rassendiskriminierung), 660 UNTS 195, 7. März 1966.

„International Covenant on Civil and Political Rights“ (Internationaler Pakt über die bürgerlichen und politischen Rechte, IPbpR), 999 UNTS 171, 16. Dezember 1966.

International Labour Organization. „International Labour Organization Constitution“, 15 UNTS 40, 28. Juni 1919.

-. „Convention concerning the Protection and Integration of Indigenous and Other Tribal and Semi-Tribal Populations in Independent Countries“" (ILO Convention No. 107), 328 UNTS 247, 26. Juni 1957. 
. „Convention concerning Indigenous and Tribal Peoples in Independent Countries“ (ILO Convention No. 169), 28 ILM 1382, 27. Juni 1989.

International Whaling Comission. „Aboriginal subsistence whaling“. Zugegriffen 13. Januar 2017. https://iwc.int/aboriginal.

—. „Aboriginal Subsistence Whaling Catches Taken“. Zugegriffen 14. Januar 2017. https://iwc.int/table_aboriginal.

Intergovernmental Committee of the Universal Copyright Convention as Revised at Paris on 24 July 1971 und International Union for the Protection of Literary and Artistic Works, Executive Committee. „Consideration of the possibility of establishing an international instrument for the protection of folklore“, B/EC/IX/11 und IGC/XR.1(1971)15, 10. Dezember 1975.

Ludwig XIV und Ferdinand III. Westfälischer Friede - Vertrag von Münster (Instrumentum Pacis Monasteriensis), 1649.

Office of the High Commissioner for Human Rights. „United Nations Guide for Indigenous Peoples, Leaflet No. 10: Indigenous Peoples and the Environment", 2001.

„Optional Protocol to the International Covenant on Civil and Political Rights“, 999 UNTS 302, 16. Dezember 1966.

Organisation for Security and Co-operation in Europe (OSZE, vormals Conference on Security and Co-operation in Europe, CSCE). „Document of the Copenhagen Meeting of the Conference on the Human Dimension of the CSCE“, 29. Juni 1990. Zugegriffen am 21. Januar 2017. http://www.osce.org/odihr/elections/14304?download=true.

Organisation of the African Unity. ,Model Legislation for the Recognition and Protection of the Rights of Local Communities, Farmers and Breeders, and for the Regulation of Access to Biological Resources“. Gebilligt im Rahmen der 74. Sitzung des Ministerrates der OAU vom 5.-8. Juli 2001, CM/Dec.44 (LXXIV), 2000. Zugegriffen am 21. Januar 2017. http://www.wipo.int/wipolex/en/text.jsp? file_id=252153.

- . „Fourteenth Annural Activity Report of the African Commission on Human and Peoples’ Rights“", AHG/229(XXXVII), 2.-12. Juli 2001, Resolution on the Rights of Indigenous People/Communities in Africa.

Permanent Court of International Justice. „Minority Schools in Albania“, 1935 PCIJ, Ser. A/B, No. 64, 6. April 1935.

_. „Greco-Bulgarian Communities“, 1930 PCIJ, Ser B, No. 17, 31. Juli 1930.

Rüb, Matthias. „Harper entschuldigt sich bei Ureinwohnern: Kanada weint nach der historischen Geste“, Frankfurter Allgemeine Zeitung, 6. Dezember 2008. „Satzung des Völkerbundes“, 225 CTS 188, 28. Juni 1919.

„Schlussakte der Wiener Ministerkonferenzen oder Bundes-Supplementar-Akte“ („Wiener Schlussakte“), 25. November 1918, in: Binding, Karl (Hrsg.), Deutsche Staatsgrundgesetze, Teil 3: Konföd.-, Deutsche Bundes-, Wiener Schlußakte, 1909. 
Sub-Commission on the Prevention of Discrimination and Protection of Minorities. Mr. Hernán Santa Cruz, ,Special Study of Racial Discrimination in the Political, Economic, Social and Cultural Spheres“, UN Doc. E/CN.4/Sub.2/301, 24. Juni 1969.

- . „Report of the Twenty-Fourth Session of the Sub-Commission on the Prevention of Discrimination and Protection of Minorities to the Commission on Human Rights, Resolution 8 (XXIV)", UN Doc. E/CN.4/Sub.2/323, 6. Oktober 1971.

. Francesco Capotorti, „Study on the rights of persons belonging to ethnic, religious, and linguistic minorities“, UN Doc. E/CN.4/Sub.2/384/Rev.1, 1979.

- Jules Deschênes, „Proposal concerning a definition of the term ,minority“،, UN. Doc. E/CN.4/Sub.2/1985/31, 14. Mai 1985.

. José R. Martínez Cobo, „Study of the Problem of Discrimination against Indigenous Populations“, einzelne Berichte zwischen 1981-1983, zusammengefasst neu veröffentlich unter: UN Doc. E/CN.4/Sub.2/1986/7 und Add.1-4.

. Asbjørn Eide, „Preliminary Report submitted by the Special Rapporteur Mr. Asbjørn Eide, on possible ways and means of facilitating the peaceful and constructive solution of problems involving minorities", UN Doc. E/CN.4/Sub.2/1990/46, 20. Juli 1990.

. Asbjørn Eide, „Possible Ways and Means of Facilitating the Peaceful and Constructive Solution of Problems Involving Minorities“, UN Doc.

E/CN.4/Sub.2/1993/34 and Add. 1-4, 10. August 1993.

. „Working Paper by the Chairperson - Rapporteur, Mrs. Erica-Irene A.

Daes on the concept of ,indigenous people“", UN Doc.

E/CN.4/Sub.2/AC.4/1996/2, 10. Juni 1996.

. Erica-Irene Daes, „Indigenous peoples and their relationship to land“, Final working paper prepared by the Special Rapporteur, Mrs. Erica-Irene A. Daes, UN Doc. E/CN.4/Sub.2/2001/21, 11. Juni 2001.

Süddeutsche Zeitung. „Ureinwohner in Australien - Historische Entschuldigung“, 17. Mai 2010.

Survival International. „Unkontaktierte Völker Brasiliens“. Zugegriffen 3. Januar 2017.

http://www.survivalinternational.de/indigene/unkontaktiertebrasilien.

Tebtebba. „Final Statement and Question from Indigenous Peoples Major Group“, 11. April 2012. Zugegriffen 20. Dezember 2016.

http:/ / www.tebtebba.org/index.php/content/211-final-statement-andquestion-from-indigenous-peoples-major-group.

„Traité de paix signé à Paris le 30 mars 1856 entre la Sardaigne, l’Autriche, la France, le Royaume Uni de la Grande Bretagne et d'Irlande, la Prusse, la Russie et la Turquie“. 114 CTS, 409, 30. März 1856. 
„Treaty on Friendship and Cooperation between the Republic of Hungary and the Republic of Slovakia“, Paris, 19. März 1995, abgedruckt in: Arp, Björn (Hrsg.), International Norms and Standards for the Protection of National Minorities, 2008.

„Übereinkommen zur Erhaltung der wandernden wild lebenden Tierarten (Bonner Übereinkommen)“, 1651 UNTS 333, 23. Juni 1979.

UN Department of Economic and Social Affairs. „How to Apply for Consultative Status“. Zugegriffen am 8. Januar 2017. http:// csonet.org/?menu=83.

UN Generalversammlung, ,International Bill of Human Rights“, UN Doc. A/Res/3/217(III)C, 10. Dezember 1948.

- . „Report of the Human Rights Committee“, Communication No. R.6/24, Sandra Lovelace v. Canada (Lovelace v. Canada), UN Doc. A/36/40 (Supp), 29. September 1981.

-. „Report of the Human Rights Committee“, Communication No. 78/1980, The Mikmaq Tribal Society v. Canada, UN Doc.

A/39/40(Supp), 20. September 1984.

. World Commission on Environment and Development, „Our common future“ („Brundtland Report“), UN Doc. A/42/427, 4. August 1987.

„Report of the Human Rights Committee“, Communication No.

197/1985, Kitok v. Sweden, UN Doc. A/43/40(Supp), 28. September 1988.

„Report of the Human Rights Committee“, Communication No.

167/1984, Bernard Ominayak, Chief of the Lubicon Lake Band v. Canada (Lubicon Lake Band v. Canada), UN Doc. A/45/40(Vol.II)(Supp), 4. Oktober 1990.

„Rio Declaration on Environment and Development“, UN Doc. A/CONF.151/26(Vol. I), 12. August 1992.

„Declaration on the Rights of Persons Belonging to National or Ethnic, Religious and Linguistic Minorities“, UN Doc. A/Res/47/135, 18. Dezember 1992.

„Report of the Human Rights Committee“, Communication Nos. 359/1989 and 385/1989, John Ballantyne and Elizabeth Davidson, and Gordon McIntyre v. Canada (Ballantyne et al. v. Canada), UN Doc. A/48/40(PARTII), 1. November 1993.

- . „High Commissioner for the Promotion and Protection of all Human Rights“, UN Doc. A/Res/48/141, 20. Dezember 1993.

Intergovernmental Negotiating Committee for the Elaboration of an International Convention to Combat Desertification in those Countries Experiencing Serious Drought and/or Desertification, Particularly in Africa, „United Nations Convention to Combat Desertification in those Countries Experiencing Serious Drouhgt and/or Desertification, Particularly in Africa“" (Convention to Combat Desertification), UN Doc. A/AC.241/27, 12. September 1994. 
. „Report of the Human Rights Committee“, Communication No. 760/1997, J.G.A. Diergaardt et al. v. Namibia, UN Doc.

A/55/40[Vol.II](SUPP), 18. Oktober 2000.

. „Human Rights Council“, UN Doc. A/Res/60/251, 3. April 2006.

. "Working group of the Commission on Human Rights to elaborate a draft declaration in accordance with paragraph 5 of General Assembly resolution 49/214 of 23 December 1994“, UN Doc. A/Res/61/178“, 6. März 2007.

. „United Nations Declaration on the Rights of Indigenous Peoples“, UN Doc. A/Res/61/295, 13. September 2007.

—. „The future we want“, UN Doc. A/Res/66/288“, 11. September 2012.

United Nations Conference on Environment and Development. „Report of the United Nations Conference on Environment and Developtment", Annex II, Agenda 21, UN. Doc. A/CONF.151/26/Rev.1 (Vol. I), 3.-14. Juni 1992.

. „Report of the United Nations Conference on Environment and Developtment", Annex III, Non-legally binding Authoritative Statement of Principles for a Global Consensus on the Management, Conservation and Sustainable Development of all Types of Forests (Forest Principles), UN Doc. A/CONF.151/26(Vol. III), 14. August 1992.

United Nations Educational, Scientific, and Cultural Organization. „Declaration on Race and Racial Prejudice“, UN Doc. E/CN.4/Sub.2/1982/2/Add.1, Annex V, 27. November 1978.

United Nations Educational, Scientific, and Cultural Organization und World Intellectual Property Organization. „Model Provisions for National Laws on the Protection of Expressions of Folklore Against Illicit Exploitation and other Forms of Prejudicial Action, with a commentary prepared by the Secretariats of UNESCO and WIPO“, 1985. Zugegriffen am 21. Januar 2017.

http://www.wipo.int/edocs/lexdocs/laws/en/unesco/unesco001en.pdf, und http:// unesdoc.unesco.org/images/0006/000684/068457mb.pdf. . „Tunis Model Law on Copyright for Developing Countries“, 2. März 1976. WIPO Publication No. 812 (E). Zugegriffen am 23. Januar 2017. http://portal.unesco.org/culture/en/ev.php-

URL_ID=31318\&URL_DO=DO_TOPIC\&URL_SECTION=201.html.

United Nations Permanent Forum on Indigenous Issues. „Handbook for Participants“, 2007. Zugegriffen am 24. Januar 2017.

www.un.org/esa/socdev/unpfii/documents/handbook_participants_en. pdf.

US Department of the Interior, Office of the Assistant Secretary - Indian Affairs (Press Release). „Echo Hawk Issues Decisions on Two Tribal Gaming Applications“, 20. Dezember 2011. Zugegriffen am 21. Januar 2017. 
https://indianaffairs.gov/cs/groups/public/documents/text/idc015848. pdf.

US Congress. „The Federally Recognized Indian Tribe List Act of 1994“, Public Law 103-454, 108 Stat. 4791, 2. November 1994.

„Vertrag zwischen Deutschland, Österreich-Ungarn, Frankreich, Großbritannien, Italien, Rußland und der Türkei“" (Berliner Vertrag). Deutsches Reichsgesetzblatt, Band 1878, Nr. 31, S. 307-345, 13. Juli 1878.

World Intellectual Property Organization. Committee of Experts to a Possible Protocol to the Berne Convention und Committee of Experts on a Possible Instrument for the Protection of the Rights of Performers and Producers of Phonograms. „Report adopted by the committees“, BCP/CE/VI/16 - INR/CE/V/14, 9. Februar 1996.

. „The Protection of Expressions of Folklore: The Attempts at International Level“", Intellectual Property in Asia and the Pacific, Nr. 56/57 (1998).

„Roundtable on Intellectual Property and Indigenous Peoples“, WIPO/INDIP/RT/98, 23.-24. Juli 1998.

-. „WIPO-UNESCO African Regional Consultation on the Protection of Expressions of Folklore“, WIPO-UNESCO/FOLK/AFR/99, 23.-25. März 1999.

—. „The Protection of Expressions of Folklore: The Attempts at International Level“, WIPO/ACAD/E/99/12, 1. April 1999.

. „WIPO-UNESCO Regional Consultation on the Protection of Expressions of Folklore for Countries of Asia and the Pacific", WIPO-

UNESCO/FOLK/ASIA/99, 21.-23. April 1999.

. „WIPO-UNESCO Regional Consultation on the Protection of Expressions of Folklore for Arab Countries“, WIPO-

UNESCO/FOLK/ARAB/99, 25.-27. Mai 1999.

. „WIPO-UNESCO Regional Consultation on the Protection of Expressions of Folklore for Latin America and the Caribbean“, WIPO-

UNESCO/FOLK/LAC/99, 14.-16. Juni 1999.

„Roundtable on Intellectual Property and Traditional Knowledge“, WIPO/IPTK/RT/99, 1.-2. November 1999.

. Intellectual Property Needs and Expectations of Traditional Knowledge Holders, WIPO Report on Fact-finding Missions on Intellectual Property and Traditional Knowledge (1998-1999), 2001.

„Key Issues, Gap Analysis and Consultations“. Zugegriffen 14. Januar 2017. http://www.wipo.int/tk/en/igc/consultations.html.

- „Draft Provisions/Articles for the Protection of Traditional Knowledge and Traditional Cultural Expressions, and IP \& Genetic Resources“.

Zugegriffen 14. Januar 2017.

http://www.wipo.int/tk/en/igc/draft_provisions.html. 
WIPO Generalversammlung. ,Matters Concerning Intellectual Property and Genetic Resources, Traditional Knowledge and Folklore“, WO/GA/26/6, 25. August 2000.

. ,Twenty-Sixth (12th Extraordinary) Session Geneva, September 25 to October 3, 2000, Report“", WO/GA/26/10, 3. Oktober 2000.

. ,Thirty-Eighth (19th Ordinary) Session, Geneva, September 22 to October 1, 2009, Report“, WO/GA/38/20, 1. Oktober 2009.

. „Establishment of the WIPO Voluntary Fund for Accredited Indigenous and Local Communities", Annex zu WO/GA/32/6, 24. August 2005, geändert durch WO/GA/39/11, 5. Juni 2010.

. „Matters Concerning the Intergovernmental Committee on Intellectual

Property and Genetic Resources, Traditional Knowledge and Folklore (IGC)“, WO/GA/40/7, 12. August 2011.

_. „Matters Concerning the Intergovernmental Committee on Intellectual Property and Genetic Resources, Traditional Knowledge and Folklore (IGC)“, WO/GA/41/15, 1. August 2012.

WIPO Intergovernmental Committee. „Matters Concerning Intellectual Property and Genetic Resources, Traditional Knowledge and Folklore - An Overview“, WIPO/GRTKF/IC/1/3, 16. März 2001.

. „Traditional Knowledge and the Need to Give it Adequate Intellectual Property Protection. WIPO Committee on the Relationship between Intellectual Property, Genetic Resources and Traditional Knowledge (documents submitted by the Group of Countries of Latin America and the Caribbean (GRULAC)“, WIPO/GRTKF/IC/1/5, 16. März 2001.

. „Document of the Holy See on Intellectual Property and Genetic Re-

sources, Traditional Knowledge and Folklore“, WIPO/GRTKF/IC/1/7, 26. April 2001.

- „Proposal Presented by the African Group to the First Meeting of the Intergovernmental Committee on Intellectual Property and Genetic Resources, Traditional Knowledge and Folklore (submitted by the African Group)“, WIPO/GRTKF/IC/1/10, 1. Mai 2001.

. „First Session, April 30 to May 3, 2001, Report“, WIPO/GRTKF/IC/1/13, 23. Mai 2001.

. „Position Paper of the Asian Group and China (submitted by the Asian Group and China)“, WIPO/GRTKF/IC/2/10, 3. Dezember 2001. . „Final Report on National Experiences with the Legal Protection of Expressions of Folklore“, WIPO/GRTKF/IC/3/10, 25. März 2002. . „Expressions of Folklore, Document submitted by the European Community and its Member States", WIPO/GRTKF/IC/3/11, 16. Mai 2002. - „Participation of Local and Indigenous Communities in the Work of the Committee“, WIPO/GRTKF/IC/4/12, 20. Oktober 2002. . „Traditional Cultural Expressions/Expressions of Folklore Legal and Policy Options“, WIPO/GRTKF/IC/6/3, 1. Dezember 2003. 
-. „Traditional Knowledge: Policy and Legal Options“, WIPO/GRTKF/IC/6/4, 12. Dezember 2003. „Sixth Session, March 15 to 19, 2004, Report“, WIPO/GRTKF/IC/6/14, 14. April 2004.

-. „The Protection of Traditional Cultural Expressions/Expressions of Folklore: Overview of Policy Objectives and Core Principles“, WIPO/GRTKF/IC/7/3, 20. August 2004.

- „Protection of Traditional Knowledge: Overview of Policy Objectives and Core Principles“, WIPO/GRTKF/IC/7/5, 20. August 2004. „Decisions adopted by the Committee at it's Seventh Session, Geneva, November 1 to 5, 2004“, WIPO/GRTKF/IC/7/Decisions, 5. November 2004. . „Overview of the Committee’s Work on Genetic Resources“, WIPO/GRTKF/IC/8/9, 20. April 2005.

„Decisions of the Ninth Session of the Committee“, WIPO/GRTKF/IC/9/DECISIONS, 8. April 2006.

- „The Protection of Traditional Cultural Expressions/Expressions of Folklore: Draft Objectives and Principles“, WIPO/GRTKF/IC/10/4, 2. Oktober 2006.

- , "The Protection of Traditional Knowledge: Draft Objectives and Principles“, WIPO/GRTKF/IC/10/5, 2. Oktober 2006.

—. "Tenth Session, November 30 to December 8, 2006, Report“, WIPO/GRTKF/IC/10/7 Prov.2, 25. April 2007.

- ,The Protection of Traditional Cultural Expression/Expression of Folklore: Factual Extraction“, WIPO/GRTKF/IC/12/4(B), 31. Januar 2008.

- . „The Protection of Traditional Knowledge: Factual Extraction“, WIPO/GRTKF/IC/12/5(B), 18. Februar 2008.

- .Eleventh Session, July 3 to July 12, 2007, Report“, WIPO/GRTKF/IC/11/15, 20. März 2008.

- . „The Protection of Traditional Cultural Expressions: Draft Gap Analysis“, WIPO/GRTKF/IC/13/4(B) REV., 11. Oktober 2008.

- . "Twelfth Session, February 25 to 29, 2008, Report“, WIPO/GRTKF/IC/12/9, 21. November 2008.

- „Proposal of Group B and Group of Central European and Baltic States for Intersessional Working Groups for the Intergovernmental Committee“, WIPO/GRTKF/IC/15/6, 11. Dezember 2009.

. ,The Protection of Traditional Cultural Expressions/ Expressions of Folklore: Revised Objectives and Principles“, WIPO/GRTKF/IC/16/4, 22. März 2010.

,The Protection of Traditional Knowledge: Revised Objectives and Principles“, WIPO/GRTKF/IC/16/5, 22. März 2010.

-. „Submission by Australia, Canada, New Zealand, Norway and the United States of America“, WIPO/GRTKF/IC/16/7, 6. Mai 2010. 
. „Fifteenth Session, December 7 to 11, 2009, Report“", WIPO/GRTKF/IC/15/7, 14. Mai 2010.

. ,The Protection of Traditional Cultural Expressions/Expressions of

Folklore: Revised Objectives and Principles“, WIPO/GRTKF/IC/17/4, 21. September 2010.

. „Sixteenth Session, May 3 to May 7, 2010, Draft Report“,

WIPO/GRTKF/IC/16/8 Prov.2, 30. September 2010.

- . „The Protection of Traditional Knowledge: Revised Objectives and Principles“, WIPO/GRTKF/IC/18/5, 10. Januar 2011.

. „Draft Objectives and Principles Relating to Intellectual Property and

Genetic Resources prepared at IWG 3“, WIPO/GRTKF/IC/18/9,

17. März 2011.

. „Seventeenth Session, December 6 to 10, 2010, Report“, WIPO/GRTKF/IC/17/12, 6. Juni 2011.

. „Eighteenth Session, May 9 to 13, 2011, Report“,

WIPO/GRTKF/IC/18/11, 29. Juli 2011.

-. "Nineteenth Session, July 18 to 22, 2011, Report“, WIPO/GRTKF/IC/19/12, 23. Februar 2012.

. „Fifteenth Session, December 7 to 11, 2009, Report“,

WIPO/GRTKF/IC/15/7, 14. Mai 2010.

. „Glossary of Key Terms Related to Intellectual Property and Traditional Cultural Expressions“, WIPO/GRTKF/IC/19/INF/7, 17. Mai 2011.

. „The Protection of Traditional Cultural Expressions: Draft Articles“, WIPO/GRTKF/IC/19/4, 17. Mai 2011.

."The Protection of Traditional Knowledge: Draft Articles“,

WIPO/GRTKF/IC/19/5, 20. Mai 2011.

. „Recommendations of the Second Session of Like Minded Countries

Meeting on the Protection of Genetic Resources, Traditional Knowledge and Folklore“, WIPO/GRTKF/IC/19/8, 8. Juli 2011.

- „Like-Minded Countries Contribution to the Draft Articles on the Protection of Traditional Cultural Expressions“, WIPO/GRTKF/IC/19/9, 18. Juli 2011.

. „Like-Minded Countries Contribution to the Draft Articles on the Protection of Traditional Knowledge“, WIPO/GRTKF/IC/19/10, 18. Juli 2011.

- „Like-Minded Countries Contribution to the Objectives and Principles on the Protection of Genetic Resources and Preliminary Draft Articles on the Protection of Genetic Resources“, WIPO/GRTKF/IC/19/11, 18. Juli 2011.

, Options for Future Work on Intellectual Property and Genetic Resources“, WIPO/GRTKF/IC/20/5, 10. Oktober 2011. 
- . „Glossary of Key Terms Related to Intellectual Property and Genetic Resources, Traditional Knowledge and Traditional Cultural Expressions“ (Glossar), WIPO/GRTKF/IC/20/INF/13, 7. Dezember 2011.

-. "The Protection of Traditional Knowledge: Draft Articles“, WIPO/GRTKF/IC/21/4, 18. Januar 2012.

- . „The Protection of Traditional Cultural Expressions: Draft Articles“, WIPO/GRTKF/IC/22/4, 27. April 2012.

-. „Accredited observers to the WIPO Intergovernmental Committee on Intellectual Property and Genetic Resources, Traditional Knowledge and Folklore (IGC)“. Zugegriffen am 24. Januar 2017. http://www.wipo.int/export/sites/www/tk/en/igc/pdf/igc_observers.p df.

„Participating in the IGC“. Zugegriffen am 24. Januar 2017. http://www.wipo.int/tk/en/igc/participation.html.

- . „Protection of Traditional Cultural Expressions and Traditional Knowledge - Gap Analyses“. Zugegriffen 24. Januar 2017. http://www.wipo.int/tk/en/igc/gap-analyses.html.

- . „Protection of Traditional Cultural Expressions and Traditional Knowledge - Key Issues“. Zugegriffen 24. Januar 2017. http://www.wipo.int/tk/en/igc/issues.html.

World Summit on Sustainable Development. „Plan of Implementation of the World Summit on Sustainable Development“", UN Doc. A/Conf.199/20, 4. September 2002.

„The Johannesburg Declaration on Sustainable Development: From Our Origins to the Future“, UN Doc. A/Conf.199/20, 4. September 2002. 


\section{B. Sekundärquellen: Monographien, Artikel, Beiträge in Sammelwerken}

Abraham, Meghna. A new chapter for human rights: a handbook on issues of transition from the Commission on Human Rights to the Human Rights Council, 2006.

Alfredsson, Gudmundur. „Minority Rights at the United Nations“. In Managing diversity: protection of minorities in international law, herausgegeben von Daniel Thürer und Zdzisław Kedzia, 2009.

Anaya, S. James. Indigenous peoples in international law, 2004.

Anghie, Antony. Imperialism, sovereignty and the making of international law, 2005.

Arp, Björn. International norms and standards for the protection of national minorities, 2008.

Backiny-Yetna, Prospere, und Quentin Wodon. „Census-based profile of the

Mbororos and the Pygmies in the Central African Republic", World Bank

- Central African Republic Country Brief No. 8, 1. Mai 2011.

-. „Census-based profile of the Pygmies in Gabon“, World Bank - Gabon Country Brief No. 5, 1. März 2011.

Baldwin, Clive, und Cynthia Morel. „Using the United Nations Declaration on the Rights of Indigenous Peoples in Litigation". In Reflections on the UN Declaration on the Rights of Indigenous Peoples, herausgegeben von Steve Allen und Alexandra Xanthaki, 2011.

Barsh, Russel Lawrence. „Indigenous Peoples“. In The Oxford handbook of international environmental law, herausgegeben von Daniel Bodansky, Jutta Brunnée, und Ellen Hey, 2007.

. „Indigenous Peoples in the 1990s: From Object to Subject in International Law?" Harvard Human Rights Journal, Vol. 7 (1994), S. 33-86.

Barth, Fredrik. Ethnic groups and boundaries. The social organization of culture difference, 1969.

Bebbington, Anthony. „Modernization from Below: An Alternative Indigenous Development?" Economic geography, Vol. 69, Nr. 3 (1993), S. 274-292.

Bedorf, Thomas. „Interkulturelle Anerkennung, die Verkennung der Identität und der postkoloniale Diskurs", Vortrag auf dem XXI deutschen Kongress für Philosophie ,Lebenswelt und Wissenschaft', 15. September 2008. Zugegriffen 15. Januar 2017.

http://www.dgphil2008.de/fileadmin/download/Sektionsbeitraege/081_Bedorf.pdf.

- Verkennende Anerkennung: Über Identität und Politik, 2010.

Ben-Achour, Arbi, Propere Backiny-Yetna und Quentin Wodon, „Socioeconomic status of the Pygmies in the Democratic Republic of Congo“, World Bank - Democratic Republic of Congo Country Brief No. 6, 1. April 2011. 
Berkes, Fikret. „Traditional Ecological Knowledge in Perspective“. In Traditional Ecological Knowledge: Concepts and Cases, herausgegeben von Julian Inglis, 1993.

Berman, Howard R. „The International Labour Organization and Indigenous Peoples: Revision of ILO Convention No. 107 at the 75th Session of the International Labour Converence, 1988“. The Review (International Commission of Jurists), Vol. 41 (1988), S. 48-57.

Beyerlin, Ulrich. „,Erhaltung und nachhaltige Nutzung“ als Grundkonzept der Biodiversitätskonvention". In 10 Jahre Übereinkommen über die biologische Vielfalt: eine Zwischenbilanz, herausgegeben von Nina Wolff und Wolfgang Köck, 2004.

Biber-Klemm, Susette, und Danuta Szymura Berglas. „General Framework, Problems and Goals“. In Rights to plant genetic resources and traditional knowledge: basic issues and perspectives, herausgegeben von Susette Biber-Klemm und Thomas Cottier, 2006.

Bloed, Arie. „The OSCE and the Issue of National Minorities“. In Universal Minority Rights, herausgegeben von Alan Phillips und Allan Rosas, 1995.

Borodin, R. G., V. M. Etylin, O. V. Etylina, D. Litovka, V. V. Melnikov, L. L. Solovenchuk, N. G. Shevchenko, E. V. Zdor, S. A. Blokhin, G. M. Zelensky und L. I. Ainana. „Cultural, Traditional and Nutritional Needs of the Aboriginal Population of Chukotka for Gray Whales and Bowhead Whales 2003-2007“, IWC/54/AS/5, 2002.

Boysen, Sigrid. Europäische Charta der Regional-oder Minderheitensprachen: Handkommentar, 2011.

Brölmann, Catherine, und Marjoleine Zieck. „Indigenous Peoples“. In Peoples and Minorities in International Law, herausgegeben von Catherine Brölmann, René Lefeber, und Marjoleine Zieck, 1993.

Brown, Michael Fobes. Who owns native culture?, 2004.

Brownlie, Ian. Principles of public international law, 2008.

-. „Recognition in theory and practice“. The British yearbook of international law, 1983, S. 197-211.

-. ,The Rights of Peoples in Modern International Law“. In The Rights of peoples, herausgegeben von James Crawford, 1988.

Brush, Stephen B., und Doreen Stabinsky. V aluing Local Knowledge: Indigenous People and Intellectual Property Rights, 1996.

Cabrera Ormaza, Maria Victoria. „Re-thinking the Role of Indigenous Peoples in International Law: New Developments in International Environmental Law and Development Cooperation“. Goettingen Journal of International Law Vol. 4, Nr. 1 (2012), S. 263-290.

Cassese, Antonio. Self-determination of peoples, 1996.

Castellino, Joshua. „Indigenous Rights and the Right to Development: Emerging Synergies or Collusion?" In Reflections on the UN Declaration on the Rights of 
Indigenous Peoples, herausgegeben von Steve Allen und Alexandra Xanthaki, 2011.

International law and self-determination, 2000.

. Minority rights in the Pacific region: a comparative analysis, 2009.

Centre for International Environmental Law (CIEL). A Citizen's Guide to WIPO, 2007.

Charters, Claire, und Rodolfo Stavenhagen (Hrsg). Making the Declaration work: the United Nations Declaration of the rights of indigenous peoples, 2009.

Cole, Mark D. Das Selbstbestimmungsrecht indigener Völker, 2009.

Coombe, Rosemary J. „Der zunehmende Geltungsbereich kultureller Besitztümer und ihrer Politik". In Die Konstituierung von Cultural Property: Forschungsperspektiven, herausgegeben von Regina Bendix, Stefan Groth, und Kilian Bizer, 2010.

The cultural life of intellectual properties: authorship, appropriation and the law, 1998.

Crawford, James. The creation of states in international law, 2006.

. The International Law Commission's articles on state responsibility, 2002.

. The Rights of peoples, 1988.

Curci, Jonathan. The protection of biodiversity and traditional knowledge in international law of intellectual property, 2010.

Daes, Erica-Irene. „,The Contribution of the Working Group on Indigenous Populations to the Genesis and Evolution of the UN Declaration on the Rights of Indigenous Peoples". In Making the Declaration Work, the United Nations Declaration on the Rights of Indigenous Peoples, 2009.

. „The UN Declaration on the Rights of Indigenous Peoples: Background and Appraisal". In Reflections on the UN Declaration on the Rights of Indigenous Peoples, herausgegeben von Steve Allen und Alexandra Xanthaki, 2011.

Dagne, Teshager Worku. Intellectual Property, Traditional Knowledge and Biodiversity in the Global Economy: The Potential of Geographical Indications for Protecting Traditional Knowledge-Based Agricultural Products, 2012.

Dang, Hai-Anh H. „Growth in Vietnam is strong but not shared equitably across ethnic groups“, World Bank - Vietnam Country Brief No. 1, 1. September 2009.

Das, Maitreyi Bordia, Gillette Hall, Soumya Kapoor und Denis Nikitin. „India’s Adivasis“, World Bank - India Country Brief No. 4, 1. Januar 2011.

de Vattel, Emer. Le droit des gens ou Principes de la loi naturelle appliqués à la conduite et aux affaires des nations et des souverains. Die Klassiker des Völkerrechts in modernen deutschen Übersetzungen, 1959.

de Vitoria, Francisco. De Indis recenter inventis et de jure belli hispanorum in barbaros, herausgegeben von Walter Schätzel, 1952.

Diaz, Augusto Willemsen. „How Indigenous Peoples’ Rights reached the UN“. In Making the Declaration Work, the United Nations Declaration on the Rights of In- 
digenous Peoples, herausgegeben von Claire Charters und Rodolfo Stavenhagen, 2009.

Drzewicki, Krzysztof. „Minority Protection within the OSCE“. In Managing diversity: protection of minorities in international law, herausgegeben von Daniel Thürer und Zdzisław Kedzia, 2009.

Eide, Asbjørn. „Historical and Global Perspectives“. In The Rights of Minorities: A Commentary on the European Framework. Convention for the Protection of National Minorities, herausgegeben von Marc Weller, 2005.

Engle, Karen. „On Fragile Architecture: The UN Declaration on the Rights of Indigenous Peoples in the Context of Human Rights“. European journal of international law, Vol. 22, Nr. 1 (2011), S. 141-164.

. The elusive promise of indigenous development: rights, culture, strategy, 2010.

Ermacora, Felix. Historische Entwicklung der Menschenrechte und Grundfreiheiten. Menschenrechte in der sich wandelnden Welt, 1974.

Fach, Estelle. „Legal Empowerment of Local Communities: a Role for International Environmental Law?" Zugegriffen 25. November 2014.

https://www.scps.nyu.edu/export/sites/scps/pdf/global-affairs/estellefach.pdf.

Fisch, Jörg. Die europäische Expansion und das Völkerrecht. Beiträge zur Kolonial- und Überseegeschichte, 1984.

Gilbert, Geoff. „Article 5“. In The Rights of Minorities: A Commentary on the European Framework Convention for the Protection of National Minorities, herausgegeben von Marc Weller, 2005.

Gilbert, Jérémie. „Indigenous peoples’ human rights in Africa: the pragmatic revolution of the African Commission on Humand and Peoples' Rights“. International \& Comparative Law Quarterly, Vol. 60, Nr. 1 (2011), S. 245-270. . Indigenous peoples' land rights under international law, 2006.

Gilbert, Jérémie, und Cathal Doyle. „A New Dawn over the Land: Shedding Light on Collective Ownership and Consent". In Reflections on the UN Declaration on the Rights of Indigenous Peoples, herausgegeben von Steve Allen und Alexandra Xanthaki, 2011.

Gillespie, Alexander. „Aboriginal Subsistence Whaling: A Critique of the InterRelationship Between International Law and the International Whaling Commission". Colorado Jounral of International Environmental Law and Policy, Vol. 12 (2001), S. 77-139.

Groth, Stefan. Negotiating Tradition - The Pragmatics of International Deliberations on Cultural Property. Göttinger Studien zu Cultural Property, 2011.

Gruenberg, Justin S. „An Analysis of United Nations Security Council Resolutions: Are All Countries Treated Equally?" Case Western Reserve Journal of International Law, Vol. 41, Nr. 2-3 (2009), S. 469-512.

Hall, Gillette, und Harry A. Patrinos. Indigenous Peoples, Poverty, and Development. 2014. 
Hannum, Emily. „Ethnic disparities in China: geography, rurality, and socioeconomic welfare“, World Bank - China Country Brief No. 3, 1. Januar 2011.

Hanski, Raija. Leading cases of the Human Rights Committee, 2007.

Haselhuber, Jakob. „Institutionalisierung ohne Verrechtlichung: der Hohe Kommissar für nationale Minderheiten der OSZE“. In Moderner Minderheitenschutz: rechtliche oder politische Absicherung?; Zum 50. Jahrestag der UNMenschenrechtserklärung, herausgegeben von Hans-Joachim Heintze, 1998.

Henrard, Kristin. „The Impact of the Enlargement Process on the Development of a Minority Protection Policy within the EU; Another Aspect of Responsibility/Burden-Sharing?" Maastricht Journal of European and Comparative law, Vol. 9, Nr. 4 (2002), S. 357-393.

Henriksen, John. „The UN Declaration on the Rights of Indigenous Peoples: Some Key Issues and Events in the Process". In Making the Declaration Work, the United Nations Declaration on the Rights of Indigenous Peoples, herausgegeben von Claire Charters und Rodolfo Stavenhagen, 2009.

Hillgruber, Christian. Die Aufnahme neuer Staaten in die Völkerrechtsgemeinschaft. Kölner Schriften zu Recht und Staat, 1998.

Hilpold, Peter. Modernes Minderheitenrecht: eine rechtsvergleichende Untersuchung des Minderheitenrechtes in Österreich und in Italien unter besonderer Berücksichtigung völkerrechtlicher Aspekte, 2001.

Hobe, Stephan. Einführung in das Völkerrecht, 2008.

Hofmann, Rainer. „The Framework Convention for the Protection of National Minorities“. In Minority Rights in Central and Eastern Europe, herausgegeben von Bernd Rechel, 2009.

Honneth, Axel. Kampf um Anerkennung: Zur moralischen Grammatik sozialer Konflikte, 2010.

Innozenz IV. Commentaria super libros quinque decretalium, 1570, Neudruck 1968.

Jenkins, Richard. Rethinking ethnicity: arguments and explorations, 1997.

John, Grand Chief Ed. „Remarks by the Chair of the United Nations Permanent Forum on Indigenous Issues, High-Level Event to Commemorate the 5th Anniversary of the Adoption of the UN Declaration on the Rights of Indigenous Peoples“, 17. Mai 2012. Zugegriffen 24. Januar 2017. http://www.un.org/esa/socdev/unpfii/documents/5th-annivundrip/chairperson-stmt.pdf.

Karl, Doehring. „Das Gutachten des Generalsekretärs der Vereinten Nationen über die Fortgeltung der nach dem ersten Weltkrieg eingegangenen Minderheitenschutzverpflichtungen“. Zeitschrift für ausländisches öffentliches Recht und Völkerrecht, Vol. 15, 1953, S. 521-540.

Keal, Paul. ,'Just Backward Children': International Law and the Conquest of Non-European Peoples“. In Indigenous Rights, herausgegeben von Anthony J Connolly, 2009. 
Kelsen, Hans. „Recognition in International Law, Theoretical Observations“. American Journal of International Law, Vol. 35, Nr. 4 (1941), S. 605-617.

King, Elizabeth M., und Dominique von de Walle. „Catching up slowly: ethnic and gender inequalities in Lao PDR", World Bank - Lao PDR Country Brief No. 2, 1. November 2010.

Kingsbury, Benedict. „,Indigenous Peoples‘ in International Law: A Constructivist Approach to the Asian Controversy". The American Journal of International Law, Vol. 92, Nr. 3 (1. Juli 1998), S. 414-457.

Kingsbury, Benedict, und Kirsty Gover (Hrsg.), Special Issue of the International Journal on Minority and Group Rights: Indigenous Groups and the reviews of Recognition in Asia: Cases from Japan, Taiwan, West Papua, Bali, the People's Republic of China and Gilgit, Vol. 11, No. 1/2 (2004).

Kipuri, Naomi. „The UN Declaration on the Rights of Indigenous Peoples in the African Context". In Making the Declaration Work, the United Nations Declaration on the Rights of Indigenous Peoples, herausgegeben von Claire Charters und Rodolfo Stavenhagen, S. 252-263, 2009.

Klein, Eckart. „Die Frage der Minderheitendefinition“. In Friedenssichernde Aspekte des Minderheitenschutzes in der Ära des Völkerbundes und der Vereinten Nationen in Europa, herausgegeben von Manfred Mohr, 1996.

Klenke, Karin, und Philipp Socha. „Emerging Indigeneity - Völkerrechtswissenschaft und ethnologische Praxis subnationaler kultureller Gemeinschaften“. In Sui Generis Rechte zum Schutz traditioneller kultureller Ausdrucksweisen, Interdisziplinäre Perspektiven, herausgegeben von Kilian Bizer, Matthias Lankau, und Gerald Spindler, Göttinger Studien zu Cultural Property, 2013.

Korn, Horst. „Institutioneller und instrumenteller Rahmen für die Erhaltung der Biodiversität". In 10 Jahre Übereinkommen über die biologische Vielfalt: eine Zwischenbilanz, herausgegeben von Nina Wolff und Wolfgang Köck, 2004.

Kothari, Ashish. Understanding Biodiversity: Life, Sustainability and Equity, 1997.

Krakoff, Sarah, und Kristen A. Carpenter. „Repairing Reparations in the American Indian Nation Context". In Reparations for Indigenous Peoples : International and Comparative Perspectives, herausgegeben von Federico Lenzerini, 2008.

Kugelmann, Dieter. „The Protection of Minorities and Indigenous Peoples Respecting Cultural Diversity“. Max Planck Yearbook of United Nations Law Online, Vol. 11, Nr. 1 (1. Januar 2007), S. 233-263.

Kymlicka, Will. ,Theorizing Indigenous rights“. University of Toronto law journal, Vol. 49, Nr. 2 (1999), S. 281-294.

Lankau, Matthias, und Kilian Bizer. „Die verborgene Effektivität minimaler Resultate in internationalen Verhandlungen: Der Fall der WIPO“. In Die Konstituierung von Cultural Property: Forschungsperspektiven, herausgegeben von Regina Bendix, Kilian Bizer, und Stefan Groth, Göttinger Studien zu Cultural Property, 2010. 
Latif, Ahmed Abdel. „Developing Country Coordination in International Intellectual Property Standard-setting", Trade-related Agenda, Development and Equity (T.R.A.D.E.), Working Papers 24, South Centre, 2005.

Lauterpacht, Hersch. Recognition in international law, 1948.

. „Recognition of States in International Law“. Yale Law Journal, Nr. 53 (1944), S. 385-458.

Lerner, Natan. „The Evolution of Minority Rights in International Law“. In Peoples and Minorities in International Law, herausgegeben von Catherine Brölmann, René Lefeber, und Marjoleine Zieck, 1993.

Group rights and discrimination in international law. International studies in human rights, 1991.

Letschert, Rianne Monique. The impact of minority rights mechanisms, 2005.

Lucas-Schloetter, Agnès. „Folklore“. In Indigenous heritage and intellectual property : genetic resources, traditional knowledge and folklore, herausgegeben von Silke v. Lewinski, 2008.

Ludescher, Monika. Menschenrechte und indigene Völker, 2004.

Machnyikova, Zdenka. „Article 8“. In The rights of minorities in Europe, herausgegeben von Marc Weller, 2006.

Makkonen, Timo. Identity, Difference and Otherness: The Concepts of „People“, Indigenous People' and „Minority " in International Law, 2000.

Malloy, Tove H. „The Title and the Preamble“. In The rights of minorities: a commentary on the European Framework Convention for the Protection of National Minorities, herausgegeben von Marc D. Weller, 2006.

Manby, Bronwen. „South Africa: minority conflict and the legacy of minority rule“. The Fletcher forum of world affairs, Vol. 19, Nr. 1 (1994), S. 27-52.

Mejer, Malwina und Bruno van Pottelsberghe de la Potterie. „Economic Incongruities in the European Patent System". European Journal of Law and Economics, Vol. 34, Nr. 1 (2011), S. 215.

Menon, P. K. The law of recognition in international law, 1994.

Miller, Robert J., Larissa Behrendt, Tracey Lindberg und Jacinta Ruru. Discovering Indigenous Lands: The Doctrine of Discovery in the English Colonies, 2010.

Mohr, Manfred. Friedenssichernde Aspekte des Minderbeitenschutzes in der Ära des Völkerbundes und der Vereinten Nationen in Europa, 1996.

Montes, Adelfo Regino, und Gustavo Torres Cisneros. „The United Nations Declaration on the Rights of Indigenous Peoples: the Foundation of a New Relationship between Indigenous Peoples, States and Societies“. In Making the Declaration Work, the United Nations Declaration on the Rights of Indigenous Peoples, herausgegeben von Claire Charters und Rodolfo Stavenhagen, 2009.

Morse, Bradford W. „Indigenous Peoples of Canada and Their Efforts to Achieve True Reparations“. In Reparations for Indigenous Peoples: International and Comparative Perspectives, herausgegeben von Federico Lenzerini, 2008. 
Murphy, M. Maureen. „The Bureau of Indian Affairs’ Process for Recognizing Groups as Indian Tribes“. CRS Report for Congress, 25. März 2005.

Murray, Rachel. „The UN Declaration on the Rights of Indigenous Peoples in Africa: The Approach of the Regional Organisation to Indigenous Peoples". In Reflections on the UN Declaration on the Rights of Indigenous Peoples, herausgegeben von Steve Allen und Alexandra Xanthaki, 2011.

Murray Li, Tania. „Articulating Indigenous Identity in Indonesia: Resource Politics and the Tribal Slot". Comparative Studies in Society and History, Vol. 42, Nr. 1 (2000), S. 149-179.

Nowak, Manfred. „,The Evolution of Minority Rights in International Law, Comments". In Peoples and Minorities in International Law, herausgegeben von Catherine Brölmann, René Lefeber, und Marjoleine Zieck, 1993.

U.N. covenant on civil and political rights, 2005.

Nußberger, Angelika. „The War between Russia and Georgia - Consequences and Unresolved Questions“. Goettingen Journal of International Law, Russia and International Law - From the North Pole to the Caucasus, Vol. 1, Nr. 2 (2009), S. 341-364.

Packer, John. „Problems in Defining Minorities“. In Minority and group rights in the new millennium, herausgegeben von Deirdre Fottrell, 1999. The protection of ethnic and linguistic minorities in Europe, 1997.

Pernthaler, Peter. „Zur Entstehung des völkerrechtlichen Menschenrechts- und Minderheitenschutzes im 19. und 20. Jahrhundert“". In Handbuch der europäischen Volksgruppen, herausgegeben von Christoph Pan und Beate Sibylle Pfeil, 2000.

Pieterse, Jan Nederveen. „My Paradigm or Yours? Alternative Development, Post-Development, Reflexive Development". Development and change, Vol. 29, Nr. 2 (1998)S. 343-374.

Posey, Darrell A. „Identifizierung und Respektierung der Grenzen zwischen indigenen Völkern, traditionellen Bauern und örtlichen Gemeinschaften“. In Grenzen Los?, herausgegeben von Ernst Ulrich von Weizsäcker, 1997.

Pritchard, Sarah. Der völkerrechtliche Minderheitenschutz: Tübinger Schriften zum internationalen und europäischen Recht, 2001.

Rai, Navin et al., „Implementation of the World Bank's Indigenous Peoples Policy, A Learning Review (FY 2006-2008)“, OPCS Working Paper, August 2011. Zugegriffen 13. Januar 2017. http://siteresources.worldbank.org/INTSAFEPOL/Resources/Indigeno us_peoples_review_august_2011.pdf.

Republic of Bolivia, Ministry of Foreign Affairs and Religion. „Letter to the Director-General of UNESCO“, 24. April 1973, abgedruckt als Annex A: Intergovernmental Copyright Committee, „Proposal for international instrument for the protection of folklore“, 12th session 1973, IGC/XII/12, 1. Oktober 1973. 
Ricketson, Sam, und Jane C. Ginsburg. International copyright and neighbouring rights: the Berne Convention and beyond, 2006.

Riedel, Eibe. „Gruppenrechte und kollektive Aspekte individueller Menschenrechte". In Aktuelle Probleme des Menschenrechtsschutzes. Berichte der Deutschen Gesellschaft für Völkerrecht, 1994.

Robinson, Jacob. „International Protection of Minorities a global view“. Israel Yearbook on Human Rights, Vol. 1, Nr. 1 (1971), S. 61-91.

Roßkopf, Ralf. Theorie des Selbstbestimmungsrechts und Minderheitenrechts. Abhandlungen zu Migration und Flüchtlingsfragen, 2004.

Rosti, Marzia. „Reparations for Indigenous Peoples in Two Selected Latin American Countries". In Reparations for Indigenous Peoples: International and Comparative Perspectives, herausgegeben von Federico Lenzerini, 2008.

Saez, Catherine. „Indigenous Peoples Walk Out Of WIPO Committee On Genetic Resources“. Intellectual Property Watch, 22. Februar 2012. Zugegriffen 14. Januar 2017. http://www.ip-watch.org/2012/02/22/indigenouspeoples-walk-out-of-wipo-committee-on-genetic-resources /.

Saxer, Urs. Die internationale Steuerung der Selbstbestimmung und der Staatsentstehung. Beiträge zum ausländischen öffentlichen Recht und Völkerrecht, 2010.

Scherer-Leydecker, Christian. Minderheiten und sonstige ethnische Gruppen: eine Studie zur kulturellen Identität im Völkerrecht, 1997.

Schillhorn, Kerrin. Kulturelle Rechte indigener Völker und Umweltvälkerrecht - Verhältnis und Vereinbarkeit, 2000.

Schulze, Peter W. „Geopolitics at Work: the Georgian-Russian Conflict“. Goettingen Journal of International Law, Russia and International Law - From the North Pole to the Caucasus, Vol. 1, Nr. 2 (2009), S. 329-340.

Shelton, Dinah. Remedies in international buman rights law, 2005.

Sherkin, Samantha. „A Historical Study on the Preparation of the 1989 Recommendation on the Safeguarding of Traditional Culture and Folklore“. In Safeguarding traditional cultures: a global assessment, herausgegeben von Peter Seitel, 2001.

Sigler, Jay A. Minority rights. Contributions in political science, 1983.

Stamatopoulou, Elsa. „Taking Cultural Rights Seriously: The Vision of the UN Declaration on the Rights of Indigenous Peoples“. In Reflections on the UN Declaration on the Rights of Indigenous Peoples, herausgegeben von Steve Allen und Alexandra Xanthaki, 2011.

Stavenhagen, Rodolfo. „Making the Declaration Work“. In Making the Declaration Work, the United Nations Declaration on the Rights of Indigenous Peoples, herausgegeben von Claire Charters und Rodolfo Stavenhagen, 2009.

Stoll, Peter-Tobias. „Genetische Ressourcen, Zugang und Vorteilsteilhabe“. In 10 Jahre Übereinkommen über die biologische Vielfalt: eine Zwischenbilanæ, herausgegeben von Nina Wolff und Wolfgang Köck, 2004.

Stoll, Peter-Tobias, und Anja von Hahn. „Indigenous peoples, indigenous knowledge and indigenous resources in international law“. In Indigenous 
heritage and intellectual property: genetic resources, traditional knowledge and folklore, herausgegeben von Silke von Lewinski, 2008.

Summers, James. Peoples and international law. Monographs on international law and human rights, 2007.

Taylor, Charles. Multikulturalismus und die Politik der Anerkennung, 2009.

Tennant, Chris. „Indigenous Peoples, International Institutions, and the International Legal Literature from 1945-1993“. In Indigenous Rights, herausgegeben von Anthony J. Connolly, 2009.

Thornberry, Patrick. Indigenous peoples and human rights. Melland Schill studies in international law, 2002.

- „Integrating the UN Declaration on the Rights of Indigenous Peoples into CERD Practice". In Reflections on the UN Declaration on the Rights of Indigenous Peoples, herausgegeben von Steve Allen und Alexandra Xanthaki, 2011.

_. „International and European standards on minority rights“. In Minority rights in Europe: the scope for a transnational regime, herausgegeben von Hugh Miall, 1994.

- International law and the rights of minorities, 1991.

. ,The UN Declaration on the Rights of Persons Belonging to National or Ethnic, Religious and Linguistic Minorities: Background, Analysis, Observations, and an Update“. In Universal Minority Rights, herausgegeben von Alan Phillips und Allan Rosas, 1995.

-. „Who Is Indigenous?" In Economic, Social, and Cultural Rights of the Sami: International and National Aspects, herausgegeben von Frank Horn, 1998.

Thürer, Daniel. Das Selbstbestimmungsrecht der Völker, 1976.

Tomuschat, Christian. „Protection of Minorities under Article 27 of the International Covenant on Civil and Political Rights“. In Völkerrecht als Rechtsordnung, internationale Gerichtsbarkeit, Menschenrechte: Festschrift für Hermann Mosler. Beiträge zum ausländischen öffentlichen Recht und Völkerrecht, 1983.

Trouillot, Michel-Rolph. Global Transformations: Anthropology and the Modern World, 2003.

van der Stoel, Max. OSCE High Commissioner on National Minorities. „Case Studies on National Minority Issues: Positive Results, Keynote Address to the CSCE Human Dimension Seminar, 24 May 1993, Warsaw, Poland“. In Peace and Stability through Human and Minority Rights, herausgegeben von Wolfgang Zellner und Falk Lange, 2001.

Varennes, Fernand de. „Article 10“. In The rights of minorities in Europe, herausgegeben von Marc Weller, 2006.

von Hahn, Anja. Traditionelles Wissen indigener und lokaler Gemeinschaften zwischen geistigen Eigentumsrechten und der ,public domain“. Beiträge zum ausländischen öffentlichen Recht und Völkerrecht, 2004.

Weller, M. The rights of minorities: a commentary on the European Framework. Convention for the Protection of National Minorities, 2006. 
Wenzel, Nicola. Das Spannungsverhältnis zwischen Gruppenschutz und Individualschutz im Völkerrecht, 2008.

Wiessner, Siegfried. United Nations Declaration on the Rights of Indigenous Peoples, General Assembly resolution 61/295, Audiovisual Library of the United Nations, 2009.

Wintgens, Hugo. Der völkerrechtliche Schutz der nationalen, sprachlichen und religiösen Minderheiten, 1930.

Wolfrum, Rüdiger. „Völkerrechtlicher Rahmen für die Erhaltung der Biodiversität". In 10 Jahre Übereinkommen über die biologische Vielfalt: eine Zwischenbilan₹, herausgegeben von Nina Wolff und Wolfgang Köck, 2004.

Xanthaki, Alexandra. Indigenous Rights and United Nations Standards, 2007. 
$M$ inderheiten, indigene Völker und lokale Gemeinschaften haben in der Völkerrechtsordnung einen eigenen Status, da ihre Position in der Politik der Mehrheitsgesellschaft eines besonderen Schutzes bedarf und innen als Gruppe bestimmte Rechte zustehen. Ungeklärt ist dabei jedoch: Wie lassen sich diese kulturell distinkten substaatlichen Gruppen rechtlich bestimmen? Wem stehen die völkerrechtlichen Garantien des Minderheitenschutzes, das Recht auf Selbstbestimmung oder die Rechte an traditionellem Wissen zu? Erfolglos drehen sich die politisch geprägten Debatten in Wissenschaft und Praxis um Definitionsansätze und Kriterien kultureller Unterschiedlichkeit.

Die vorliegende Arbeit zeigt, dass es die Anerkennung als ,Minderheit', ,indigenes Volk' oder ,lokale Gemeinschaft' ist, die den konstitutiven Akt für den Status einer Gruppe bildet. Im Gegensatz zu den politischen Definitionsdebatten ermöglicht es die hier dargestellte Perspektive der Anerkennung, die statusrelevanten Prozesse und Institutionen in den Blick zu nehmen. Die Anerkennung substaatlicher Gruppen ist in Anlehnung an die Staatenanerkennung völkerrechtlich zu verorten und lässt sich interdisziplinär verankern. Die Perspektive der Anerkennung löst die Diskussion um allgemeine kriteriale Definitionen ab und ermöglicht die Machtungleichgewichte und Interessenkonflikte zu erkennen, die der Statusfrage von kulturell distinkten substaatlichen Gruppen inhärent sind. 